\title{
Mapping individual trees from airborne multi-sensor imagery
}

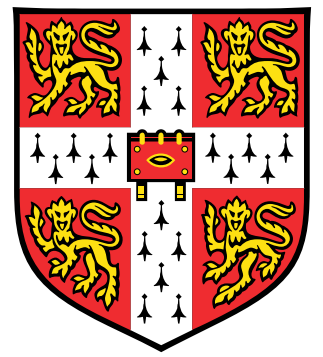

Juheon Lee

Department of Plant Sciences

University of Cambridge

This dissertation is submitted for the degree of

Doctor of Philosophy

Fitzwilliam College

March 2016 

I would like to dedicate this thesis to my sister and my parents ... 



\section{Declaration}

I hereby declare that except where specific reference is made to the work of others, the contents of this dissertation are original and have not been submitted in whole or in part for consideration for any other degree or qualification in this, or any other university. This dissertation is my own work and contains nothing which is the outcome of work done in collaboration with others, except as specified in the text and Acknowledgements. This dissertation contains fewer than 65,000 words including appendices, bibliography, footnotes, tables and equations and has fewer than 150 figures.

Juheon Lee

March 2016 



\section{Acknowledgements}

First and foremost, I would like to thank my supervisors Dr David Coomes and Dr CarolaBibiane Schönlieb for teaching me the beauty of mathematics and its application to ecology. David, you have been a tremendous mentor for me in every way. I will never forget the beautiful memories during my PhD study, like the Spanish fieldwork when you got lymes disease! We should organise another trip to Spain to finish our work. Carola, you have taught me how beautiful mathematics is. Without you, I might have taken different road entirely. I appreciate your advice and help. I am going to miss your great party at 37 New Square!

Many thanks to Dr Xiaohao Cai who helped me with image processing and mathematics in general, Prof Jan Lellmann, who taught and advised me about convex optimisation and convergence analysis, and Dr Michele Dalponte for a fantastic collaboration on tree segmentation. I also have to thank Dr Orsola Rath Spivack and Dr Mark Spivack for inspiring me about developing a tree segmentation algorithm.

I thank to all members of the Forest Ecology and Conservation group and the Image Analysis group, who provide me with both intellectual and moral support. In alphabetical order: Marie D’Autume, Martin Benning, Luca Calatroni, Antonin Chambolle, Veronica Corona, Jessica Da Silva, Joana Grah, Maria Hänel, Rob Hocking, Tommaso Jucker, Rafi Kent, Gillian Lui, William Lynam, Matheus Nunes, Kostas Papafitsoros, Evangelos Papoutsellis, Simone Parisotto, Andres Plaza, Will Simonson, Minerva Singh, Rebecca Spriggs, Yoeri Boink, Tuomo Valkonen, Beatrice Wedeux, Gaohang Yu, Ping Zhong and anyone who 
I may have unintentionally forgotten. Very special thanks to Kee-hoon Kang, Jamie-Oliver Males, Tommaso Jucker and Boris Bongalov for commenting on my thesis!

Last but not least, I owe a debt of gratitude (also money) to my family. I especially thank to my sister - Kyung-rin Lee - for supporting my 5 years stay in England. You know how much I love you! I am so glad that we can live together in South Korea again (at least for a while)! I thank my parents for supporting me throughout my life in general. Your prayer for me was what sustained me thus far. And finally, I am grateful to my lovely cat - dotori (acorn in English) - I could not have managed to finish without your support. 


\begin{abstract}
Airborne multi-sensor imaging is increasingly used to examine vegetation properties. The advantage of using multiple types of sensor is that each detects a different feature of the vegetation, so that collectively they provide a detailed understanding of the ecological pattern. Specifically, Light Detection And Ranging (LiDAR) devices produce detailed point clouds of where laser pulses have been backscattered from surfaces, giving information on vegetation structure; hyperspectral sensors measure reflectances within narrow wavebands, providing spectrally detailed information about the optical properties of targets; while aerial photographs provide high spatial-resolution imagery so that they can provide more feature details which cannot be identified from hyperspectral or LiDAR intensity images. Using a combination of these sensors, effective techniques can be developed for mapping species and inferring leaf physiological processes at ITC-level.

Although multi-sensor approaches have revolutionised ecological research, their application in mapping individual tree crowns is limited by two major technical issues: (a) Multi-sensor imaging requires all images taken from different sensors to be co-aligned, but different sensor characteristics result in scale, rotation or translation mismatches between the images, making correction a pre-requisite of individual tree crown mapping; (b) reconstructing individual tree crowns from unstructured raw data space requires an accurate tree delineation algorithm. This thesis develops a schematic way to resolve these technical issues using the-state-of-the-art computer vision algorithms. A variational method, called NGF-Curv, was developed to co-align hyperspectral imagery, LiDAR and aerial photographs.
\end{abstract}


NGF-Curv algorithm can deal with very complex topographic and lens distortions efficiently, thus improving the accuracy of co-alignment compared to established image registration methods for airborne data. A graph cut method, named MCNCP-RNC was developed to reconstruct individual tree crowns from fully integrated multi-sensor imagery. MCNCP-RNC is not influenced by interpolation artefacts because it detects trees in $3 \mathrm{D}$, and it detects individual tree crowns using both hyperspectral imagery and LiDAR.

Based on these algorithms, we developed a new workflow to detect species at pixel and ITC levels in a temperate deciduous forest in the UK. In addition, we modified the workflow to monitor physiological responses of two oak species with respect to environmental gradients in a Mediterranean woodland in Spain. The results show that our scheme can detect individual tree crowns, find species and monitor physiological responses of canopy leaves. 


\section{Table of contents}

$\begin{array}{ll}\text { List of figures } & \text { xv }\end{array}$

$\begin{array}{ll}\text { List of tables } & \text { xxiii }\end{array}$

$\begin{array}{ll}\text { Nomenclature } & \text { xxvii }\end{array}$

1 Introduction 1

1.1 General Introduction $\ldots \ldots \ldots \ldots 1$

1.2 Forest structures using LiDAR . . . . . . . . . . . . . . . . . 4

1.3 Hyperspectral imagery and spectranomics . . . . . . . . . . . 8

1.4 Ecological applications of LiDAR and hyperspectral imagery fusion . . . . 11

1.5 Technical difficulties of hyperspectral and LiDAR fusion . . . . . . . . . . 14

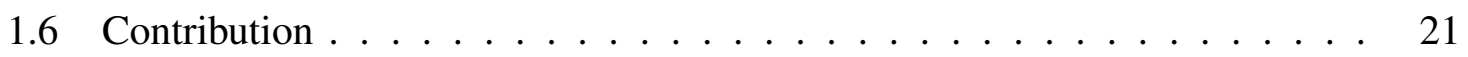

1.6.1 Non-parametric image registration of airborne LiDAR, hyperspectral and photographic imagery of wooded landscapes . . . . . . . 21

1.6.2 3D individual tree Segmentation of integrated airborne LiDAR and optical imagery using normalised cut with priors . . . . . . . 22

1.6.3 Individual tree species classification from airborne multi-sensor im-

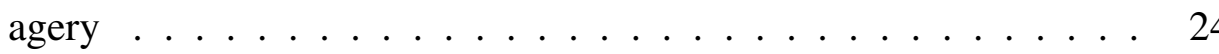

1.6.4 Physiological indices of individual trees in Mediterranean woodland estimated using fused airborne LiDAR and hyperspectral imagery . 
1.6.5 Organisation of the thesis . . . . . . . . . . . 28

2 Non-parametric image registration of airborne LiDAR, hyperspectral and photographic imagery of wooded landscapes

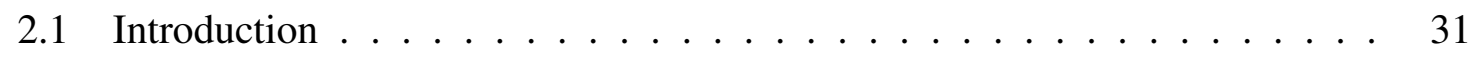

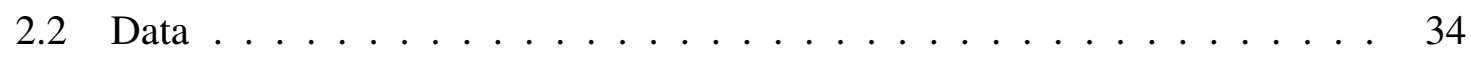

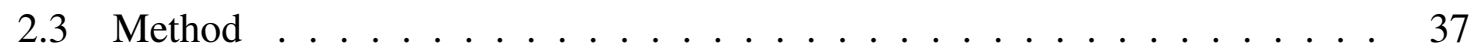

2.4 Application of the registration approach to the airborne remote sensing dataset 41

2.5 Results............................... 44

2.6 Concluding remarks . . . . . . . . . . . . . . . . . . . 51

3 3D individual tree segmentation of integrated airborne LiDAR and optical imagery using normalised cut with priors

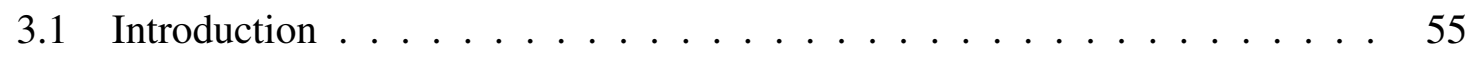

3.2 The theoretical background of normalised cut framework . . . . . . . . 58

3.3 Methods ........................... 61

3.3.1 Registration of remote sensing datasets . . . . . . . . . . 62

3.3 .2 Feature extraction . . . . . . . . . . . . 63

3.3.3 Local maxima detection . . . . . . . . . . . . . . . . 64

3.3.4 MultiClass Normalised Cut with Priors (MCNCP) . . . . . . . . . 64

3.4 Datasets description and design of experiments $\ldots \ldots \ldots$

3.5 Results . . . . . . . . . . . . . . . . . . . 69

3.5.1 Tree delineation using LiDAR imagery . . . . . . . . . . . . 69

3.5.2 Tree delineation from LiDAR and hyperspectral imagery . . . . . . 75

3.6 Discussion . . . . . . . . . . . . . . . . . . 77

3.6.1 The application of graph cut approaches to tree delineation . . . . 77

3.6.2 Combining LiDAR and hyperspectral imagery to improve delineation 78 
3.6.3 The problem of detecting understory trees . . . . . . . . 80

3.6.4 Concluding remarks . . . . . . . . . . . . . 82

4 Individual tree species classification from airborne multi-sensor imagery $\quad 83$

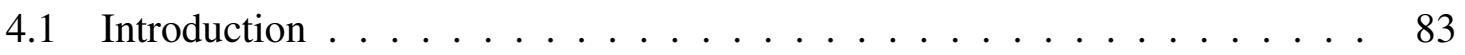

4.2 Data description . . . . . . . . . . . . . . . 87

4.2.1 Study site and field data $\ldots \ldots \ldots$. . . . . . . . . 87

4.2.2 Airborne survey . . . . . . . . . . . . . . . . . 87

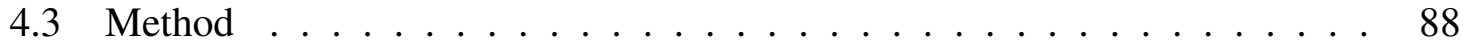

4.3.1 Co-alignment of LiDAR and hyperspectral imagery . . . . . . . . 89

$4.3 .2 \quad$ Feature extraction . . . . . . . . . . . . . . . . 89

4.3.3 Individual tree crown delineation _ . . . . . . . . . . . . . . 89

4.3.4 Species classification . . . . . . . . . . . . . . . 9 90

4.4 Results and discussion $\ldots \ldots \ldots$. . . . . . . . . . . . . . . 92

4.4.1 Pixel-level tree species classification . . . . . . . . . . . . . . 94

4.4.2 ITC-level species classification - mapping individual trees . . . . . 98

4.5 Concluding remarks . . . . . . . . . . . . . . . . . . . . 102

5 Physiological indices of individual trees in Mediterranean woodland estimated $\begin{array}{lr}\text { using fused airborne LiDAR and hyperspectral imagery } & 103\end{array}$

5.1 Introduction . . . . . . . . . . . . . . . . . . 103

5.2 Data description and design of experiments . . . . . . . . . . . 105

$5.2 .1 \quad$ Study area . . . . . . . . . . . . . . . . 105

5.2.2 Airborne image collection and pre-processing . . . . . . . . . 106

5.2.3 Multi-sensor data processing and analysis . . . . . . . . . . . 109

5.3 Result ......................... 116

5.4 Discussion . . . . . . . . . . . . . . . . . . . 120 
5.4.1 Photosynthetic light use efficiency . . . . . . . . . . . . . . . 120

5.4 .2 Canopy water contents . . . . . . . . . . . . . . 121

5.4.3 Carotenoids and anthocyanins variations . . . . . . . . . . 122

5.4 .4 Limitations and future work . . . . . . . . . . . . 123

5.5 Concluding remarks . . . . . . . . . . . . . . . . . . 126

$\begin{array}{lll}6 & \text { General discussion } & 127\end{array}$

6.1 Nonparametric image registration of airborne LiDAR, hyperspectral and photographic imagery of wooded landscapes . . . . . . . . . . . 128

6.1 .1 Limitation . . . . . . . . . . . . . . . . . . . . . 129

6.1 .2 Future work . . . . . . . . . . . . . . . . 130

6.2 3D individual tree segmentation of integrated airborne LiDAR and optical imagery using normalised cut with priors . . . . . . . . . . . . 131

6.2 .1 Limitation . . . . . . . . . . . . . . . . . . . 132

6.2 .2 Future work . . . . . . . . . . . . . . . 133

6.3 Individual tree species classification from airborne multi-sensor imagery . . 134

6.3 .1 Limitation . . . . . . . . . . . . . . . . . 135

6.3 .2 Future work . . . . . . . . . . . . . 136

6.4 Physiological indices of individual trees in Mediterranean woodland estimated using fused airborne LiDAR and hyperspectral imagery $\ldots$. . . . 137

6.4 .1 Limitation . . . . . . . . . . . . . . . . . . 137

6.4 .2 Future work . . . . . . . . . . . . . . . . 138

6.5 Concluding remark . . . . . . . . . . . . . . . . . 139

$\begin{array}{ll}\text { References } & 141\end{array}$ 


\section{List of figures}

1.1 An example of airborne LiDAR point cloud (a) and a pictorial illustration of the reconstruction of forest structures (b) (provided by Dr. Simonson). . . . 9

1.2 The basic concept of hyperspectral data . . . . . . . . . . . . . . . . 11

1.3 The impacts of 2010 drought on Amazonian forests. The red colour indicates stressed trees (top image), whereas white indicates leafless branches (bottom image). .............................. 14

1.4 The absolute difference between a registered hyperspectral image $(T)$ and a LiDAR intensity image $(R)$. (i.e. $|T-R|$ ) $\ldots \ldots \ldots 22$

1.5 An example of MCNCP-RNC segmentation. (a) indicates a LiDAR point cloud and (b) shows ITCs in different colours. . . . . . . . . . . . . . . . . 24

1.6 Mapping individual tree species at ITC-level and ground truth. The background images in (a)-(c) are the digital surface model derived from LiDAR data. The coloured map in (c) is pixel-level species classification, where each colour indicates different species. The circles in different colours represents ITCs of different species. More details will be explained in Chapter 4 . . . 26 
1.7 Variation in PRI obtained from the hyperspectral imagery of (a) $59962 Q$. suber and (b) 31784 Q. canariensis trees with respect to altitude. Black line indicates the curve fitted by regression. Colour represent density of observations scaled from blue to red, i.e. red indicates high frequency of observations, and blue means low density of observations fitted using the dscatter function in MATLAB . . . . . . . . . . . . . 28

2.1 The aerial coverages of LiDAR (blue line), hyperspectral images (green line) and aerial photographs (red line) recorded in line 2 of the NERCARSF survey of Spanish woodland. The LiDAR aerial coverage is about 6.4 $\mathrm{km}^{2}$ with $1 \mathrm{~m}$ spatial resolution, while the hyperspectral imagery and aerial photographs are $28.4 \mathrm{~km}^{2}$ and $23.4 \mathrm{~km}^{2}$ respectively. The spatial resolutions of hyperspectral imagery and aerial photos are $3 \mathrm{~m}$ and $0.3 \mathrm{~m} . \ldots 36$

2.2 Image registration of a hyperspectral image onto a LiDAR intensity image of a Spanish woodland, surveyed by aircraft $\left(\right.$ scale $\left.400 \times 400 \mathrm{~m}^{2}\right)$. The first row shows (A1) a LiDAR intensity reference image $R$; (A2) a hyperspectral template image $T$; (A3) original colour image of (A2); (B0) a map showing the difference between $T$ and $R$ (i.e. $|T-R|$ ), which would be entirely dark if the match was perfect; $(\mathrm{C} 0)$ highlights the circled area of (B0). The second row of maps show $\left|T_{\text {reg }}-R\right|$ obtained by using established methods NCC, MI, NGF, SURF as well as our NGF-Curv method. The results of registration methods are denoted by $T_{r e g}^{\mathrm{NCC}}, T_{r e g}^{\mathrm{MI}}, T_{r e g}^{\mathrm{NGF}}, T_{r e g}^{\mathrm{SURF}}$ and $T_{\text {reg }}^{\mathrm{NGF}-\mathrm{Curv}}$, respectively, in these panels; yellow circle highlights areas of the images where differences among registration methods are seen. The third row zooms in on these highlighted circles. The final row of panels shows the aerial photograph template image after it has been registered using methods (D1) NCC, (D2) MI, (D3) NGF, (D4) SURF and (D5) NGF-Curv. . . . . . 46 
2.3 Image registration of an aerial photograph onto a hyperspectral image in a region which has flat terrain (scale $2400 \times 1800 \mathrm{~m}^{2}$ ). The first row shows (A1) a hyperspectral reference image $R$; (A2) an aerial photograph template image $T$; (B0) a map showing difference between these images (i.e. $|T-R|$ ); (C0) highlight the circle region of (B0). The second row shows maps of $\left|T_{\text {reg }}-R\right|$ generated by methods NCC, MI, NGF, SURF and our NGF-Curv approach. The third row highlights the circle areas of the maps in the second row. The last row of panels shows the aerial photograph template image after it has been registered using methods (D1) NCC, (D2) MI, (D3) NGF, (D4) SURF and (D5) NGF-Curv.

2.4 Image registration of an aerial photograph onto a hyperspectral image in the case of rugged terrain (scale $2400 \times 1800 \mathrm{~m}^{2}$ ). The first row shows (A1) a hyperspectral reference image $R$; (A2) an aerial photograph template image $T$; the second row shows (B0) a map of difference between these images (i.e. $|T-R|$ ) for methods NCC, MI, NGF, SURF and NGF-Curv. The third row $(\mathrm{C} 0)$ zooms in on the circular regions of (B0). The last row of panels shows the aerial photograph template image after it has been registered using methods (D1) NCC, (D2) MI, (D3) NGF, (D4) SURF and (D5) NGF-Curv.

2.5 Checkerboard overlay between aerial photograph template images $T$ and hyperspectral reference images $R$ and checkerboard overlay of registered aerial photograph template images $\left(T_{\text {reg }}^{\mathrm{NGF}-\mathrm{Curv}}\right)$ and hyperspectral reference images $(R)$ generated by our NGF-Curv approach. (A)-(B) Flat terrain case; (C)-(D) rugged terrain case. . . . . . . . . . . . . . 
2.6 Fully registered LiDAR, hyperspectral and aerial photograph imagery. (A) LiDAR intensity image; (B) RGB bands of hyperspectral imagery; (C) mosaic imagery of registered aerial photographs of our NGF-Curv approach with fixed global regularisation parameter $\alpha$; (D) mosaic imagery of registered aerial photographs of the NGF-Curv approach with locally tuned regularisation parameter $\alpha$; (E1)-(E4) highlights of the circle regions in

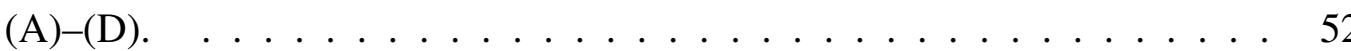

3.1 The workflow of individual tree segmentation using LiDAR only (solid line), and LiDAR with hyperspectral imagery (dashed line). . . . . . . . . . . . . 62

3.2 Examples of MCNCP-RNC tree delineation for a plot in the Italian dataset (plot 77). Results are projected onto a 2D plane: each delineated tree is shown in red circles, and the locations of the ground truth are shown in blue pentagons. The dark circle (solid line) shows the $15 \mathrm{~m}$ radii field plot, where ground truth were measured. The dark solid line shows matching of segmented trees and ground truth. The numbers in red and blue colours indicate tree heights of segmented trees and ground truth, respectively. . . .

3.3 3D examples of individual tree delineation by RNC (left) and MCNCP-RNC (right) algorithms in the Italian dataset (plot 77). . . . . . . . . . . . 70

3.4 Examples of MCNCP-RNC segmentation of the NEWFOR benchmark datasets. The first row shows the LiDAR point clouds from test sites 7 (Left) and 16 (Right). The second row presents the results of our ITC delineation method

3.5 Examples of hyperspectral images in the Italian and English datasets. The blue circle and square represent the size of test sites of the Italian $\left(706 \mathrm{~m}^{2}\right)$ and English datasets (18 ha), respectively. . . . . . . . . . . . 
3.6 Example of MCNCP-RNC segmentation of understory tree in the Italian dataset. The black solid line is the interpolation line (CHM) of the LiDAR point cloud. Point clouds in sienna and purple colours are the segmented ITCs using MCNCP-RNC . . . . . . . . . . . . . . . . . 80

4.1 Workflow used to detect individual tree crown and identify their species by fusing LiDAR and hyperspectral imagery. . . . . . . . . . . . . . 88

4.2 Training and test samples for species classification in the English dataset. The coloured polygons in (a) and (b) represent training and test samples of each species overlaid over a false colour representation of the hyperspectral imagery. The colour map (c) shows the result of the pixel-level species classification by our proposed workflow. Different colours imply different species, i.e., blue colour represents Larix decidua, green colour represents Acer pseudoplatanus, red colour represents Fraxinus excelsior, yellow colour represents Fagus sylvatica, purple colour represents Quercus robur, brown colour represents Betula spp., and white pixels indicate shaded pixels . . .

4.3 The results of the images co-alignment, feature reduction and ITC delineation in our workflow in the English dataset. The first row shows the image registration between LiDAR DSM (a) and RGB true colour hyperspectral imagery (b) and the co-aligned hyperspectral imagery in RGB true colour (c). The second row shows the first three principal components (d)-(f). The third row shows examples of the MCNCP-RNC segmentation viewed obliquely (g)-(i) and different colours represent ITCs. . . . . . . . . . . . . . . 93 
4.4 Mapping individual tree species at ITC-level and ground truth over the 18ha English dataset. The background images in (a)-(c) are DSM. The coloured map in (c) is pixel-level species classification, where each colour indicates different species. The circles in different colours represents ITCs of different species. Blue colour represents Larix decidua, green colour represents Acer pseudoplatanus, red colour represents Fraxinus excelsior, yellow colour represents Fagus sylvatica, purple colour represents Quercus robur, and brown colour represents Betula spp. . . . . . . . . . . . . . . 999

4.5 Mapping each species at ITC-level and the ground truth. Each circle represents a single ITC. The first row shows the ground truths canopy trees over $18 \mathrm{~m}$. The second row shows the results of ITC-level species classification. Different colours imply different species, i.e., red colour represents Fraxinus excelsior, green colour represents Acer pseudoplatanus, purple colour represents Quercus robur, blue colour represents Larix decidua, yellow colour represents Fagus sylvatica, and brown colour represents Betula spp. . . . . 101

5.1 Location of Los Alcornocales, Southern Spain (provided by Dr. Simonson). The red rectangular box indicate the places where hyperspectral and LiDAR data were obtained. . . . . . . . . . . . . . . . . 106

5.2 The RGB true colour composite (460, 550 and 640nm) of hyperspectral

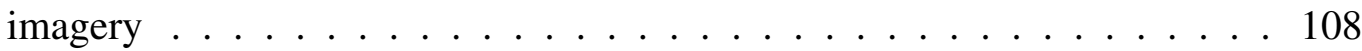

5.3 The workflow for analysing spectral indices at ITC level . . . . . . . . . . 109

5.4 Results of tree delineation: (a) scatter plots of ITCs, and (b) 3D shapes of ITCs generated by convex hull. . . . . . . . . . . . . . . . . 111 
5.5 Examples of two oak species distribution at valley bottom. Each panel shows the results of species classification (a), RGB composites of hyperspectral imagery (b) and elevation map (c), respectively. $Q$. canariensis and $Q$. suber are shown in red and green colours in (a). The dark colour in (a) represents filtered pixels, which are either ill-conditioned or non-vegetation. The blue circle indicates the location of valley bottom. . . . . . . . . . . . . 112

5.6 Species classification results of hyperspectral data. Q. canariensis and $Q$. suber are shown in red and green colours, respectively. . . . . . . . . . . 113

5.7 Variation in (a) PRI, (b) CRI, (c) WBI (d) ARI (e) RGRI obtained from the hyperspectral imagery of 59962 Q. suber trees with respect to altitude. Blue line indicates fitting curve. . . . . . . . . . . . . . . . 117

5.8 Variation in (a) PRI, (b) CRI, (c) WBI (d) ARI (e) RGRI obtained from the hyperspectral imagery of 31784 Q. canariensis trees with respect to altitude. Black line indicates fitting curve. . . . . . . . . . . . . . . . . 118 



\section{List of tables}

1.1 The list of algorithms that co-align multi-sensor imagery . . . . . . . . 15

1.2 The list of methods that delineate individual trees from LiDAR. . . . . . . . 17

1.3 The list of methods that classify and map tree species from either hyperspectral imagery or hyperspectral imagery alongside LiDAR. . . . . . . . . . . 19

2.1 Mean, standard deviation, minimum and maximum of $\left|T_{\text {reg }}-R\right|$, correlation between $T_{\text {reg }}$ and $R$, and CPU time in seconds of each method . . . . . . . 45

3.1 The parameter settings for the experiments . . . . . . . . . . . . . . 69

3.2 The performance of delineation algorithms when applied to seven forest plots in the Italian datasets. Inside the bracket the method used to obtain local maxima is shown. 'Ground Truth' is the number of stems $(>1 \mathrm{~cm}$ DBH) recorded in the field plots. 'Extracted' means the number of trees delineated by the algorithms. while 'matched' indicates the number of correctly segmented trees, assessed by the NewFor matching algorithm. .

3.3 The summary of the performance of delineation algorithms in the Italian dataset at different height scales. 'Extract' means the number of trees delineated by the algorithms. 'Match' is the number of trees that were matched to trees in seven mapped forest plots which had similar $(\mathrm{x}, \mathrm{y})$ coordinates and were of similar heights. . . . . . . . . . . . . . . 
3.4 The NewFor benchmark test of TIFFS and MCNCP-RNC. 'Extract' means the number of trees delineated by the algorithms. 'Match' defines the number of correctly assigned trees. . . . . . . . . . . . . . . 73

3.5 The summary of the performance of delineation algorithms in the NewFor benchmark dataset in different tree height tiers. 'Extract' means the number of trees delineated by the algorithms. 'Match' is the number of trees that were matched to trees in the mapped forest plot which had similar $(\mathrm{x}, \mathrm{y})$ coordinates and were of similar heights $\ldots \ldots \ldots 73$

3.6 The performance of the delineation algorithms in the English dataset, by height tier. 'Extract' means the number of delineated trees. 'Match' is the number of trees that were matched to trees in the mapped forest plot which had similar $(\mathrm{x}, \mathrm{y})$ coordinates and similar heights. In the first column, the range of heights in each tier is shown. . . . . . . . . . . . . 75

3.7 Computation time in seconds of RNC, MCNC, TIFFS and MCNCP-RNC applied to the Italian dataset. . . . . . . . . . . . . . . . 76

4.1 The numbers of individual and canopy trees of six species recorded in the English dataset . . . . . . . . . . . . . . . 87

4.2 Number of pixels for each species of the training and test samples for tree species classification . . . . . . . . . . . . . . . . 90

4.3 Species classification results from the ITSC method . . . . . . . . . . . . 96

4.4 Species classification results obtained by the ITSC-R method . . . . . . . . 96

4.5 ITC delineation accuracy with commission and omission errors . . . . . . . 101

4.6 Species classification results at ITC-level. . . . . . . . . . . . . . . . . . 102

5.1 Spectral indices for estimating biophysical and chemical properties of vegetation. $\mathrm{R}$ is reflectance and subscripts give the wavelengths in $\mathrm{nm} \quad \ldots . .114$ 
5.2 Regression models between chemical indices and elevation. $x$ is altitude and $\beta_{i}(i=1,2,3)$ is regression coefficients $\ldots \ldots \ldots . \ldots \ldots$ 



\title{
Nomenclature
}

\author{
Acronyms / Abbreviations
}

AGC Automatic Gain System

ARI Anthocyanin Reflectance Index

ARS Airborne Remote Sensing

AVHRR Advanced Very High Resolution Radiometer

BRDF Bi-directional Reflectance Distribution Function

CHM Canopy Height Model

CRI Carotenoid Reflectance Index

DBH Diameter at Breast Height

DSM Digital Surface Model

FAIR Fast Image Registration software

GCP Ground Control Point

GLAS Geoscience Laser Altimeter System

ICESat Ice, Cloud and land Elevation Satellite 
ITC Individual Tree Crown

ITSC Individual Tree Species Classification

ITSC-R Individual Tree Species Classification with Robust principal component analysis

LAI Leaf Area Index

Landsat Land satellite

libSVM A library for Support Vector Machine

LiDAR Light Detection and Ranging

LUE Light Use Efficiency

MCNCP Multi-Class Noramlised Cut with Priors

MCNCP-RNC Multi-Class Noramlised Cut with Priors - Recursive Normalised Cut

MI Mutual Information

MODIS Moderate Resolution Imaging Spectroradiometer

MWF Moving Window Filtering

NCC Normalised Cross Correlation

NC Normalised Cut

NDVI Normalised Difference Vegetation Index

NERC-ARSF The Airborne Research and Survey Facility of the UK's Natural Environment Research Council

NGF-Curv Normalised Gradient Field with Curvature regularisation method 
NGF Normalised Gradient Field

NN Nearest Neighbour

NPP Net Primary Production

OSGB Ordinance Suvey Great Britain

PCA Principal Component Analysis

PRI Photochemical Reflectance Index

RBF Radical Basis Function

RGB Red, Green and Blue colour model

RGRI Red Green Ratio Index

rPCA robust PCA

SAR Synthetic Aperture Radar

SIFT Scale-Invariant Feature Transform

STRM Shuttle Radar Topography Mission

SURF Speeded-Up Robust Feature

SVM Support Vector Machine

TIFFS Toolbox for LiDAR data Filtering and Forest Studies

UNFCCC United Nations Framework Convention on Climate Change

UTM Universal Transverse Mercator projection

WBI Water Band Index

WGS-84 World Geodetic System - 1984 



\section{Chapter 1}

\section{Introduction}

\subsection{General Introduction}

How nutrients and energy circulate through forest ecosystems [73] is a question that has received significant attention in ecology, particularly in the context of anthropogenic global change that alters disturbance regimes, forest dynamics and biodiversity patterns $[174,214,210,214,249]$. In particular, estimating forest carbon stocks, and how they are changing with time, have been the focus of much international attention, because forest carbon is an important component of the global carbon cycle [46, 212]. Anthropogenic carbon emissions from fossil fuel consumption and large-scale land use change are triggering changes in the world's climate systems, sparking efforts to reduce emissions through international agreements including the United Nations Framework Convention on Climate Change (UNFCCC) and the Kyoto Protocol [117, 183, 212, 158, 184, 190]. Countries that ratified these agreements are obliged to report on their effort to reduce direct carbon emission arising from fossil fuel consumption and indirect emissions arising from land use change and forest disturbance $[183,212,190]$. Although knowledge about the effects of forest perturbation and land-use change on carbon storage is growing, considerable uncertainty remains $[211,31]$. As the forest ecosystems are heterogeneous, mapping and estimating carbon storage on a 
large scale is very challenging [211], and tracking change using conventional field surveys is labour-intensive and time-consuming, relying on sampling from small and spatially dispersed plots. Field plot measurements of carbon stocks use allometric models that are speciesspecific or very accurate species groups, thus making them difficult to extrapolate to national or global scales [144, 211].

Remote sensing technologies provide an increasingly practical approach to mapping and monitoring forest dynamics at large scales [17] [18]. Remote sensing techniques can be categorised as active or passive. Active sensing involves emitting a signal to the ground, then measuring the intensities of the returning signal. For example, synthetic aperture radar (SAR) sensors emit radio wave frequency radiation towards the ground, then record the returning echos. Light detection and Ranging (LiDAR) transmits a monochromatic pulse of energy towards the ground, then measures the intensity of the returning wave of energy over time, which encodes the 3D structure of the reflective surface. Passive sensing, on the other hand, only measures the natural reflectance of a target. Landsat 8 , for instance, measures the ground reflectance within bands spread over from the visible to thermal infrared region [2].

Both active and passive sensors have been used to estimate biomass, forest carbon and land cover changes from space[261]. Active sensors such as the Geoscience Laser Altimeter System (GLAS) mounted on the Ice, Cloud and land Elevation Satellite (ICESat) [175] and SAR $[165,208,195,196]$ have been used to estimate forest canopy height and biomass. In addition, many passive sensors such as Moderate Resolution Imaging Spectroradiometer (MODIS), LandSAT, and the advanced very high resolution radiometer (AVHRR) [45] have been used to estimate forest productivity [94, 261, 45, 64]. Wessels et al. [261] used AVHRR time series to estimate the variation of terrestrial net primary production (NPP), which is the time integral of the positive increment to plant biomass [94]. Brown et al. [45] evaluated LandSat, MODIS and AVHRR sensors to calibrate the relationship between net primary production and spectral index, i.e. the ratio between two or many different spectral bands 
[64]. These satellite platforms have advanced understanding of the link forest dynamics and anthropogenic climate change [238]. However, mapping forest biomass in detail is still challenging from space, because the resolution of satellite images is coarse in the case of optical imagery, while the ICESat LiDAR mission has sampled the earth's surface rather than providing wall-to-wall information.

Airborne remote sensing bridges the gap between global-scale biomass estimation by satellites and ground-based measurements provided by forest inventories. Airborne remote sensing can allow the examination of the biomass and species identity of individual tree crowns (ITCs) with direct measurement $[18,93,189]$. LiDAR provides very accurate canopy height, volume and structural information when flown on a plane, while passive sensors give spectral information in visible to thermal bands, thus providing both biophysical and chemical properties of canopy foliage [213, 15, 18, 232]. Several groups are developing methods to estimate the chemical and physical traits of foliage from aircraft, perhaps the best known of which is Asner's group at the Carnegie institute [15, 18, 17], which has advanced "spectranomic" approaches considerably $[18,16]$.

The overall objective of my thesis is to improve upon the image processing techniques currently used to process airborne remote sensing (ARS) data. Despite rapid advances, ARS data are seldom used to map individual trees because of the technological challenges involved with fusing datasets precisely, recognising individual tree crowns and extracting meaningful information from the hyperspectral images of individual crown. Although my interests are primarily in image analysis, my thesis is interdisciplinary in nature, combining those interests with remote sensing and ecology. So this introductory chapter attempts to provide a short overview of recent developments in remote sensing and ecology, setting the context for my four chapters dealing with different aspects of image analysis.

This introduction briefly introduces the application of airborne remote sensing technology to ecological research. I first describe LiDAR and how it is already being used to map 
Introduction

the biomass of individuals, as well as provide regional and global maps of carbon stocks and fluxes. Then, I describe state-of-the-art hyperspectral imaging techniques and their application in biogeosciences. Then, I provide an explanation of how researchers are fusing LiDAR and hyperspectral imagery, and outline some applications of these combined datasets. Finally, I set out some of the technical difficulties of fusion of LiDAR and hyperspectral imagery and list the main aims of the thesis.

\subsection{Forest structures using LiDAR}

LiDAR is a type of active remote sensing technique. LiDAR emits pulses of monochromatic light (typically $1064 \mathrm{~nm}$ in most commercial LiDAR sensors for aircraft) in the direction of a surface, then measures the returning signals in discrete or full-waveform. The earliest published work on using airborne LiDAR to estimate forest dynamics and biomass dates back to 1960 s $[225,202]$. With the advances of navigation system in 1990 s, commercial airborne LiDAR has been widely available for ecological research $[172,174,189]$. On the other hand, one of the first satellite LiDAR system, called shuttle laser altimeter, was launched in January, 1996 [106]. ICESat/GLAS launched in 2003 have successfully demonstrated the capability of monitoring forest biomass using a satellite LiDAR platform, although its primary objective was monitoring ice sheets mass balance $[1,175]$. These airborne and satellite LiDAR systems have different characteristics, each with their own strengths and weaknesses. This chapter will review how the LiDAR system works at individual-tree, regional and global scales for forest biomass estimation and ecological research.

The spaceborne LiDAR system GLAS on board of ICESat satellite platform was launched in January, 2003. GLAS sensor emits pulses of 1064nm monochromatic light operating at $40 \mathrm{~Hz}$ onto the surface recording the returning energy as a function of time, which is full-waveform LiDAR [1]. The footprint of GLAS is approximately $65 \mathrm{~m}$ in diameter, and pulses were spaced at a 170m interval [1]. The canopy height can be estimated by analysing 
vertical components of GLAS, providing that topographic variation of the study area is low. However, the vertical components of GLAS in steeply sloping regions are complex to interpret in terms of canopy height [121] and an additional correction factor is required. Lefsky et al. [175] used topography information obtained from shuttle radar topography mission (STRM) to offset slope effects. Their correction model could explain up to $68 \%$ of variance in field-measured canopy height. The slope corrected GLAS with STRM [175] is a standard method to derive canopy height information [37, 173, 28, 238, 26]. GLAS dataset has also been used to estimate biomass in a peat swamp forest that was remotely located and difficult to access [28]. In this study, GLAS was normalised by STRM, then the calibration was applied using airborne LiDAR survey. The calibrated GLAS could explain $61 \%$ variances of canopy height and above ground biomass. GLAS has also been used to estimate forest biomass on a regional scale [37, 26]. For example, Boudreau et al. [37] used both airborne LiDAR and GLAS to estimate biomass over 1.27 million $\mathrm{km}^{2}$ temperate and boreal forest in Quebec. Ultimately, GLAS can be used to estimate biomass on a global scale [25, 24, 173, 238]. It has been used to estimate biomass of tropical Africa [25], carbon emissions from pan-tropics (including tropical Africa, America and Asia), global-scale biomass map [173, 238]. GLAS was used to map biomass of tropical forests along with MODIS and field inventories[25, 173, 24, 272]. With calibration between satellite sensors (GLAS and MODIS) and field inventories, global-scale biomass can be derived and crossvalidated $[25,173,24]$. However, it is still difficult to estimate biomass on a global scale using GLAS due to its sparse spatial coverage [173, 238]. Simard et al. [238] resolved this problem using random forest regression between GLAS derived canopy height and other variables, such as annual precipitation. In this case, the random forest regression model filled the gap which the GLAS sampler had not covered, so forest biomass was mapped in a global scale [238]. 
Airborne LiDAR has become a practical tool for forest mensuration which is increasingly utilised in forest inventory programmes at national and regional scales [191]. Airborne LiDAR emits pulses of monochromatic light onto the surface, and measures the returning signals in full-waveform. Older airborne LiDAR systems automatically converts backscattered energy in the returning full-waveform into the discrete point clouds due to its heavy memory costs. As technology developed, airborne LiDAR systems have started to record returning energy continuously, so full-waveform profiles are now available, but rarely used operationally. With the recent development of global positioning system and inertial navigation system, which measure the position (roll, pitch and yaw) and altitude of aircraft with high precision. For example, NASA's experimental LiDAR system, called scanning Laser imager of canopies echo return, was successfully integrated with global positioning system, thus demonstrating that airborne LiDAR can locate objects at $\sim 1 \mathrm{~m}$ scale resolution in both vertical and horizontal directions $[34,172,174,189]$. The figure 1.1 describe a pictorial illustration of the reconstruction of forest structures using LiDAR.

Airborne LiDAR analysis approaches can be classified into two groups: area-based and ITC-based.

The area-based approach compares plot-level LiDAR attributes with ground measurements [189]. The important concept of area-based approach is that summary statistics derived from the discrete LiDAR returns represent the structure components of vegetation. Specifically, area-based methods are based on statistical summaries of the vertical height components of LiDAR point cloud in a selected area $[185,187,189]$. There has been much discussion about the minimum density of points needed to derive reliable statistics. , LiDAR Consensus is that point densities need to be greater than 0.1 point $/ \mathrm{m}^{2}$, and the size of the ground plot for calibration should not be less than $200 \mathrm{~m}^{2}$ [189]. In reality, these are low point densities for modern ALS to produce; it is not uncommon to obtain imagery with 10 point $/ \mathrm{m}^{2}[153,128,86,89]$. Previous studies have demonstrated that forest parameters, such 
as basal area, mean canopy height and mean canopy volume, can be predicted using the area-based approach. Magnussen and Boudewyn [185] showed that the distribution of canopy height is linked with the vertical profiles of foliage area. In this study, the fraction of leaf area and LiDAR derived canopy height distribution had very good correlation. Means et al. [192] estimated canopy height, basal area and volume using linear regression between LiDAR derived metrics and field measurements. Lefsky et al. [174] demonstrated that LiDAR can be used to reconstruct forest height, cover and structures. They predicted above-ground biomass, leaf area index (LAI) and DBH in 22 plots. The results showed that area based approach could predict these parameters very accurately. More recently, non-parametric approaches have been investigated to characterise relationships between forest biophysical parameters and LiDAR derived metrics [188, 206, 136, 161]. For example, nearest neighbour (NN) imputation framework is the popular non-parametric regression to estimate forest biophysical parameters using LiDAR [206, 136]. In the NN imputation framework, an estimate for an observation is imputed from a set of nearest neighbours. The set of nearest neighbour is defined from independent predictors where the relationship between the observations and predictors are known. Hudak et al. [136] used nearest neighbour imputation to estimate forest attributes (basal area and tree density) from 12 selected LiDAR metrics. Moreover, NN imputation using LiDAR and field plots has been implemented to estimate species-specific stand level attributes, such as volume, stem number, basal area, basal area median diameter and tree height, in National forest inventories of Finland [206, 189].

ITC based methods, on the other hand, detect individual tree tops or crowns, then use allometric equations to predict forest properties [189]. ITC delineation is the key step in individual tree based methods. ITC delineation methods can be classified as two different groups: canopy height model (CHM) based methods and LiDAR point cloud based methods. The details of the ITC delineation strategy will be explained in Section 1.4. In this section, we will keep our focus on predicting forest attributes. ITCs typically provide tree height, 
crown diameter and vertical height profiles. These LiDAR derived variables are used to estimate DBH, biomass and stem wood by regression analysis[221, 144, 220, 87]. For example, Popescu et al. [221] extracted ITCs from LiDAR and derived tree height and crown diameters. They modelled the linkage between tree height, and crown diameter with biomass through linear regression with field measured biomass. ITC-level LiDAR variables can also be used to classify species $[38,205]$. Species recognition using LiDAR could lead to more accurate forest attributes because these attributes are usually species-specific [144]. For example, Ørka et al. [205] extracted structural and radiometric features by analysing ITC vertical profiles. They achieved $88 \%$ of accuracy for large trees using these features.

This section has reviewed different LiDAR platforms and their applications on ecological research. GLAS can be used to estimate regional- and global scale biomass and carbon storage, while airborne LiDAR data can be used to provide more precise maps of forest structural attributes, either by area-based or ITC-based approaches. These two platforms are complementary, with airborne LiDAR being used to calibrate GLAS data, thus providing large-scale biomass estimation. This thesis will focus on improving ITC delineation and exploring its applications for species mapping at the ITC level.

\subsection{Hyperspectral imagery and spectranomics}

Hyperspectral imaging spectrometers measure energy in the electromagnetic spectrum from visible to shortwave infrared. Transitions between the sets of energy levels in molecules give rise to the absorption and emission of electromagnetic radiation [129]. The transition between molecular energy levels can be modelled and distinguished by modern spectroscopic techniques (Rotational, Vibrational, Electronic, Raman spectroscopy, etc.) [129]. Molecules absorb visible and near-infrared parts of the spectrum when vibrational or electronic energy changes. The radiation from the sun is incident on a surface, chemical constituents of the surface absorb, transmitted or backscattered radiation in some wavebands. Figure 1.2 


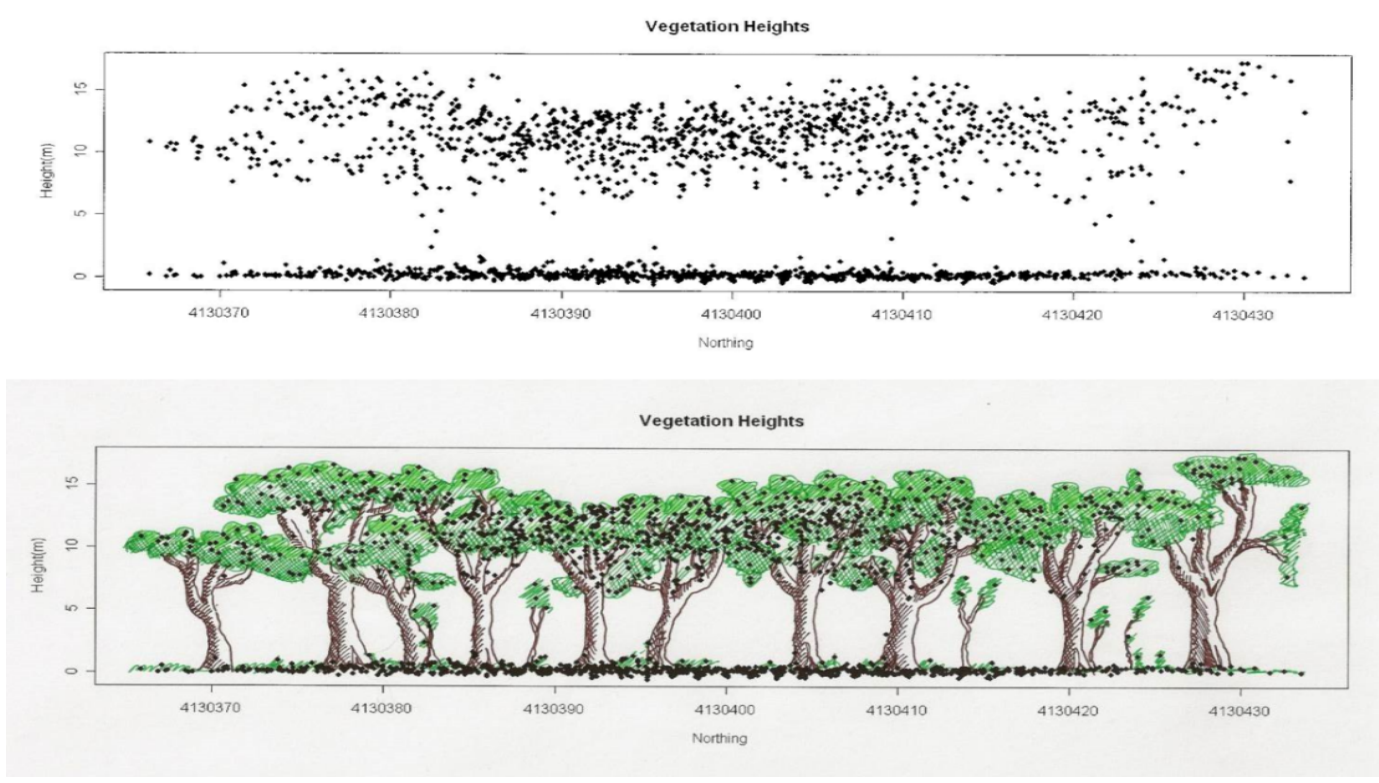

Fig. 1.1 An example of airborne LiDAR point cloud (a) and a pictorial illustration of the reconstruction of forest structures (b) (provided by Dr. Simonson).

describes the structure of hyperspectral data and its collection process. As shown in Figure 1.2, imaging spectrometer measures the amount of the surface radiance such that one can calculate the surface reflectance if one knows the radiance of incoming light [2]. Therefore, we can identify the chemical properties of a surface as the molecular constituents dictate the spectral properties of the surface [2].

As hyperspectral sensors measure the reflectance of the ground objects, hyperspectral imaging is an excellent tool for identifying physical and chemical properties of canopy structures and this is key for understanding physiological processes of vegetation. Foliage cover in the canopy is an important ecological indicator for species competition and net primary productivity [94] and spectral indices or statistical calibration reveal biophysical and chemical properties of canopy leaves. Spectral indices of physiological function are usually based on a ratio between two or more spectral bands. For example, Gamon et al. [103] derived the photochemical reflectance index (PRI) to monitor photosynthesis efficiency of vegetation, while Gitelson et al. $[112,110]$ developed Carotenoids reflectance 
and Anthocyanins reflectance indices. These spectral indices provide a practical means of monitoring temporal variation of photosynthetic light use efficiency (LUE), which is the ratio between carbon uptake and light absorbed by vegetation, thus providing actual carbon flux changes [105]. Specifically, PRI can explain $42 \%, 59 \%$ and $62 \%$ of the variations of LUE at the leaf, canopy and ecosystem levels respectively making it an efficient tool for monitoring and modelling ecosystem energy circulation in different scales [105]. However, there is still uncertainty about LUE estimations because of species diversity, which makes precise mapping of forest attributes challenging [17].

As hyperspectral imaging techniques develop, spatial resolution has become fine enough to estimate leaf traits from statistical analysis linking hyperspectral imagery with fieldmeasured canopy leaf chemistry. Ustin et al. [253] demonstrated the concept of statistical calibration of leaf physical and chemical traits using hyperspectral imagery. Asner and Martin [17] introduced spectranomics to monitor the canopy chemical and taxonomic diversity of tropical forests. This spectranomics approach links field measurements of foliar chemistry with airborne hyperspectral signals, thus establishing a statistical relationship between chemical traits and spectral signals using parametric or non-parametric regressions. For example, Feilhauer et al. [90] suggested brightness normalised partial least square to predict chemical contents from hyperspectral imagery. This method used the euclidean distance of spectral angle to normalise brightness, and showed better performance by $3.6 \%$ compared to conventional partial least square regression. Spectranomics also showed that the environmental effects on chemical variations were relatively small compared to integrated chemical signatures of each species. This allows for canopy species to be identified by hyperspectral imagery [17]. Spectranomics has a potential to revolutionise ecological research, as it can provide canopy level leaf chemistry and species information. Moreover, with the combination of LiDAR and hyperspectral imagery in a multi-sensor approach, spectranomics can map 
ITCs, foliar chemical diversities, and species distribution [17]. The following section will investigate the usage of this multi-sensor spectranomics.

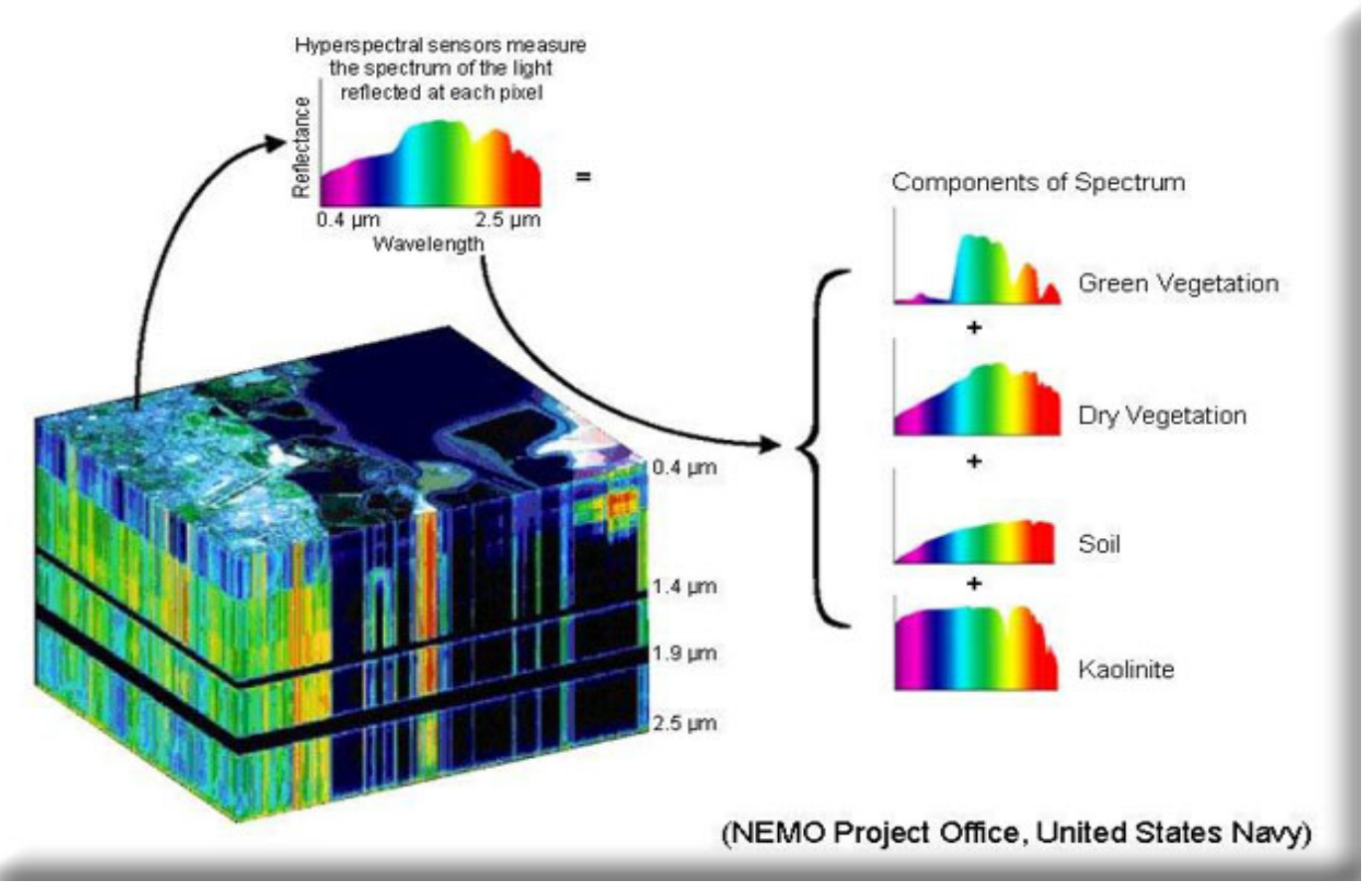

Fig. 1.2 The basic concept of hyperspectral data.

\subsection{Ecological applications of LiDAR and hyperspectral imagery fusion}

Combining hyperspectral images and LiDAR information is a powerful technique for forest ecology. LiDAR imagery can be used to create a Digital Surface Model (DSM) - a 3D surface shape of the research area. As the DSM represents the actual geometrical shape of the forest, it can be used for removing geometrical errors of hyperspectral images. Since hyperspectral data consist of $2 \mathrm{D}$ focal images of the area such that the images do not contain any surface geometrical information, hyperspectral data can only provide distorted information about the surface. This can be corrected using the DSM obtained from LiDAR. In addition, LiDAR 
point clouds can be used to delineate tree crowns such that hyperspectral images can be analysed at an individual crown scale $[17,71]$. Hyperspectral imagery depends upon physical and chemical properties of each crown. Asner and Martin [17, 18] have found that some species in tropical forests have unique spectral and chemical signatures such that individual species can be identified using hyperspectral and LiDAR information.

Remote sensing provides a new approach for environmental monitoring over large spatial scales. Since chemical and spectral signatures of individual species are unique, the distribution of invasive species and native species of the upper story canopy trees in a forest can be monitored $[16,13]$. For instance, the ecosystem of Hawaiian forests has experienced rapid changes following the introduction and expansion of alien species. Non-native species compose almost half of all species in Hawai'i and 120 species are regarded as highly invasive [16]. One of the invaders is Myrica faya, which was firstly observed in 1961 and expanded to cover over 34,000ha by 1985 [255]. M. faya is an actinorhizal nitrogen fixer, which has facilitated its rapid spread in mountains in which soils are rich in phosphorus but contain little nitrogen [255]. Monitoring aspects of ecosystem changes, including the spread of invasive species, is important to protect native biodiversity. Asner et al. [16] showed that invasive species and native species distributions in Hawaiian forests could be monitored using hyperspectral data. Furthermore, Asner et al. [18] proved that both invasive and native species can be identified at an individual tree scale by combining LiDAR and hyperspectral data. Based on these technological breakthroughs, Asner et al. [8] observed the changes in 3D structures of native forests caused by invasive species in Hawai'i. Further research has been conducted to determine the robustness of the results considering inter-seasonal spectral and chemical variation, since forest seasonality and phenology may influence spectral and chemical properties of species [124, 241].

Airborne remote sensing may help resolve an important question in climate change: how will drought affect tropical rain forests? The role of tropical ecosystems in the global 
carbon cycle is important in the context of climate change and its consequences [72]. One climate/carbon-cycle coupled model controversially predicted that carbon-cycle feedbacks would accelerate global warming with the loss of extensive areas of Amazonian forests by 2100 [72]. The loss of Amazonian forests predicted in the model was related with predicted increases in the frequency of El-Niño Southern Oscillation events, which caused drought over Amazonian forests [113]. The model also assumed that all species in the rain forest had the same physiological response to water shortage, whereas in reality a range of responses is likely. Although a number of studies of the impact of drought on Amazonian forests have been conducted, consensus has not been reached [137, 234, 113, 186, 218, 10, 235]. Observational research based on analyses of satellite imagery showed that Amazonian forests were greening-up in years of severe drought events [234]. This implies that Amazonian forests may be resilient to seasonal drought [137, 234, 218]. However, satellite-based spectral information may not provide enough information to measure the drought impacts on Amazonian forests because spatial resolution is too low [10,235] and variation in individual species responses are not quantified. Furthermore, a recent study conducted by Morton et al. [198], who used ICESat/GLAS and a sophisticated radiative transfer modelling, showed that effect on vegetation indices could be an artefact of sun-sensor geometry. Studies based on permanent ground plots over Amazonian forests indicate that large trees and species with low wood density are particularly vulnerable to drought, such that functional composition of Amazonian forests could be changed toward denser-wooded trees [218], perhaps without a large-scale loss of rain forest.

Hyperspectral and LiDAR information can help researchers understand the variety of species responses to climate change, since spectral and chemical signatures of undamaged and damaged trees are distinguishable. Gregory Asner of the Carnegie Institution ${ }^{1}$ released initial results of the impact of 2010 drought on Amazonian forests, which were obtained from hyperspectral images and LiDAR information over 500,000 hectares of the forests [6]

\footnotetext{
${ }^{1}$ http://dge.stanford.edu/labs/asnerlab/
} 
(Figure 1.3). Combining hyperspectral and LiDAR data, they detected leafless branches and stressed trees at an ITC level. However, at the time of submitting this thesis, a full paper explaining these preliminary results has not been published.

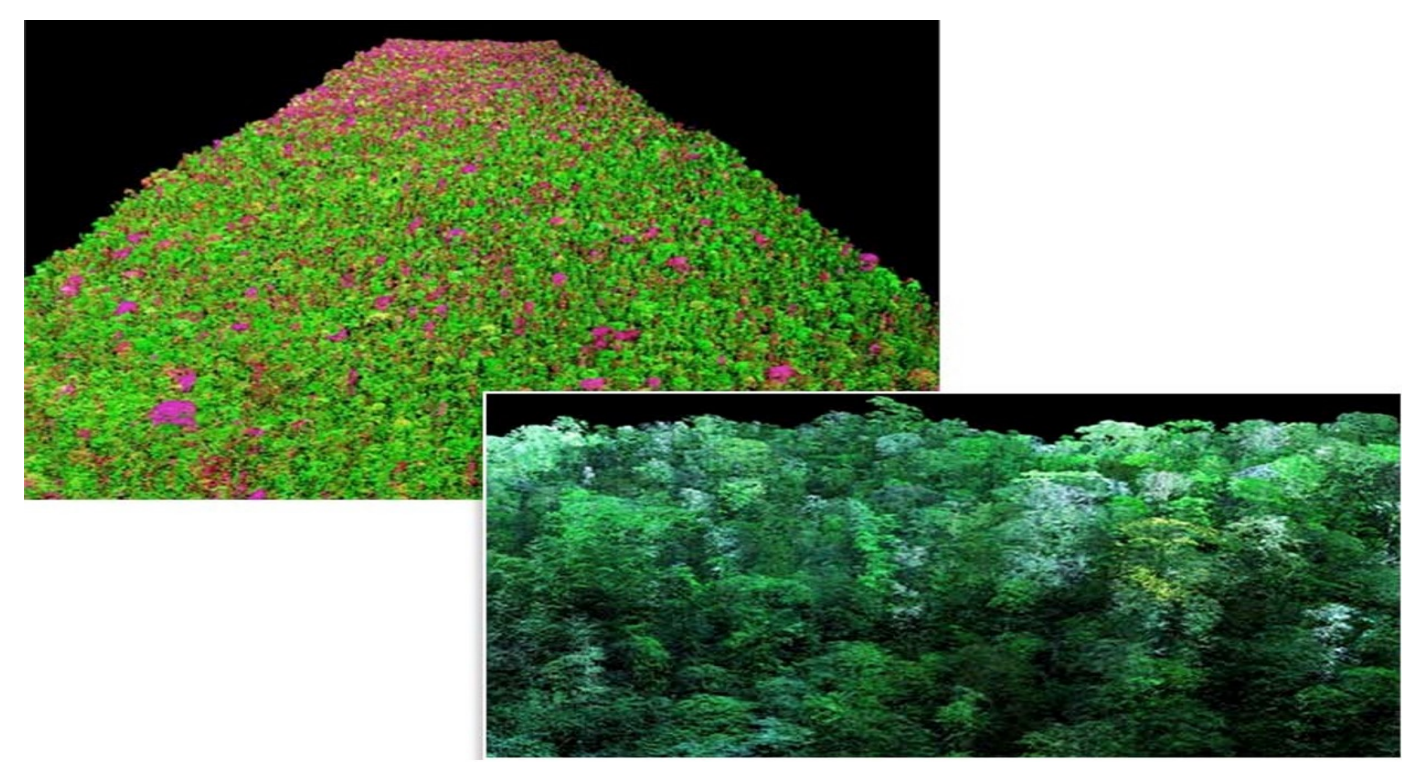

(Obtained from AAAS website: http://news.sciencemag.org/sciencenow/2012/12/widespread-devastation-found-in-.html)

Fig. 1.3 The impacts of 2010 drought on Amazonian forests. The red colour indicates stressed trees (top image), whereas white indicates leafless branches (bottom image).

\subsection{Technical difficulties of hyperspectral and LiDAR fu- sion}

Fully integrated hyperspectral and LiDAR imagery has enormous potential in ecological research. However, technical difficulties in LiDAR and hyperspectral imagery fusion make its application challenging. Co-alignment of LiDAR and hyperspectral imagery is a key process to achieve high-resolution species mapping and spectranomics. However, the georeferencing system of LiDAR and hyperspectral imagery is often inaccurate, causing mis-alignments that need to be addressed. Even if LiDAR and hyperspectral imagery were collected simultaneously, inaccurate flight positioning and boresight calibration systems cause inaccurate 
georeferencing. In addition, hyperspectral imagery needs orthorectification to offset topographic distortion. Topographic distortion is usually corrected by a digital elevation model. However, it may still remain because of the coarse resolution of the digital elevation model.

\section{Image registration}

Table 1.1 The list of algorithms that co-align multi-sensor imagery

\begin{tabular}{c|l|c}
\hline Category & \multicolumn{1}{|c|}{ Method } & Reference \\
\hline \hline \multirow{4}{*}{ Feature-based } & Manual Selection & {$[101,164]$} \\
\cline { 2 - 3 } & Scale Invariant Feature Transform & {$[182,178]$} \\
\cline { 2 - 3 } & Speed-Up Robust Feature & {$[30,242]$} \\
\cline { 2 - 3 } & Feature Segmentation & {$[115,114]$} \\
\hline \multirow{2}{*}{ Area-based } & Normalised Cross Correlation & {$[194,164]$} \\
\cline { 2 - 3 } & Mutual Information & {$[59,194,180]$} \\
\hline
\end{tabular}

Image registration is the technique used to co-align images taken from the same target. Image registration is used to find a transformation function $\phi$, which is defined by a set of parameters, such that a template image $T$ is aligned with a reference image $R$. Table 1.1 shows the lists of image registration algorithms. Image registration techniques can be categorised into feature-based and intensity-based methods.

Feature based methods rely on common features extracted from reference and template images. These common features can be defined using manual feature selection [101], SIFT [182], SURF [30, 242] and feature segmentation[115, 114]. Manual selection works by finding Ground control points (GCPs) manually by visual inspection [101, 164]. However, the manual selection of GCPs is time-consuming and labour intensive. Feature descriptor is an alternative way to find common features automatically. Scale-invariant feature transform (SIFT) is a popular feature descriptor to exploit key features [182] using gradient information to find common features of reference and template images to perform optimal transformation. The speeded-up robust feature transform finds common features using an approximation of hessian matrix, then computes an optimal transformation function between template and 
reference images [30, 242]. Goncalves et al. $[115,114]$ suggested a feature segmentation method that segments both reference and templates images into several sub-images, then finds possible matches between these sub-images.

Intensity based methods involve maximising similarity in intensity values between reference and template images. There are two popular intensity based registration methods: normalised cross correlation [194, 164] and mutual information [59, 194, 180]. The normalised cross correlation method computes the cross correlation between reference and template images, then finds optimal parameters of transformation [164]. However, remote sensing images taken from different sensors have systematically different intensity values. Under this condition, normalised cross correlation fails to find a meaningful transformation. Mutual information $[59,194,180]$ is designed to register images taken from different sensors, such that intensities of two images are systematically different. Mutual information computes a joint histogram of template and reference images. The joint histogram changes as the alignment between template and reference images change. Mutual information finds an optimal transformation by measuring the sharpness of this joint histogram. Intensity methods are intrinsically fully automatic, as these methods need not extract common features. However they are often mathematically ill-posed, in the sense that registration transformation might not be unique and registration results could be significantly influenced by small changes in the images [194].

\section{Individual tree crown delineation}

Accurate tree delineation from multi-sensor imagery is another important technical issue. Recognising ITCs plays a important role in tracking invasive species and understanding species distribution [16]. Forest biomass can be more accurately measured if species are known for each tree, using ITC delineation [189]. Airborne LiDAR is a popular tool to delineate ITCs as it provides 3D structure of the forest in question. Table 1.2 shows the lists of 
Table 1.2 The list of methods that delineate individual trees from LiDAR.

\begin{tabular}{c|l|c|c|c}
\hline Category & \multicolumn{1}{|c|}{ Method } & Forest type & Accuracy & Reference \\
\hline \hline \multirow{4}{*}{ CHM-based } & Moving window filtering & coniferous & n/a & {$[139]$} \\
\cline { 2 - 5 } & Watershed segmentation & Savanna & $64 \%$ & {$[60]$} \\
\cline { 2 - 5 } & Multiscale edge segmentation & temperate & $23-35 \%$ & {$[39]$} \\
\cline { 2 - 5 } & Attentive vision method & coniferous & $83 \%$ & {$[207]$} \\
\hline \multirow{3}{*}{ Point cloud - based } & $k$-means clustering & coniferous & $60 \%$ & {$[197]$} \\
\cline { 2 - 5 } & Relative spacing & coniferous & $94 \%$ & {$[179]$} \\
\cline { 2 - 5 } & Recursive normalised cut & coniferous & $60 \%$ & {$[224]$} \\
\hline
\end{tabular}

algorithms, and forest types, where these algorithms are tested as well as their accuracy. Note that accuracy in Table 1.2 is not suitable for comparison because their evaluation methods were different and conducted in different study sites; we address this issue in Chapter 3 by using benchmark datasets and algorithms.

Conventional LiDAR-based ITC delineation algorithms convert LiDAR point clouds into digital surface models (DSM), then normalise the DSM with terrain elevation in a CHM. CHM is used to exploit local maxima, which are assumed to be tree tops. As shown in Table 1.2, these methods include moving window filtering [139], watershed algorithm [60], multi-scale edge segmentation [39] and attentive vision method [207]. Moving window filtering method [139] applies $n \times n$ size local maxima search filter to each pixel, then use region growth algorithm to delineate ITCs. Hyyppa et al.[139] used a moving window filtering algorithm to estimate individual tree parameters in a coniferous forest in Finland. Although this study successfully estimated stem volume, mean height and basal area, it did not show how many ITCs were correctly segmented. Watershed algorithm is another way to delineate ITCs. It uses an inverted CHM, which is analogous to a drainage basin, so local minima can be found following gradient flows of the inverted CHM [60]. Chen et al. [60] suggested a marker-controlled watershed algorithm. In this case, the watershed algorithm is initiated from markers, which are pre-defined so that markers growing towards their neighbours are analogous to pouring water inside. This method achieved $64 \%$ accuracy 
in a Savanna woodland. Another approach is multi-scale edge segmentation [39]. This method filters the CHM by examining textures at different scales, thus delineating ITCs. This algorithm was tested in a temperate forest and achieved 23-35\% accuracy. Attentive vision method [207] extracts seeding points by a feature descriptor, then applies region growth towards neighbouring points, thus finding boundaries of each ITC. The method was evaluated in a coniferous forest and showed $83 \%$ accuracy.

CHM based methods are simple and computationally fast. However, these algorithms have intrinsic problems. Since CHM represents the canopy surface, it is impossible to detect sub-canopy trees, unless additional steps are included to analyse LiDAR point cloud statistics [86]. The performance of tree delineation depends strongly on the choice of smoothing parameter. Finding an optimal value for the smoothing parameter reduces the benefits of automation. As the CHM is derived by interpolation of LiDAR point clouds, the actual geometry of forest is distorted by interpolation induced artefacts. For instance, the CHM tends to underestimate tree heights due to the interpolation process.

3D point clouds based tree segmentation methods could alleviate CHM related problems. These methods include $k$-mean clustering [197], relative spacing [179] and recursive normalised cut [224]. Morsdorf et al. [197] used $k$-mean clustering to delineate ITCs. $k$-mean clustering is looking for $\mathrm{k}$ clusters where each point belongs to the cluster with the nearest mean. This approach was validated in a coniferous forest and detected $60 \%$ of ITCs. Method relative spacing is to find ITC starting from the tree top [179]. From a seeding point at the tree top, LiDAR points are clustered sequentially, from the highest to the lowest. Each seed point merges nearby points, and exclude points from different trees based on their horizontal spacing at different height interval. This method was evaluated in a coniferous forest and achieved 94\% accuracy. Another method known as recursive normalised cut was suggested by Reitberger et al. [224]. Normalised cut finds an optimal way of separating points into two disjoint clusters based on a similarity between the points. To find several 
trees, this algorithm applies the normalised cut to the clusters repeatedly until certain criteria are reached. The normalised cut algorithm was validated in a coniferous forest, and detected $60 \%$ ITCs accurately. However, 3D point cloud based algorithms are still less popular than CHM based methods, as these algorithms are computationally heavy.

\section{Species classification using machine learning techniques}

Table 1.3 The list of methods that classify and map tree species from either hyperspectral imagery or hyperspectral imagery alongside LiDAR.

\begin{tabular}{c|l|c}
\hline Category & \multicolumn{1}{|c|}{ Method } & Reference \\
\hline \hline \multirow{2}{*}{ Parametric } & Linear discriminant analysis & {$[67,77,93]$} \\
\cline { 2 - 3 } & Maximum likelihood & {$[67,74,77]$} \\
\hline \multirow{3}{*}{ Non-parametric } & Spectral angle mapper & {$[67,63]$} \\
\cline { 2 - 3 } & Random forest & {$[163,76,200]$} \\
\cline { 2 - 3 } & Support vector machine & {$[74,77,146,76,79,78,71]$} \\
\hline
\end{tabular}

Species classification using multi-sensor imagery is an important technical issue, which can provide a practical tool for monitoring species diversity and calibrating heterogeneity of ecosystems at large scales [93]. In combination with ITC delineation described in the previous section, this technique could inform species-specific biophysical parameters at ITC level [78]. Hyperspectral imagery measures the radiance of a target in narrow spectral bands ranging from visible to shortwave infrared, so it can be used to discriminate species by linking a particular spectral region to a specific characteristic of the species $[17,76]$. Supervised machine learning techniques have been successfully used to map species at pixel level in different types of forests $[67,17,77,74,146,76,71,93,78]$. Table 1.3 shows the list of methods that have been used for species classification.

Supervised machine learning techniques can be categorised into parametric and nonparametric methods. Parametric methods assume that the probabilistic distribution of each class is known, so it estimates the probabilistic density function of each class using parameters computed by training samples of the class. Parametric methods commonly used in remote 
sensing include maximum likelihood $[67,74,77]$ and linear discriminant analysis $[67,77,93]$. The maximum likelihood method $[67,74,77]$ assumes that each class is normally distributed. Mean and covariance are computed using training samples of each class, such that the posteriori probabilistic density is estimated. The linear discriminant analysis $[67,77,93]$ is another parametric method commonly used in remote sensing. In this method, a classification function is built using the covariance matrix and predictor variables pooled within each class. Although these parametric methods have been used to classify tree species from hyperspectral imagery, they are influenced by the Hughes phenomenon [138], i.e. the number of samples required for each class must be equal or larger than the number of feature dimensions. Therefore, these methods may not be suitable for species classification if the number of samples per each species is limited.

Non-parametric methods do not assume probabilistic distribution of each class. Nonparametric methods commonly used in remote sensing include spectral angle mapper [67, 63], random forest $[163,76,200]$ and support vector machine $[74,77,146,76,79,78,71]$. The spectral angle mapper method computes the spectral angle between reference and target spectra. Since it only compares the direction of spectrum, it is not influenced by the magnitude, thus providing robust classification results. Another method known as random forest $[163,76,200]$ is a popular tool used for species classification. Random forest constructs hundreds of decision tree models using randomly selected inputs of features, then combines the outputs via voting. The support vector machine $[74,77,146,76,79,78,71]$ is a well established method for species classification. This method searches an optimal hyperplane separating data into different classes. Support vector machine showed a superior or at least comparable performance as other methods for species classification $[74,77,146,76,79,78$, 71]. Throughout this thesis, we used support vector machines to classify tree species because it outperformed other methods in many studies. 


\subsection{Contribution}

The main body of this thesis consists of four chapters investigating technical solutions of multi-sensor remote sensing for ecological research. At the time of Ph.D. thesis submission, each chapter has either been published or is currently under review. The second chapter was published in IEEE transactions on Geoscience and Remote Sensing [171]. The third and fourth chapters are currently under review in Remote Sensing [168] and IEEE Journal of Selected Topics in Applied Earth Observations and Remote Sensing [169], respectively. A summary of the fifth chapter was published in Proceeding IGARSS 2015 [170]. A short summary of each chapter is provided below.

\subsubsection{Non-parametric image registration of airborne LiDAR, hyper- spectral and photographic imagery of wooded landscapes}

In Chapter 2, we focus on the image registration problem to co-align remote sensing datasets taken from different sensors. This work is the result of a collaboration with my supervisors Carola-Bibiane Schönlieb and David Coomes - and a postdoc in Cambridge - Xiaohao Cai [171].

Multi-sensor imaging provides different features of vegetation, such that it collectively provides detailed information of the ecological process [17]. Images taken from different sensors need to be co-aligned, but suffer from translation, rotation, scale mismatches, and lens distortion and topography that cause complex mismatches between the images. Correcting misalignment between the images is needed in image registration pipelines. Registration algorithms typically used in remote sensing find a set of registration parameters, which are linked to scale, translation and rotation transformations [164]. However it is hard to correct complex distortion using a fixed set of transformation parameters [194]. In this chapter, non-parametric registration based on a variational formulation is introduced. Using 
this approach, the registration transform is no longer parameterised, but instead a cost function designed for image registration is minimised to find an optimal transformation. Nonparametric registration measures not only similarity between images but also regularity of the transformation in the calculated cost function, thus alleviating the ill-posedness of registration problem. This chapter develops a cost function with normalised gradient field similarity measure and curvature regularisation, called NGF-Curv [98, 118]. The normalised gradient field similarity measure works by maximising the linear dependency between the gradients of images [118]. Curvature regularisation ensures the smoothness of the transformation [98]. This chapter demonstrates the efficiency of NGF-Curv regularisation using LiDAR and hyperspectral imagery and aerial photographs. This approach outperforms state-of-the-art approaches [30, 242, 59, 194, 180], assessed by calculating the absolute difference between registered hyperspectral imagery $(T)$ and LiDAR intensity image $(R)$, as shown in Figure 1.4.

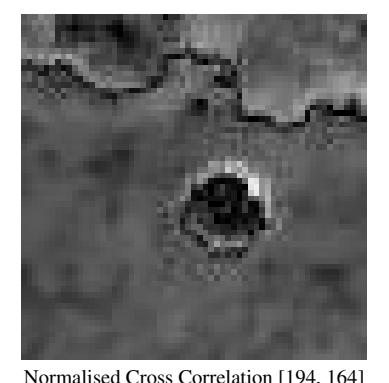

Normalised Cross Correlation [194, 164]

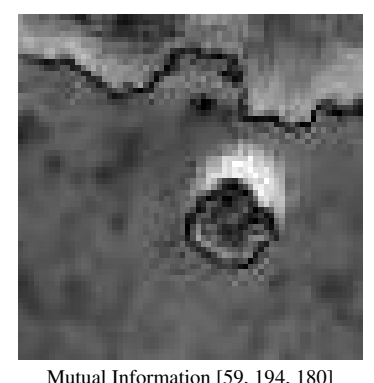

Mutual Information [59, 194, 180]

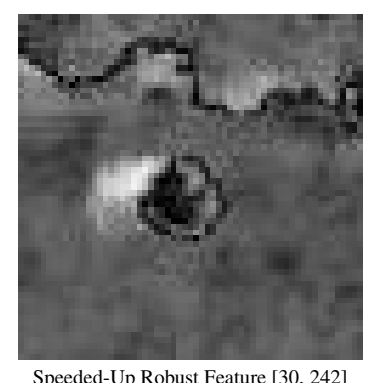

Speeded-Up Robust Feature [30, 242]

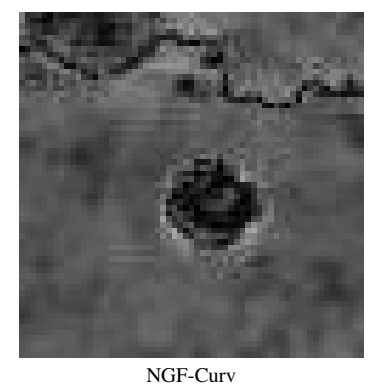

NGF-Curv

Fig. 1.4 The absolute difference between a registered hyperspectral image $(T)$ and a LiDAR intensity image $(R)$. (i.e. $|T-R|$ )

\subsubsection{D individual tree Segmentation of integrated airborne LiDAR and optical imagery using normalised cut with priors}

In Chapter 3, a 3D ITC delineation method using hyperspectral and LIDAR fusion will be investigated. A normalised cut based ITC delineation, called MCNCP-RNC is designed to reconstruct ITCs from LiDAR along with hyperspectral imagery. The work was developed in 
collaboration with my supervisors Carola-Bibiane Schönlieb and David Coomes and three postdocs in Cambridge - Xiaohao Cai, Jan Lellmann, Michele Dalponte [168].

Airborne multi-sensor imaging can be used to delineate ITCs. The LiDAR component provides a point cloud reflecting the 3D structures of the vegetation, while the hyperspectral imagery provides physical and chemical traits of vegetation canopy. The combination of LiDAR and hyperspectral imagery could delineate ITC effectively in a superior manner in comparison to traditional methods [78]. Conventional ITC delineation methods work with interpolation of the LiDAR point cloud [139, 60, 39, 207]. These methods suffer interpolation related artefacts, thus providing inaccurate tree height and volumes. In addition, sub canopy trees are impossible to detect because the methods are based solely on canopy surface geometry.

This chapter develops a new 3D ITC delineation method based on normalised cut framework [237], called MCNCP-RNC. Normalised cut finds an optimal way to separate points into different clusters based on similarity weights between the points. Since the weights can be defined using hyperspectral imagery alongside LiDAR, normalised cut framework is ideal for fusing different types of remote sensing dataset. In addition, correlation constraint between priors and optimal normalised cut partitioning is introduced to ensure more realistic ITC delineation [133].

The performance of MCNCP-RNC was validated using data from three different types of forests. The validation was conducted by software provided by NewFor project [89]. The performance of MCNCP-RNC outperformed an interpolation-based method called TIFFS [60], which was the most accurate of the canopy-height-model-based approaches we compared. It proved to be a good algorithm for distinguishing upper canopy trees, even in complex deciduous forests. Although the MCNCP-RNC approach found a few sub-canopy trees, the detection rate was still low. The hyperspectral imagery improved ITC delineation at the expense of introducing more false positives. 
Figure 1.5 gives an example of MCNCP-RNC segmentation based on the NewFor benchmark data. An advantage of the proposed delineation approach is that it provides 3D information of individual trees. This information can be used to estimate various ITC parameters, including crown area, crown shape and tree height, which may help to identify species and estimate forest biomass more accurately.

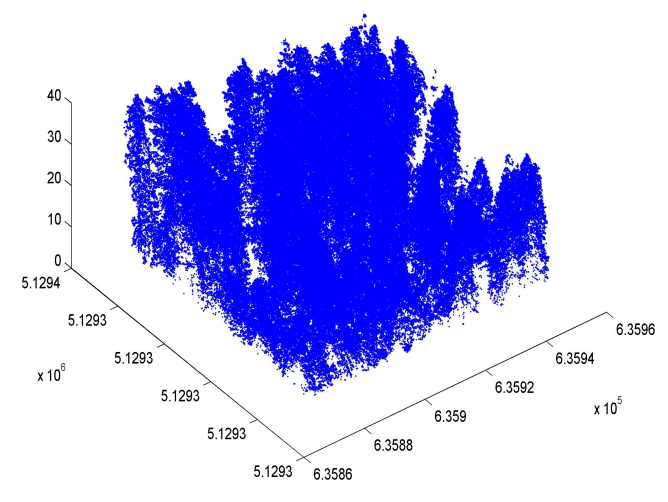

(a)

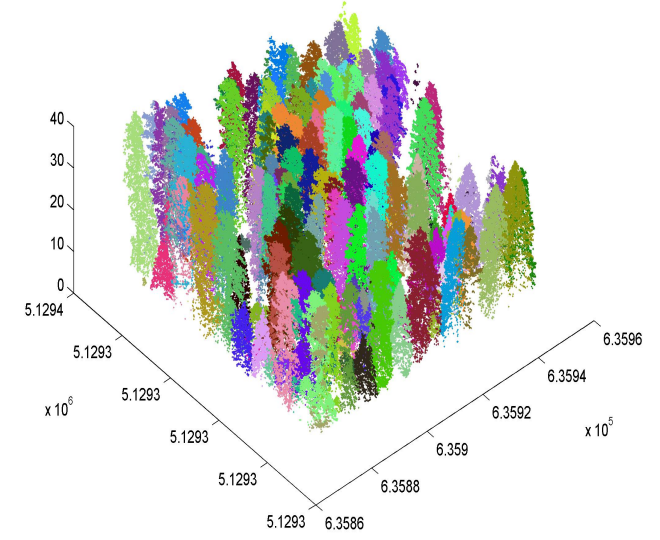

(b)

Fig. 1.5 An example of MCNCP-RNC segmentation. (a) indicates a LiDAR point cloud and (b) shows ITCs in different colours.

\subsubsection{Individual tree species classification from airborne multi-sensor imagery}

Connecting the methods developed in the previous two sections, Chapter 4 develops a workflow for species classification using LiDAR and hyperspectral imagery. This work is the result of a collaboration with my supervisors - Carola-Bibiane Schönlieb and David Coomes - and their postdocs Xiaohao Cai, Jan Lellmann and Michele Dalponte [169].

Mapping species at ITC level provides fundamental understanding of forest responses to global climate change and a practical mean to monitor species distribution patterns [14]. 
Mapping species at ITC level requires a large amount of effort in conventional ecological survey methods, which generally rely on information collected from small inventory plots [132]. LiDAR and hyperspectral imagery provide detailed geometrical and optical structures of forest needed for precise mapping of individual tree species at large scales. In this chapter, we introduce a systematic workflow to detect tree species at ITC level using hyperspectral imagery and LiDAR. This workflow incorporates the novel algorithms developed in Chapters 2 and 3. The first step is the registration of LiDAR and hyperspectral imagery. The second step is reducing the dimensions of hyperspectral imagery and extracting meaningful features using robust PCA [53]. The third step includes ITC delineation using LiDAR and hyperspectral imagery. The fourth step contains pixel-level species classification using hyperspectral imagery. Finally, ITC-level species map is constructed using the majority voting method $[93,78]$.

The developed workflow for mapping species at ITC level was tested in a temperate deciduous woodland near Oxford, UK. The validation was conducted by validation software for ITC delineation and confusion matrix analysis $[89,74]$. The accuracy of species classification at pixel and ITC levels was examined with ground truth. Our method achieved $89 \%$ overall accuracy for pixel-level species classification, while conventional PCA based classification [145] showed $84 \%$ overall accuracy.MCNCP-RNC correctly segmented only $40 \%$ of canopy trees. ITC-level species classification accuracy was $66 \%$ for correctly delineated trees. Figure 1.6 shows a map of species as classified at ITC level in comparison to ground data: visual comparison suggests that the species distribution patterns of segmented trees and ground truth were well-matched. 


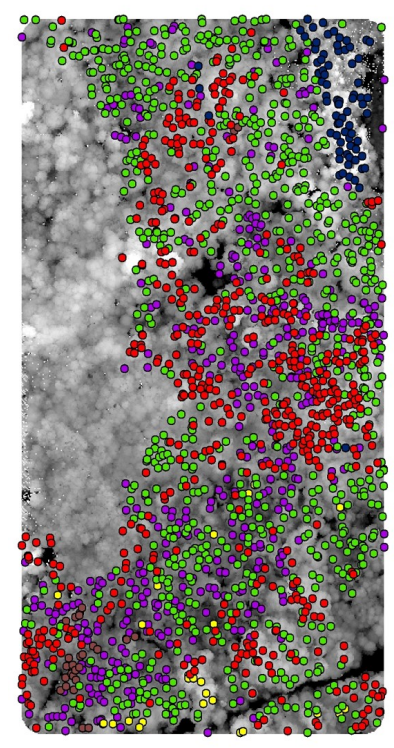

(a) ITC species mapping

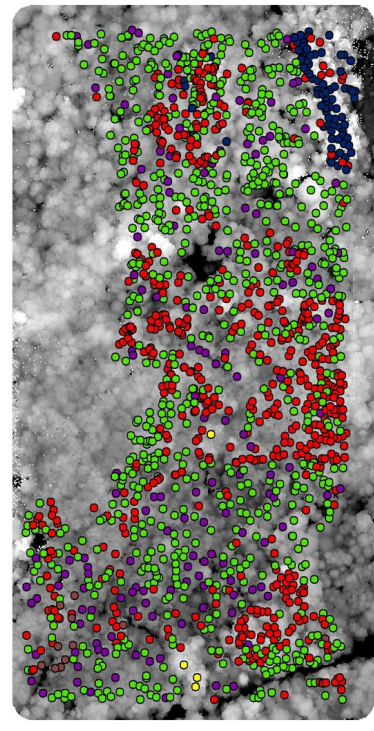

(b) Ground truth

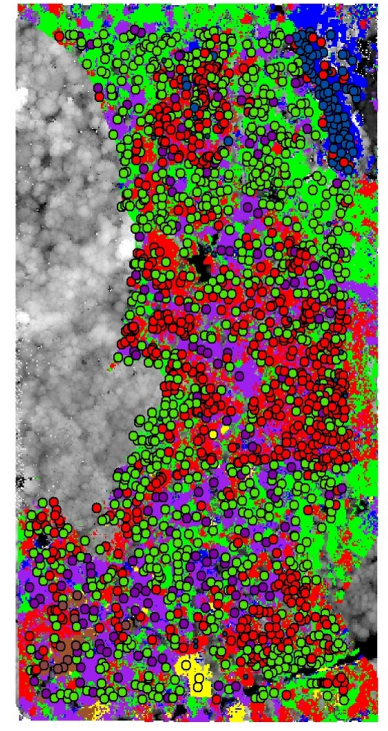

(c) Ground truth with pixel-level species classification

Fig. 1.6 Mapping individual tree species at ITC-level and ground truth. The background images in (a)-(c) are the digital surface model derived from LiDAR data. The coloured map in (c) is pixel-level species classification, where each colour indicates different species. The circles in different colours represents ITCs of different species. More details will be explained in Chapter 4 


\subsubsection{Physiological indices of individual trees in Mediterranean wood- land estimated using fused airborne LiDAR and hyperspectral imagery}

In this section, we modify the workflow developed in the Chapter 4 to estimate physiological indices of species with respect to environmental gradients. The chapter is the outcome of a collaboration with my supervisors - Carola-Bibiane Schönlieb and David Coomes - and their postdoc Xiaohao Cai [170].

The physiological properties of species in forest canopies have important influences on biogeochemical cycles, including energy fluxes and nutrient cycling [11]. Monitoring physiological features of canopies using multi-sensor imaging could help to understand the impacts of environmental and taxonomic diversity on ecosystem functioning [17]. In this study, we characterise foliar pigment contents, water contents and light use efficiency of 90,000 trees in a Mediterranean woodland dominated by two oak species (Quercus suber and Quercus canariensis) and explore variation in those indices along gradients of elevation, aspect and slope. The modified workflow includes six steps. The first step includes registration of hyperspectral imagery and LiDAR data. The second step contains reducing dimensions of hyperspectral imagery using robust PCA [53]. In the third step, ITCs are delineated. In the fourth step, hyperspectral imagery is filtered out using ITCs, NDVI and hillshade information. The fifth step is about species identification. Finally, five physiological indices (PRI, RGRI, CRI, ARI and WBI) $[102,104,111,112,215]$ are extracted at ITC level and analysed along with environmental gradients. No attempt is made to use radiative transfer models to separate leaf-level signals from soil and canopy effects, although we recognise that this might be an important additional step.

Figure 1.7 shows the PRI variations of $Q$. canariensis and $Q$. suber with altitude. The analysis shows that PRI decreases with an increase in altitude. Approximately $20 \%$ of PRI are influenced by altitudinal gradients. Our workflow also indicates that $Q$. canariensis 


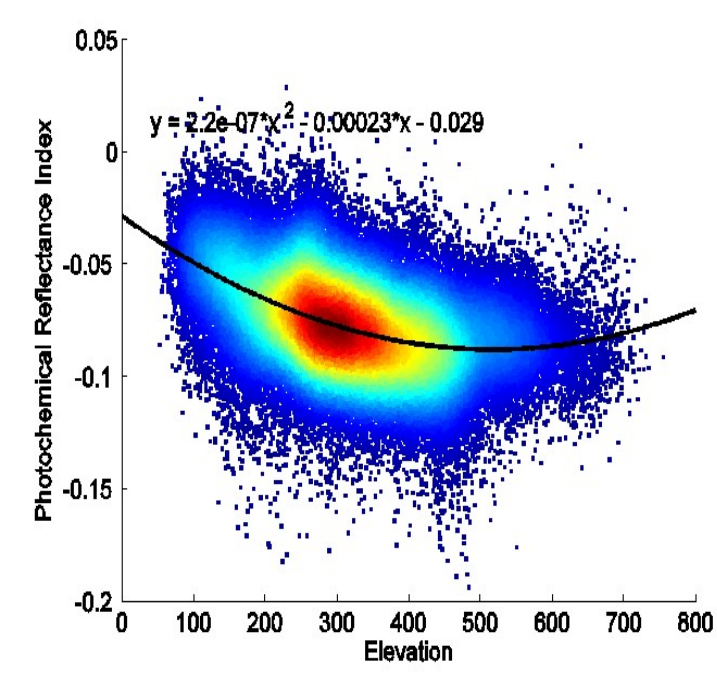

(a) PRI variations of $Q$. suber

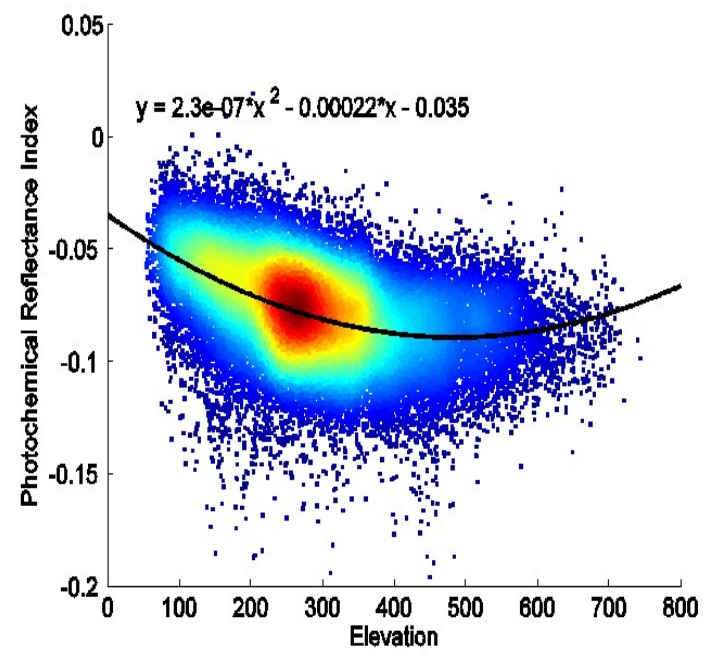

(b) PRI variations of $Q$. canariensis

Fig. 1.7 Variation in PRI obtained from the hyperspectral imagery of (a) 59962 Q suber and (b) $31784 Q$. canariensis trees with respect to altitude. Black line indicates the curve fitted by regression. Colour represent density of observations scaled from blue to red, i.e. red indicates high frequency of observations, and blue means low density of observations fitted using the dscatter function in MATLAB.

is more sensitive to altitudinal gradients than $Q$. suber. The results of our analysis are in agreement with previous studies on physiological responses of Mediterranean canopy species to altitude $[227,226]$. In addition, canopy water contents of $Q$. canariensis decreased as elevation goes up. This result was also in good agreement with the ecological measurements of Q. canariensis [107, 252].

\subsubsection{Organisation of the thesis}

This thesis develops a series of technical solutions for ecological application of multi-sensor imagery. It focuses on ways to fuse LiDAR and hyperspectral imagery, and applications of fused datasets. First, technical difficulties related with airborne multi-sensor imaging described in Section 1.5 are addressed. Secondly, multi-sensor imaging is applied to the mapping of species and the monitoring of canopy species physiology. The performance 
of our new approach is demonstrated with airborne remote sensing data obtained from a temperate deciduous forest and a Mediterranean woodland:

- Co-align multi-sensor airborne remote sensing: LiDAR, hyperspectral and aerial photographs in a wooded landscape (Chapter 2)

- Develop a 3D individual tree crown delineation algorithm using LiDAR and hyperspectral imagery (Chapter 3)

- Design a novel protocol of airborne multi-sensor remote sensing to map species in both pixel and ITC levels (Chapter 4)

- Develop a workflow for multi-sensor airborne remote sensing to monitor plant physiological responses with respect to environmental gradients at ITC level (Chapter 5) 



\title{
Chapter 2
}

\section{Non-parametric image registration of}

\author{
airborne LiDAR, hyperspectral and
}

photographic imagery of wooded

landscapes

\subsection{Introduction}

Airborne multi-sensor imaging is increasingly used to examine vegetation properties $[33,47]$. The advantage of using multiple types of sensors is that each detects a different feature of the vegetation, so that collectively they provide a detailed understanding of the ecological processes $[15,74,134]$. Specifically, Light Detection And Ranging (LiDAR) devices produce detailed point clouds of where laser pulses have been backscattered from surfaces, giving information on vegetation structure $[174,181]$; hyperspectral sensors measure reflectances within narrow wavebands, providing spectrally detailed information about the optical properties of targets $[15,12]$; while aerial photographs provide high spatial-resolution imagery so 
Non-parametric image registration of airborne LiDAR, hyperspectral and photographic imagery of wooded landscapes

that they can provide more feature details which cannot be identified from hyperspectral or LiDAR intensity images $[135,201]$. Using a combination of these sensors, individual trees in forests can be mapped, enabling invasive species to be monitored $[12,16]$, carbon storage to be assessed [9] and leaf physiological processes to be inferred [15, 19]. Accurate alignment of images is critical for the success of the aforementioned applications. However, images taken from different sensors or angles have relative rotation, translation or scale mismatches, and rugged terrain can cause complex displacement between images [271, 164]. As a result, aligning images is challenging.

Image registration involves transforming a template image $T$ using a transformation $\varphi$ so that it aligns with a reference image $R$. Alignment of remotely sensed images (known as image registration) is currently conducted with feature-based methods [33, 164, 44, 177, 263, 182, 178, 114, 258, 100, 150, 115, 262], intensity-based methods [271, 58, 59, $247,209,118,157,101,131]$ or a combination of the two $[164,180,265]$. Feature-based methods rely on identifying common features in $R$ and $T$. Using these common features, transformation parameters of feature-based methods are calculated such that the location of the features in the transformed template image $T_{r e g}$ are identical to those in $R$. Features in common between the reference and template images (i.e. points, patches or edges in the images), can be obtained by manual selection $[164,44]$, or by a variety of automatic techniques including edge detection $[33,177,263]$, scale invariant feature transformation (SIFT) [182, 178, 114, 258, 265], speeded-up robust features detection (SURF) [30], random sample consensus $[100,150]$, feature segmentation $[114,115]$ or phase congruency methods [262]. The performance of these approaches at detecting common features depends on image quality and it can be difficult to locate corresponding features between images when datasets have different spatial resolutions or optical properties $[58,59]$. Furthermore, in the case of multi-sensor imaging, some features in the reference image may not be present in the template image, or vice versa. In contrast, intensity-based methods involve maximising the 
similarity in intensity values between the transformed template image $T_{\text {reg }}$ and $R[271,58,59$, 247, 209]. The most often used similarity measures are Normalised Cross Correlation (NCC) [101, 131], Mutual Information (MI) [59, 70, 247, 209] and Normalised Gradient Fields (NGF) $[118,157]$. In theory, intensity-based methods are fully automatic, but in practice they are often mathematically ill-posed, in the sense that the registration solution might not be unique and a small change within the data might result in large variation in registration results [120]. In addition, different types of sensors affect the similarity between images significantly, therefore the choice of similarity measure for the intensity-based methods is very important $[47,59,247,209,180,265,70,141,50]$.

According to the form of the transformation $\varphi$ used in image registration methods, registration methods can also be classified as parametric or non-parametric (NP). Parametric registration methods use a set of parameters to construct $\varphi$; these parameters may, for example, be used to explicitly generate translation and rotation, or an affine transformation (i.e. one that preserves points, straight lines and planes) [48]. In contrast, in a NP registration method the transformation $\varphi$ is no longer parametrised, compare Section 2.3 for details.

This paper develops a NP registration method - which we refer to as NGF-Curv - based on variational formulation as an alternative to the well-established feature-based and intensitybased approaches [164]. NP registration methods are already well-established in mathematical analysis, medical imaging communities and computer vision [271, 194, 243, 176, 51, 62 , $32,254,98]$ but have yet to permeate far in the field of remote sensing. NP registration methods are based on a variational formulation within which a cost function is minimised. They have been developed to overcome the ill-posedness of established methods by considering not only the similarity between images but also the regularity of the transformation in the calculated cost function. These methods are not mentioned in recent reviews of registration methods in remote sensing $[164,80]$, and to the best of our knowledge, have never been used in this field. We demonstrate how NP registration can be used to register three types of air- 
Non-parametric image registration of airborne LiDAR, hyperspectral and photographic imagery of wooded landscapes

borne remote sensing data sampled over wooded landscapes (i.e. LiDAR, hyperspectral and photographic imagery). The benefits of the NP registration method are illustrated, focussing particularly on its strong performance regardless of the number of different data types or degree of preprocessing. The datasets used to exemplify the approach are introduced in Section 2.2. Then in Section 2.3, the mathematical concepts of the NP image registration algorithm based on variational formulation are introduced. The demonstration of the effectiveness of the approach is given in Sections 2.4 and 2.5. Finally, Section 2.6 explores the behaviour of alternative similarity measures and gives recommendations for future work.

\subsection{Data}

This section briefly addresses the methodologies and properties of the datasets used for registration in this paper. Acquisition of remote sensing datasets was conducted in three areas of the Los Alcornocales Natural Park, Spain (lat $36^{\circ} 19^{\prime}$, long $5^{\circ} 37^{\prime}$ ) on 10 April 2011, by Airborne Research and Survey Facility of the UK's Natural Environment Research Council (NERC-ARSF) and preprocessed by their Data Analysis Node [203]. The study area is a typical Mediterranean wooded landscape, where grassy fields in the valleys are surrounded by open woodland on hills, and rocky outcrops at higher altitude. The study area was dominant by Quercus suber and Quercus canariensis. The estimated tree cover area is $52 \%$ of the LiDAR survey area. The airplane flew at a nominal height above ground of approximate $3000 \mathrm{~m}$ and was equipped with LiDAR and hyperspectral imagers, as well as a digital camera. LiDAR [Leica ALS 50-II] emits pulses of monochromatic laser light (1064 nm) to scan topographical and geometrical structures of the surface, creating three-dimensional point clouds representing the points at which pulses are backscattered off surfaces and returned to the aircraft. The field of view was $12^{\circ}$. Each point has an associated intensity value, which correlates with the proportion of a pulse's energy which is returned to the sensor. However, the radiometric properties of LiDAR intensity are not completely known - LiDAR 
pulse intensity values are controlled by an automatic gain control (AGC) system during the acquisition process, so the intensity of the return is a function of unknown varying pulse energy as well as the backscattering properties of the ground surface $[149,156,155]$. NERCARSF preprocessed these LiDAR data and georeferenced them to the Universal Transverse Mercator (UTM) projection with WGS-84 datum [203]. The estimated georeferencing error of the LiDAR data is around $0.15 \mathrm{~m}$ horizontally and $0.30 \mathrm{~m}$ vertically. The average LiDAR point density over the study site was 2 points per square metre $\left(\mathrm{m}^{2}\right)$. In order to compare LiDAR imagery with other datasets in our experiment, LiDAR point clouds were projected onto a two-dimensional image plane by ignoring the height information for each LiDAR point. LiDAR intensity was calculated in $1 \mathrm{~m}$ pixels as the average of the all-return pulse intensities, with preprocessing by focal statistics function in ArcGIS software and image contrast enhancement by MATLAB build-in histogram equalisation function histeq. Using first returns yielded qualitatively similar results to using all returns.

Hyperspectral imaging spectrometers measure solar energy reflected off the earth's surface within a swath of land. Hyperspectral data were gathered using the AISA Eagle (Specim Ltd., Finland), which are pushbroom sensors with 255 spectral bands covering 400-970 nm wavelengths across $2300 \mathrm{~m}$ swath width with $3 \mathrm{~m}$ spatial resolution. The hyperspectral sensors record reflected energy in digital numbers, which were converted to spectral radiance $\left(\mu \mathrm{Wcm}^{-2} \mathrm{sr}^{-1} \mathrm{~nm}^{-1}\right)$ and then provided to us. Before image registration, hyperspectral imagery was atmospherically corrected using ATCOR-4 (Rese Ltd., Switzerland), which converts radiance values to reflectances. An accurate navigation system integrated with boresight calibrated hyperspectral sensors provides geocoordinates of each pixel in the hyperspectral imagery, which meant that the hyperspectral images could be orthorectified by digital elevation models (DEM) from ASTER and LiDAR data and then georeferenced to the UTM projection with WGS-84 datum. The estimated georeferencing error of the hyperspectral image is about 5-10 m horizontally. However, it deteriorates at the edge of the 
Non-parametric image registration of airborne LiDAR, hyperspectral and photographic 36 imagery of wooded landscapes

field of view of the hyperspectral sensors. The spectral bands for a given scan line were all recorded simultaneously. True colour composite of RGB bands (640, 549 and $460 \mathrm{~nm}$ ) were converted to grey images by MATLAB build-in function rgb2gray and the grey image was used for registration purposes.

Aerial photographs were acquired during the flight using a Leica RCD-105 Digital Frame Camera. Each photograph has $7212 \times 5408$ pixels. Since the spatial resolution of aerial photographs is much higher than that of hyperspectral images, aerial photos can help to identify objects more accurately. However, aerial photographs were not integrated with the aircraft navigation system, so they were not orthorectified or georeferenced during preprocessing. Metadata associated with aerial photographs informs of the time, location and altitude of aircraft when each photo was taken. We assumed that the location was the centre of each image and that the spatial resolution of each pixel equaled to $0.3 \mathrm{~m}$.

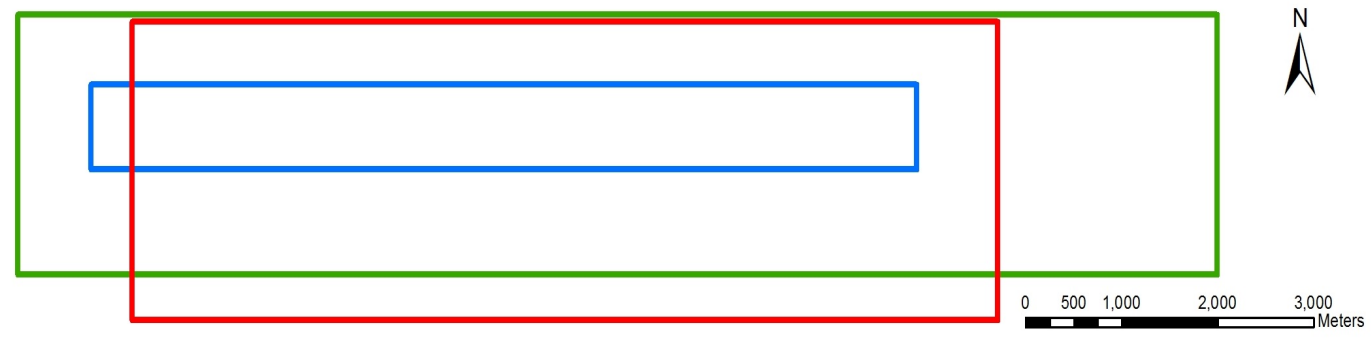

Fig. 2.1 The aerial coverages of LiDAR (blue line), hyperspectral images (green line) and aerial photographs (red line) recorded in line 2 of the NERC-ARSF survey of Spanish woodland. The LiDAR aerial coverage is about $6.4 \mathrm{~km}^{2}$ with $1 \mathrm{~m}$ spatial resolution, while the hyperspectral imagery and aerial photographs are $28.4 \mathrm{~km}^{2}$ and $23.4 \mathrm{~km}^{2}$ respectively. The spatial resolutions of hyperspectral imagery and aerial photos are $3 \mathrm{~m}$ and $0.3 \mathrm{~m}$.

The aerial coverages and resolutions of LiDAR, hyperspectral images and aerial photographs are given in Figure 2.1. We can see that LiDAR imagery in blue line in Figure 2.1 has a narrower width and smaller aerial coverage than the hyperspectral imagery (green) and aerial photos (red). Our registration scheme aligns the hyperspectral imagery and aerial 
photos onto the LiDAR image because combining three different types of sensors is the main purpose of this research.

If the preprocessed data had been georeferenced then the images could have been aligned using the georeference coordinates. However, uncertainties from sensor distortions and boresight errors often cause mis-alignment, so image registration techniques need to be applied in order to precisely align images. Registration of aerial photos onto hyperspectral images or LiDAR imagery is even more challenging because they were neither orthorectified nor georeferenced when delivered. This paper provides a robust and accurate approach for registering all three datasets.

\subsection{Method}

This section will briefly describe the mathematical concept of NP image registration, and the particular registration method NGF-Curv that we use for the registration of images in our dataset (see [99] for further details). Let $R$ and $T$ be the given reference and template images, respectively, modelled as functions defined on a finite two-dimensional grid $\Omega$ and mapping a point $x$ on the grid to a real intensity value $R(x)$ and $T(x)$, respectively. The resolutions of $R$ and $T$ do not necessarily have to be the same, that is they can have different sizes in vertical and horizontal directions. In this case, spline interpolation scheme was used to rescale them to the same grid $\Omega$.

When registering the template $T$ with the reference image $R$, we find a suitable transformation which maps $T$ to $R$ such that the transformed version of $T$ is similar to $R$. This transformation maps the grid of $T$ to the grid of $R$. A generic variational method for finding this transformation is as a solution $\varphi: \Omega \rightarrow \Omega$ of

$$
\min _{\varphi}\left\{\sum_{x \in \Omega} D[T(\varphi(x)), R(x)]+\alpha S(\varphi)\right\}
$$


Non-parametric image registration of airborne LiDAR, hyperspectral and photographic imagery of wooded landscapes

where $D$ is a similarity measure that quantifies the difference between the distorted template $T$ and reference image $R, S$ is a so-called regularisation term that imposes appropriate regularity on the transformation $\varphi$ and $\alpha$ is a positive parameter that balances the importance of the similarity measure against the regularisation term. Solutions of (2.1) for the registration problem are given by $[194,193,98]$. In the particular case of NP registration considered in this paper, the transformation function $\varphi$ can be expressed as the sum of identity and displacement $u$, which is

$$
\varphi: x \rightarrow x-u(x)
$$

A standard choice for $D$ in $(2.1)$ is

$$
D[T(\varphi(x)), R(x)]=\frac{1}{2}[T(x-u)-R(x)]^{2},
$$

but this has the disadvantage of not being contrast-invariant [194]. This can be corrected by other kinds of distance measures, for example NCC (Normalised Cross Correlation), MI (Mutual Information) and NGF (Normalised Gradient Field). The NCC distance measure is defined as:

$$
D^{\mathrm{NCC}}\left[T_{\text {reg }}, R\right]=1-\frac{\left(\operatorname{vec}\left(T_{\text {reg }}\right)^{T} \operatorname{vec}(R)\right)^{2}}{\left(\operatorname{vec}\left(T_{\text {reg }}\right)^{T} \operatorname{vec}\left(T_{\text {reg }}\right)\right)\left(\operatorname{vec}(R)^{T} \operatorname{vec}(R)\right)},
$$

where $T_{\text {reg }}$ denote the registered template after transformation to the reference image (i.e. $=$ $T(\varphi)$ ), and $\operatorname{vec}(A)$ generates a vector by concatenating the columns of the tensor $A$. This distance measures the normalized cross-correlation between $T_{r e g}$ and $R$. The MI distance 
measure is defined as:

$$
\begin{aligned}
D^{\mathrm{MI}}\left[T_{\text {reg }}, R\right]= & \sum_{x \in \Omega} \rho_{T_{\text {reg }}}(x) \log \rho_{T_{\text {reg }}}(x)+\sum_{y \in \Omega} \rho_{R}(y) \log \rho_{R}(y) \\
& +\sum_{x \in \Omega} \sum_{y \in \Omega} \rho_{\left[T_{\text {reg }}, R\right]}(x, y) \log \rho_{\left[T_{\text {reg }}, R\right]}(x, y)
\end{aligned}
$$

where $\rho_{T_{\text {reg }}}$ and $\rho_{R}$ are the marginal densities, and $\rho_{\left[T_{\text {reg }}, R\right]}$ is the joint density, see [194] for more details. In this paper we use the NGF similarity measure $[118,194]$. Here, the normalized gradient $\frac{\nabla I}{|\nabla I|}$ of an image $I$ is used to measure similarity between $R$ and $T$. More precisely, the NGF measure is defined as:

$$
\operatorname{NGF}(I, \eta)=\operatorname{vec}\left(\frac{\nabla I}{\sqrt{|\nabla I|^{2}+\eta^{2}}}\right)
$$

where $\eta>0$ is an edge parameter. The edge parameter models the level of the noise present in $I$ such that image values below this parameter are ignored. Then the NGF distance measure is defined as:

$$
D^{\mathrm{NGF}}\left[T_{r e g}, R\right]=1-\left(\left(\operatorname{NGF}\left(T_{r e g}, \eta\right)\right)^{T} \operatorname{NGF}(R, \eta)\right)^{2}
$$

which, if minimised, maximises the linear dependency (alignment) of the NGF of $T$ and $R$.

The regularisation term $S$ encodes the regularity that should be imposed on the transformation $\varphi$ to reduce the ill-posedness of the registration problem. For an overview of different regularisation terms and their effect on the registration, see [194, 193]. In what follows we use a curvature regularisation $[118,98]$, which is

$$
S^{\text {curv }}(\varphi)=S^{\text {curv }}(u)=\frac{1}{2} \sum_{x \in \Omega}|\triangle u(x)|^{2} .
$$


Non-parametric image registration of airborne LiDAR, hyperspectral and photographic imagery of wooded landscapes

This regularisation results in the registration accuracy being dependent on the smoothness of the displacement $u$ between $R$ and $T$ [193]. In particular, curvature regularisation penalises oscillations in $u$ since it can be regarded as an approximation of the curvature of $u$ [193]. One advantage of curvature regularisation is that it does not require affine preregistration steps. Other regularisation techniques, such as fluid registration $[65,43]$, are sensitive to affine linear displacement such that preregistration with affine linear transformation is required, see $[193,99,98]$.

The method developed in this paper is based on minimising the following function:

$$
J(u)=\sum_{x \in \Omega} D^{\mathrm{NGF}}\left[T_{r e g}(x), R(x)\right]+\frac{\alpha}{2} \sum_{x \in \Omega}|\triangle u(x)|^{2},
$$

where $D^{\mathrm{NGF}}$ is the NGF similarity measure defined in (2.6) and $S^{\text {curv }}$ is the curvature regularisation term defined in (2.7). In what follows, we refer to this method as the NGF-Curv method (NGF-Curv for short).

For the numerical minimisation of (NGF-Curv) we use the Image Registration software package (FAIR) ${ }^{1}$. There, the minimiser of (NGF-Curv) is computed iteratively via a semiimplicit scheme for the so-called Euler-Lagrange equation for (NGF-Curv). The latter is the equation that arises as the spatially discrete version of the Gâteaux derivative of the continuous functional $J$, which reads [98]:

$$
f(x, u(x))+\alpha \triangle^{2} u(x)=0 \quad \text { for } x \in \Omega,
$$

where $f(x, u(x))$ is the discretisation of the derivative of the distance measure $D$. In order to solve equation (2.8) a semi-implicit iterative scheme is used which introduces an artificial

\footnotetext{
${ }^{1}$ MATLAB version of FAIR http://www.siam.org/books/fa06/
} 
time step $\Delta t$ and computes the fixed point of the equation [194, 193, 98]:

$$
u^{k+1}(x)-\Delta t \alpha \Delta^{2} u^{k+1}(x)=u^{k}(x)+\Delta t f\left(x, u^{k}(x)\right)
$$

where $u^{k}(x)$ denotes the $k$-th iterate of the scheme. Further details regarding discretisation and numerical optimisation are provided in [194]. Since remote sensing datasets contain largescale surface information, it is computationally expensive to conduct entire image registration steps at the original resolution [194]. FAIR provides a multilevel image-registration scheme, producing a series of images varying in resolution, such that registration results from a coarser image can be used to initialise the registration on finer resolutions of the images. The multilevel scheme reduces the amount of expensive computation and the chance of being trapped in local minima during the iterative search, as images are much smoother in coarse resolution [194, 119].

\subsection{Application of the registration approach to the airborne remote sensing dataset}

NGF-Curv requires that images are identical in size, so the first step of the process was to match the geographical boundaries of all datasets to each other, by reducing the number of features present in either $R$ or $T$. Since both hyperspectral and LiDAR intensity images contain geo-coordinates, geographical boundary matching of them is straightforward. But the aerial photographs were neither georeferenced nor orthorectified and matching the boundary between aerial photographs and other datasets was therefore challenging. For the latter we used the geo-coordinate at which each photo was taken as the centre of each aerial photograph. Then the geographic boundary of each aerial photo was roughly calculated by counting the approximate number of pixels of an aerial photograph and adding $300 \mathrm{~m}$ in $x$ 
Non-parametric image registration of airborne LiDAR, hyperspectral and photographic imagery of wooded landscapes

and $y$ directions to compensate the errors caused by rough approximation. Hence the size of each aerial photograph image was assumed to be:

$$
L_{r_{x}}=0.3 L_{t_{x}}+300, \quad L_{r_{y}}=0.3 L_{t_{y}}+300,
$$

where $L_{t_{x}}$ and $L_{t y}$ are the number of pixels of aerial photographs in $x$ and $y$ directions, and the 0.3 multiplier converts pixels into metres. Approximately, $L_{t_{x}}=5000$ and $L_{t_{y}}=7000$ for the photos used in this paper.

LiDAR intensity data were used alongside a grey image converted from a true colour composite $(640,549$ and $460 \mathrm{~nm})$ of the hyperspectral images using the MATLAB build-in function rgb2gray. Although it would seem natural to use the band at $1065 \mathrm{~nm}$ wavelength of the hyperspectral imagery - which corresponds to the LiDAR intensity wavelength - this band suffers from low signal-to-noise ratio, so was not used.

NP image registration with a variational formulation finds the optimised location for each pixel by comparing gradients of two images. This is achieved by numerical optimisation methods. The FAIR toolbox provides three different second-order optimisation schemes: Gauss-Newton, 1-BFGS and Trust region. The 1-BFGS optimisation scheme was adopted in this paper. The choice of optimiser can influence the performance of image registration, but evaluating their performances was beyond the scope of this paper. NP registration yielded optimised spatial coordinates of each pixel, which were used for the transformation of original hyperspectral images. During the transformation, the hyperspectral images were resampled and regridded by a nearest neighbour method. Nearest neighbour estimates were chosen from existing values rather than averaging neighbouring pixels, thus minimising artifacts. This is important because hyperspectral imagery should preserve physically meaningful values.

Choosing optimal parameters in (NGF-Curv) is an important step of the registration process, but these are difficult to find automatically (although see [118, 5] for examples of automatic edge parameter $\eta$ selection once noise level and image volume are known). 
We used a trial-and-error approach to find $\eta$ and smoothness parameter $\alpha$, which was time consuming. Fortunately, tuning of parameters for each registration of remote sensing images is not normally required - a single calibration for template and reference images taken by two different sensors was enough to obtain reasonable results in most cases. For the registration of a hyperspectral image onto a LiDAR intensity image the optimal values of $\alpha$ and $\eta$ were found to be 5000 and 0.1 , respectively.

The aerial photo was aligned with the hyperspectral image because the swath widths of the hyperspectral images $(2100 \mathrm{~m})$ and aerial photos $(2400 \mathrm{~m})$ were similar, while the LiDAR images were much narrower $(800 \mathrm{~m}$ ) (see Figure 2.1). The narrow swath width of the LiDAR makes it difficult to find a region of interest in the aerial photo corresponding to that in the LiDAR as the geographic boundary of the aerial photo is roughly approximated, because of the roll, pitch, yaw of aircraft and local topographic distortion. The registration of the aerial photographs onto the hyperspectral images is challenging because aerial photographs are distorted by various effects, including topography, lense distortion and viewing angle. As we assumed the location where each aerial photo was taken as the centre of the image, corresponding hyperspectral images of size $L_{r_{x}} \times L_{r_{y}} \mathrm{~m}^{2}$ were extracted from the hyperspectral imagery and used as the reference image. Curvature registration with NGF distance measure (NGF-Curv) was employed to register aerial photographs onto hyperspectral images. Regularisation parameter was set to $\alpha=2.5 \times 10^{5}$ and the edge parameter $\eta=0.03$. RGB bands of hyperspectral images and RGB aerial photos were both transformed to grey intensity images before registering them to each other to increase the processing speed and the robustness of the registration. After the registration of the aerial photographs onto the hyperspectral imagery, a mosaic of the aerial photos was created which was then aligned with the LiDAR data by applying the already computed LiDAR-hyperspectral imagery transformation.

Numerical experiments were conducted to compare the NGF-Curv method with wellknown parametric registration methods based on alternative distance measures (i.e. NCC, MI 
Non-parametric image registration of airborne LiDAR, hyperspectral and photographic imagery of wooded landscapes and NGF) and the feature-based parametric method SURF [30]. These established methods are computationally efficient and widely used (see Sections 2.1 and 2.3 for references); SURF in particular is recognised as a robust local feature detector that is effective at featurebased parametric registration. Approaches were compared in terms of the mean, standard deviation (std), minimum (min) and maximum (max) of $\left|T_{\text {reg }}-R\right|$, and the correlation (corr) between $T_{r e g}$ and $R$, using MATLAB build-in commands mean, std, min, max and corrcoef, respectively. Mean and std reflect the distance between $T_{r e g}$ and $R$, so smaller magnitudes indicate better registration; min and max give the range of the differences between $T_{\text {reg }}$ and $R$, while corr represents the linear dependence of $T_{\text {reg }}$ and $R$ (larger values indicate better registration). To compare computation efficiency, CPU time in seconds were given. All analyses were conducted using Intel Xeon E5-2630 2.30GHz PC. Numerical experiments were conducted in LiDAR-hyperspectral registration and two different scenarios of aerial photograph-hyperspectral imagery registration. The reason why we give two examples of the aerial photograph-hyperspectral imagery registration is that topographical distortion of aerial photograph in rugged terrain may be larger than that in flat terrain.

\subsection{Results}

Comparison of NGF-Curv with established approaches (Table 2.1) show mean, standard deviation, maximum, minimum, correlation and computational time. The mean, standard deviation and correlation in Table 2.1 support NGF-Curv performed better than NCC, MI, NGF and SURF in all cases, while minimum and maximum errors show no difference. NGFCurv requires more computational time than other methods in all examples. This is because NGF-Curv computes an optimal transformation of each pixel, while parametric methods find a set of transformation parameters. Therefore, the degree of freedom of NGF-Curv is much larger than that of NCC, MI, NGF and SURF methods, thus slowing time. We now consider these results in detail. 
Table 2.1 Mean, standard deviation, minimum and maximum of $\left|T_{\text {reg }}-R\right|$, correlation between $T_{\text {reg }}$ and $R$, and CPU time in seconds of each method

\begin{tabular}{c|c||c|c|c|c|c|c}
\hline \multicolumn{2}{c|}{} & $T$ & NCC & MI & NGF & SURF & NGF-Curv \\
\hline \hline \multirow{4}{*}{ Fig. 2.2 } & mean & 73.70 & 66.89 & 73.08 & 72.63 & 74.56 & 65.68 \\
\cline { 2 - 8 } & std & 42.66 & 38.49 & 41.82 & 41.71 & 44.13 & 38.19 \\
\cline { 2 - 8 } & min & 0 & 0 & 0 & 0 & 0 & 0 \\
\cline { 2 - 8 } & max & 255 & 255 & 254 & 255 & 255 & 255 \\
\cline { 2 - 8 } & corr & 0.55 & 0.68 & 0.58 & 0.58 & 0.53 & 0.71 \\
\cline { 2 - 8 } & time & - & 4.48 & 6.63 & 6.96 & 5.86 & 34.85 \\
\hline \hline \multirow{4}{*}{ Fig. 2.3 } & mean & 52.94 & 48.66 & 48.73 & 48.92 & 48.67 & 47.09 \\
\cline { 2 - 8 } & std & 30.88 & 27.90 & 25.21 & 25.46 & 25.12 & 21.39 \\
\cline { 2 - 8 } & min & 0 & 0 & 0 & 0 & 0 & 0 \\
\cline { 2 - 8 } & max & 246 & 249 & 255 & 255 & 255 & 255 \\
\cline { 2 - 8 } & corr & 0.39 & 0.52 & 0.52 & 0.51 & 0.51 & 0.62 \\
\cline { 2 - 7 } & time & - & 10.33 & 11.23 & 15.76 & 163.28 & 380.12 \\
\hline \hline \multirow{4}{*}{ Fig. 2.4 } & mean & 55.61 & 51.37 & 47.09 & 49.01 & 48.61 & 45.23 \\
\cline { 2 - 7 } & std & 39.31 & 37.47 & 31.69 & 34.30 & 33.37 & 26.97 \\
\cline { 2 - 7 } & min & 0 & 0 & 0 & 0 & 0 & 0 \\
\cline { 2 - 7 } & max & 255 & 255 & 255 & 255 & 255 & 255 \\
\cline { 2 - 7 } & corr & 0.18 & 0.28 & 0.35 & 0.32 & 0.32 & 0.55 \\
\cline { 2 - 7 } & time & - & 9.70 & 11.91 & 14.21 & 197.38 & 375.02 \\
\hline
\end{tabular}

The first case we consider is image registration of hyperspectral imagery onto LiDAR (Figure 2.2). As both datasets were georeferenced by the data provider, only small distortions were present (up to $10 \mathrm{~m}$ ) as a result of DEM or navigation inconsistencies [47, 180, 265, 141, 50]. From Figure 2.2 (B1)-(B5), in particular the parts inside the circles marked on the figures $((\mathrm{C} 1)-(\mathrm{C} 5))$ and the quantitative results in terms of mean, standard deviation and correlation in Table 2.1, we see that the results of NCC and NGF-Curv methods are better than the results of MI, NGF and SURF methods at the expense of slow computational time. In this example, the NCC method performed as well as the NGF-Curv method, because both the hyperspectral and LiDAR images were approximately georeferenced before the registration was applied, finding a local minimum was enough to get reasonable outcomes [47]. 


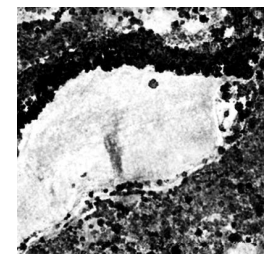

(A1) LiDAR: $R$
Images before registration

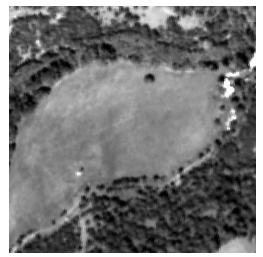

(A2) Hyperspectral (grey): $T$

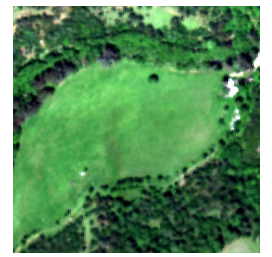

(A3) Hyperspectral (colour): $T$

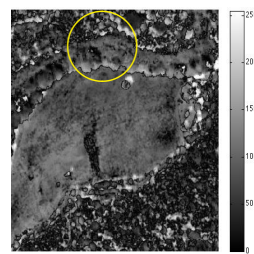

(B0) $|T-R|$

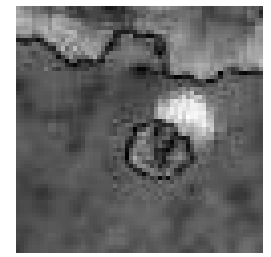

(C0) Highlight (B0)

Hyperspectral image registration on a LiDAR image using five approaches

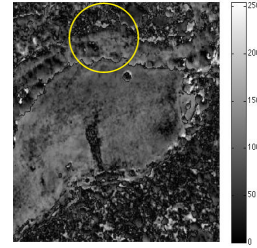

(B1) $\left|T_{r e g}^{\mathrm{NCC}}-R\right|$

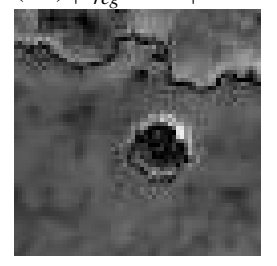

(C1) Highlight (B1)

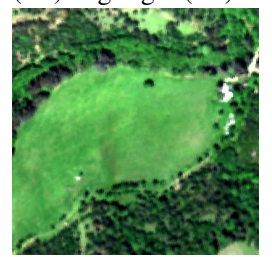

(D1) $T_{r e g}^{\mathrm{NCC}}$

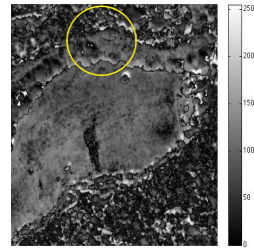

(B2) $\left|T_{r e g}^{\mathrm{MI}}-R\right|$

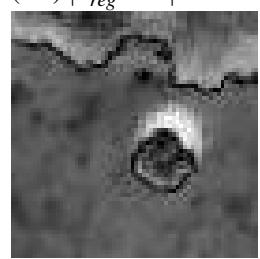

(C2) Highlight (B2)

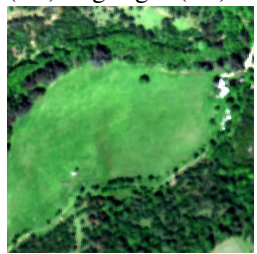

(D2) $T_{r e g}^{\mathrm{MI}}$

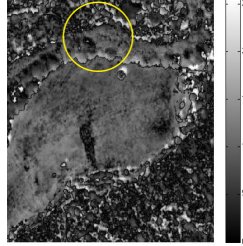

(B3) $\left|T_{r e g}^{\mathrm{NGF}}-R\right|$

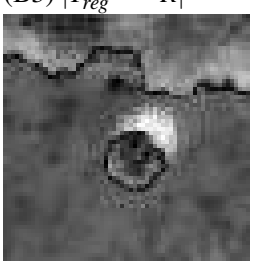

(C3) Highlight (B3)

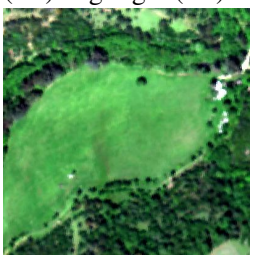

(D3) $T_{r e g}^{\mathrm{NGF}}$

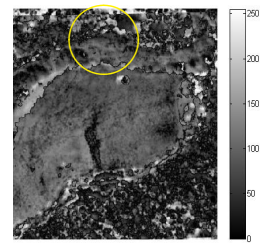

(B4) $\left|T_{r e f}^{\mathrm{SURF}}-R\right|$

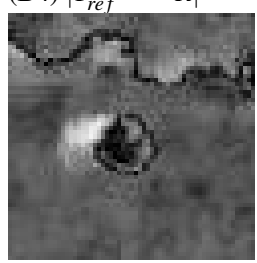

(C4) Highlight (B4)

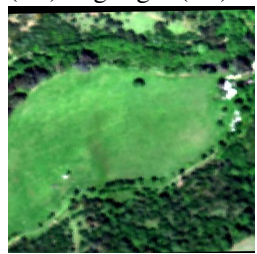

(D4) $T_{r e f}^{\text {SURF }}$

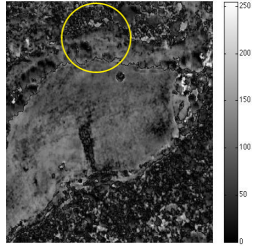

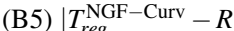

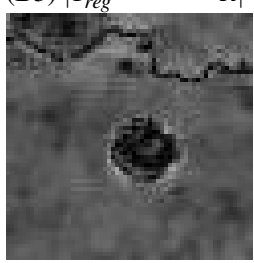

(C5) Highlight (B5)

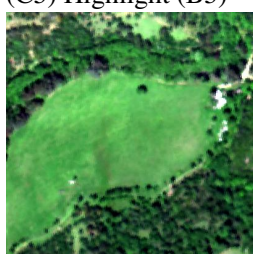

(D5) $T_{r e g}^{\mathrm{NGF}-\mathrm{Curv}}$

Fig. 2.2 Image registration of a hyperspectral image onto a LiDAR intensity image of a Spanish woodland, surveyed by aircraft (scale $400 \times 400 \mathrm{~m}^{2}$ ). The first row shows (A1) a LiDAR intensity reference image $R$; (A2) a hyperspectral template image $T$; (A3) original colour image of (A2); (B0) a map showing the difference between $T$ and $R$ (i.e. $|T-R|$ ), which would be entirely dark if the match was perfect; $(\mathrm{C} 0)$ highlights the circled area of (B0). The second row of maps show $\left|T_{\text {reg }}-R\right|$ obtained by using established methods NCC, MI, NGF, SURF as well as our NGF-Curv method. The results of registration methods are denoted by $T_{r e g}^{\mathrm{NCC}}, T_{r e g}^{\mathrm{MI}}, T_{r e g}^{\mathrm{NGF}}, T_{r e g}^{\mathrm{SURF}}$ and $T_{r e g}^{\mathrm{NGF}-\mathrm{Curv}}$, respectively, in these panels; yellow circle highlights areas of the images where differences among registration methods are seen. The third row zooms in on these highlighted circles. The final row of panels shows the aerial photograph template image after it has been registered using methods (D1) NCC, (D2) MI, (D3) NGF, (D4) SURF and (D5) NGF-Curv. 


\section{Images before registration}

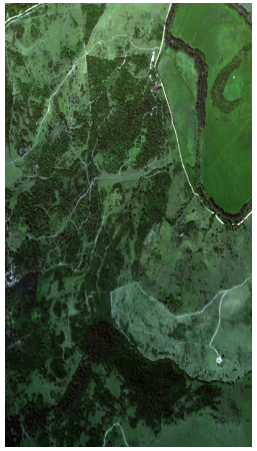

(A1) Hyperspectral: $R$

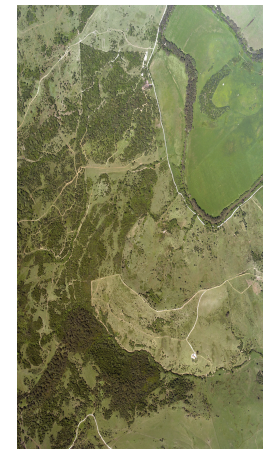

(A2) Photograph: $T$

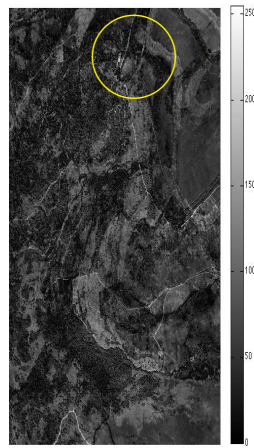

(B0) $|T-R|$

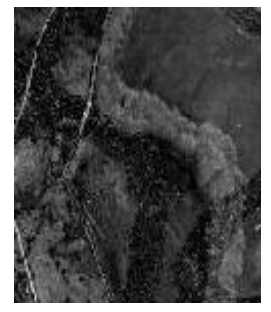

(C0) Highlight (B0)

Aerial photograph registration on a hyperspectral image using five approaches

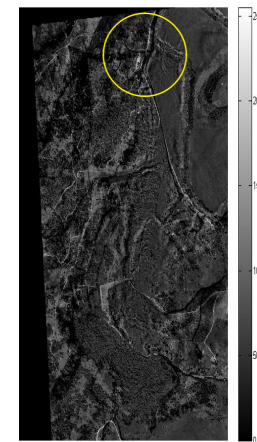

(B1) $\left|T_{\text {reg }}^{\mathrm{NCC}}-R\right|$

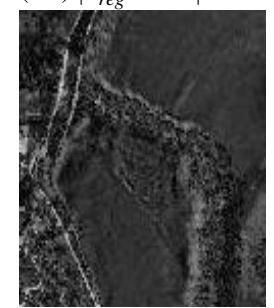

(C1) Highlight (B1)

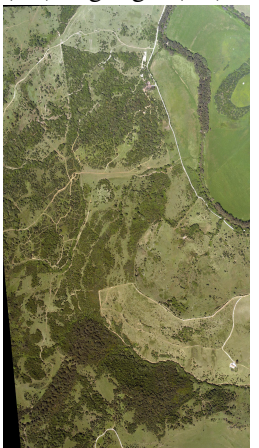

(D1) $T_{\text {reg }}^{\mathrm{NCC}}$

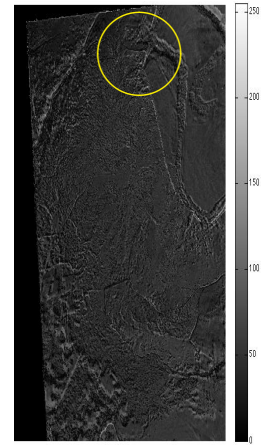

(B2) $\left|T_{r e g}^{\mathrm{MI}}-R\right|$

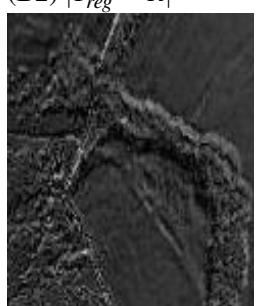

(C2) Highlight (B2)

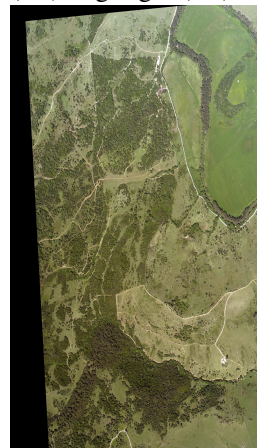

(D2) $T_{r e g}^{\mathrm{MI}}$

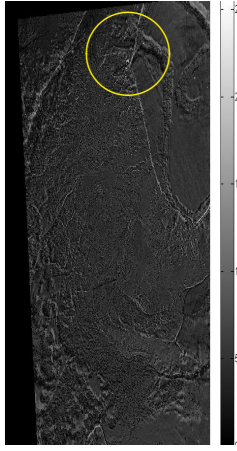

(B3) $\left|T_{\text {NGF }}^{\mathrm{NGF}}-R\right|$

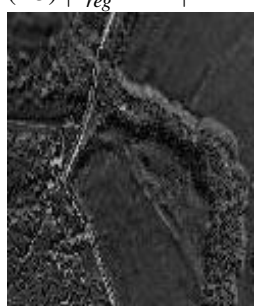

(C3) Highlight (B3)

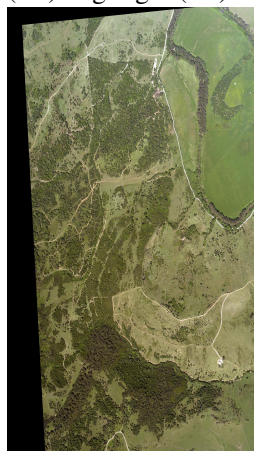

(D3) $T_{\text {reg }}^{\mathrm{NGF}}$

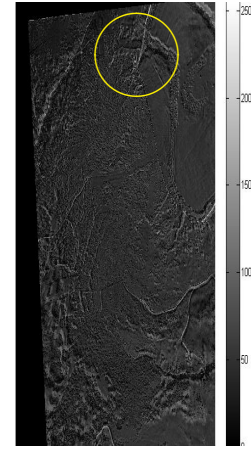

(B4) $\left|T_{\text {ref }}^{\mathrm{SURF}}-R\right|$

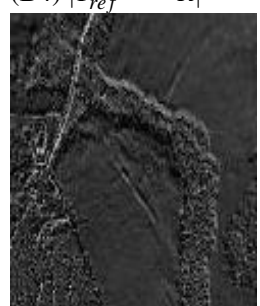

(C4) Highlight (B4)

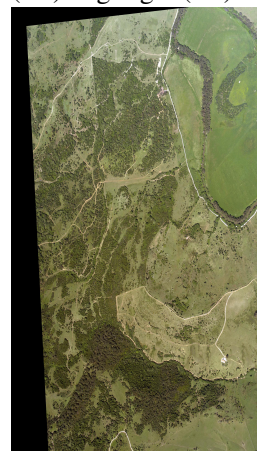

(D4) $T_{r e f}^{\mathrm{SURF}}$

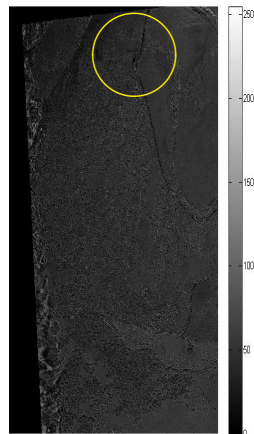

(B5) $\left|T_{\text {reg }}^{\text {NGF-Curv }}-R\right|$

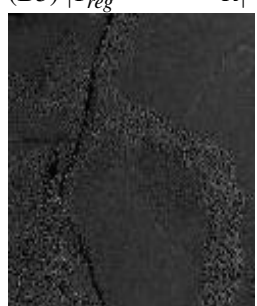

(C5) Highlight (B5)

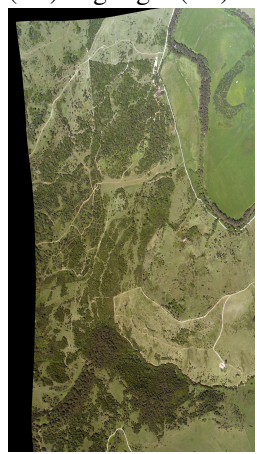

(D5) $T_{\text {reg }}^{\mathrm{NGF}-\mathrm{Curv}}$

Fig. 2.3 Image registration of an aerial photograph onto a hyperspectral image in a region which has flat terrain (scale $2400 \times 1800 \mathrm{~m}^{2}$ ). The first row shows (A1) a hyperspectral reference image $R$; (A2) an aerial photograph template image $T$; (B0) a map showing difference between these images (i.e. $|T-R|$ ); (C0) highlight the circle region of (B0). The second row shows maps of $\left|T_{\text {reg }}-R\right|$ generated by methods NCC, MI, NGF, SURF and our NGF-Curv approach. The third row highlights the circle areas of the maps in the second row. The last row of panels shows the aerial photograph template image after it has been registered using methods (D1) NCC, (D2) MI, (D3) NGF, (D4) SURF and (D5) NGF-Curv. 
Non-parametric image registration of airborne LiDAR, hyperspectral and photographic imagery of wooded landscapes

\section{Images before registration}

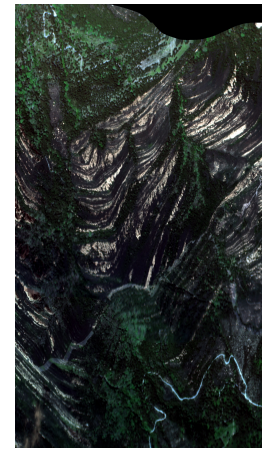

(A1) Hyperspectral: $R$

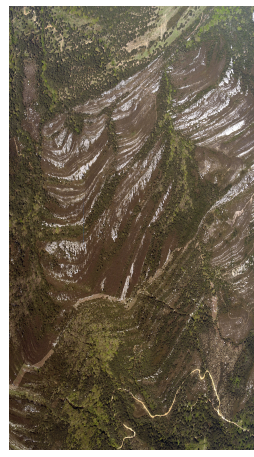

(A2) Photograph: $T$

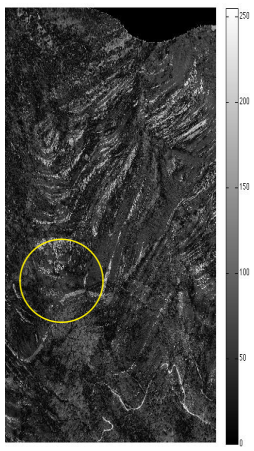

(B0) $|T-R|$

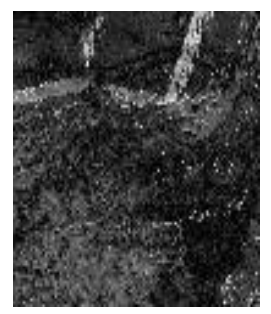

(C0) Highlight (B0)

Aerial photograph registration on a hyperspectral image using five approaches

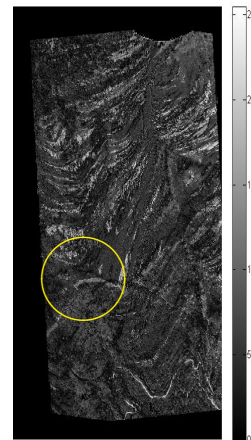

(B1) $\left|T_{r e g}^{\mathrm{NCC}}-R\right|$

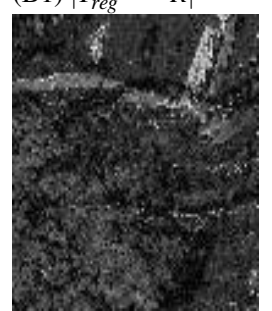

(C1) Highlight (B1)

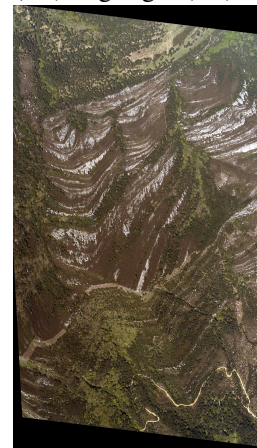

(D1) $T_{\text {reg }}^{\mathrm{NCC}}$

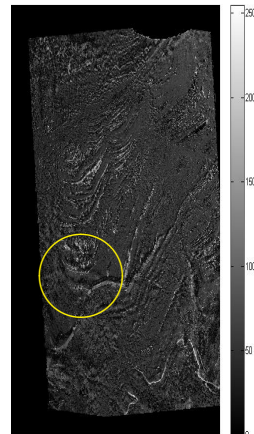

(B2) $\left|T_{\text {reg }}^{\mathrm{MI}}-R\right|$

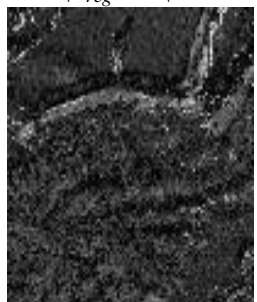

(C2) Highlight (B2)

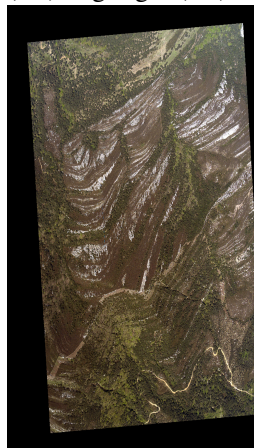

(D2) $T_{\text {reg }}^{\mathrm{MI}}$

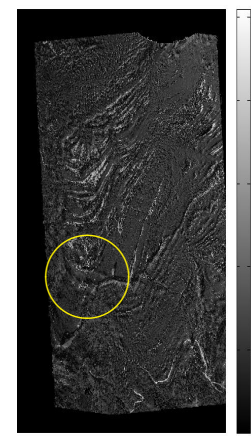

(B3) $\left|T_{r e g}^{\mathrm{NGF}}-R\right|$

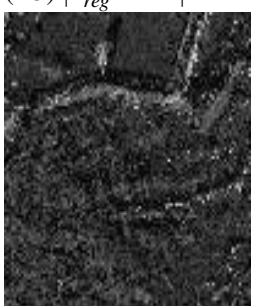

(C3) Highlight (B3)

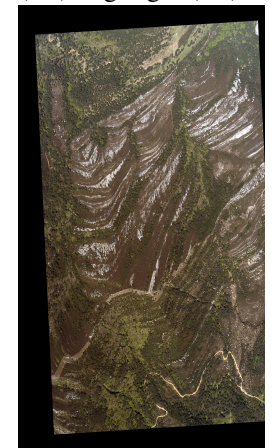

(D3) $T_{\text {reg }}^{\mathrm{NGF}}$

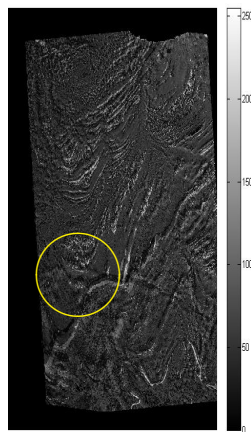

(B4) $\left|T_{r e f}^{\mathrm{SURF}}-R\right|$

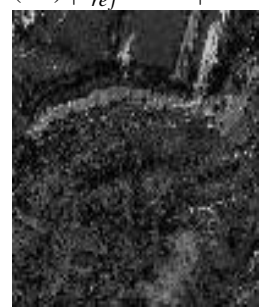

(C4) Highlight (B4)

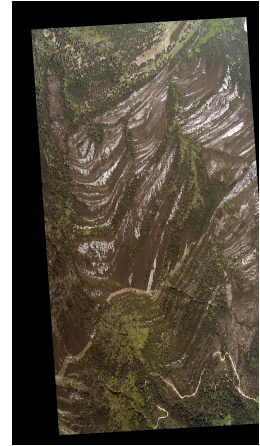

(D4) $T_{r e f}^{\mathrm{SURF}}$

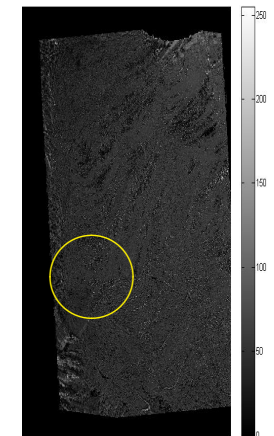

(B5) $\left|T_{\text {reg }}^{\mathrm{NGF}-\text { Curv }}-R\right|$

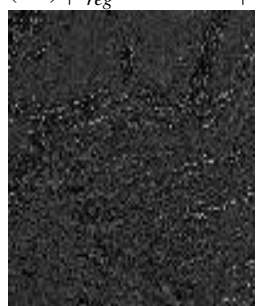

(C5) Highlight (B5)

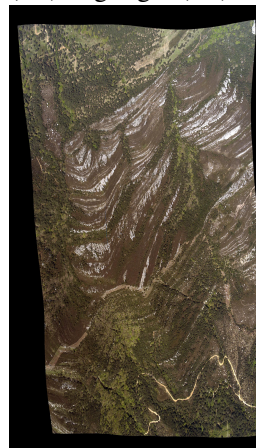

(D5) $T_{\text {reg }}^{\mathrm{NGF}-\mathrm{Curv}}$

Fig. 2.4 Image registration of an aerial photograph onto a hyperspectral image in the case of rugged terrain (scale $2400 \times 1800 \mathrm{~m}^{2}$ ). The first row shows (A1) a hyperspectral reference image $R$; (A2) an aerial photograph template image $T$; the second row shows (B0) a map of difference between these images (i.e. $|T-R|$ ) for methods NCC, MI, NGF, SURF and NGF-Curv. The third row (C0) zooms in on the circular regions of (B0). The last row of panels shows the aerial photograph template image after it has been registered using methods (D1) NCC, (D2) MI, (D3) NGF, (D4) SURF and (D5) NGF-Curv. 

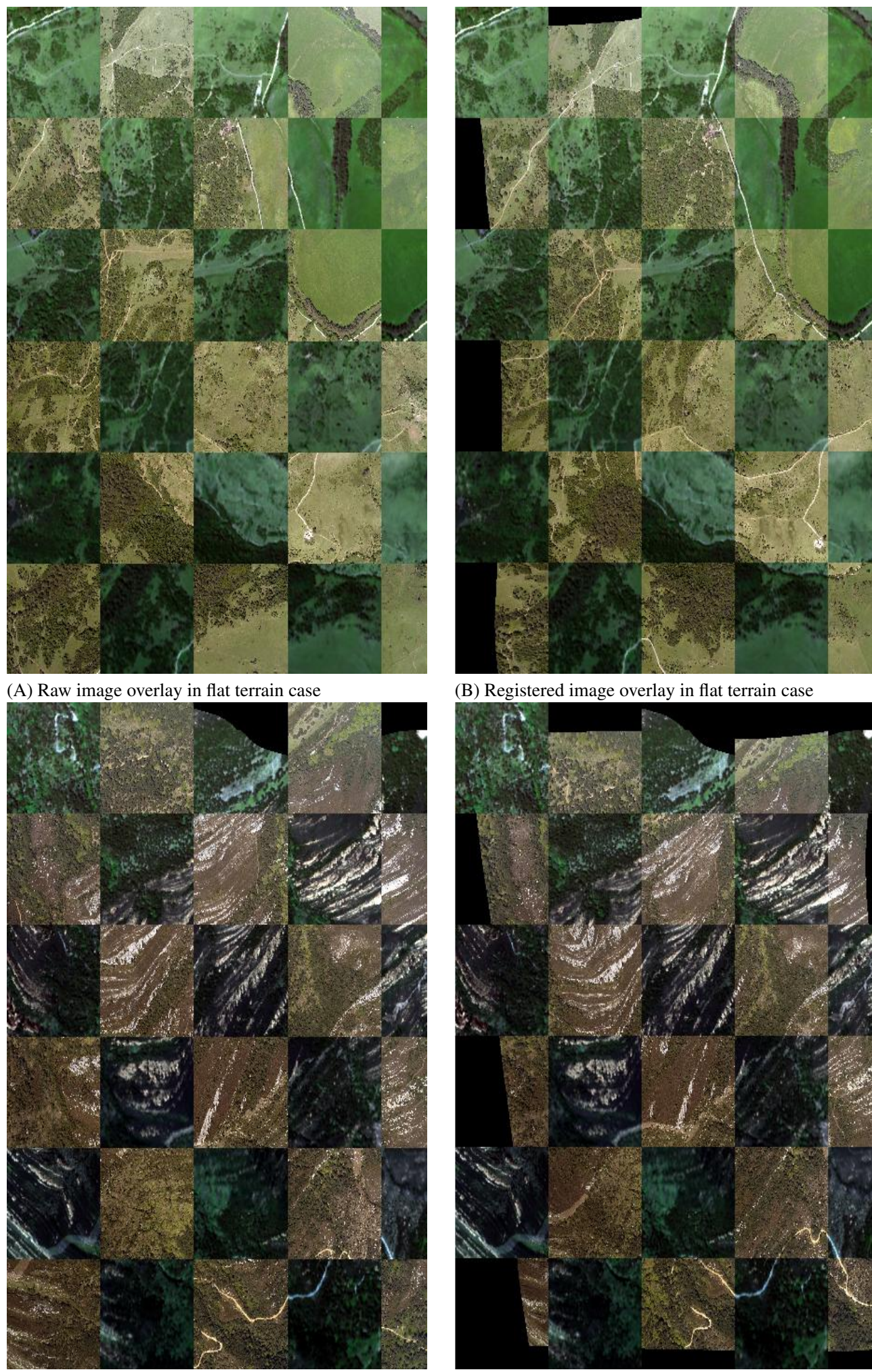

(B) Registered image overlay in flat terrain case

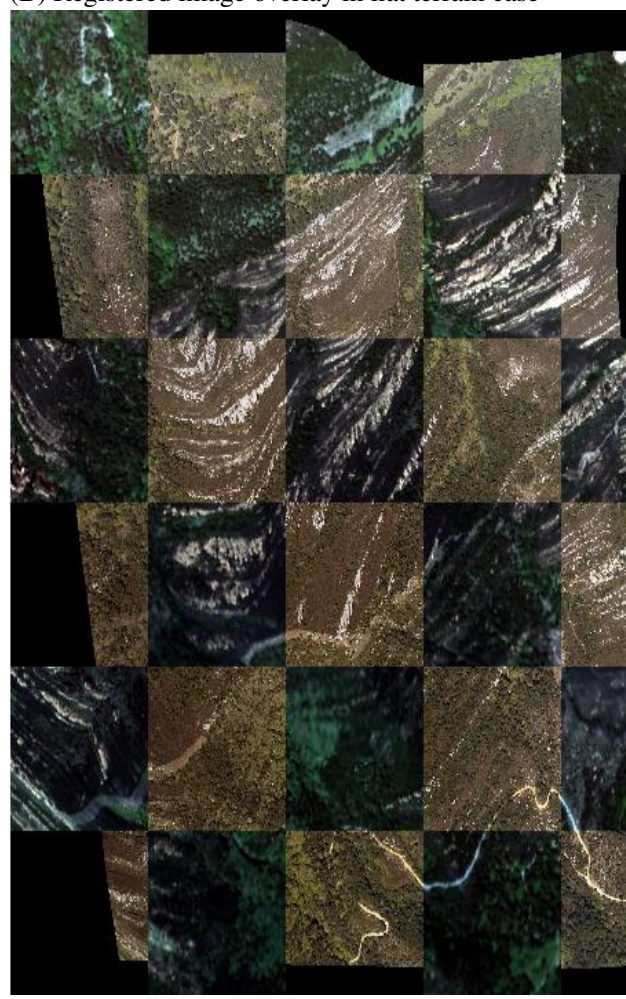

(C) Raw image overlay in rugged terrain case

(D) Registered image overlay in rugged terrain case

Fig. 2.5 Checkerboard overlay between aerial photograph template images $T$ and hyperspectral reference images $R$ and checkerboard overlay of registered aerial photograph template images $\left(T_{r e g}^{\mathrm{NGF}-\mathrm{Curv}}\right)$ and hyperspectral reference images $(R)$ generated by our NGF-Curv approach. (A)-(B) Flat terrain case; (C)-(D) rugged terrain case. 
Non-parametric image registration of airborne LiDAR, hyperspectral and photographic 50 present two image registration examples: one for a flat terrain and one for a rugged

We present two image registration examples: one for a flat terrain and one for a rugged terrain (Figures 2.3 and 2.4, respectively). Where topographical variation is large the correct alignment of the images becomes more difficult [47, 180, 265, 50]. The NGF-Curv registration approach (NGF-Curv) worked well in the case of flat terrain (see Figure 2.3), while parametric registration with three different distance measures (NCC, MI and NGF) and feature-based SURF poorly matched the detailed structures of a given reference image, see Figure 2.3 (B1)-(B5) in particular the parts marked by circles which are highlighted and given in Figure 2.3 (C1)-(C5). NGF-Curv method provides reasonable outcomes while parametric registration methods (NCC, MI and NGF) and SURF make serious mistakes and in particular, could not align detailed features (e.g. see Figure 2.4 (C1)-(C5)). In addition, the quantitative analysis in Table 2.1 supports NGF-Curv outperforms other methods in both flat and rugged cases (Figure 2.3 and Figure 2.4) although computational time of NGF-Curv is much slower than other methods. Figure 2.5 shows the results of aligning the aerial photographs onto the hyperspectral image for the cases of flat and rugged terrains in the form of a checkerboard: if the alignment is good then features such as roads and rivers should join across the checkerboard. We can clearly see that NGF-Curv method gives very accurate registration results.

As aerial photographs were registered individually onto hyperspectral imagery there may be mismatches at the edge of each aerial photograph (visible in Figures 2.3 and 2.4), which may produce noticeable discontinuity between the photographs. For example, in Figure 2.6 (C), the part marked by the red circle shows discontinuity at the interface of two aerial photographs. These boundary artifacts are due to a non-optimal choice of the regularisation parameter for the registration of aerial photographs to hyperspectral images. We chose to have a fixed regularisation parameter $\alpha$ in (NGF-Curv) which might not be optimal for every aerial photo in the dataset, and this caused errors at the boundaries. Tuning the parameters for each aerial photograph where discontinuity deteriorates the quality of registration, can 
improve the result significantly. In the case of the mismatch inside the circle in Figure 2.6 (C) (see (E3) for the highlight of its circle region) a tuning of the regularisation parameter $\alpha$ from $1.5 \times 10^{5}$ to $2 \times 10^{5}$ significantly improved the registration and removed the discontinuity between the two aerial photos (Figure 2.6 (D) and (E4)). The registration parameter $\alpha$ is sensitive to the scale of $0.1 \times 10^{5}$, trial and error method could find the parameter reasonably fast.

\subsection{Concluding remarks}

The experiments illustrated in Figures 2.3, 2.4 and 2.5 indicate that NGF-Curv image registration techniques can effectively co-align remote sensing images, working as well as established methods when registration is straight forward and out-performing those approaches when dealing with non-georeferenced photos. Remote sensing images are usually preprocessed before being sent to users, but the orthorectification and georeferencing procedures are not accurate enough to identify individual trees. Therefore, high accuracy registration methods for remote sensing datasets of wooded landscapes are needed.

Techniques based on feature extractions are well established in the field and are capable of accurate data assimilation in many applications. However, these approaches are difficult to apply to multi-sensor data, because different types of sensors may show different features, and sensor distortions are complex. Intensity-based parametric methods (such as NCC, MI and NGF) can perform fully automatic registration but assume that data are pre-processed and displacement between template and reference images is small. Feature-based parametric methods, for example SURF, are automatic if the main features are selected both in reference and template images. But since they depend on the features and the parametric transformation, their performance is reduced if there are many local distortions in the data.

NGF-Curv image registration provides a flexible approach allowing image registration with little prior knowledge of degree of distortion within the reference and template images. 
Non-parametric image registration of airborne LiDAR, hyperspectral and photographic imagery of wooded landscapes
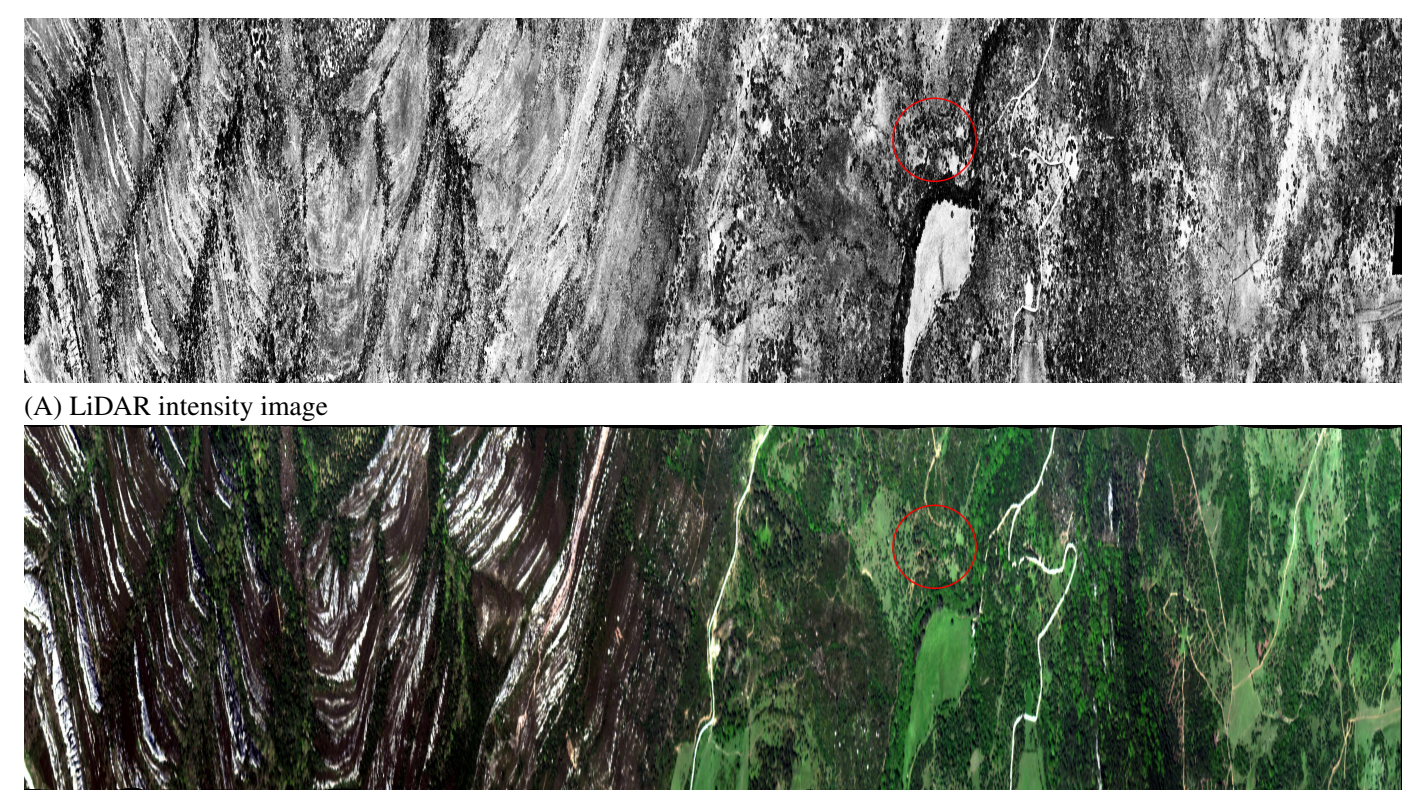

(B) RGB bands of registered hyperspectral image

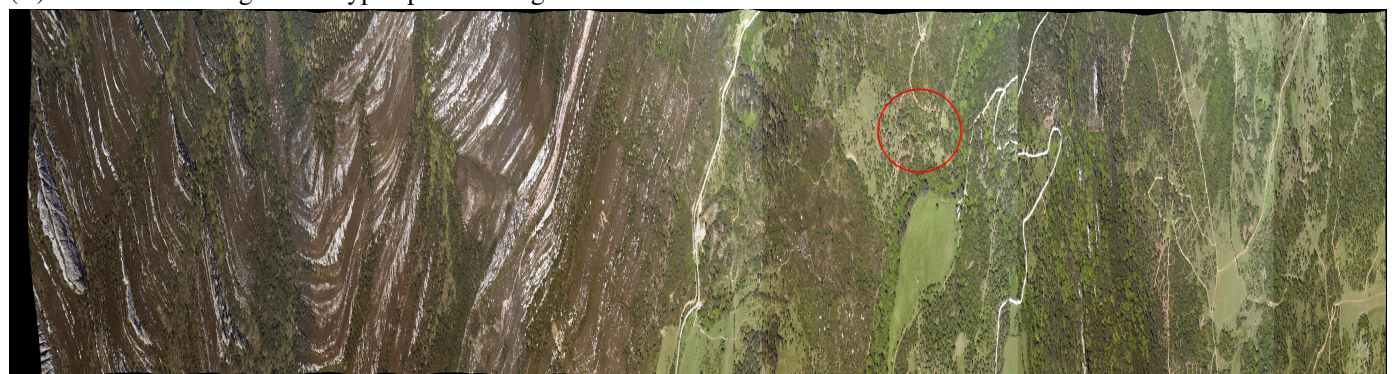

(C) Mosaic image of registered aerial photographs (with fixed global parameter $\alpha$ in NGF-Curv)

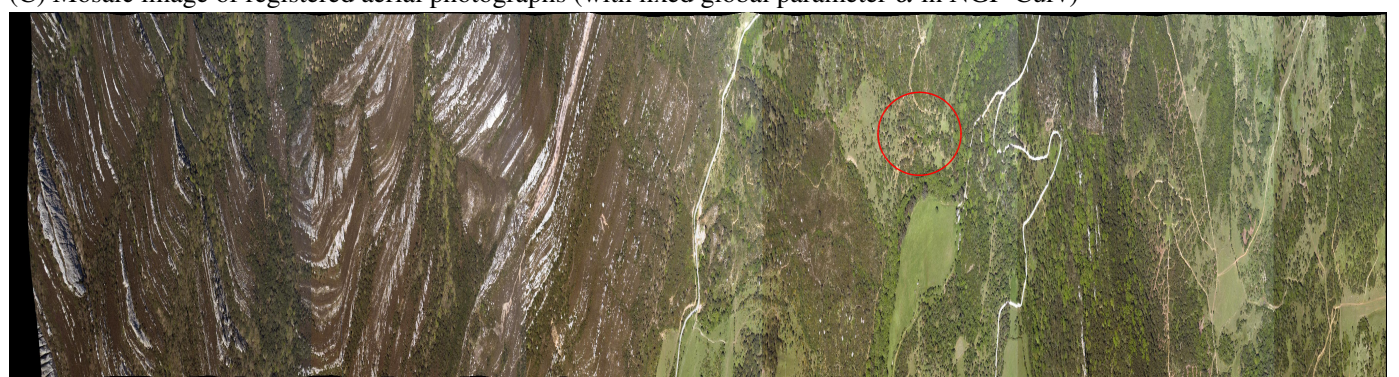

(D) Mosaic image of registered aerial photographs (with locally tuned parameter $\alpha$ in NGF-Curv)

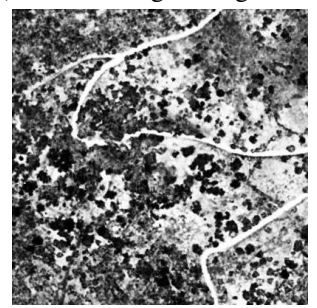

(E1) Highlight (A)

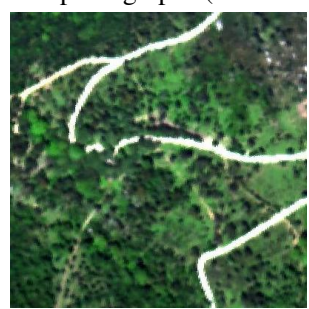

(E2) Highlight (B)

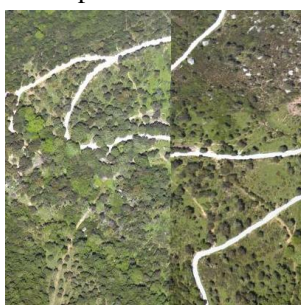

(E3) Highlight (C)

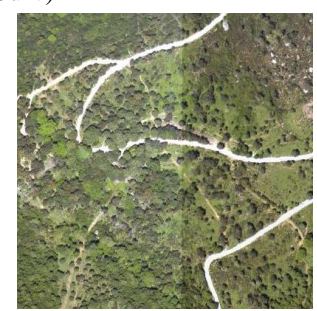

(E4) Highlight (D)

Fig. 2.6 Fully registered LiDAR, hyperspectral and aerial photograph imagery. (A) LiDAR intensity image; (B) RGB bands of hyperspectral imagery; (C) mosaic imagery of registered aerial photographs of our NGF-Curv approach with fixed global regularisation parameter $\alpha$; (D) mosaic imagery of registered aerial photographs of the NGF-Curv approach with locally tuned regularisation parameter $\alpha$; (E1)-(E4) highlights of the circle regions in (A)-(D). 
Validation of NGF-Curv with a variational formulation was demonstrated by numerical experiment in Section 2.5. This approach can be applied to non-orthorectified images with rough geographic boundary approximation. Although most images can be registered with a fixed regularisation parameter, it may lead to mis-alignment at the edge of the image (see Figure 2.6). Further research is required to find the regularisation parameter automatically, thus minimising mis-alignment at the boundary.

The NGF-Curv method is designed to measure similarity between images taken by multisensors, and because it compares gradients of two images, it can handle different types of imagery. Other similarity measures, such as Sum of Squared Distance and NCC perform poorly when applied to different types of images. The MI method (derived from information theory) is widely used as a similarity measure in remote sensing applications as it can be applied to multi-sensor imaging. It measures the joint probability of image intensities and can be viewed as a generalised similarity measure [194]. However, the MI method is highly non-convex, so it is difficult to optimise and increases the non-linearity of registration [118]. Since MI is based on joint density of intensity values, it may also suffer from interpolation induced artifacts [59].

Regularisation is key to the success of the NGF-Curv. Although a number of studies have used intensity-based similarity measures [271, 58, 59, 247, 209], the ill-posedness of these measures prevents their use in flexible applications in remote sensing. This means that successful image registration is conditional upon the data being preprocessed and displacement between images being small. In theory, adding a regularisation term makes the problem close to, or exactly, well-posed such that the registration problem has a meaningful solution; in reality though, it is difficult to remove all local minima using this approach, so exact solutions are rarely achieved in reality. A few regularisation methods have been suggested to guarantee well-posedness during the registration process [51]. As we mentioned before, most of these regularisation techniques are sensitive to affine linear displacement such 
Non-parametric image registration of airborne LiDAR, hyperspectral and photographic imagery of wooded landscapes

that pre-registration with affine linear transformation is required $[193,99,98]$. In contrast, curvature regularisation used here does not require affine preregistration steps. However, affine parametric registration at coarsest level is recommended in general applications, as NGF-Curv still penalises affine transformation by its boundary conditions (i.e. it is still influenced by initial position of two images to some extent, see[194, 123]).

Some aspects of NGF-Curv require further refinement. As is often the case with image registration, the NGF-Curv method is dependent on the quality of the reference image. We used LiDAR intensity images in order to register hyperspectral image. We believe the quality of LiDAR can be further improved by increasing the understanding of the radiometric properties of LiDAR intensity: LiDAR pulses do not only backscatter off the upper canopy leaves but also internal structures through penetration, and if we could filter for the information from the upper canopy, then the intensities of the LiDAR would be more similar to those from the camera or hyperspectral sensor. However, currently the automatic gain control (AGC) of Leica systems adjusts the pulse energy during the LiDAR acquisition (i.e. the pulse energy is increased when the returned energy is low). An AGC value within the range $[0,255]$ is given for each pulse in the LAS file, and a few studies have attempted to normalise LiDAR intensity using these numbers $[149,156,155]$. Whilst none of those methods are able to successfully correct the LiDAR datasets we used, we believe that a successful radiometric calibration could indeed improve the registration accuracy.

Another difficulty is that hyperspectral and aerial photos are strongly influenced by shading effects, because they record backscattered solar energy. If imagery is collected early or late in the day, then shaded pixels create strong gradients on one side of trees, so the registration process is intrinsically biased to some extent. Combining image registration with shade removal [97] could improve the quality of image registration.

Although further research on finding the regularisation parameter automatically is required, this research has introduced a fresh insight on image registration of remote sensing. 


\section{Chapter 3}

\section{D individual tree segmentation of}

\section{integrated airborne LiDAR and optical imagery using normalised cut with priors}

\subsection{Introduction}

Remote sensing is a well-established approach in large-scale geophysical and environmental research [88]. Until recently, vegetation characteristics obtained by remote sensing data have been rasterised in 2D maps, with each pixel summarizing information from many individual plants within them $[67,17]$. For example, NASA's Landsat 8 satellite produces imagery at 30m spatial resolution, which is too coarse to detect individual trees. However, earth observation technology is producing information at increasingly high spatial resolution. In particular, airborne LiDAR produces a 3D point cloud indicating where laser pulses emitted from the transceiver have reflected off leaves, branches and the forest ground, making it possible to map individual trees over tens of thousands of hectares $[60,122,71]$. In addition, airborne hyperspectral imagery can be used to estimate the physical and chemical properties of canopies, and when used alongside with LiDAR can map these properties down to the 
3D individual tree segmentation of integrated airborne LiDAR and optical imagery using normalised cut with priors

level of individual tree crowns [15]. This "spectranomic" approach has been used to map invasive tree species in Hawaiian rainforests $[12,16]$ and to quantify the spatial variation in biochemical diversity in tropical regions $[17,19]$. Although tree-level detail is not necessary for many applications, airborne remote sensing approaches are already integrated into some national forest inventory programs (e.g. Finland [251]), and tree-centric approaches have the potential to revolutionise forest research, by keeping track of individual responses to pest and pathogen outbreaks, invasive species and climate change.

A limitation to a wider use of tree-centric approaches is that delineation of individual tree crowns (ITCs) remains inaccurate for different forest types. For example, ITC delineation in a temperate foreset is challenging because canopy height is uniform in the forests [122, 140,71]. Classical delineation approaches work with rasterized canopy height models (CHMs) derived from the LiDAR point cloud. These methods include watershed algorithms $[60,152,159$, 266], variable window filtering [139, 240], multi-scale edge segmentation [39], and attentive vision methods [207]. All these approaches share several problems: (a) the smoothing process determines the number of trees detected in the CHM: too strong a smoothing factor leads to under-segmentation, while one which is too small can generate many tree-like artifacts[189]; (b) sub-canopy trees are difficult to detect as they all rely solely on canopy surface geometry unless LiDAR vertical profiles are considered [86]; and (c) the interpolation and smoothing processes involved in generating CHMs result in underestimation of tree heights, meaning that additional post-processing is needed to rectify the results $[240,152]$. In order to face such problems more advanced methods that exploit the entire LiDAR point cloud have been developed. These methods include $k$-mean clustering [197], normalised cut (NC) [237, 256, 224, 264], adaptive clustering [167], support vector machine (SVM) $[236,270]$, and relative spacing between trees [179]. These methods appear to perform well in coniferous trees in which conical crowns are easily distinguished, although comparisons have not yet been made using independent benchmark datasets [204]. Their performance 
in tropical or temperate forests has not yet been evaluated, but broadleaf species in these forests have non-conical crown which intermingle, presenting a much greater challenges to segmentation algorithms [224, 264, 179, 204].

In principle, the fusion of high resolution optical imagery with LiDAR data should lead to improvements in ITC delineation $[60,152,159,139,240,39,75,266]$ by distinguishing neighbouring trees through differences in their radiometric properties $[149,156,155]$. Airborne multi- and hyperspectral imagery or aerial photographs could all be used for this purpose, as long as their spatial resolution is high enough (i.e. the pixel size is smaller than the minimum crown size that we need to detect) $[151,245,130,40,71,122,83,143]$. However, multi-sensor approaches are only possible if the different data are accurately co-aligned, thus image registration must be applied prior to their fusion (see [80, 164]). A second issue is that extracting feature information directly from high dimensional data - such as the multiand hyperspectral datasets - often leads to inaccurate results [74]. Therefore, dimensional reduction is required before applying any algorithm, or using feature extraction techniques like principal component analysis (PCA) [53], or through feature selection over the original bands $[74,75]$.

This study sought to overcome some of the issues surrounding ITC delineation, by developing a new approach called MCNCP-RNC (i.e. MultiClass Normalised Cut with Priors followed by Recursive Normalised Cut), based on NC [237, 256, 224, 264]. NC is a well-established approach in image analysis for clustering points, which finds an optimal way of separating points (or pixels) into disjoint clusters based on a similarity between the points. It starts with a matrix of similarity measure between all possible pairs of points, and uses the eigenvectors of that matrix to group points into clusters [237]. In the NC scheme, the clustering is seen as a graph partitioning problem. The nodes of the graph are the points and the weight on a graph edge connecting two nodes is their similarity. In the case of LiDAR data, the similarity matrix is derived from the physical distance between points in 3D space. 
3D individual tree segmentation of integrated airborne LiDAR and optical imagery using normalised cut with priors

In the case of hyperspectral data, the matrix is derived from their radiometric similarity and distances between pixels in 2D space. NC seeks to partition the graph into subgraphs with high similarities between the nodes of the same subgraphs and a low similarities between nodes from different subgraphs. The advantage of the NC approach is that graph weights can be defined using optical imagery alongside LiDAR, thus providing a framework for fusing different types of remote sensing datasets.

The study's main objectives are (a) to describe MCNCP-RNC, an efficient way of fusing LiDAR data and optical imagery, and examine whether optical imagery can guide ITC delineation along with LiDAR data; (b) to delineate ITCs directly from the 3D LiDAR point cloud thus avoiding interpolation related problems; (c) to examine the capability of detecting understory trees from the LiDAR point cloud; and (d) to validate the accuracy of ITC delineation in coniferous and broadleaved woodlands. The paper is organized as follows: in Section 3.2, the general NC framework is explained. Our ITC delineation method MCNCPRNC is then introduced in Section 3.3. The test datasets used to exemplify our approach are described in Section 3.4. The performance of our approach is evaluated in Section 3.5. Section 3.6 discusses our approach in relation to others and gives recommendations for future work.

\subsection{The theoretical background of normalised cut frame- work}

This section summarises the NC framework formally [237, 224], and explains how it is adapted for multi-sensor imagery. A graph $G$ is a pair of sets, $G=(V, \varepsilon)$, where $V$ is the set of vertices and $\varepsilon$ is the set of edges. Each edge $w_{i j} \in \varepsilon$ corresponds to a non-negative similarity weight between two vertices $i, j \in V$. The objective of graph cut is to partition the graph into two disjoint sets $A$ and $B$ by cutting edges that connect the two sets, such 
that $A \cup B=V$ and $A \cap B=\emptyset$. These two disjoint sets could then be separated into four, by applying the cut to each of them, and further subdivisions could occur until some pre-defined end-point is reached. By this recursive application of graph cut, the individual trees in the forest can be delineated. We define the cut as:

$$
\operatorname{cut}(A, B)=\sum_{i \in A, j \in B} w_{i, j}
$$

that is, the sum over the weights of all edges that connect $A$ and $B$. Moreover, we define $\operatorname{assoc}(C, V)=\sum_{i \in C, j \in V} w_{i j}$ to be the total weight of connections from nodes in $C$ to all nodes in the graph. The NC method finds sets $A$ and $B$ by minimising the following energy:

$$
\operatorname{Ncut}(A, B)=\frac{\operatorname{cut}(A, B)}{\operatorname{assoc}(A, V)}+\frac{\operatorname{cut}(A, B)}{\operatorname{assoc}(B, V)} .
$$

Let $|V|=N, D \in \mathbb{R}^{N \times N}$ be a diagonal matrix with diagonal entries $d_{i}=\sum_{j=1}^{N} w_{i j}, W \in$ $\mathbb{R}^{N \times N}$ be a symmetric matrix with entities $w_{i j}$, let $x \in \mathbb{R}^{N}$, and $1 \in \mathbb{R}^{N}$ be all-ones vector. In order to solve (3.2), we can reformulate it as the following problem:

$$
\begin{aligned}
& \min _{x \in \mathbb{R}^{N}} x^{T} D^{-\frac{1}{2}}(D-W) D^{-\frac{1}{2}} x \\
& \text { s.t. } \quad x^{T} D^{\frac{1}{2}} 1=0, \quad x^{T} x=1,
\end{aligned}
$$

obtaining the segmentation by splitting $V$ into two sets by thresholding the solution $x$ (for example by taking the mean or median of $x$ ). The eigenvector corresponding to the second smallest eigenvalue of the matrix $D^{-\frac{1}{2}}(D-W) D^{-\frac{1}{2}}$ gives the solution of (3.3). The equation (3.3) can be used for multiclass setting by splitting $V$ into multiple sets recursively, i.e. repeatedly apply the binary cut to every smaller subset until some predefined end-point is reached. Specifically, the solution of (3.3) gives two disjoint subsets, and each of those subsets can be further split, with the decision as to whether or not to make the split depending on whether the $N c u t$ value exceeds some predetermined threshold [237]. Reitberger et al. 
3D individual tree segmentation of integrated airborne LiDAR and optical imagery using normalised cut with priors

used recursive NC (equation (3.3)) to delineate trees from a LiDAR point cloud [224]. The recursive scheme is computationally inefficient because it needs to solve equation (3.3) repeatedly until it reaches a predefined threshold. Since LiDAR data contains millions of points per hectare, recursive graph cut requires huge computational power to work on datasets larger than a few square metres. In addition, an issue with the recursive approach is that equation (3.3) uses only the second smallest eigenvector [237, 256], discarding information from subsequent eigenvectors that could help refine the partitioning. Finally, it is difficult to incorporate priors using this approach.

Alternatively, the binary NC problem (3.3) can be extended to a multiclass problem, although this requires to define in advance the number of classes [256]. Let $X=\left(x_{1}, \cdots, x_{C}\right) \in$ $\mathbb{R}^{N \times C}$ where $C$ be the number of clusters. Then, the multiclass NC problem can be expressed in a similar way to problem (3.3):

$$
\begin{aligned}
\min _{X \in \mathbb{R}^{N \times C}} \operatorname{tr}\left(X^{T} D^{-\frac{1}{2}}(D-W) D^{-\frac{1}{2}} X\right) \\
\text { s.t. } \quad x_{i}^{T} D^{\frac{1}{2}} 1=0, x_{i}^{T} x_{i}=1, \quad i=1, \ldots, C,
\end{aligned}
$$

splitting $V$ into $C$ sets by the clustering solution $X$ using either $k$-means or spectral rotation. This multiclass approach is computationally efficient, since the number of clusters is fixed at $C$, equation (3.4) needs to be solved only once. However, this approach is also problematic as the number of trees should be known in advance. Moreover, it remains unclear how to include priors into the decision process in equation (3.4).

To solve these problems, we first estimate the locations of tree tops from the local maxima of the CHM and use these locations as priors, providing method (3.4) with an estimate of the number of clusters and their positions. Constrained NC has been proposed by [133] but has never been used for ITCs delineation. This scheme regards a prior as an additional constraint to the solution of (3.4), minimising NC energy but also satisfying the condition 
that the correlation between the solution and the prior is larger than or equal to a predefined value $(\kappa)$.

Let $S=\left(s_{1}, \cdots, s_{C}\right) \in \mathbb{R}^{N \times C}$ be unary priors, then the MultiClass Normalised Cut with Priors (MCNCP) approach is minimising

$$
\begin{aligned}
\min _{X \in \mathbb{R}^{N \times C}} \operatorname{tr}\left(X^{T} D^{-\frac{1}{2}}(D-W) D^{-\frac{1}{2}} X\right) \\
\quad \text { s.t. } \quad x_{i}^{T} D^{\frac{1}{2}} 1=0, x_{i}^{T} x_{i}=1, x_{i}^{T} s_{i} \geq \kappa, \quad i=1, \ldots, C,
\end{aligned}
$$

where $\kappa$ is a correlation parameter, the splitting of $V$ into $C$ sets is determined by the clustering $X$. The solution of equation (3.5) gives $C$ separate clusters of data, i.e. ITCs in our case. The correlation term is a hard constraint, which must be satisfied. In other words, the solution must have $C$ non-empty disjoint clusters. However clusters could be merged in some cases, probably because priors are located too closely together in space. This method is much faster and efficient than solving binary clustering recursively because we fixed the number of clusters and need to solve equation (3.5) just once. If the priors underestimate the actual number of trees present, then the clusters obtained by equation (3.5) will not represent realistic ITCs. In this case, we can can apply the recursive NC in equation (3.3), which will be explained in Section 3.3.

\subsection{Methods}

Our data processing pipeline is shown in Figure 3.1. First, LiDAR data and optical imagery were registered using the NGF-Curv method in Chapter 2. Then, a state-of-the-art feature reduction method, such as robust PCA (rPCA) [53], is used to reduce the number of features of the co-aligned dataset. Next, our new segmentation method - Multi Class Normalised Cut with Priors followed by Recursive Normalised Cut (MCNCP-RNC) is introduced. We emphasize again that our method delineates ITCs directly from the 3D LiDAR point cloud alongside 
3D individual tree segmentation of integrated airborne LiDAR and optical imagery using normalised cut with priors

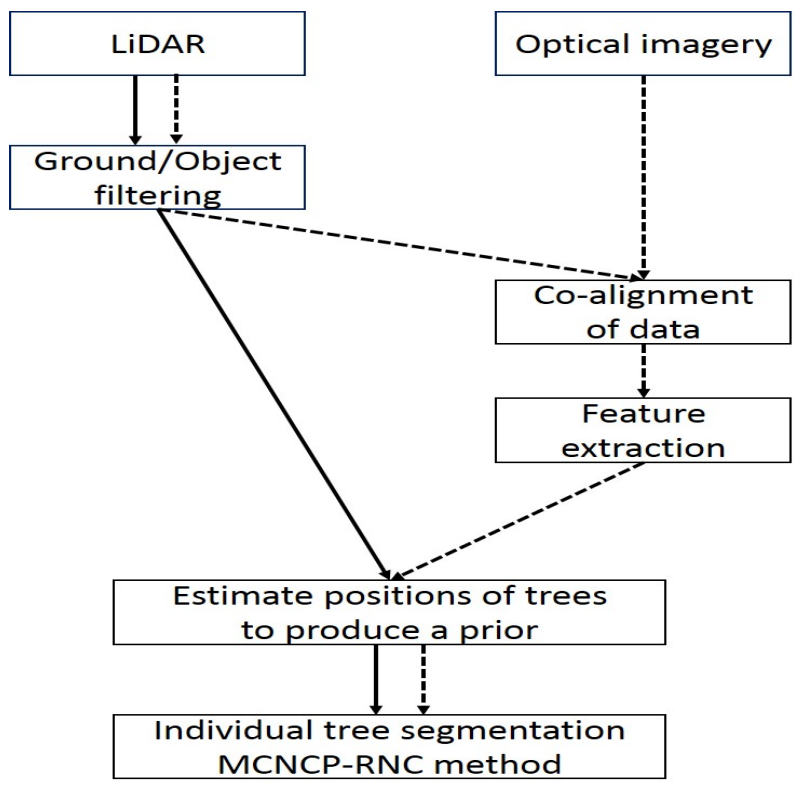

Fig. 3.1 The workflow of individual tree segmentation using LiDAR only (solid line), and LiDAR with hyperspectral imagery (dashed line).

with optical imagery, therefore ITCs are not influenced by interpolation or smoothing errors prevalent in CHM approaches, and predicting forest parameters (e.g. individual crown volumes) more accurately.

Our proposed ITCs delineation method consists of multiple steps, each step is dedicated to a particular process in the overall workflow. In the following section we explain each step in Figure 3.1 in detail.

\subsubsection{Registration of remote sensing datasets}

LiDAR data and hyperspectral imagery are not usually precisely co-aligned when delivered by the data provider. Camera direction, topography and lens distortion all affect the quality of hyperspectral imagery, and LiDAR boresight is usually more accurate than that of the hyperspectral sensor, so inaccuracies remain even after geometric correction. To co-align 
these data, registration of LiDAR and optical imagery can be conducted using NGF-Curv algorithm in Chapter 2.

\subsubsection{Feature extraction}

Combining different data sources can produce high dimensionality datasets, increasing the computation effort and making it more difficult to exploit their information content [74]. To alleviate this problem, the rPCA feature reduction technique is used in order to reduce the high dimensionality features space to a few meaningful features. Conventional PCA is sensitive to the magnitude of noise in data. In contrast, rPCA is designed to robustly recover a low rank matrix $L$ from a corrupted measurement matrix $M$ [53].

In our case, $M$ is composed of hyperspectral images. rPCA is represented as the following minimization problem:

$$
\min _{L, S}\left\{\operatorname{rank}(L)+\lambda\|S\|_{0}\right\} \quad \text { s.t. } M=L+S
$$

where $\|\cdot\|_{0}$ is the $l_{0}$-norm which imposes sparsity property on $S, \operatorname{rank}(\cdot)$ is the dimensions of vector spaces spanned by columns or rows of a matrix, and $\lambda$ is a regularisation parameter. Since this optimisation problem is intractable, in general, the rank and the $l_{0}$-norm are usually replaced by the nuclear norm $\|\cdot\|_{*}$ (sum of singular values) and the $l_{1}$-norm (sum of the absolute values of the whole entries) respectively. This results in the following convex problem,

$$
\min _{L, S}\left\{\|L\|_{*}+\lambda\|S\|_{1}\right\} \quad \text { s.t. } M=L+S
$$

For a solution $(L, S)$ of (3.6), $L$ contains meaningful features, and $S$ contains sparse outliers. As the objective function is convex, it can be solved by various convex optimisation algorithms. In this paper, the alternating direction method of multipliers was used [267]. As we were interested in meaningful features rather than sparse outliers, we extracted the low 
3D individual tree segmentation of integrated airborne LiDAR and optical imagery using normalised cut with priors

rank parts $L$, which corresponds to principal components in classic PCA. The first principal component was ignored because it contained illumination information instead of key features of ITCs [250]. In this paper, two to five principal components were extracted and assigned to corresponding LiDAR tree object points by using horizontal geospatial coordinates. If there is more than one LiDAR point in a pixel of hyperspectral imagery, then all points in the pixel were assigned the same rPCA coefficient.

\subsubsection{Local maxima detection}

Local maxima within the LiDAR point cloud provide the prior information on tree locations in this paper. Those local maxima can be easily extracted from either the LiDAR point cloud directly or from the rasterized CHM, using a moving window approach [139] or a watershed approach [60]. In our analysis, we adopted a marker-based watershed approach for tree top detection implemented in the toolbox for LiDAR data filtering and a standard forest analysis (TIFFS, Globalidar ltd.) [60]. All LiDAR points within $0.7 \mathrm{~m}$ radius of each local maxima were clustered together. We used these clusters as priors, thus enforcing the solution of equation (3.5) correlated with these priors. The marker-based watershed approach is just one of the possible methods to set up priors and the users can choose any other method to set them up [224] (see Section 3.5).

\subsubsection{MultiClass Normalised Cut with Priors (MCNCP)}

To build the graph for NC, proper weights need to be assigned to the vertices given by the LiDAR points. For computing the weights of the graph, we use a normalised weight as a function of the Euclidean distance in horizontal $(x, y)$, vertical $(z)$ and hyperspectral features $(f t s)$ of the $(x, y)$ coordinates, with bandwidth parameters, $\sigma_{x y}, \sigma_{z}, \sigma_{f t s}$, balancing 
these components, i.e.

$$
w_{i j}=e^{\frac{\left\|(x y)_{i}-(x, y)_{j}\right\|}{\sigma_{x, y}^{2}}} \times e^{\frac{\left\|z_{i}-z_{j}\right\|}{\sigma_{z}^{2}}} \times e^{\frac{\left\|f t s_{i}-f t s_{j}\right\|}{\sigma_{f t s}^{2}}}
$$

For constructing the graph, we observe that a fully connected graph requires $\mathscr{O}\left(n^{2}\right)$ memory complexity, which is not practical. Instead, a $d$-neighbourhood sampling strategy is adopted, where similarity is computed only within a radius $d$. In our examples, $d$ ranged from $0.5 \mathrm{~m}$ to $2 \mathrm{~m}$ depending on the point density of LiDAR (lower radii at higher densities to reduce the memory costs). The equation (3.5) (MCNCP) was solved with a $d$-neighbourhood similarity matrix and pre-defined clusters taken from the local maxima.

The MCNCP approach, as described so far, segments the 3D LiDAR point cloud into the same number of tree crowns as identified by traditional CHM-based methods, because this information is used as a prior. Thus, MCNCP suffers from the same problems as classic approaches in terms of failing to detect understory trees. However, the RNC method (3.3) described in Section 3.2 is very effective at ITC delineation, including the detection of understory trees[224], but at a computational cost. For this reason, we included an optional RNC, to be applied after the initial MCNCP, which can further separate individual trees, as well as filtering outliers. Therefore, the RNC method with a low threshold energy as defined earlier in Section 3.2 is available for filtering outliers and detecting sub-canopy trees in a post-processing step after the MCNCP method has been applied. We suggest to use this two-step approach, which we called MCNCP-RNC (MultiClass Normalised Cut with Priors followed by Recursive Normalised Cut).

\subsection{Datasets description and design of experiments}

The accuracy of our algorithm was tested on (a) a set forest plots located in the Alps which form part of the NewFor benchmarking project, established specifically for the purpose of 
3D individual tree segmentation of integrated airborne LiDAR and optical imagery using normalised cut with priors

comparing ITC algorithms [204], (b) a coniferous forest located near Trento in the Italian Alps, and (c) a lowland deciduous forest located near Oxford, UK.

- The NewFor LiDAR Single Tree Detection Benchmark Dataset consists of LiDAR and ground-truth information from 14 survey sites in the Alps (10 pilot areas in 6 countries) [89]. A major advantage of working with the NewFor benchmark dataset is that it provides an objective means of comparing our approach with others, and includes sophisticated validation software with which to evaluate algorithms by matching ITCs derived from LiDAR with known tree locations in the field. The ground truth data were provided with geocoordinates, tree height, $\mathrm{DBH}$ and canopy volume information. The errors of geocoordinates were less than 1 metre. The LiDAR point density was more than 10 per $\mathrm{m}^{2}$ in 12 out of 14 study site. The ranges of the LiDAR point density in the benchmark dataset were from 4 to 121 per $\mathrm{m}^{2}$. A disadvantage of the NewFor dataset with regard to our proposed delineation procedure is that it does not provide any optical imagery (i.e. we worked with pipeline shown with solid lines in Figure 3.1). Note that these datasets are primarily coniferous, which are relatively straightforward to delineate because conifers have distinct peaks to their crowns.

- The Italian dataset was collected from a location near Trento in the Alps. It consists of hyperspectral imagery, LiDAR data and ground-based tree maps for 7 plots dominated by coniferous trees. Each plot is a circle of $15 \mathrm{~m}$ radius. In these plots, all trees with DBH above $1 \mathrm{~cm}$ were accurately georeferenced by differential GPS and manually corrected with local reference trees from LiDAR data. The estimated error of the ground truth of tree positions was one metre. The hyperspectral imagery were collected with an AISA Eagle sensor, covering 400-970nm with 61 spectral bands, while the LiDAR data were acquired by a Riegl LMS-Q680i sensor at an unusually high point density $\left(\geq 87\right.$ points per $\mathrm{m}^{2}$ ) because LiDAR data were collected repeatedly from the 
study site. Hyperspectral data were collected the $13^{\text {th }}$ of June 2013, while LiDAR data were collected between $7^{\text {th }}$ and $9^{\text {th }}$ of September 2012.

- The English dataset was collected from Wytham wood, Oxfordshire, England $\left(51^{\circ} 46^{\prime} \mathrm{N}\right.$, $\left.1^{\circ} 20^{\prime} \mathrm{W}\right)$. A 18 -ha forest plot was established in this wood in 2008 using standardised methods used in an international network of Smithsonian Institution Global Earth Observatories (SIGEO) [52]. Each hectare was delimited into 25 subplots of $20 m \times$ $20 m$. Every tree larger than $5 \mathrm{~cm}$ diameter at chest height $(\mathrm{DBH})$ was tagged, its DBH measured, its species identified and its location mapped. There were 23 species of tree and shrub within the plot. In total, 20,308 stems and 16,313 individual trees were recorded (some trees had multiple stems). These plots were re-censused in 2009 and 2012, and the latest dataset was used in this study.

As it was only measured on 389 individuals of these trees, we used allometric equation to estimate tree height for all the other trees. Species-specific functions were fitted to the height -diameters relationships $(H=a \ln D B H+b$, where $a$ and $b$ are coefficients estimated by linear regression) and these functions were used to estimate tree height information from DBH. The estimated positioning error of the plot corners is approximately $2 \mathrm{~m}$, while tree positions are located within about $10 \mathrm{~m}$.

Hyperspectral imagery was collected in 24, June, 2014, simultaneously with LiDAR data by the airborne research and survey facility of the national environmental research council of UK (NERC-ARSF). The airplane flew at a nominal height above ground of approximate $800 \mathrm{~m}$. Hyperspectral imagery was obtained by AISA Fenix sensor, which is a pushbroom imaging array with 384 cross-track pixels and provides 361 spectral bands from the visible to shortwave infrared $(0.4-2.5 \mu \mathrm{m})$ region. The hyperspectral imagery was orthorectified and georeferenced in Ordinance Survey Great Britain (OSGB) projection, with spatial resolution of $1.2 \mathrm{~m}$ approximately. The airborne LiDAR data were acquired by Leica ALS-50 II sensor. The field of view was $12^{\circ}$. 
3D individual tree segmentation of integrated airborne LiDAR and optical imagery using normalised cut with priors

The LiDAR data were originally captured in full wave-form, however, they were converted to discrete LiDAR point cloud during the pre-processing step, within which the LiDAR point cloud was georeferenced in OSGB projection. The final point density was approximately 6 points $/ \mathrm{m}^{2}$. The equipment on board the NERC aircraft is regularly calibrated to ensure that LiDAR data were accurately georeferenced and the hyperspectral imagery is radiometrically calibrated before delivery.

The validation of the ITC delineation was conducted using the tree matching software provided by the NewFor project $[89,148]$, which compares relative positions and heights of segmented trees with those recorded in the ground plots. Specifically, it measures 2D Euclidean distance and height difference between ground truth and segmented trees. Groundtruth trees within $5 \mathrm{~m}$ of segmented trees, both horizontally and vertically, were considered as potential matches. The closest tree in both horizontal and vertical distances was selected as the match. By comparing not only tree positions but also heights, this validation software circumvents the inaccurate georeferencing of the ground truth. The sensitivity of the MCNCPRNC algorithm with respect to prior information was examined by comparing its results when the TIFFS watershed algorithm [60] and moving window filtering (MWF) [139] were used to establish the priors. In order to evaluate the performance of MCNCP-RNC, we compared our segmentation approach with RNC [224] and the CHM-based watershed algorithm of TIFFS [60]. The performance of TIFFS was equal or better than the best performance of others [89]. Thus we decided to compare our graph cut results with TIFFS only. Using TIFFS to provide priors on tree locations for MCNCP-RNC gave slightly more accurate results than using MWF in five out of seven Italian test plots (see Table 3.2). For this reason, all the remaining results are based on TIFFS.

MCNCP-RNC was evaluated using the LiDAR imagery in the NewFor benchmark, Italian and England datasets. We adopted trial-and-error to find optimal parameters of MCNCPRNC. Table 3.1 shows a set of parameters used for each dataset. When comparing RNC and 
Table 3.1 The parameter settings for the experiments

\begin{tabular}{l||c|c|c|c|c|c|}
\hline \multicolumn{1}{c||}{\multirow{2}{*}{\multicolumn{1}{c||}{ Dataset }}} & \multicolumn{3}{c|}{ MCNCP } & \multicolumn{3}{c|}{$\mathrm{RNC}$} \\
\cline { 2 - 8 } & $\sigma_{x y}$ & $\sigma_{z}$ & $\sigma_{f t s}$ & $\sigma_{x y}$ & $\sigma_{z}$ & $\sigma_{f t s}$ \\
\hline \hline Italian dataset & 1 & 3 & 0.005 & 0.5 & 2 & 0.005 \\
\hline NewFor benchmark & 2 & 5 & n/a & 2 & 5 & n/a \\
\hline English dataset & 2 & 3 & 0.005 & 2 & 3 & 0.005 \\
\hline
\end{tabular}

MCNCP-RNC with the Italian dataset, the parameters in MCNCP-RNC were set to $\sigma_{x y}=3$ and $\sigma_{z}=3$ for the MCNCP step, and $\sigma_{x y}=0.5$ and $\sigma_{z}=2$ for the RNC step, while for the hyperspectral imagery the parameter was set to $\sigma_{f t s}=0.005$ in both the MCNCP and RNC step. For the RNC method of the Italian dataset, parameters used were $\sigma_{x y}=1$ and $\sigma_{z}=3$. For the NewFor benchmark dataset, MCNCP-RNC parameters were set to $\sigma_{x y}=2, \sigma_{z}=5$ for both MCNCP and RNC steps. In the English dataset, the parameters used for MCNCP-RNC were set to $\sigma_{x y}=2, \sigma_{z}=3$ for LiDAR and $\sigma_{f t s}=0.005$ for the hyperspectral imagery.

\subsection{Results}

\subsubsection{Tree delineation using LiDAR imagery}

Considering the conventional CHM-based segmentation approaches, the watershed algorithm of TIFFS was found to provide the best performances for locating tree top priors on our datasets. When TIFFs and the MWF methods were used to segment the Italian dataset, TIFFS proved to be superior in plots 77, 102, 129, 220 and 292, and performed similarly well in the other two plots (see Tables 3.2 and 3.3). Comparing TIFFS with the eight delineation methods already explored using the NewFor benchmark datasets [204, 89].

Our method, which first applies a multi-class normalised cut with priors (MCNCP) and then applies RNC to each of the segmented point clouds (MCNCP-RNC), outperformed the RNC method without priors approach. Figures 3.2 and 3.3 illustrate the results of individual tree detection (in 2D and 3D respectively) based on MCNCP-RNC versus RNC. 
3D individual tree segmentation of integrated airborne LiDAR and optical imagery using normalised cut with priors

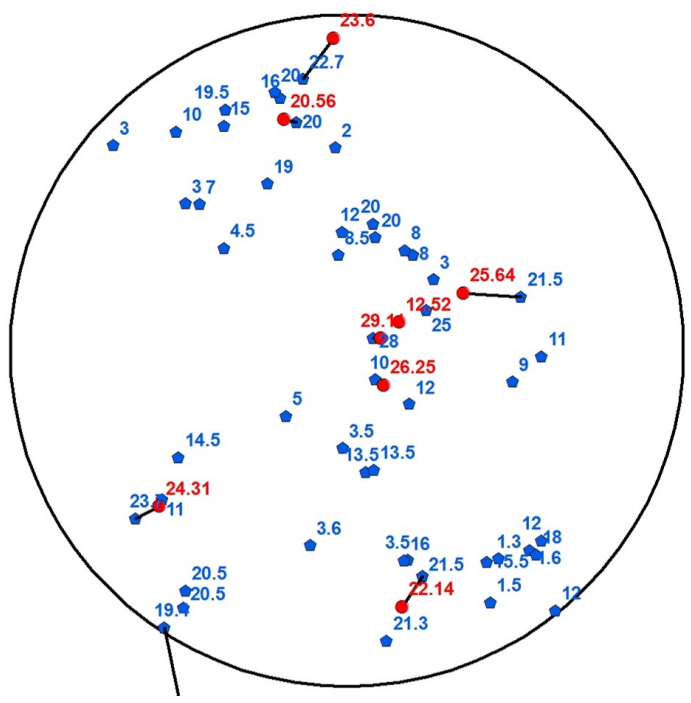

(a1) RNC

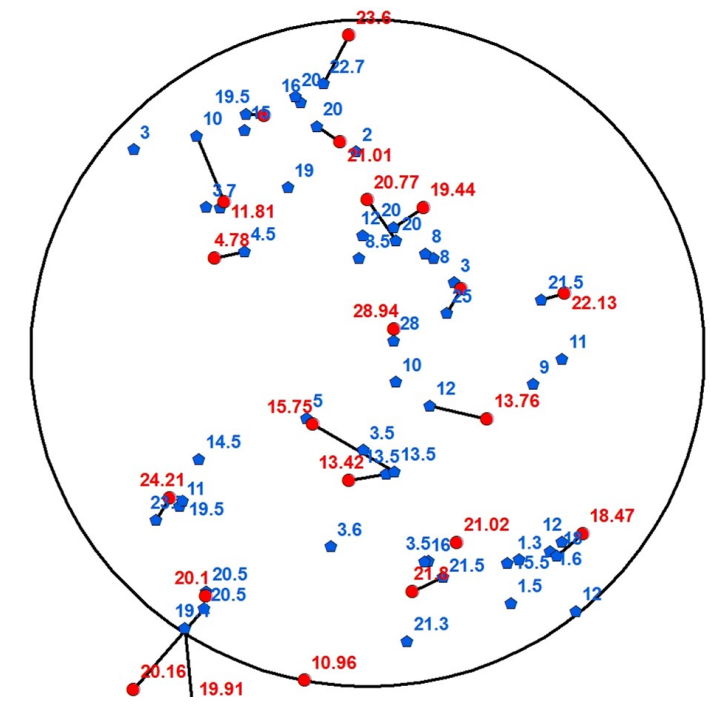

(a2) MCNCP-RNC

Fig. 3.2 Examples of MCNCP-RNC tree delineation for a plot in the Italian dataset (plot 77). Results are projected onto a 2D plane: each delineated tree is shown in red circles, and the locations of the ground truth are shown in blue pentagons. The dark circle (solid line) shows the $15 \mathrm{~m}$ radii field plot, where ground truth were measured. The dark solid line shows matching of segmented trees and ground truth. The numbers in red and blue colours indicate tree heights of segmented trees and ground truth, respectively.

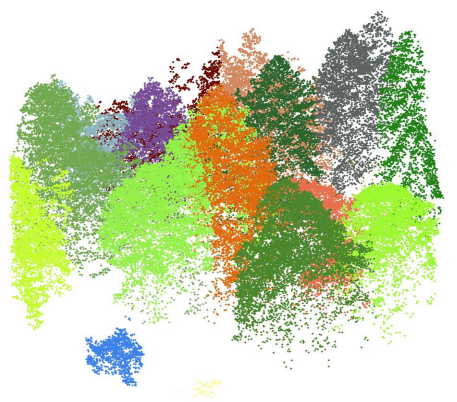

(a1) RNC

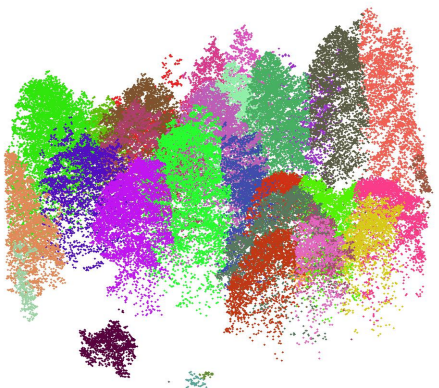

(a2) MCNCP-RNC

Fig. 3.3 3D examples of individual tree delineation by RNC (left) and MCNCP-RNC (right) algorithms in the Italian dataset (plot 77).

RNC detected correctly only $14 \%$ (7 out of 50 trees) ITCs while MCNCP-RNC $34 \%$ (17 trees out of 50 trees) ITCs in plot 77 of the Italian dataset. Moreover, it can be seen in 
Figure 3.3 that RNC leads to unrealistic tree delineation. The performance of RNC was poor in all the experiments we performed (results not shown), therefore we did not consider it further. The performances of TIFFS and MCNCP-RNC in the Italian dataset are shown in Tables 3.2 and 3.3. MCNCP-RNC showed slightly better performance to find understory trees compared to TIFFS. MCNCP-RNC algorithm outperformed TIFFS in five sites and the performance was the same in the other two test sites. Table 3.3 shows the performances of the ITC delineation algorithms at different tree height intervals in the Italian dataset. Both TIFFS and MCNCP-RNC showed poor performances to detect understory trees, even if MCNCP-RNC found a few more understory trees than TIFFS.

Table 3.2 The performance of delineation algorithms when applied to seven forest plots in the Italian datasets. Inside the bracket the method used to obtain local maxima is shown. 'Ground Truth' is the number of stems $(>1 \mathrm{~cm} \mathrm{DBH})$ recorded in the field plots. 'Extracted' means the number of trees delineated by the algorithms. while 'matched' indicates the number of correctly segmented trees, assessed by the NewFor matching algorithm.

\begin{tabular}{|c|c|c|c|c|c|c|c|c|c|}
\hline \multirow{2}{*}{ Plot } & \multirow{2}{*}{$\begin{array}{c}\text { Ground } \\
\text { Truth }\end{array}$} & \multicolumn{2}{|c|}{ MCNCP-RNC (MWF) } & \multicolumn{2}{|c|}{ TIFFS } & \multicolumn{2}{|c|}{ MCNCP-RNC (TIFFS) } & \multicolumn{2}{|c|}{ MCNCP-RNC (TIFFS) Hyp } \\
\hline & & Extracted & Matched & Extracted & Matched & Extracted & Matched & Extracted & Matched \\
\hline Plot 77 & 50 & 15 & 15 & 17 & 15 & 19 & 17 & 18 & 16 \\
\hline Plot 91 & 72 & 23 & 22 & 25 & 22 & 25 & 22 & 25 & 22 \\
\hline Plot 102 & 35 & 18 & 17 & 17 & 16 & 20 & 18 & 19 & 18 \\
\hline Plot 129 & 11 & 10 & 9 & 14 & 8 & 14 & 9 & 15 & 9 \\
\hline Plot 220 & 21 & 17 & 17 & 18 & 17 & 19 & 18 & 19 & 18 \\
\hline Plot 274 & 57 & 31 & 31 & 32 & 32 & 32 & 32 & 32 & 32 \\
\hline Plot 292 & 39 & 15 & 6 & 12 & 10 & 12 & 11 & 14 & 11 \\
\hline Overall & 285 & 129 & 117 & 135 & 120 & 141 & 127 & 142 & 126 \\
\hline
\end{tabular}

Table 3.3 The summary of the performance of delineation algorithms in the Italian dataset at different height scales. 'Extract' means the number of trees delineated by the algorithms. 'Match' is the number of trees that were matched to trees in seven mapped forest plots which had similar $(\mathrm{x}, \mathrm{y})$ coordinates and were of similar heights.

\begin{tabular}{|c|c|c|c|c|c|c|c|c|c|}
\hline \multirow{2}{*}{ Plot } & \multirow{2}{*}{$\begin{array}{c}\text { Ground } \\
\text { Truth }\end{array}$} & \multicolumn{2}{|c|}{ MCNCP-RNC (MWF) } & \multicolumn{2}{|c|}{ TIFFS } & \multicolumn{2}{|c|}{ MCNCP-RNC (TIFFS) } & \multicolumn{2}{|c|}{ MCNCP-RNC (TIFFS) Hyp } \\
\hline & & Extracted & Matched & Extracted & Matched & Extracted & Matched & Extracted & Matched \\
\hline$h \geq 20 \mathrm{~m}$ & 137 & 114 & 103 & 120 & 108 & 125 & 111 & 125 & 111 \\
\hline $15 \mathrm{~m} \leq h<20 \mathrm{~m}$ & 29 & 7 & 6 & 8 & 7 & 8 & 8 & 8 & 7 \\
\hline $10 \mathrm{~m} \leq h<15 \mathrm{~m}$ & 33 & 5 & 5 & 4 & 3 & 6 & 6 & 7 & 6 \\
\hline $5 \mathrm{~m} \leq h<10 \mathrm{~m}$ & 36 & 3 & 3 & 2 & 2 & 2 & 2 & 2 & 2 \\
\hline $2 \mathrm{~m} \leq h<5 \mathrm{~m}$ & 24 & 0 & 0 & 0 & 0 & 0 & 0 & 0 & 0 \\
\hline Overall & 285 & 129 & 117 & 135 & 120 & 141 & 127 & 142 & 126 \\
\hline
\end{tabular}


3D individual tree segmentation of integrated airborne LiDAR and optical imagery using normalised cut with priors

The evaluation of the MCNCP-RNC algorithm (with TIFFS-detected tree top positions as priors) was conducted using the NewFor benchmark dataset. The initial tree delineation provided by TIFFS was improved upon by the MCNCP-RNC segmentation in eight out of fourteen test sites (Table 3.4). In test sites 1, 5, 6, 7, 9, 10, 12 and 16, MCNCP-RNC extracted less trees and matched more trees. MCNCP-RNC performed as good as TIFFS in test sites $8,10,11,13$ and 18. In case of test site 17, the performance of MCNCP-RNC was particularly bad, as it matched five trees less compared to TIFFS. This was because a few tree crowns were so closely located each other, such that MCNCP-RNC merged them into a single tree crown. The performances were similar in the remaining five sites. Table 3.5 shows the performance of TIFFS and MCNCP-RNC algorithms in different height intervals. The table shows that oversegmentation occurred for segmenting trees above $20 \mathrm{~m}$ height in both TIFFS and MCNCP-RNC. Overall, MCNCP-RNC reduced the false tree detections, and increased the number of trees correctly assigned. MCNCP-RNC also showed a marginal improvement for finding small trees. Considering that we used a fixed set of parameters for all benchmark testing, the performance of MCNCP-RNC could probably be improved with manual parameter tuning. Figure 3.4 illustrates the segmentation of trees in study areas 7 and 16 using MCNCP-RNC.

Both TIFFS and MCNCP-RNC were less successful at delineating trees within the English dataset, but MCNCP-RNC outperformed TIFFS. Broadleaf trees have less distinctive tree tops than the conifers of Italian and Alpine datasets, making delineation more of a challenge. Approximately 8-10 percent of trees were successfully delineated, but it is reasonable because most trees omitted by algorithms were small trees of under $15 \mathrm{~m}$ height. Table 3.6 shows the number of extracted and correctly delineated trees. The first row of the table shows the overall accuracy and the second row shows the canopy crown detection results. The numbers inside the brackets in the second row describes the number of correctly matched trees with ground truth heights of over 20m. Overall, MCNCP-RNC extracted 346 
Table 3.4 The NewFor benchmark test of TIFFS and MCNCP-RNC. 'Extract' means the number of trees delineated by the algorithms. 'Match' defines the number of correctly assigned trees.

\begin{tabular}{c||c|c|c|c|c}
\hline \multirow{2}{*}{$\begin{array}{c}\text { Study } \\
\text { area }\end{array}$} & Ground & \multicolumn{2}{|c|}{ TIFFS } & \multicolumn{2}{c}{ MCNCP-RNC (TIFFS) } \\
\cline { 3 - 6 } & truth & Extract & Match & Extract & Match \\
\hline \hline 01 & 352 & 358 & 181 & 351 & 188 \\
\hline 05 & 235 & 45 & 41 & 47 & 44 \\
\hline 06 & 47 & 32 & 28 & 34 & 30 \\
\hline 07 & 79 & 61 & 52 & 57 & 53 \\
\hline 08 & 107 & 43 & 39 & 44 & 38 \\
\hline 09 & 169 & 71 & 58 & 67 & 60 \\
\hline 10 & 106 & 79 & 40 & 82 & 42 \\
\hline 11 & 22 & 15 & 10 & 14 & 10 \\
\hline 12 & 49 & 83 & 31 & 76 & 32 \\
\hline 13 & 100 & 63 & 45 & 62 & 45 \\
\hline 15 & 53 & 42 & 24 & 41 & 23 \\
\hline 16 & 37 & 45 & 21 & 42 & 23 \\
\hline 17 & 117 & 82 & 69 & 80 & 64 \\
\hline 18 & 92 & 58 & 42 & 62 & 42 \\
\hline \hline Overall & 1565 & 1074 & 681 & 1060 & 695 \\
\hline
\end{tabular}

Table 3.5 The summary of the performance of delineation algorithms in the NewFor benchmark dataset in different tree height tiers. 'Extract' means the number of trees delineated by the algorithms. 'Match' is the number of trees that were matched to trees in the mapped forest plot which had similar $(\mathrm{x}, \mathrm{y})$ coordinates and were of similar heights

\begin{tabular}{c||c|c|c|c|c}
\hline \multirow{2}{*}{$\begin{array}{c}\text { Study } \\
\text { area }\end{array}$} & Ground & \multicolumn{2}{|c|}{ TIFFS } & \multicolumn{2}{c}{ MCNCP-RNC (TIFFS) } \\
\cline { 3 - 6 } & truth & Extract & Match & Extract & Match \\
\hline \hline$h \geq 20 \mathrm{~m}$ & 638 & 811 & 547 & 797 & 550 \\
\hline $15 \mathrm{~m} \leq h<20 \mathrm{~m}$ & 279 & 147 & 96 & 155 & 97 \\
\hline $10 \mathrm{~m} \leq h<15 \mathrm{~m}$ & 292 & 34 & 21 & 40 & 27 \\
\hline $5 \mathrm{~m} \leq h<10 \mathrm{~m}$ & 270 & 41 & 14 & 41 & 18 \\
\hline $2 \mathrm{~m} \leq h<5 \mathrm{~m}$ & 86 & 41 & 3 & 27 & 3 \\
\hline \hline Overall & 1565 & 1074 & 681 & 1060 & 695 \\
\hline
\end{tabular}

trees and correctly matched 197 trees, showing that 14 more trees were correctly segmented than that by TIFFS. Due to the low point density of this dataset, it is very challenging to find small trees, which cause huge errors in the overall segmentation accuracy. Therefore, we also evaluated the delineation accuracy of ITCs over different height intervals (see Table 3.6). With respect to the detection accuracy, TIFFS found 141 trees while MCNCP-RNC detected 147. The analysis shows that both TIFFS and MCNCP-RNC oversegmented ITCs. However, 


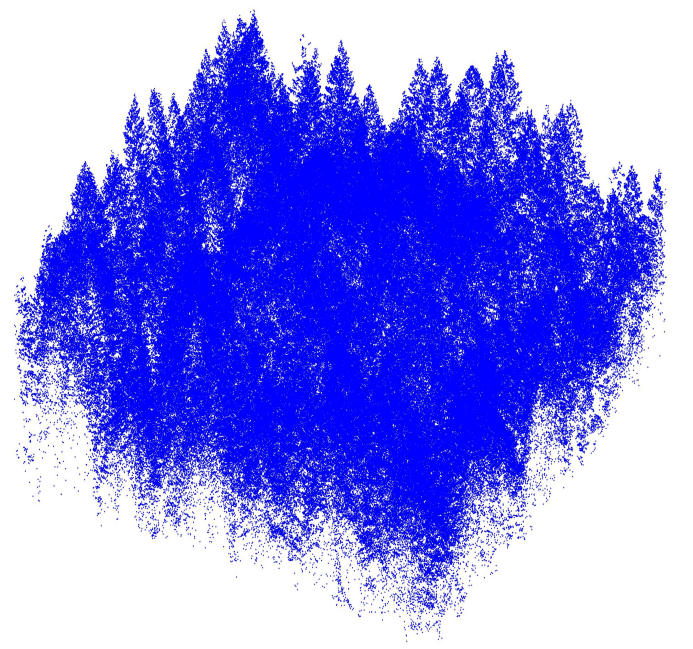

(a) Site 7

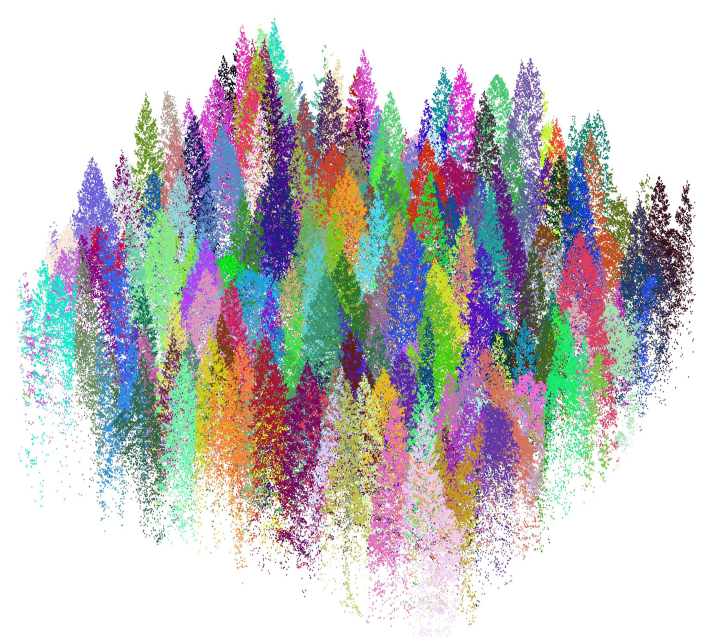

(c) MCNCP-RNC

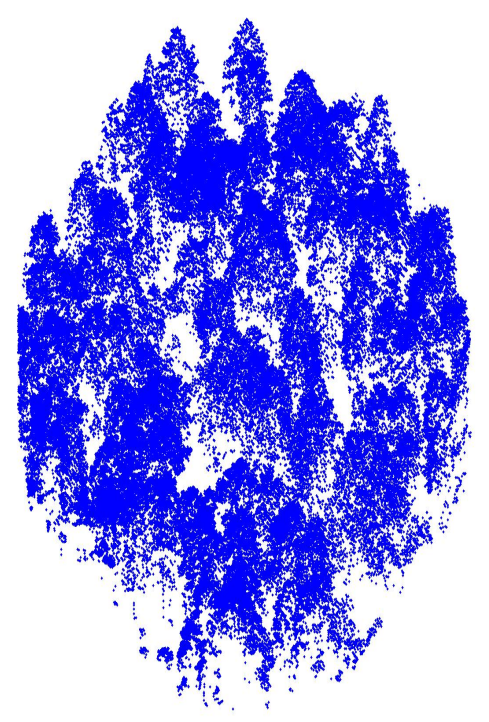

(b) Site 16

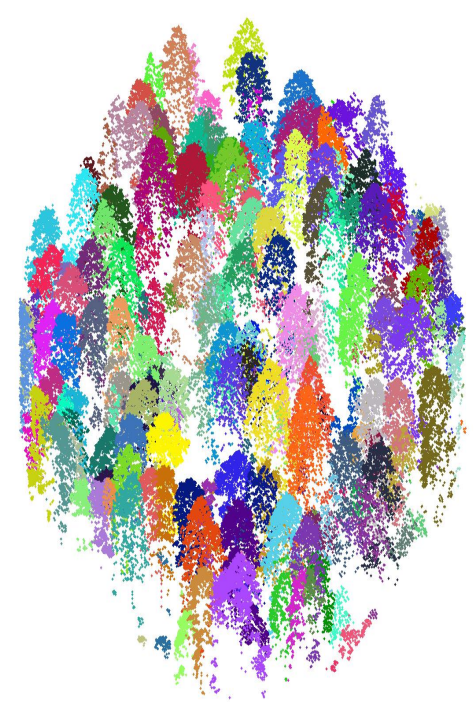

(d) MCNCP-RNC

Fig. 3.4 Examples of MCNCP-RNC segmentation of the NEWFOR benchmark datasets. The first row shows the LiDAR point clouds from test sites 7 (Left) and 16 (Right). The second row presents the results of our ITC delineation method.

the ratio between extract/match canopy trees was $50.3 \%$ and $54.8 \%$, respectively. False 
positives were reduced by $4.5 \%$ in MCNCP-RNC. Therefore, MCNCP-RNC outperformed TIFFS in both overall and canopy crown segmentation cases.

Table 3.7 compares the computational time for the RNC and MCNCP-RNC with TIFFS, when applied to the Italian datasets (plots of $15 \mathrm{~m}$ radius with unusually high point density). As RNC needs to construct a graph recursively to segment trees, its computational cost is more expensive than that of TIFFS or MCNCP-RNC on this high point-density dataset. RNC was ten times slower than MCNCP and twice slower than MCNCP-RNC. This is because RNC can separate point clouds into only two clusters at each step, and constructing a large graph and solving eigensystem repeatedly cost computational time. MCNCP-RNC, however, separates LiDAR point cloud into ITCs during the MCNCP step, so the graph size of each segment is relatively small. TIFFS is faster than RNC, MCNCP or MCNCP-RNC because it relies only on the CHM.

Table 3.6 The performance of the delineation algorithms in the English dataset, by height tier. 'Extract' means the number of delineated trees. 'Match' is the number of trees that were matched to trees in the mapped forest plot which had similar $(\mathrm{x}, \mathrm{y})$ coordinates and similar heights. In the first column, the range of heights in each tier is shown.

\begin{tabular}{c||c|c|c|c|c|c|c}
\hline \multirow{2}{*}{$\begin{array}{c}\text { Study area: } \\
\text { Wytham }\end{array}$} & Ground & \multicolumn{2}{|c|}{ TIFFS } & \multicolumn{2}{c|}{ MCNCP-RNC (TIFFS) } & \multicolumn{2}{c}{ MCNCP-RNC (TIFFS) Hyp } \\
\cline { 3 - 8 } & truth & Extract & Match & Extract & Match & Extract & Match \\
\hline \hline$h>0 \mathrm{~m}$ & 2116 & 342 & 183 & 346 & 197 & 419 & 225 \\
\hline \hline$h \geq 20 \mathrm{~m}$ & 194 & 280 & 141 & 264 & 147 & 318 & 166 \\
\hline $15 \mathrm{~m} \leq h<20 \mathrm{~m}$ & 476 & 61 & 41 & 76 & 50 & 96 & 58 \\
\hline $10 \mathrm{~m} \leq h<15 \mathrm{~m}$ & 523 & 1 & 1 & 4 & 0 & 3 & 1 \\
\hline $5 \mathrm{~m} \leq h<10 \mathrm{~m}$ & 756 & 0 & 0 & 2 & 0 & 2 & 0 \\
\hline $2 \mathrm{~m} \leq h<5 \mathrm{~m}$ & 159 & 0 & 0 & 0 & 0 & 0 & 0 \\
\hline
\end{tabular}

\subsubsection{Tree delineation from LiDAR and hyperspectral imagery}

MCNCP-RNC provides a framework for using both LiDAR point cloud and features from hyperspectral imagery, and we tested this approach with the Italian and English datasets. For the Italian dataset (Tables 3.2 and 3.3) hyperspectral imagery does not improve the already excellent segmentation of upper canopy trees. In plot 77, 16 out of 18 trees were correctly 
3D individual tree segmentation of integrated airborne LiDAR and optical imagery using 76 normalised cut with priors

matched compared to 17 out of 19 trees in the LiDAR-only analysis. In plot 102, fewer false positive were detected than the LiDAR-only analysis, while more false positive were detected in plots 129 and 292. No difference was noticed in plots 91, 220 and 274. In the case of the English dataset, performances were slightly improved (Table 3.6). Only $8 \sim 10 \%$ of the trees were correctly delineated. MCNCP-RNC with LiDAR and hyperspectral imagery detected significantly more trees in the English dataset: it detected 42 ITCs more than TIFFS algorithm (although the ratio between extracted trees and matched trees were similar) and more trees than using LiDAR data only. As there are large numbers of small trees in the dataset, we examined only upper canopy trees over $20 \mathrm{~m}$ height ( $9 \%$ of recorded trees): in this case the number of extracted and correctly assigned trees increased by 38 and 19, respectively. However, we observed that large trees were oversegmented. Canopy trees over $20 \mathrm{~m}$ were 194 in the ground truth data, while MCNCP-RNC extracted 318 trees, out of which 166 trees were correctly matched. The ratio of extracted to matched canopy trees over $20 \mathrm{~m}$ for TIFFS, MCNCP-RNC with LiDAR only and MCNCP-RNC with LiDAR and hyperspectral imagery was $50.3 \%, 54.8 \%$ and $52.2 \%$, respectively. So more trees were detected but at the expense of more false positives. The detection of the understory trees $(<15 \mathrm{~m})$ in Table 3.6 was poor for all algorithms. Specifically, MCNCP-RNC with both LiDAR and hyperspectral imagery found 8 more trees than MCNCP-RNC with LiDAR only and 17 more trees than TIFFS in the range of tree heights in $10 \mathrm{~m}<x<15 \mathrm{~m}$ at the expense of a larger commission error.

Table 3.7 Computation time in seconds of RNC, MCNC, TIFFS and MCNCP-RNC applied to the Italian dataset.

\begin{tabular}{c||c|c|c|c}
\hline & RNC & MCNC & MCNCP-RNC & TIFFS \\
\hline \hline Plot 77 & 486 & 39 & 227 & 12 \\
\hline Plot 91 & 621 & 57 & 227 & 12 \\
\hline plot 102 & 888 & 79 & 494 & 19 \\
\hline plot 129 & 417 & 48 & 276 & 12 \\
\hline plot 220 & 1085 & 103 & 809 & 14 \\
\hline plot 274 & 686 & 84 & 320 & 12 \\
\hline plot 292 & 653 & 52 & 377 & 12 \\
\hline
\end{tabular}




\subsection{Discussion}

\subsubsection{The application of graph cut approaches to tree delineation}

This paper proposes a tree delineation technique based on a normalised graph cut framework, which can make use of LiDAR and any optical imagery. We included an image registration step to ensure co-alignment between images, and a novel feature reduction step to find a meaningful set of features from high dimensional datasets. The RNC method failed to deliver accurate results, perhaps because it discards too much useful information by working with only the second smallest eigenvector $[237,256]$, whereas the proposed multi-class cut approach, constrained with information from classical CHM-based delineation, improved the quality of tree top matching significantly. The validity of MCNCP-RNC has been demonstrated by experiments using the NewFor benchmark, English and Italian datasets, outperforming a leading CHM-based segmentation algorithm in most cases.

Priors strongly influence the tree detection accuracy of MCNCP-RNC. The algorithm can successfully detect more trees than predicted by the number of local maxima as RNC step checks the further separability of each cluster. However, MCNCP-RNC shows limited performance at merging clusters identified in the prior because our method is strongly constrained by local maxima priors. For example, TIFFS incorrectly detected four tree tops in plot 129 of the Italian dataset, while MWF missed only one tree top. This leads to MCNCP-RNC with MWF outperforming MCNCP-RNC with TIFFS in the plot 129. Graph cut could still play a role in merging oversegmented trees together in a limited condition. In the English dataset, TIFFS generated false tree tops. In theory, MCNC-RNC should extract more, or the same number of trees than TIFFS. The result showed that MCNCP-RNC reduced the number of extracted trees by 14 compared to TIFFS. This is probably because TIFFS priors are very close to each other. In this case, these prior clusters are so similar that MCNCP-RNC can merge the clusters. One solution of this problem is imposing a "soft" 
3D individual tree segmentation of integrated airborne LiDAR and optical imagery using 78 normalised cut with priors

factor to the correlation constraint. In the "soft" case, the correlation constraint need not be satisfied; instead the algorithm finds the balance between the maximal correlation and optimal normalised cut separation. This would require us to build a new optimisation model, which is beyond the scope of this study.

\subsubsection{Combining LiDAR and hyperspectral imagery to improve delin- eation}

MCNCP-RNC was able to detect more understory trees than CHM-based approached, but could not find the small trees under dense forests. In principle, it should be able to detect understory trees if the LiDAR device specifications (e.g. point density, number of returns recorded per pulse) was good enough to represent understory structures. In case of the English dataset, the point density was only 6 points per $\mathrm{m}^{2}$, making it impossible to find any understory structure. In contrast, the LiDAR point density of the Italian dataset was so high that internal structures of trees, and understory trees can be identified, which may explain why MCNCP-RNC performed better than TIFFS. However, even with this dataset there were still a number of understory trees undetected by MCNCP-RNC. If we consider vertical LiDAR point profiles of each canopy, we can change the parameters for RNC step. Duncanson et al. [86] used the vertical distributions of LiDAR point clouds to separate understory trees. After an initial ITC delineation using a watershed algorithm the authors examined the vertical LiDAR point distribution to see whether it showed continuous decrease from the top canopy or subsequent continuous increase in the middle or low height. If the vertical point distribution showed subsequent increase in the middle or low height, then these parts were separated as understory trees. This approach can be applied to our segmentation algorithm directly or the vertical profiles can be parameterised to be incorporated into the RNC step. Also full-waveform LiDAR may provide an opportunity to find internal structures in more details. Reitberger et al. [224] used RNC with full-waveform LiDAR, which had 9 points 
per $\mathrm{m}^{2}$. They suggested full-waveform LiDAR pulse and intensity with calibration could help to detect ITCs in the understory. Unfortunately, LiDAR intensity was not calibrated in our datasets due to an automatic gain control system on the Leica instrument, which regulates LiDAR intensities in non-linear and opaque way, so intensity could not be used in the segmentation.

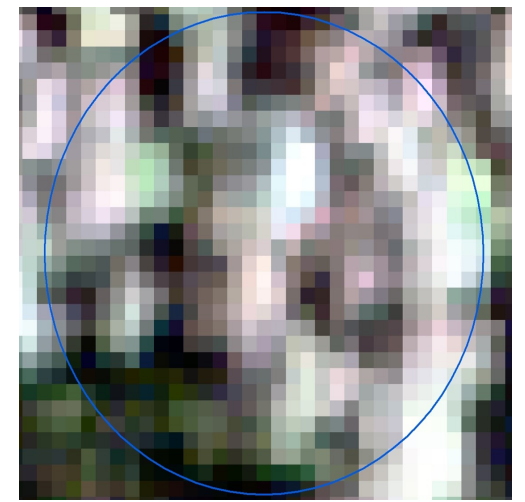

(a) Hyperspectral imagery of the Italian dataset (plot 220)

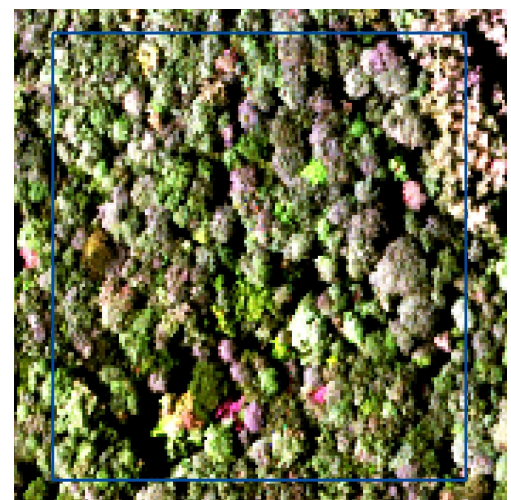

(b) Hyperspectral imagery of the English dataset

Fig. 3.5 Examples of hyperspectral images in the Italian and English datasets. The blue circle and square represent the size of test sites of the Italian $\left(706 \mathrm{~m}^{2}\right)$ and English datasets (18 ha), respectively.

Figure 3.5 shows the hyperspectral images of the Italian and English datasets. The experiments of MCNC-RNC with both LiDAR and hyperspectral data in the Italian dataset showed that the ITC delineation was not influenced by hyperspectral imagery, while those of the English dataset improved the number of trees correctly segmented at the expense of more oversegmentation. The poor results in the Italian dataset might be related to the high LiDAR point density compared to the hyperspectral information. As shown in Figure 3.5(a), the pixel size of the hyperspectral imagery in the Italian dataset was too large to give precise feature information to segment dense LiDAR point clouds ( $\geq 80$ points per $\mathrm{m}^{2}$ ). LiDAR point density was very high, i.e. almost hundred points were represented by a single hyperspectral pixel. Under this condition, ITC delineation is mainly driven by the LiDAR point cloud rather than hyperspectral imagery. As in the Italian dataset there were 
3D individual tree segmentation of integrated airborne LiDAR and optical imagery using 80 normalised cut with priors

often only two dominant species, the information provided by the hyperspectral imagery was not useful for the ITC delineation. In the English dataset, on the other hand, LiDAR point density was low ( 6 points per $\mathrm{m}^{2}$ ) and there was a higher species diversity (see Figure 3.5(b)). These two conditions made the English dataset ideal for ITC delineation using both LiDAR and hyperspectral imagery. However, it was also noticed that more false positive were observed for MCNC-RNC with both LiDAR and hyperspectral imagery. This may be related to shade effects or registration errors remaining in the hyperspectral imagery. It was reported that the illumination effects contained in the first principal component of the hyperspectral imagery cause inaccurate ITC delineation [250], so we extracted 2-5th principal components of hyperspectral imagery for ITC delineation. However, the illumination effects may still remain in the principal components we used for the delineation [250].

\subsubsection{The problem of detecting understory trees}

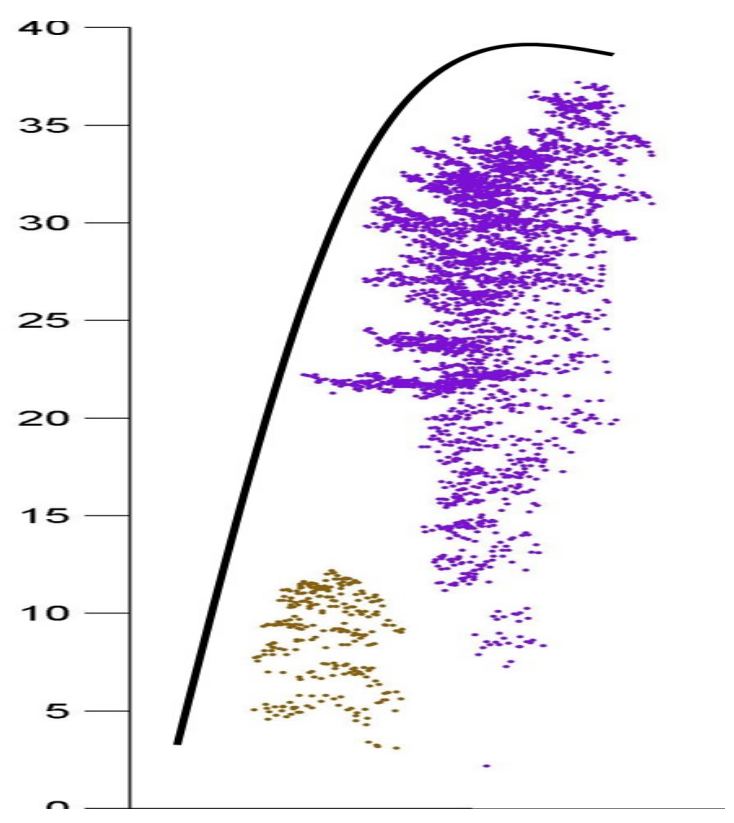

Fig. 3.6 Example of MCNCP-RNC segmentation of understory tree in the Italian dataset. The black solid line is the interpolation line $(\mathrm{CHM})$ of the LiDAR point cloud. Point clouds in sienna and purple colours are the segmented ITCs using MCNCP-RNC. 
The detection of understory trees is strongly influenced by the point density. In the English dataset, the relatively low point density ( 6 points per $\mathrm{m}^{2}$ ) made it was impossible to detect understory trees, thus causing very low detection rates. Low detection rate may also be attributable to uncertainties in the locations of trees on the ground, which meant that matches were not made by the NewFor algorithm even though delineation had been accurate. On the opposite in the case of the Italian dataset, where the point density was extremely high, it was possible to extract understory trees. Indeed the performance improvement of the MCNCP-RNC with respect to TIFFS on this dataset was partially because it found more understory trees. Figure 3.6 shows an example of understory tree delineation using MCNCP-RNC. In this example, in the CHM (black solid line) only a single tree crown was visible, as the CHM is constructed by the interpolation of the LiDAR point cloud. In contrast, MCNCP-RNC can delineate two ITCs in Figure 3.6 because the RNC process checks further separability of each ITC and the LiDAR point density was high enough to find understory structures. In the example of Figure 3.6, the understory tree was clearly separable because there was a gap between trees. However, this is not common in dense LiDAR point clouds because canopy and understory trees usually overlap. In this case, parameters for graph weights should be chosen carefully, otherwise MCNCP-RNC fails to delineate ITCs. Since we fixed the parameters for the RNC process for all the ITCs, it is hard to delineate subcanopy trees efficiently. LiDAR vertical profiles of each canopy tree may provide good statistics for separating understory trees [86]. If we can learn parameters automatically from LiDAR vertical statistics, then ITC delineation can be extended to find understory trees. However, analysing LiDAR vertical profiles and learning ITC parameters are beyond the scope of our research, so we leave them for future work. 
3D individual tree segmentation of integrated airborne LiDAR and optical imagery using

\subsubsection{Concluding remarks}

This paper developed a new normalised cut based ITC delineation method, called MCNCPRNC. The performance of MCNCP-RNC was compared with TIFFS in three different datasets: the Italian, NewFor benchmark and English datasets. Our experiments showed that MCNCP-RNC outperforms TIFFS in all test datasets. MCNCP-RNC easily incorporated optical imagery alongside LiDAR data, so ITC delineation could be conducted using LiDAR and optical imagery. This multi-sensor approach could help ITC delineation. Since MCNCPRNC algorithm delineated ITCs directly from the 3D point cloud, it could find a few understory trees. Since MCNCP-RNC can detect more trees with accurate parameters than conventional CHM-based methods, it can provide better understanding of ITC characteristics.

Although MCNCP-RNC showed superior performances compared with classic CHMbased approaches, there are a number points that could be improved. TIFFS algorithm detected many false tree tops. A problem is that MCNCP-RNC is significantly influenced by these false tree tops, which are regarded as prior information. MCNCP-RNC can only merge when two tree locations in the priors are very close together. Replacing our hard constraint with a softer one may resolve this problem. MCNCP-RNC is still computationally expensive when we need to compute too many eigenvectors, although MCNCP-RNC was computationally faster than RNC. For example, if we have thousands of local maxima, then we need to compute the first thousands of eigenvectors to delineate the ITCs. Since these eigenvectors are usually dense, it increases the memory complexity. In order to avoid this problem, a domain decomposition technique needs to be implemented. In theory, understory trees can be detected by RNC process if the LiDAR point density is high enough. This can be resolved by checking LiDAR vertical profiles statistics of each canopy tree [86]. This method may provide a useful parameter to guide RNC algorithm to delineate understory trees. Full-waveform LiDAR can also improve ITC segmentation as it could provide more details about the internal structure. 


\section{Chapter 4}

\section{Individual tree species classification from airborne multi-sensor imagery}

\subsection{Introduction}

Having maps of individual tree locations is fundamental to understanding forest responses to global change, providing a basis for monitoring species distribution patterns, responses to stress, disease and exotic-species spread and deforestation [14]. Mapping species using conventional surveying methods require a large amount of time and effort so few tree maps extend beyond 50-ha (50 hectares) in extent; larger scale maps have been generated by sampling in small plots distributed over wider regions and interpolating [132]. The development of sophisticated remote sensing technologies is making it increasingly feasible to monitor single trees in forests using aircraft or satellites. In particular, some airborne hyperspectral sensors can measure the radiance of a target in narrow bands spread from visible to short-wave infrared wavelengths of the electromagnetic spectrum (400-2500nm). Studies carried on with hand-held spectrometers show that species are often distinguishable from their leaf spectra, even in diverse tropical forests. For example, about half of 188 species sampled from a humid tropical forest in Hawaii could be distinguished from their 
spectra, with differential reflectance in the SWIR as well as the visible and NIR being important[91, 93]. Such results prognosticate the identification of individual trees by remote sensing with similar sensors $[7,69,54,15]$.

Airborne hyperspectral imaging provides spectral properties of the vegetation canopy at large scale, which can be used to identify tree species. Scaling-up species classification from leaf level to canopy level remains challenging as reflectance signals of vegetation canopy are influenced by spectral mixing, leaf density, leaf angle distribution, crown shape and shading [54]. Nevertheless, recent studies have successfully used hyperspectral imaging to map species in tropical forests [67, 269, 93], savanna woodlands [63], Mediterranean woodlands[77, 73], temperate deciduous forests [116, 126] and boreal forests [79]. Clark et al. [67] pioneered the use of hyperspectral data to identify canopy species in tropical rain forest, detecting seven tree species with 92\% accuracy. Cho et al. [71] detected ten tree species with 57\% accuracy in the lowveld woodlands of South Africa. Dalponte et al. [77] classified 23 species from two Mediterranean woodlands, and achieved $88 \%$ and $96 \%$ accuracy for those regions. Therefore, rapid advances are being made in this context.

The accuracy of pixel-level species maps can be improved by combining features from Light Detection And Ranging (LiDAR) and hyperspectral imagery in classification algorithms. LiDAR produces 3D point clouds indicating forest structures, from which canopy height and various other metrics can be extracted for each pixel. Features comprised of this structural information complement the optical data provided by hyperspectral sensors, particularly as LiDAR data is not influenced by illumination artifacts such as shading of shorter trees by their taller neighbours [74]. High species classification accuracy (89\%) in an Italian temperate floodplain forest was achieved fusing LiDAR and hyperspectral imagery before classification [74]. Jones et al. [146] showed that LiDAR and hyperspectral fusion can improve species classification in a mixed broadleaf-conifer forest. The work in [76] also showed that improvement was observed when LiDAR and hyperspectral imagery were 
used together in alpine forests containing a mixture of beech and conifers. However, the importance of LiDAR-derived features on classification success varies greatly among species $[146,76,108]$.

The approaches described above illustrate the advances made in pixel-based classification, but less progress has been made in mapping individual tree crowns (ITCs) using multi-sensor techniques. The 3D point clouds provided by LiDAR provide an excellent data for ITC delineation $[60,74,146,76,108]$, while canopy spectral information can be obtained from the corresponding pixels of hyperspectral imagery within each of the identified crowns, so in principle this combination of information is powerful [71, 122, 268, 257, 3, 78]. Mapping species at single tree scale has been demonstrated in urban environments, where trees are sparsely distributed [257, 268, 3]. For example, Alonzo et al. [3] mapped 30 urban tree species at ITC-level using full spectral bands of hyperspectral imagery and seven tree structural parameters derived from LiDAR. However, for more complex environments, we know of only three studies that have investigated ITC-level species classification using a multi-sensor approach: Colgan et al. delineated ITCs from LiDAR and classified species from hyperspectral imagery in a savanna woodland, then combined these results [71]. Heinzel and Koch showed that under-segmentation of ITCs using LiDAR-based delineation could be rectified by using species classification information alongside LiDAR [122]. Dalponte et al. improved species classification by selecting pixels inside of ITCs for training species for the classification and excluding all others [78].

This paper develops a generic workflow for tree species classification at ITC-level from LiDAR and hyperspectral sensors. We deal with several technical challenges:

- Multi-sensor imaging requires images recorded by various sensors to be co-aligned, but different sensor characteristics result in scale, rotation or translation mismatches between images, making correction a pre-requisite. Our workflow includes a image 
registration step using the NGF-Curv method in Chapter 2 to co-align hyperspectral imagery and LiDAR.

- Locating ITCs in the 3D LiDAR point cloud or optical imagery requires an accurate tree delineation algorithm, but most established approaches are inaccurate in broadleaf forests [217][60]. Our workflow includes a normalised graph cut scheme in Chapter 3 to delineate ITCs using LiDAR 3D point cloud information alongside optical imagery.

- When selecting features to use in classification it is recognised that the dimensionality of hyperspectral data must be reduced to improve computationally efficiency, but hyperspectral imagery may have a significant noise component so we use robust PCA (rPCA) to reduce dimensionality and strip away some of that noise [53].

- Finally, tree species classification at both pixel-level and ITC-level with support vector machine (SVM) [55] is implemented.

The main contribution of this paper is the development of a systematic workflow to map tree species at both pixel-level and ITC-level from multi-sensor imagery utilizing a combination of new and established approaches, which provide a powerful new approach for tree mapping. We test the efficacy of this approach by working with airborne imagery collected over a 18-ha mapped stand of temperate woodland in the UK. Historically managed temperate forests are recognised as being particularly difficult for ITC delineation because they have relatively even upper canopies comprised of intercalated crowns. To the best of our knowledge, only a single study has explored ITC-level species classification in a temperate forest [122].

The paper is organized as follows: in Section 4.2, we introduce the temperate forest datasets tested in this paper. In Section 4.3, we present our workflow for tree species classification at both pixel-level and ITC-level from LiDAR and hyperspectral sensors. The 
results and discussion are shown in Section 4.4. Conclusions and outlook are given in Section 4.5 .

\subsection{Data description}

\subsubsection{Study site and field data}

We used the English dataset explained in section 3.4, Chapter 3. As subcanopy species and shrubs are hard to detect by remote sensing, this study focuses on mapping the six most common canopy tree species listed in Table 4.1. As shown in Chapter 3, tree heights were estimated by species-specific allometric equations. We arbitrarily labeled trees $>18 \mathrm{~m}$ height as "canopy trees" (Table 4.1) and used these to assess the accuracy of species detection.

Table 4.1 The numbers of individual and canopy trees of six species recorded in the English dataset

\begin{tabular}{l|l|c|c}
\hline Species & Common name & $\begin{array}{c}\text { No. of } \\
\text { individual trees }\end{array}$ & $\begin{array}{c}\text { No. of } \\
\text { canopy trees }\end{array}$ \\
\hline \hline Fraxinus excelsior & European ash & 5346 & 1249 \\
\hline Acer pseudoplatanus & Sycamore & 7716 & 778 \\
\hline Larix decidua & European larch & 99 & 98 \\
\hline Quercus robur & English oak & 381 & 201 \\
\hline Fagus sylvatica & European beech & 195 & 4 \\
\hline Betula spp. & Birch & 85 & 16 \\
\hline
\end{tabular}

\subsubsection{Airborne survey}

As shown in the previous chapter, airborne surveying was conducted in the Wytham wood natural reserve on 24 June 2014 by NERC-ARSF. Hyperspectral imagery and LiDAR data were collected simultaneously. In this study, we did not apply atmospheric correction to the hyperspectral imagery. Although atmospheric correction is an important step to find the linkage between leaf chemistry and canopy spectral signatures, calculating reflectance from 
radiance involves multiplying radiance values of all pixels by the same correction factors, so it does not influence species classification $[127,74,78]$. A bi-directional distribution function (BRDF) correction was not applied since only a narrow strip from a single flight line was used, so illumination and sensor geometry are similar for all pixels. If the hyperspectral imagery had been obtained from several flight lines, radiometric normalisation would been needed, and this step would need to be added into our workflow Figure 4.1.

\subsection{Method}

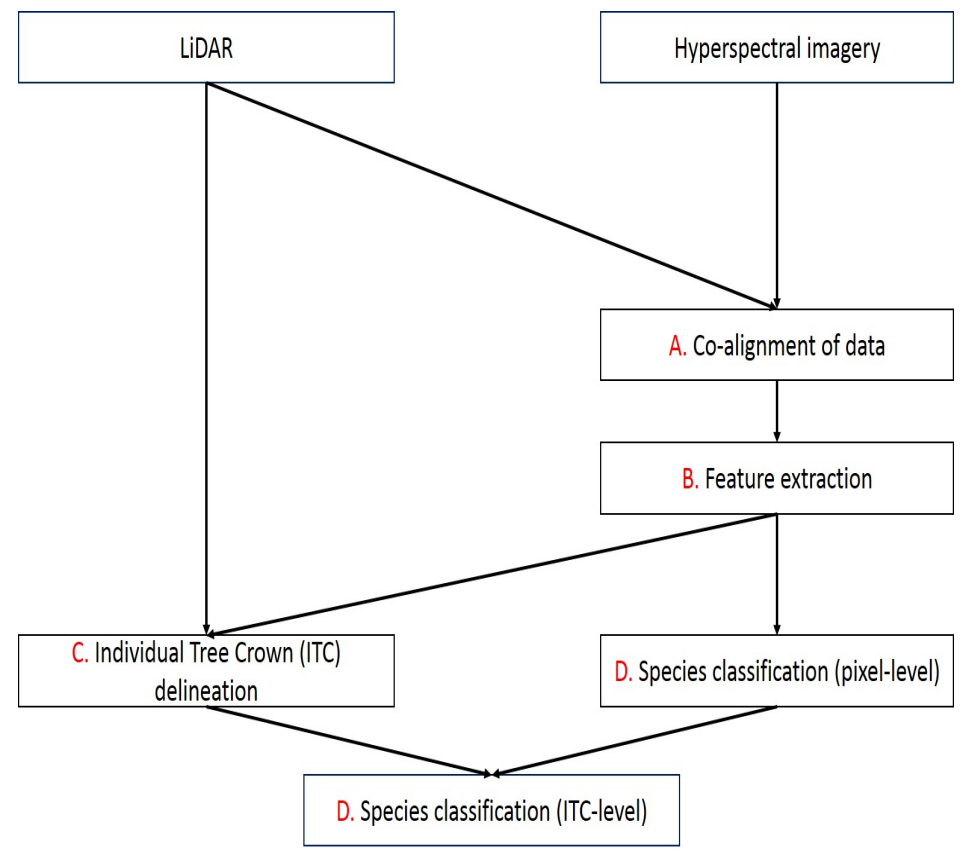

Fig. 4.1 Workflow used to detect individual tree crown and identify their species by fusing LiDAR and hyperspectral imagery.

In this section, we present our processing workflow for pixel-level and ITC-level tree species classification from LiDAR and hyperspectral imagery, see Figure 4.1. The coalignment step uses the NGF-Curv image registration method explained in Chapter 2, the feature extraction from hyperspectral imagery is carried out with the rPCA method [53], the ITC delineation step uses a LiDAR point cloud based clustering method also informed 
by hyperspectral information, MCNCP-RNC, and, finally, the tree species classification at pixel-level and ITC-level is conducted with a SVM classifier [55] with the majority voting rule over each delineated crown area. The workflow in Figure 4.1 is very general, and the methods are described in greater detail below.

\subsubsection{Co-alignment of LiDAR and hyperspectral imagery}

Co-alignment of LiDAR and hyperspectral imagery can be achieved by ground control points or by unsupervised image registration. We adopted NGF-Curv registration explained in Chapter 2. In this study, digital surface model (DSM) of LiDAR, which is the interpolation of LiDAR first returns, was used for registration with hyperspectral imagery.

\subsubsection{Feature extraction}

Extracting feature information from the hyperspectral imagery is a key step to map individual trees with species information. As hyperspectral imagery contains a high number of spectral bands, i.e. dimensionality is high, the meaningful features are hidden inside an enormous number of spectral bands. To extract some or whole meaningful features, a feature reduction technique rPCA described in Chapter 3 is implemented in our workflow. In this study, rPCA reduced 361 spectral bands of hyperspectral imagery to 20 principal components. These principal components were used to delineate ITCs alongside LiDAR and classify species.

\subsubsection{Individual tree crown delineation}

Individual tree crown delineation is performed by a normalised graphcut method constrained by prior knowledge directly on the 3D LiDAR point clouds and the extracted features from subsection 4.3.2. See method MCNCP-RNC in Chapter 3 for the detailed methodology of the algorithm. The locations of local maxima were computed by the toolbox for LiDAR 
data filtering and a standard forest analysis (TIFFS, Globalidar ltd.) [60]. Although we used TIFFS for extracting priors, users could use any tree top searching algorithm to get priors.

\subsubsection{Species classification}

Table 4.2 Number of pixels for each species of the training and test samples for tree species classification

\begin{tabular}{l|c|c}
\hline Species & $\begin{array}{c}\text { Training } \\
\text { samples }\end{array}$ & $\begin{array}{c}\text { Test } \\
\text { samples }\end{array}$ \\
\hline \hline Fraxinus excelsior & 284 & 458 \\
\hline Acer pseudoplatanus & 350 & 647 \\
\hline Larix decidua & 144 & 135 \\
\hline Quercus robur & 228 & 188 \\
\hline Fagus sylvatica & 133 & 192 \\
\hline Betula spp. & 106 & 105 \\
\hline Shade & 824 & 109 \\
\hline \hline Overall (NO. of pixels) & 2069 & 1834 \\
\hline
\end{tabular}

For the classification of the tree species we used the SVM method that is a non-parametric supervised classifier that has been showed to be superior to other classification strategies in several studies $[74,92,79,78]$. We applied SVM on the extracted features from subsection 4.3.2 in order to classify species, initially at the pixel level.

For a training data set $\mathscr{T}=\left\{\left(x_{1}, y_{1}\right), \cdots,\left(x_{n}, y_{n}\right)\right\}$ consisting of pairs of feature vectors $x_{i} \in \mathscr{F}$ (where $\mathscr{F} \subset \mathbb{R}^{m}$ is a $m$-dimensional feature space), and labels $y_{i}$ (for example the binary label $\left.y_{i} \in\{-1,1\}\right)$. SVM finds a separating hyperplane $\left.H:=\left\{x \mid\left\langle w, \eta\left(x_{i}\right)\right\rangle-b\right)=0\right\}$ (where $w$ is a normal vector to the hyperplane, $b$ is the intercept and $\eta\left(x_{i}\right): \mathscr{F} \rightarrow \overline{\mathscr{F}}$ is a non-linear embedding that transforms the original feature space to a higher dimensional space). In this study, we adopt the radial basis (RBF) kernel $\exp \left(-\gamma\left\|x-x^{\prime}\right\|_{2}^{2}\right)$ for $\eta$, where $\gamma$ is the parameter for the radial basis. 


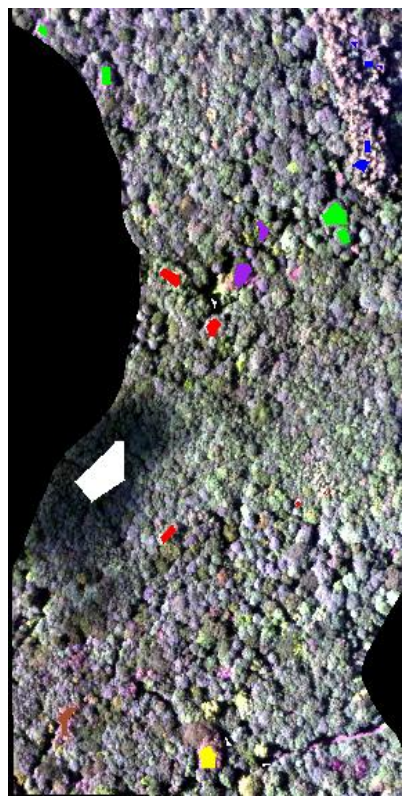

(a) Training samples

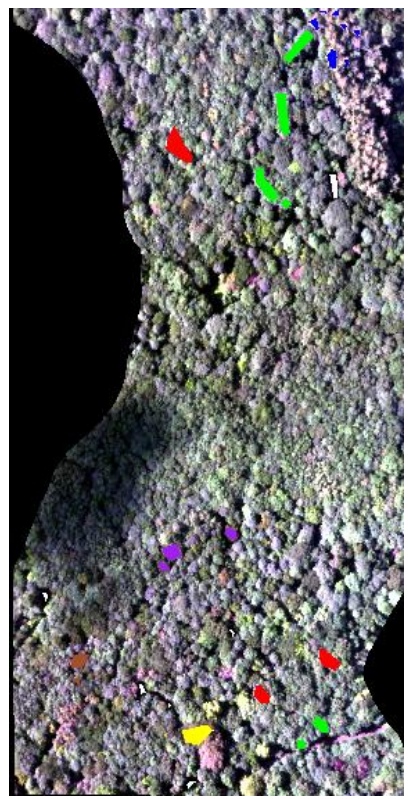

(b) Test samples

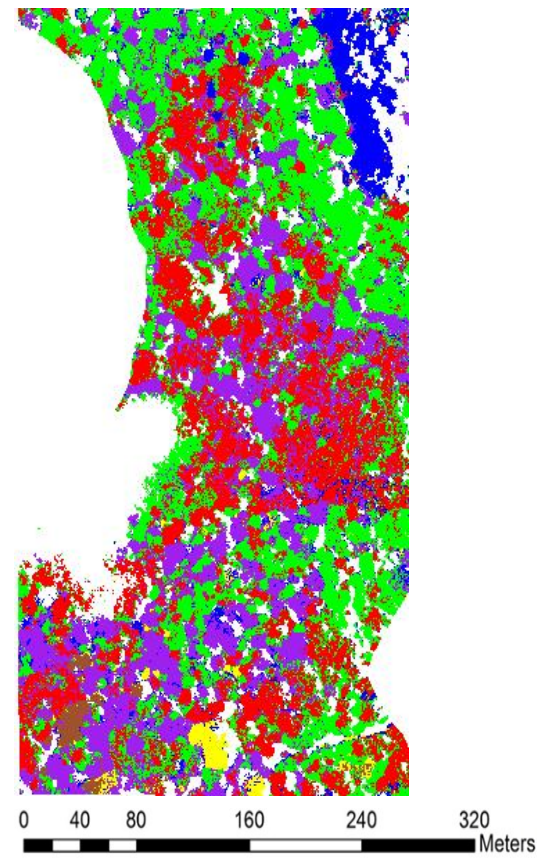

(c) Species classification map

Fig. 4.2 Training and test samples for species classification in the English dataset. The coloured polygons in (a) and (b) represent training and test samples of each species overlaid over a false colour representation of the hyperspectral imagery. The colour map (c) shows the result of the pixel-level species classification by our proposed workflow. Different colours imply different species, i.e., blue colour represents Larix decidua, green colour represents Acer pseudoplatanus, red colour represents Fraxinus excelsior, yellow colour represents Fagus sylvatica, purple colour represents Quercus robur, brown colour represents Betula spp., and white pixels indicate shaded pixels

The hyperplane $H$ is then obtained by minimising the following model

$$
\begin{array}{ll}
\min _{w, b, \xi} & \|w\|_{2}^{2}+C \sum_{i=1}^{n} \xi_{i} \\
\text { s.t. } & y_{i} \cdot\left(\left\langle w, \eta\left(x_{i}\right)\right\rangle-b\right) \geq 1-\xi_{i} \\
& \xi_{i} \geq 0
\end{array}
$$

where $C$ is a regularisation parameter and $\xi_{i}=\max \left(0,1-y_{i} \cdot\left\langle w, \eta\left(x_{i}\right)\right\rangle-b\right)$ is called a slack variable, which takes into account non-separable data. 
SVM is an intrinsically binary classifier, but it can be extended to multi-class problems by following two different strategies: one-against-one and one-against-all. In this study, we use the one-against-all rule, which solves $K$ binary problems instead of solving a $K$-class problem. We refer to [55] and references therein for an excellent introduction to SVM. A Library for SVM (libSVM) for MATLAB was used to solve the multi-class SVM problem [55]. The optimal parameters for SVM classification were manually selected by trial and error. The results obtained from manually tune parameters were evaluated using a cross validation technique, such that the best performing parameter was selected [55]. The regularisation parameter $C$ was fixed to 100 , and the parameter $\gamma$ was set to 0.5 for all experiments in Section 4.4.

Figure 4.2 (a) and (b) show the location of the training and test samples used for the pixellevel tree species classification evaluation. Table 4.2 shows the number of pixels for each species used as training and test samples. The training and test samples of the hyperspectral imagery were extracted from manually delineated ITC by means of visual inspection and field data. We considered only trees with height above $18 \mathrm{~m}$. It does make sense to filter out understory trees since the hyperspectral imagery only shows the spectral signatures of canopy trees. The ITC-level tree species classification map is obtained by extracting the pixel level map for each ITC and applying a majority voting rule $[93,78]$ to decide the species for each crown (ITC-level). Therefore, the most frequent species class inside of each ITC represents the species of ITCs.

\subsection{Results and discussion}

This section presents the experimental results of our individual tree species classification workflow. We present the results of using both PCA and rPCA in the feature extraction step of the workflow to explore whether rPCA delivers more accurate results. We refer to workflows using PCA and rPCA in the feature extraction step ITSC method and ITSC-R 

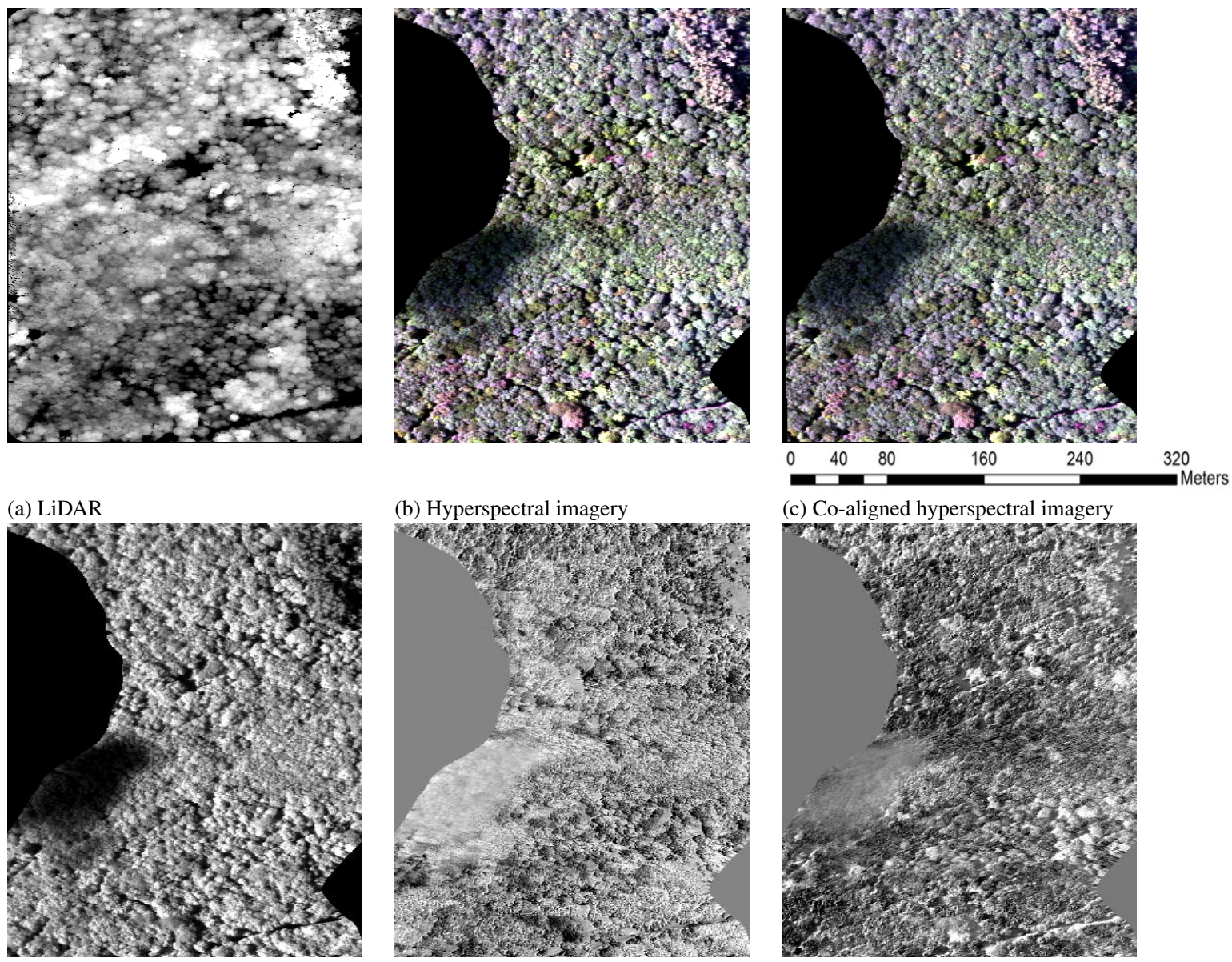

(d) First principal component

(e) Second principal component

(c) Co-aligned hyperspectral imagery

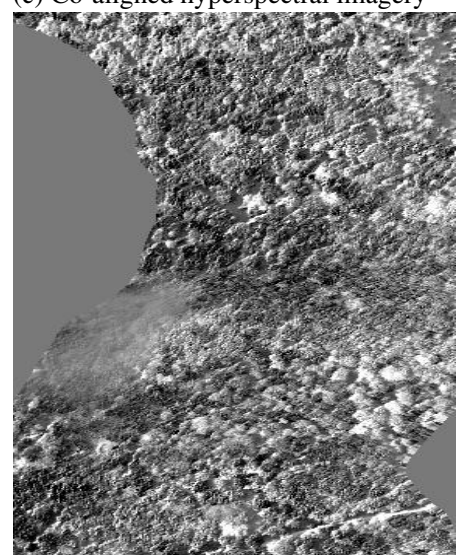

(f) Third principal component
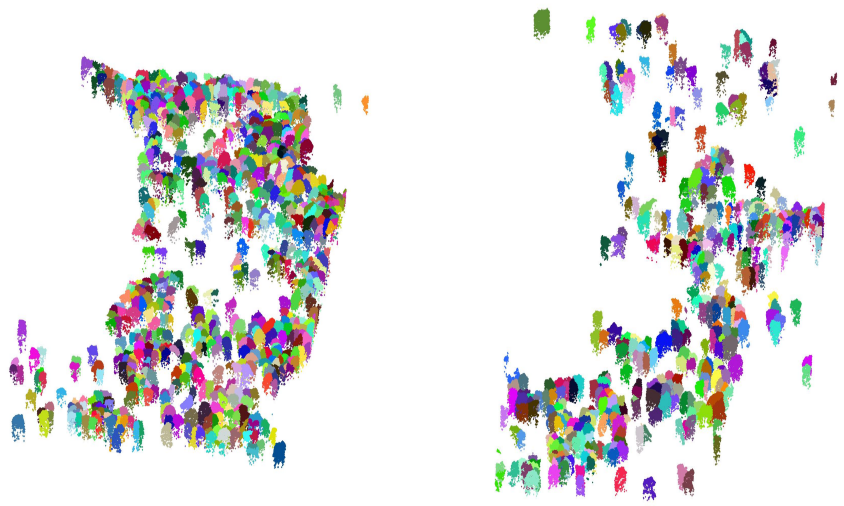

(i) ITCs of Quercus robur

(g) ITCs of Fraxinus excelsior

(h) ITCs of Acer pseudoplatanus

Fig. 4.3 The results of the images co-alignment, feature reduction and ITC delineation in our workflow in the English dataset. The first row shows the image registration between LiDAR DSM (a) and RGB true colour hyperspectral imagery (b) and the co-aligned hyperspectral imagery in RGB true colour (c). The second row shows the first three principal components (d)-(f). The third row shows examples of the MCNCP-RNC segmentation viewed obliquely (g)-(i) and different colours represent ITCs. 
method, respectively. For the results shown here, the feature extraction was applied only to the hyperspectral imagery.

Figure 4.3 shows the results of steps A, B and C of the workflow of Figure 4.1: Figure 4.3 (a) and (b) show the LiDAR (CHM) and a false colour representation of the hyperspectral imagery, and Figure 4.3 (c) shows the co-alignment result using the NGF-Curv registration method. Since the initial alignment between LiDAR and hyperspectral imagery was excellent, any errors were too small to be apparent visually in our dataset. Figure 4.3 (d) - (f) show the first three principal components obtained by the rPCA method. In our workflow, the first 20 principal components were used to delineate ITCs along with LiDAR using MCNCP-RNC, and classify species in pixel and ITC levels. Figure 4.3 (g) - (i) show the ITC delineation results using the ITC delineation method MCNCP-RNC proposed in Chapter 3.

The tree species classification was evaluated at both pixel- and ITC-level. Producer's, user's and overall accuracies were computed starting from the confusion matrix (see table 4.3, 4.4 and 4.6). The confusion matrix compares classification results with ground truth data. In particular, producer's accuracy is the ratio between the correctly classified pixels of a class and all the pixels of that class in the ground truth data. The user's accuracy is the ratio between the correctly classified pixels of a class and all the pixels classified in a given class. The overall accuracy is the ratio between the total number of the correctly classified pixels and the total number of ground truth pixels.

\subsubsection{Pixel-level tree species classification}

Figure 4.2 (c) shows the species classification results in the English data. The results over the test samples were presented in Tables 4.3 and 4.4. The overall accuracy of the ITSC-R method at pixel-level was $89.1 \%$ while that of the ITSC method was $84.8 \%$. In overall, the ITSC-R method showed better performances compared to the ITSC method. More details are given in the following. 
Regarding the individual species, for Fraxinus excelsior, the producer's accuracy was $82.3 \%$ and $87.5 \%$ for ITSC-R and ITSC methods, respectively, but the ITSC-R method performed better with respect to the user's accuracy. The ITSC-R method outperformed the ITSC method for producer's accuracy and user's accuracy of Acer pseudoplatanus. The ITSC$\mathrm{R}$ method showed better performance with respect to producer's, while user's accuracies of Quercus robur were poor for both ITSC-R and ITSC methods. The confusion matrices of both ITSC-R and ITSC methods (Tables 4.4 and 4.3) show that Fraxinus excelsior was confused mainly with Acer pseudoplatanus and Quercus robur. Acer pseudoplatanus was confused with Fraxinus excelsior and Quercus robur. Quercus robur was a major confusing factor of both Fraxinus excelsior and Acer pseudoplatanus. Indeed this is the reason of the low user's accuracy of Quercus robur. It makes sense as the ground truth data in Figure 4.4 clearly shows that these three species are dominant in the study site and often are mixed.

Larix decidua was dominant at the north east edge of the study site, and both ITSC-R method and ITSC methods successfully classified all the test samples of Larix decidua. However, Larix decidua was the major confusing factor for Fagus sylvatica pixels, so the user's accuracy of Larix decidua was poor for the ITSC method. The producer's accuracy of Fagus sylvatica was only $59.3 \%$ for the ITSC method as it was confused with Larix decidua pixels. For the ITSC-R method, the producer's and user's accuracies were $87.5 \%$ and $92.3 \%$, respectively. The producer's accuracy of Betula species were only $72.3 \%$ for ITSC method, while ITSC-R method achieved $80.0 \%$ producer's accuracy. With respect to the user's accuracy of Betula species, the ITSC-R method achieved 97.5\%, while the ITSC method only had $85.7 \%$.

Species classification at pixel level is strongly influenced by illumination effects [67, $223,122,78]$. Species classification using only sunlit pixels produce better results than considering all pixels (including shaded pixels) [67]. Thus a shadow removal step may be seen as a pre-requisite. Shadow removal can be done by manual selection $[67,108]$, ray 
Table 4.3 Species classification results from the ITSC method

\begin{tabular}{|c|c|c|c|c|c|c|c|c|c|c|}
\hline & & & & Gro & d truth & & & & & \\
\hline & Species & $\begin{array}{l}\text { Fraxinus } \\
\text { excelsior }\end{array}$ & $\begin{array}{c}\text { Acer } \\
\text { pseudoplatanus }\end{array}$ & $\begin{array}{c}\text { Larix } \\
\text { decidua }\end{array}$ & $\begin{array}{c}\text { Quercus } \\
\text { robur }\end{array}$ & $\begin{array}{c}\text { Fagus } \\
\text { sylvatica }\end{array}$ & $\begin{array}{l}\text { Betula } \\
\text { spp. }\end{array}$ & Shade & Total & $\begin{array}{c}\text { Producer's } \\
\text { accuracy }(\%)\end{array}$ \\
\hline \multirow{9}{*}{ Classification results } & Fraxinus excelsior & 401 & 35 & 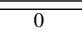 & 21 & 0 & 0 & 1 & 458 & 87.5 \\
\hline & Acer pseudoplatanus & 60 & 543 & 4 & 38 & 0 & 2 & 0 & 647 & 83.9 \\
\hline & Larix decidua & 0 & 2 & 132 & 0 & 0 & 1 & 0 & 135 & 97.7 \\
\hline & Quercus robur & 0 & 0 & 7 & 181 & 0 & 0 & 0 & 188 & 96.2 \\
\hline & Fagus sylvatica & 0 & 17 & 41 & 6 & 114 & 11 & 3 & 192 & 59.3 \\
\hline & Betula spp. & 2 & 2 & 6 & 15 & 2 & 76 & 2 & 105 & 72.3 \\
\hline & Shade & 0 & 0 & 0 & 1 & 0 & 0 & 108 & 109 & 99.0 \\
\hline & Total & 463 & 599 & 190 & 262 & 116 & 90 & 114 & 1834 & \\
\hline & User's accuracy (\%) & 86.6 & 88.2 & $\overline{269.4}$ & 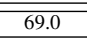 & 98.3 & 85.7 & 94.7 & & 84.8 \\
\hline
\end{tabular}

Table 4.4 Species classification results obtained by the ITSC-R method

\begin{tabular}{|c|c|c|c|c|c|c|c|c|c|c|}
\hline & & \multicolumn{7}{|c|}{ Ground truth } & \\
\hline & Species & $\begin{array}{l}\text { Fraxinus } \\
\text { excelsior }\end{array}$ & $\begin{array}{c}\text { Acer } \\
\text { pseudoplatanus }\end{array}$ & $\begin{array}{c}\text { Larix } \\
\text { decidua }\end{array}$ & $\begin{array}{l}\text { Quercus } \\
\text { robur }\end{array}$ & $\begin{array}{c}\text { Fagus } \\
\text { sylvatica }\end{array}$ & $\begin{array}{c}\text { Betula } \\
\text { spp. }\end{array}$ & Shade & Total & $\begin{array}{c}\text { Producer's } \\
\text { accuracy (\%) }\end{array}$ \\
\hline \multirow{6}{*}{ Classification results } & Fraxinus excelsior & 378 & 31 & 3 & 44 & 0 & 0 & 2 & 458 & 82.3 \\
\hline & Larix decidua & 0 & 0 & 135 & 0 & 0 & 0 & 0 & 135 & 100 \\
\hline & Quercus robur & 0 & 0 & 0 & 188 & 0 & 0 & 0 & 188 & 100 \\
\hline & Fagus sylvatica & 0 & 0 & 9 & 7 & 168 & 2 & 6 & 192 & 87.5 \\
\hline & Betula spp. & 0 & 1 & 2 & 3 & 14 & 84 & 1 & 105 & 80.0 \\
\hline & User's accuracy (\%) & 92.7 & 94.7 & 88.8 & 66.9 & 92.3 & 97.7 & 91.5 & & 89.1 \\
\hline
\end{tabular}

tracing simulation with LiDAR derived DSM [223], ITC information from LiDAR [122] or normalised difference vegetation index filtering [3]. In this study, shaded pixels were included as additional class for learning (see Figure 4.2), such that shaded pixels were detected during the pixel-level species classification process as in Dalponte et al. [74]. This strategy is particularly useful as we can detect shaded pixels without particularly processing the shaded area. The producer's and user's accuracies of shaded pixel detection were high in both ITSC and ITSC-R methods. It is well-known that the first principal component is mainly related with illumination effects [250], which may be linked with high accuracy of shaded pixel detection of our method. Although Tochon et al. [250] report that the first component of PCA is not useful for ITC delineation, it can, at least, be used for more accurate pixel-level species classification by detecting shaded pixels.

In this study, pixel-level species classification was conducted by only hyperspectral imagery because this study put more emphasis on improving species classification from hyperspectral imagery using rPCA technique. Many studies reported that using LiDAR features 
could improve species classification, since LiDAR is not influenced by illumination artefacts $[74,146,76,79]$. LiDAR intensity may provide more detailed radiometric information for guiding species classification, however, in our acquisition LiDAR intensity was controlled in non-linear way by automatic gain control, thus it was not possible to calibrate the intensity and to use it for species classification. In addition, illumination information is important for finding shaded pixels in our method, so the improvement by additional features from LiDAR may not be significant. Investigating the role of LiDAR derived metrics on species classification could provide better understanding of species classification, this is the beyond of the scope of this paper.

The size of training dataset may affect the species classification result. In this paper Approximately 1-7 crowns per each species were used to classify all species and shaded pixels, because we only selected trees visually recognisable from both LiDAR and ground data. Baldeck and Asner [27] showed that the sensitivity of species classification on sample size is dependent on the number of species and spectral separability of each species. In this study, the optimal number of tree crowns for species classification in a Savanna woodland was 10 trees for two species and 19 trees for eleven species. These numbers are larger than the number samples used in this paper. This difference might be related with the types of forest. We tested our algorithm in a temperate forest, while their study was conducted a savanna woodland. In addition, we used robust PCA to reduce 361 spectral bands to 20 principal components, therefore, direct comparison may not work. Moreover, airborne hyperspectral imagery used in this paper contained spectral signals spanning from visible to SWIR (400-2500nm) wavelength region, on the other hand, the spectrometer used in [27] had spectral coverage from the visible to NIR wavelength region (400-1000nm). The SWIR region may give more spectral separability, so it could reduce the sample size needed for species classification. Finding an optimal size of training samples in our test site requires further analysis, but we leave it for future work. 


\subsubsection{ITC-level species classification - mapping individual trees}

Species classification at ITC-level was validated using a validation software for tree segmentation $[204,89]$. This software uses tree heights and tree locations to find the best matching candidates between the ground truth and the segmented tree crowns. Specifically, 2D horizontal distance and height difference between ground truth and segmented trees are compared. During the matching process, horizontal and vertical distances within $5 \mathrm{~m}$ between ground truth and segmented trees are considered and scored. The software, therefore, is still sensitive to the initial positioning of the ground truth. To alleviate this problem, we also included two figures to evaluate species classification at ITC-level. Figure 4.4 shows the both pixel- and ITC-level species classification results along with the ground truth information. Figure 4.5 shows the map of ITCs of each species using the MCNCP-RNC method and the ground truth data. These two figures will be used to compare the patterns of species distribution across the study site.

Table 4.5 shows the accuracy of the ITC delineation results. Commission error indicates the number of false positive trees generated by our algorithm. Omission error gives the number of missing ground truth trees. Using the MCNCP-RNC method in Chapter 3, 40\% of canopy trees were correctly delineated. In a broadleaved temperate forest, it is hard to use local maxima information for ITC delineation as tree canopies are complex. As we removed trees with height below $18 \mathrm{~m}$, some canopy trees were excluded from our validation step. This also can contribute to errors. In addition, even if the validation software accounted for up to $5 \mathrm{~m}$ positioning errors of the ground truth, inaccurate positioning still caused huge errors, which will be explained in the following paragraph.

Larix decidua is dominant at the north east edge of the study site. Since Larix decidua has a conical crown, it is relatively easy to delineate its crown accurately. However, the validation results in Table 4.5 and 4.6 shows that almost $85 \%$ Larix decidua trees were omitted according to the validation results. In Figure 4.5(d) and (j), the patterns of Larix 


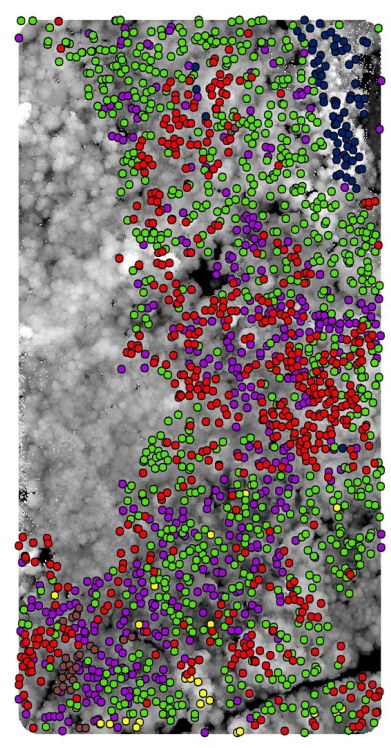

(a) ITC species mapping

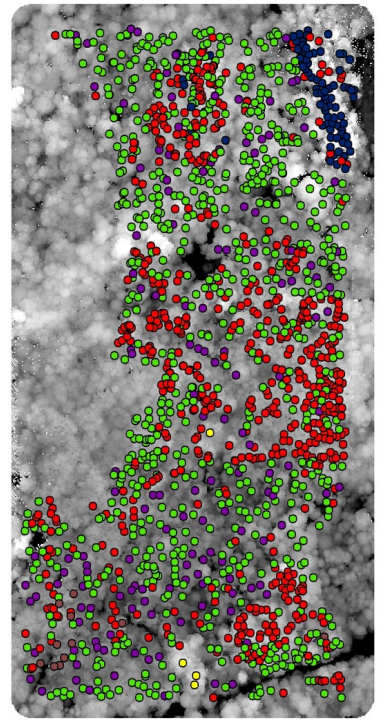

(b) Ground truth
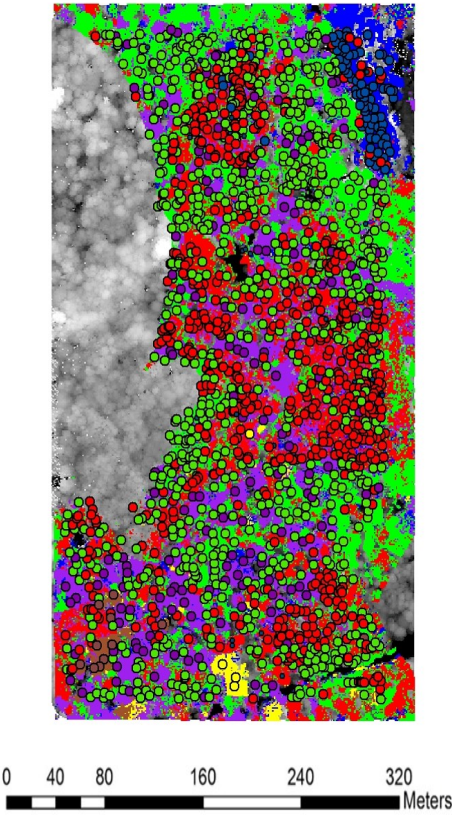

(c) Ground truth with pixel-level species classification

Fig. 4.4 Mapping individual tree species at ITC-level and ground truth over the 18ha English dataset. The background images in (a)-(c) are DSM. The coloured map in (c) is pixel-level species classification, where each colour indicates different species. The circles in different colours represents ITCs of different species. Blue colour represents Larix decidua, green colour represents Acer pseudoplatanus, red colour represents Fraxinus excelsior, yellow colour represents Fagus sylvatica, purple colour represents Quercus robur, and brown colour represents Betula spp.

decidua from segmented ITCs and ground truth field data were very similar. Therefore, the validation software did not evaluate ITC-level species classification properly. Alternatively visual analysis was also conducted comparing the number of trees and species distribution patterns over the study site.

Table 4.6 shows the confusion matrix at ITC-level, which considers only correctly assigned ITCs. The overall accuracy was only $65.8 \%$, which was lower than that at pixel level. Fraxinus excelsior classification indicates that both producer's and user's accuracies were poor, while more objective assessment of ground truth and species map in Figure 4.4 and Figure 4.5(a) and (g) show that species distribution patterns of ground truth and species 
map had a good agreement. The ITC-level classification of Acer pseudoplatanus was $72.7 \%$ and $77.1 \%$ with respect to producer's and user's accuracies. more objective comparison of Figure (b) and (h) suggested that ITC-level mapping of Acer pseudoplatanus agreed well with the ground truth data. Larix decidua was excluded from the validation as the ITC-level detection was unrealistic, but more objective assessment implies that species distribution of pixel- and ITC-level were well agreed with the ground truth data. Quercus robur had a good producer's accuracy, while its user's accuracy was only $40.1 \%$. This was in accordance with the pixel-level classification of Quercus robur. In particular, visual analysis of the ITC delineation suggests that Quercus robur was oversegmented (see Figure 4.5 (c) and (i). This might be because some branches were extracted as different local maxima, so the segmentation algorithm oversegmented the oak trees. The accuracy of Fagus sylvatica was similar with that of Quercus robur, so it had relatively high producer's accuracy and poor user's accuracy. Oversegmentation was observed in visual inspection, and indeed this was confirmed by the quantitative results: while there were only 4 trees in ground truths, twentyfive Fagus sylvatica were mapped by our workflow. This was partly because we only selected canopy trees over $18 \mathrm{~m}$, so some canopy trees were omitted. In addition, some branches of Fagus sylvatica were identified as local maxima, thereby causing commission errors. The accuracy of Betula spp. was the highest among all species in ITC-level classification. Betula spp. are located in a small area at southwest edge of the study site, so they can be mapped more easily (see Figure 4.5 (f) and (1)).

Figure 4.5 clearly shows that Quercus robur, Fagus sylvatica and Betula spp. were oversegmented. This may be related with huge commission errors of ITC delineation in Table 4.5. Since hyperspectral imagery contains illumination information, it may cause oversegmentation. Specifically, shaded pixels in hyperspectral imagery cause a bias of ITC delineation [122, 78, 250], therefore, minimising shade effects from hyperspectral imagery could alleviate oversegmentation problem. Tochon et al. [250] report that the first principal 


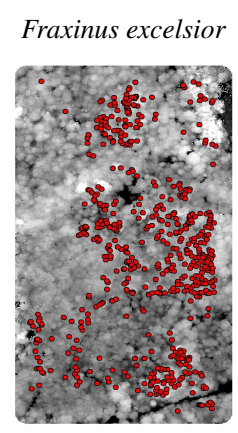

(a)

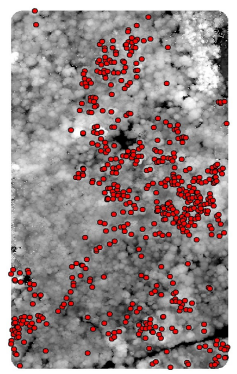

(g)

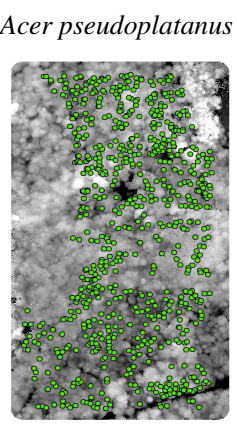

(b)

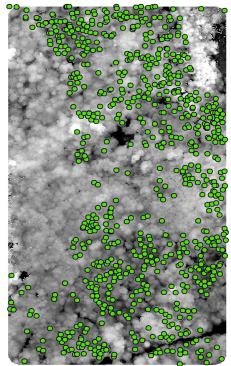

(h)

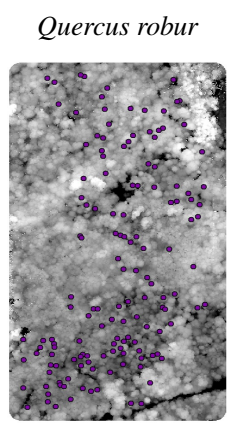

(c)

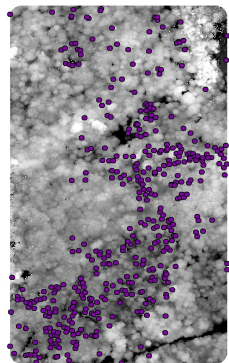

(i)

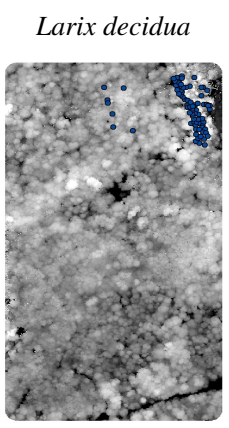

(d)

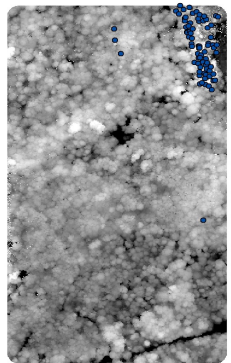

(j)

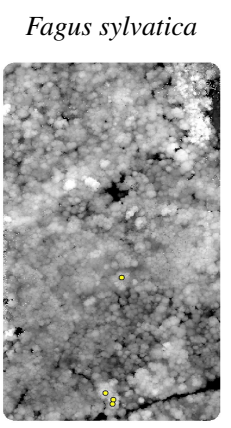

(e)

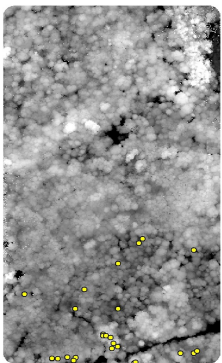

(k)
Betula spp

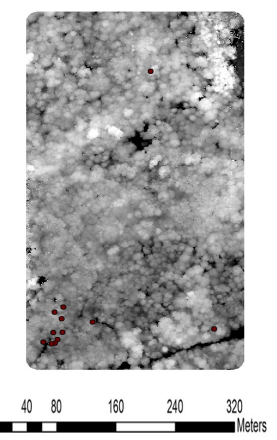

(f)

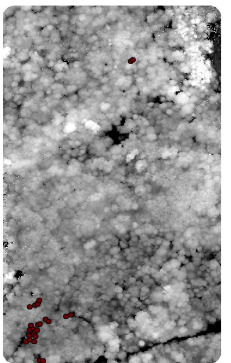

(1)

Fig. 4.5 Mapping each species at ITC-level and the ground truth. Each circle represents a single ITC. The first row shows the ground truths canopy trees over $18 \mathrm{~m}$. The second row shows the results of ITC-level species classification. Different colours imply different species, i.e., red colour represents Fraxinus excelsior, green colour represents Acer pseudoplatanus, purple colour represents Quercus robur, blue colour represents Larix decidua, yellow colour represents Fagus sylvatica, and brown colour represents Betula spp.

component of hyperspectral imagery contains most illumination information, thus removing it from ITC delineation process may help the accuracy of segmentation. In addition, MCNCPRNC algorithm assumed that the number of clusters were similar or larger than the number of priors unless priors were located too closely together in space. In other words, MCNCP-RNC has limited performance for merging clusters, while it can still find more clusters by checking further separability. Under this setting, MCNCP-RNC could cause oversegmentation.

Table 4.5 ITC delineation accuracy with commission and omission errors

\begin{tabular}{c|c|c|c}
\hline Total number & Matched & Commission error & Omission error \\
\hline \hline 1661 & 677 & 960 & 964 \\
\hline
\end{tabular}


Table 4.6 Species classification results at ITC-level.

\begin{tabular}{|c|c|c|c|c|c|c|c|c|c|c|}
\hline & & & & & d truth & & & & & \\
\hline & Species & $\begin{array}{l}\text { Fraxinus } \\
\text { excelsior }\end{array}$ & $\begin{array}{c}\text { Acer } \\
\text { pseudoplatanus }\end{array}$ & $\begin{array}{c}\text { Larix } \\
\text { decidua }\end{array}$ & $\begin{array}{l}\text { Quercus } \\
\text { robur }\end{array}$ & $\begin{array}{c}\text { Fagus } \\
\text { sylvatica }\end{array}$ & $\begin{array}{l}\text { Betula } \\
\text { spp. }\end{array}$ & $\begin{array}{c}\text { Other } \\
\text { species }\end{array}$ & Total & $\begin{array}{c}\text { Producer's } \\
\text { accuracy }(\%)\end{array}$ \\
\hline \multirow{9}{*}{ Classification results } & Fraxinus excelsior & 133 & 50 & 1 & 46 & 0 & 1 & $\overline{0}$ & 231 & $\begin{array}{l}57.8 \\
\end{array}$ \\
\hline & Acer pseudoplatanus & 53 & 243 & 2 & 35 & 1 & 0 & 0 & 334 & 72.7 \\
\hline & Larix decidua & 3 & 4 & 5 & 1 & 0 & 0 & 0 & 13 & 38.4 \\
\hline & Quercus robur & 2 & 11 & 0 & 55 & 4 & 0 & 0 & 72 & 76.3 \\
\hline & Fagus sylvatica & 0 & 1 & 0 & 0 & 3 & 0 & 0 & 4 & 75.0 \\
\hline & Betula spp. & 1 & 0 & 0 & 0 & 0 & 7 & 0 & 8 & 87.5 \\
\hline & Other species & 5 & 6 & 4 & 0 & 0 & 0 & 0 & 15 & 0 \\
\hline & Total & 197 & 315 & 12 & 137 & 8 & 8 & 0 & 677 & - \\
\hline & User's accuracy (\%) & 67.5 & 77.1 & 41.6 & 40.1 & 37.5 & 87.5 & 0 & - & 65.8 \\
\hline
\end{tabular}

\subsection{Concluding remarks}

This study investigated the possibility of species mapping using airborne remote sensing datasets. Although several algorithms have been suggested for species mapping over various types of forest, their system architectures assumed co-alignment of LiDAR and hyperspectral imagery, so it was difficult to apply directly. In addition, their methodologies for delineating ITC are mostly based on digital surface model rather than LiDAR point cloud, so both forest and ITC parameter estimation were relatively inaccurate. We introduced 3D tree delineation algorithm MCNCP-RNC in our proposed workflow, so forest analysis at species level, such as total biomass estimation of each canopy species, can be conducted more accurately. Our pixel-level species classification method showed that overall $89 \%$ of pixels were correctly assigned. However, the overall accuracy of ITC-level tree species classification was only $65.8 \%$. The low accuracy at the ITC-level classification might be related with inaccurate geopositioning of ground truth because visual analysis showed that species distribution patterns were well agreed with ground truth. Visual analysis also indicates that MCNCPRNC method oversegmented ITCs, thus causing huge commission errors. These errors might be related with illumination artefacts from hyperspectral imagery or overestimation of tree top priors taken from LiDAR data. Further research is required to evaluate our scheme with accurately georeferenced ground truth and improve ITC-level species delineation by correcting both illumination effects and overestimation of tree top priors. 


\section{Chapter 5}

\section{Physiological indices of individual trees}

\section{in Mediterranean woodland estimated}

using fused airborne LiDAR and

\section{hyperspectral imagery}

\subsection{Introduction}

Canopy species dominates carbon storage and water use, and its diversity is proportional to species distribution [17]. Under this context, canopy physiology is a key to understand spatial and temporal variability of carbon, nutrient and water cycles [17]. Specifically, plant species synthesise a number of compounds for various purposes. Those compounds can be classified into three broad functional types: light capture and growth, longevity and defence, and maintenance and metabolism, which are closely linked to energy flux, nutrient cycling and species diversity of forests [20]. Canopy physiological measurements can provide a tool for monitoring species responses to environmental stress [227, 226] and chemical diversity in 
Physiological indices of individual trees in Mediterranean woodland estimated using fused airborne LiDAR and hyperspectral imagery

various taxonomy $[17,18]$. However, traditional ecological sampling methods are laborious and only cover small areas, so it is hard to map species and monitor canopy physiology efficiently at large spatial and temporal scales.

Recent advances of airborne multi-sensor imaging can greatly expand the scale of ecological studies as they can be used to estimate canopy physiological responses and detect tree species $[239,66]$. Airborne hyperspectral imaging spectroscopy measures surface reflectance in narrow spectral bands with spatial resolution of less than $3 \mathrm{~m}$. LiDAR provides 3D point cloud informing vegetation structures. The combination of LiDAR and hyperspectral imagery enables us to identify the physical and chemical properties of leaves within single crowns [17]. The spectral signatures of canopy leaves are related to canopy-leaf chemical and physical properties and therefore it is possible to map species distribution if the chemical constituents and spectral properties of canopy leaves of each species are known [17]. A number of studies on canopy-leaf chemical and spectral diversity have been conducted and successfully identified canopy species in tropical forests [16, 20, 19, 21, 23, 22]. Although those studies considered 21 foliar traits to identify the spectral and chemical diversity of tropical forests it has been suggested that they could be reduced to 14 considering orthogonal property of chemicals $[19,21]$

Although hyperspectral data enable to analyse species at an ITC scale, intra- and interspecific physiological responses of each species remain unclear [124]. Asner et al. [20] argue that the physiological responses along environmental gradients in tropical rainforests are mainly driven by taxonomical changes rather than intraspecific variation. However, Hesketh and Sánchez-Azofeifa [124] showed that spectral signatures in tropical forests could vary with seasonal variation such that intraspecific variation may be large enough to influence spectral analysis. Our research pioneers a technique to monitor physiological indices at ITC level in a Mediterranean woodland, where two Quercus species (Q. suber and $Q$. canariensis) are dominant. The Mediterranean ecosystems we studied contain strong seasonal variation of 
temperature and precipitation. We identified whether hyperspectral and LiDAR duality could capture intra- and interspecies variation in physiological indices. We also analysed the extent of physiological indices variation along environmental gradients.

Our initial approach in Chapter 4 attempted to analyse hyperspectral images at an ITC level in reference to field data. This chapter provides a modification of the generic workflow in Chapter 4 for analysing canopy physiology. The main objectives of this study are to explore the relationship between intraspecific variation of physiological indices and environmental gradients; to examine the reliability of physiological indices developed for hyperspectral analysis to determine to what extent taxonomic changes influence physiological traits in Mediterranean species. Since we do not have field measured data, it is hard to confirm our finding in standard ways. Alternatively, we compared our results with the previous studies on Mediterranean ecosystem $[252,107]$ and plant physiological responses with environmental gradients [81, 227, 226, 68, 260, 222].

\subsection{Data description and design of experiments}

\subsubsection{Study area}

Los Alcornocales natural park is located in Andalusia, southern Spain $\left(36^{\circ} 11^{\prime} \mathrm{N}, 5^{\circ} 33^{\prime} \mathrm{W}\right)$. Figure 5.1 shows the study sites in Los Alcornocales that include the most extensive $Q$. suber forest in the Mediterranean region $[233,4]$ with the other common species being $Q$. canariensis [233]. Annual precipitation ranges from $1210 \mathrm{~mm}$ in the mountainous regions to $665 \mathrm{~mm}$ in the lowlands. The mean air temperature is $16-18 \mathrm{C}^{\circ}$ [147]. The elevation of the study site ranges from 100 to $800 \mathrm{~m}$. 


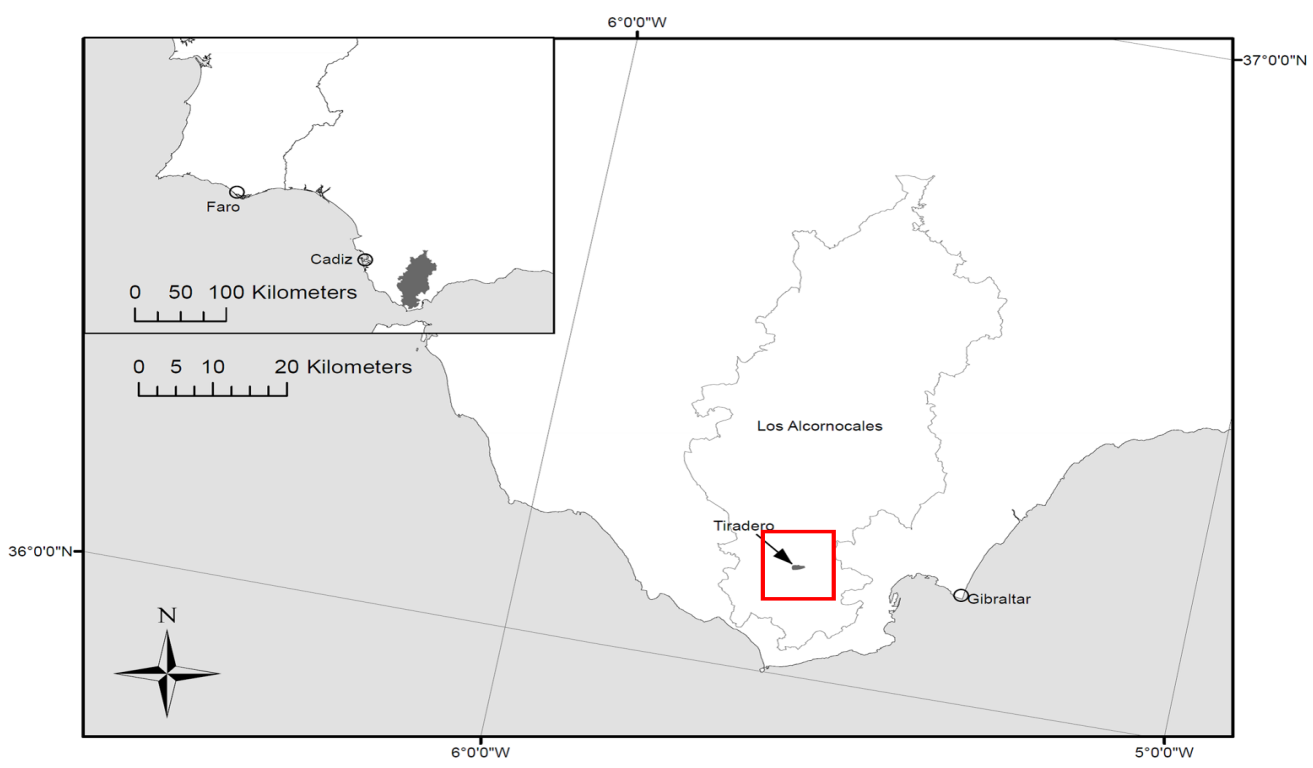

Fig. 5.1 Location of Los Alcornocales, Southern Spain (provided by Dr. Simonson). The red rectangular box indicate the places where hyperspectral and LiDAR data were obtained.

\subsubsection{Airborne image collection and pre-processing}

Airborne surveying was conducted in Tiradero region of the Los Alcornocales Natural Park on 10 April 2011 (see Figure 5.1). The airplane flew at a nominal height above ground of approximate $3000 \mathrm{~m}$. Hyperspectral data were gathered using the AISA Eagle covering 400-970 nm wavelength. The hyperspectral datasets were calibrated by Specim Ltd. (Finland) and the Airborne Research and Survey Facility of the UK's Natural Environment Research council (NERC-ARSF) such that the datasets recorded in digital numbers (DN) were converted to spectral radiance before being provided to us. The units of spectral radiance are $\mu \mathrm{W} \mathrm{cm}{ }^{-2} \mathrm{sr}^{-1} \mathrm{~nm}^{-1}$ where $\mathrm{sr}$ - steradian - is the SI unit of solid angle. The quality of the data was checked and controlled by ARSF twice before delivery. However, considerable noise appeared close to the edge of the sensor's coverage of the electromagnetic spectrum, thus spectral information from the Eagle sensor below $400 \mathrm{~nm}$ and above $970 \mathrm{~nm}$ was excluded. 
In order to investigate physiological properties of ITCs, the spectral data needs to be converted from radiance to reflectance. Ideally, this process should use "ground truth" surface reflectance data (i.e. squares of white cloth laid on the ground). We could not use this empirical method for obtaining reflectance as we did not have such data. Instead, we had to estimate reflectance using radiative transfer models. The atmosphere contains water vapour, carbon dioxide and other atmospheric tracer gases, which absorb and scatter radiation [Richter and Schläpfer].This is why we applied atmospheric correction using ATCOR-4 (Rese, Switzerland) [Richter and Schläpfer].

Reflectance properties vary with view angle and sun position known as Bi-direction reflectance distributional function (BRDF) [Richter and Schläpfer]. Hyperspectral imagery is sensitive to BRDF effects when several flight lines have to be stacked together as each flight line incorporates different view and solar geometry. BRDF effects on airborne hyperspectral vegetation analysis have been known to jeopardise the validity of species prediction mapping of Savanna woodland [71] due to anisotropic scattering resulting in strong artefacts. BRDF effects presented in airborne hyperspectral imagery can be significantly alleviated by the Li-Ross model [230, 259]. We adopted the ATCOR-4 built-in function, which is based on the Li-Ross model, to offset BRDF effects of 17 different flight lines. However, BRDF effects remained in four flight lines, which were not included in the further analysis.

Hyperspectral data were georeferenced by APL software provided by the NERC Airborne Research and Survey Facility (NERC-ARSF). Since hyperspectral images were gathered from 17 different flight lines, constructing a multiple flight-line mosaic was required using a layer stacking algorithm in ENVI 5.0 (Exllis, United States). Geographical mismatches of about 3-9m were observed in overlapped region between flight lines. This may be because flight navigation processing for the hyperspectral sensor was inaccurate thus geometrical correction of hyperspectral imagery was conducted using a coarse resolution digital elevation model, which caused topographic distortion. 
Physiological indices of individual trees in Mediterranean woodland estimated using fused airborne LiDAR and hyperspectral imagery

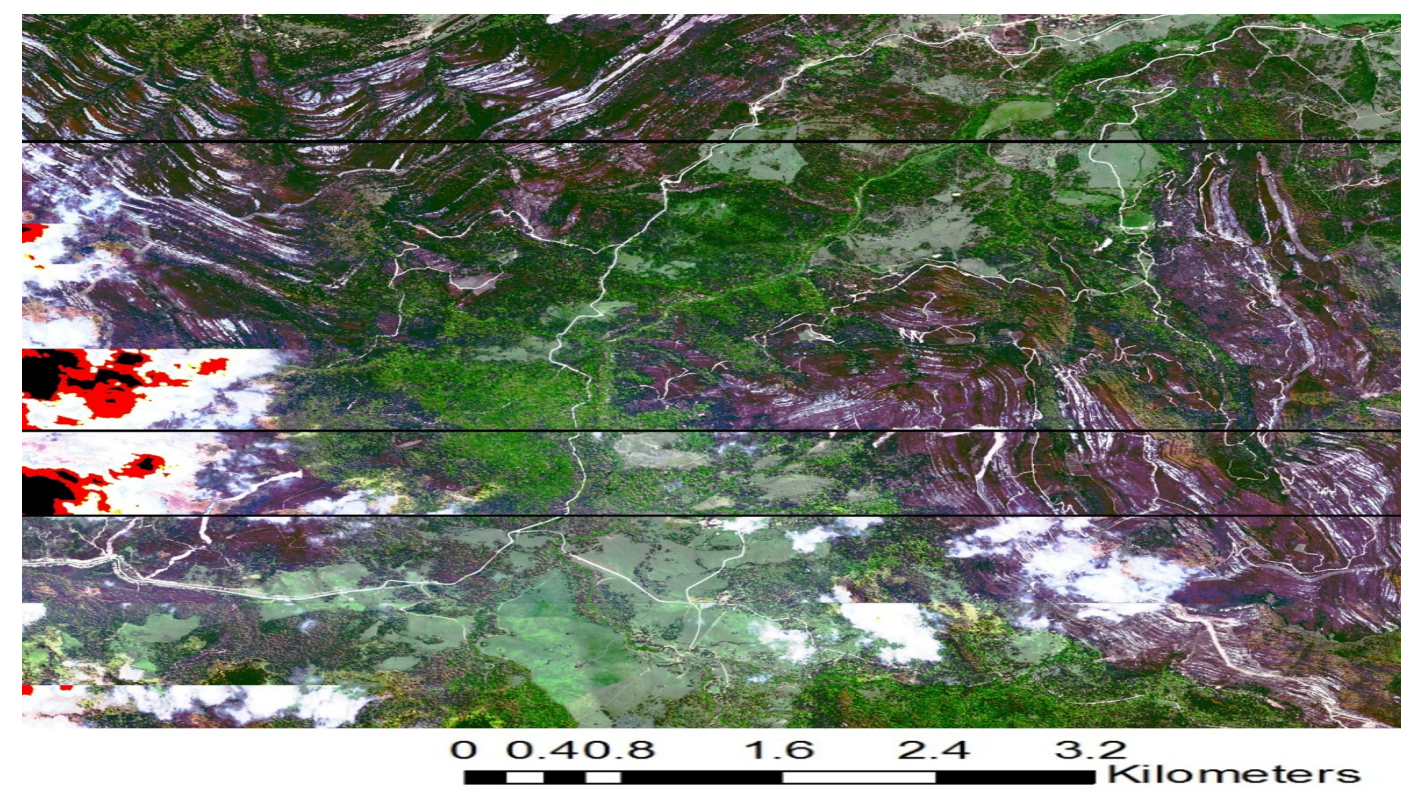

Fig. 5.2 The RGB true colour composite (460, 550 and 640nm) of hyperspectral imagery

Figure 5.2 shows the RGB true colour composite of hyperspectral imagery of the study site. Green colour represents green vegetation. Purple colour indicates ground soils. White shows clouds, roads and bedrocks. There are horizontal black lines across the hyperspectral imagery because of the excluded flight lines.

The LiDAR dataset was collected simultaneously with hyperspectral imagery by Leica ALS-50 II sensor. The field of view was set to $12^{\circ}$ in order to minimise occlusions at the edge of scan angles. The LiDAR point density was 2 point per metre squared. Each LiDAR point contains not only geometry but also associated intensity, which correlates with the proportion of a pulse's energy. However, as LiDAR intensity values are manipulated by automatic gain control system during the acquisition process, radiometric calibration of LiDAR intensity is not available. NERC-ARSF pre-processed the LiDAR data and georeferenced it to the Universal Transverse Mercator (UTM) projection with WGS-84 datum. Then, the LiDAR dataset was pre-processed using the Toolbox for LiDAR Data Filtering and Forest Studies (TIFFS) package. As LiDAR raw point clouds contained both 
h

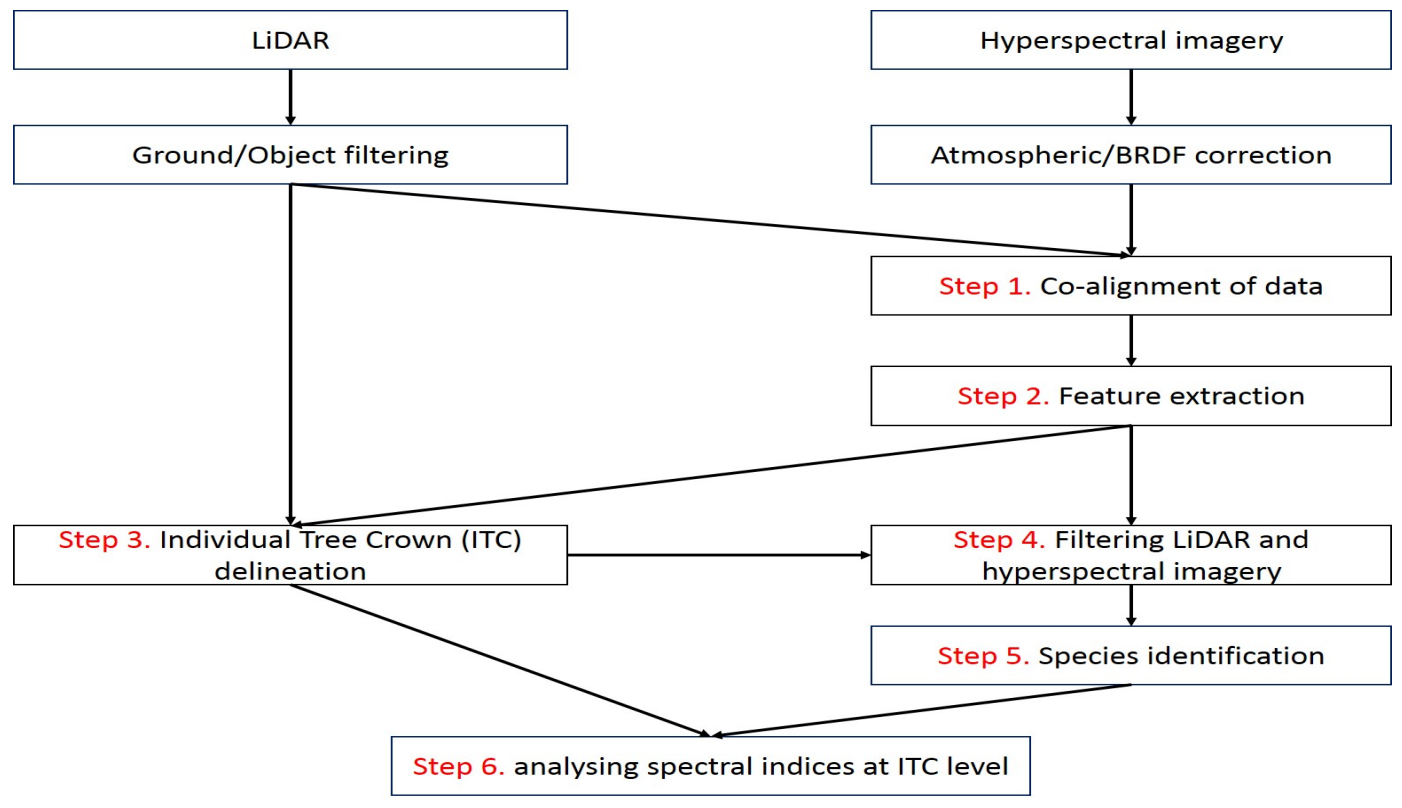

Fig. 5.3 The workflow for analysing spectral indices at ITC level

ground and vegetation structures, vegetation point clouds were extracted and normalised with ground elevation by TIFFS to create a CHM.

\subsubsection{Multi-sensor data processing and analysis}

Figure 5.3 shows the workflow for analysing spectral indices at ITC level. This is a modification of the species classification workflow in Chapter 4. Inaccurate georeference resulted in misalignment of LiDAR and hyperspectral imagery thus co-registration was required (Step 1). The high dimensionality of hyperspectral imagery could lead to inaccurate ITC delineation and species classification, which can be avoided by a dimensional reduction technique (Step 2). ITCs are delineated using LiDAR and hyperspectral imagery (Step 3). As shown in Figure 5.2 hyperspectral data contained not only vegetation (green colours) but also bedrocks, roads and other objects (purple, brown and white colours). We masked non-vegetation objects out using LiDAR and a spectral index calculated using the hyperspectral imagery. Since 
Physiological indices of individual trees in Mediterranean woodland estimated using fused airborne LiDAR and hyperspectral imagery

hyperspectral imagery is sensitive to optical properties of vegetation, filtering out unnecessary information is an important step to obtain reliable results (Step 4). Two oak species are identified using hyperspectral imagery (Step 5). Finally, spectral indices were computed and analysed at ITC level (Step 6).

Our proposed ITC-level spectral analysis consists of multiple steps, each dedicated to a particular process in the overall workflow 5.3. We elaborate on those steps below.

\section{Step 1: Co-alignment of data}

Although hyperspectral imagery and LiDAR were collected simultaneously, geographical mismatches were presented between different flight lines and sensors. These errors should be corrected explicitly as misalignment in high resolution airborne imagery leads to inaccurate results. This study adopted NGF-Curv described in Chapter 2 to co-align hyperspectral and LiDAR images.

\section{Step 2: Feature extraction}

As shown in Chapter 3 and 4, extracting feature information from the hyperspectral imagery is a key step to delineate ITC and classify species. The robust PCA described in Chapters 3 and 4 was used to reduce dimensionality of hyperspectral imagery. In this study, 20 principal components were extracted and used for ITC delineation and species classification.

\section{Step 3: ITC delineation}

As LiDAR and hyperspectral imagery were co-aligned, we could delineate ITCs using both datasets. ITC delineation was performed using the MCNCP-RNC method introduced in chapter 3. The TIFFS software (Globalidar, United States) was used to extract local maxima from the interpolated LiDAR surface [61]. These local maxima were used as priors 


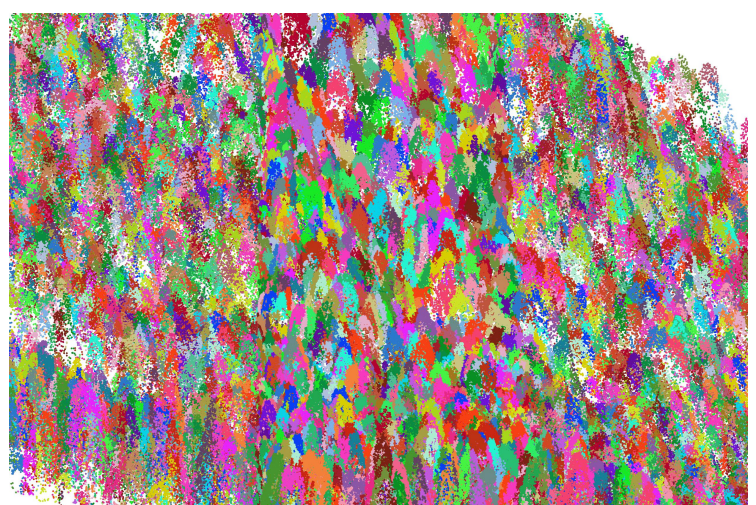

(a)

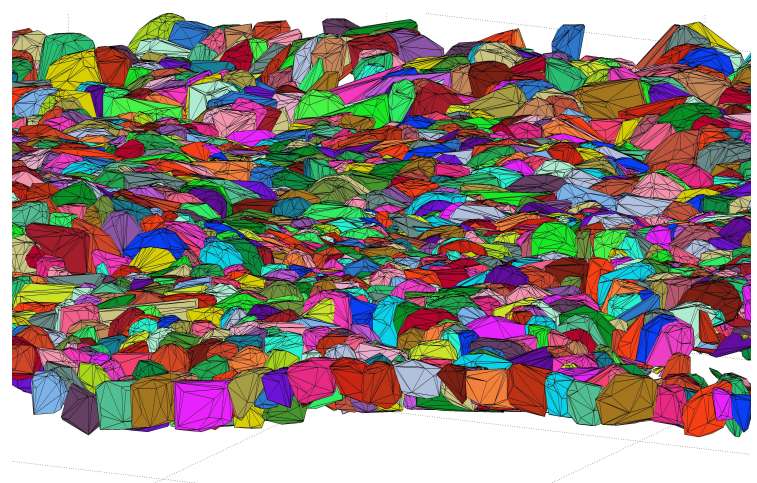

(b)

Fig. 5.4 Results of tree delineation: (a) scatter plots of ITCs, and (b) 3D shapes of ITCs generated by convex hull.

for the MCNCP-RNC algorithm. Figure 5.4 shows the results of ITC delineation using MCNCP-RNC in point clouds (a) and convex hull (b).

\section{Step 4: Filtering hyperspectral imagery}

Hyperspectral imagery needs to be filtered before computing spectral indices as they are sensitive to signals coming from objects not part of the vegetation. The first filtering step involved removing all pixels that were not occupied by trees: as individual tree crowns were distinguished by Step 5.2.3, we discarded all pixels outside tree crowns. Since small trees could have different spectral qualities to tall ones, we only included individuals higher than five meters and with canopy volume over $500 \mathrm{~m}^{3}$.

The second filtering algorithm involved calculating the Normalised Difference Vegetation Index (NDVI) and using this as a filter to remove areas of bedrock, cloud and other objects that still remained due to registration errors. NDVI is designed to identify vegetated areas from satellite or hyperspectral images [231]. Reflectance of red $(680 \mathrm{~nm})$ and near infra-red $(800 \mathrm{~nm})$ was used for calculating NDVI in Table 5.1. By selecting pixels for which NDVI $>0.8$, we effectively removed the remaining non-vegetated areas. In addition, NDVI is a good indicator of Leaf Area Index (LAI) until it saturates. LAI is one of the most important 
Physiological indices of individual trees in Mediterranean woodland estimated using fused airborne LiDAR and hyperspectral imagery

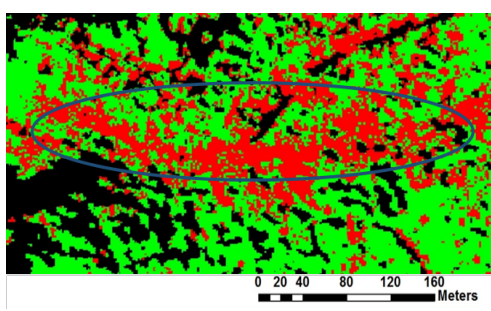

(a)

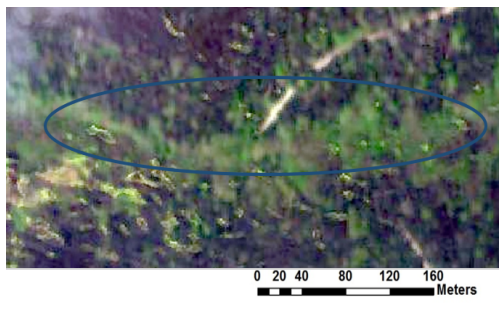

(b)

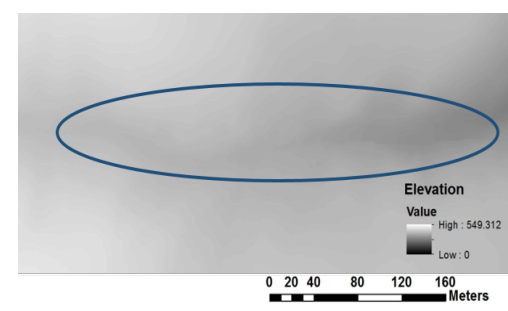

(c)

Fig. 5.5 Examples of two oak species distribution at valley bottom. Each panel shows the results of species classification (a), RGB composites of hyperspectral imagery (b) and elevation map (c), respectively. $Q$. canariensis and $Q$. suber are shown in red and green colours in (a). The dark colour in (a) represents filtered pixels, which are either ill-conditioned or non-vegetation. The blue circle indicates the location of valley bottom.

factors for analysing canopy spectra, so the high NDVI filter ensures high LAI values of the canopy spectra, minimising LAI effects on spectral indices [11]. The two filtering processes successfully managed to filter out all areas other than woodlands.

The third filtering algorithm involved removing shaded pixels from hyperspectral imagery. As hyperspectral imagery does not contain any geometrical information, LiDAR data was used to compute illumination over the surface instead. The hillshade function in ArcGIS version 10.2.2 (ESRI, United States) was used to compute an illumination map [223]. The hillshade function created shaded relief imagery using a geometry map, azimuth and zenith of light source (sun position in our case). The light source was considered to be at infinity. Pixels over $65 \%$ light illumination were selected and used for further analysis.

\section{Step 5: Species identification}

Identifying species from LiDAR and hyperspectral imagery is a key step to understand inter- and intraspecific variation of spectral indices. We adopted a species classification technique similar to that described in Chapter 4. The study area was dominated by only two oak species (Q.canariensis and $Q$ suber), and these species could be identified visually from hyperspectral imagery. As shown in Figure 5.5, Q.canariensis were dominant at valley 


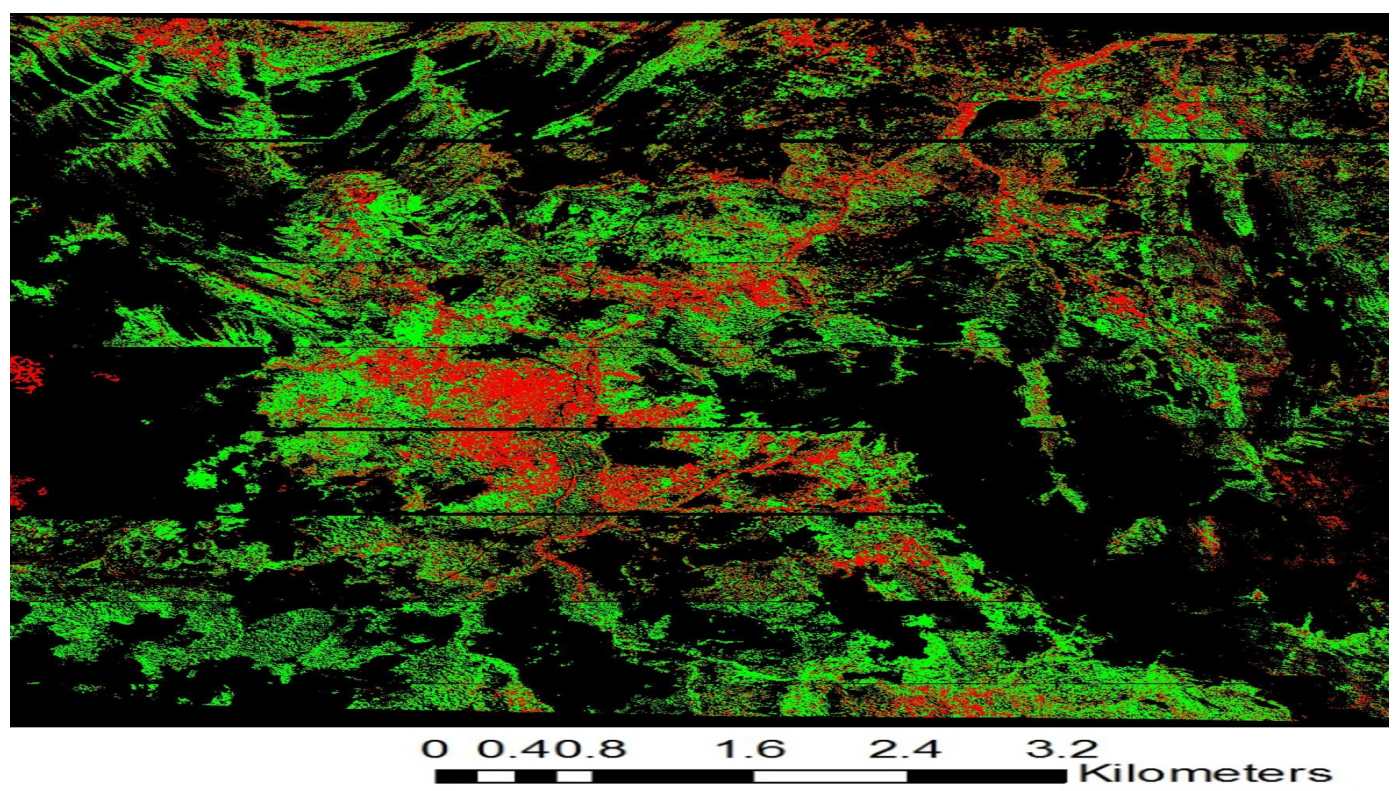

Fig. 5.6 Species classification results of hyperspectral data. Q. canariensis and $Q$. suber are shown in red and green colours, respectively.

bottom and had bright green leaves, while Q.suber were located at drier slope and had dark green leaves [252]. Spectral signatures of two oak species were trained by manually selected patches because ground truth was not available in this test site. Twenty principal components of the hyperspectral imagery extracted in Step 2 were used to train a support vector machine (SVM) algorithm to recognise the two oak species. Figure 5.6 shows the classification result of $Q$. suber in green and $Q$. canariensis in red over $35 \mathrm{~km}^{2}$ Spanish woodland. Since ground truth was not available in this test site, classification accuracy was not measured, but only visually inspected.

\section{Step 6: analysing spectral indices at ITC level.}

Tree top pixels of 59962 Q. suber and 31784 Q. canariensis samples were obtained and used to calculate chemical indices. Tree top pixels were extracted at the highest point of each ITC. This final step helped to obtain representative spectra from individual crowns such that intra- and interspecific variation of spectral indices of ITCs could be analysed. The 
Physiological indices of individual trees in Mediterranean woodland estimated using fused airborne LiDAR and hyperspectral imagery

Table 5.1 Spectral indices for estimating biophysical and chemical properties of vegetation. $\mathrm{R}$ is reflectance and subscripts give the wavelengths in $\mathrm{nm}$

\begin{tabular}{l||c|c}
\hline \multicolumn{1}{c||}{ Spectral Indices } & Equation & References \\
\hline \hline Normalised Difference Vegetation Index & $\frac{R_{800}-R_{680}}{R_{800}+R_{680}}$ & Rouse et al. [231] \\
\hline Photochemical Reflectance Index & $\frac{R_{531}-R_{570}}{R_{531}+R_{570}}$ & Gamon et al. [102] \\
\hline Red/Green Ratio Index & $\frac{\sum_{i=600}^{699} R_{j}}{\sum_{i=500}^{599} R_{i}}$ & Gamon and Surfus [104] \\
\hline Carotenoid Reflectance Index & $\frac{1}{R_{550}}-\frac{1}{R_{700}}$ & Gitelson et al. [112] \\
\hline Anthocyanin Reflectance Index & $\frac{1}{R_{510}}-\frac{1}{R_{550}}$ & Gitelson et al. [111] \\
\hline Water Band Index & $\frac{R_{900}}{R_{970}}$ & Peñuelas et al. [215] \\
\hline
\end{tabular}

digital elevation model obtained from LiDAR data was used to find the relationships between chemical indices and environmental variables. In addition, aspect and slope were calculated using the spatial analyst toolbox in ArcGIS version 10.2.2. Variation of chemical indices with respect to environmental variables was analysed using least-squares regression, comparing the fit of linear and quadratic functions, and testing for statistical significance using F-tests.

Inter- and intraspecific physiological traits over the research area were examined using five different indices: Photochemical Reflectance (PRI), Red/Green Ratio (RGRI), Carotenoid Reflectance (CRI), Anthocyanin Reflectance (CRI) and Water Band (WBI). Table 5.1 gives their equations, and we explain their physiological meaning below:

PRI was designed to estimate the ratio between two different epoxidation states of xanthophyll pigments, which is related to photochemical efficiency [102]. Garbulsky et al. [105] examined the scientific literature to identify the relationship between PRI and other 
eco-physiological variables, finding that PRI is a good indicator of photosynthetic light use efficiency (LUE), which is a ratio between carbon uptake and light absorbed by vegetation. Asner et al. [11] used PRI to examine the impact of environmental variables on plant physiology using Hyperion satellite imagery. The index was calculated from the normalised difference of the wavebands, at $531 \mathrm{~nm}$, which is sensitive to the changes in xanthophyll pigments versus reference waveband at 570nm (see Table 5.1) [103, 29, 239, 105] .

RGRI was designed to predict anthocyanin contents [104]. As shown in Table 5.1, it measures the reflectance ratio between the sum of Red bands $(600-699 \mathrm{~nm})$ and the sum of Green bands (500-599nm), thus comparing relative contents of anthocyanins to that of chlorophyll. It has been used to estimate LUE, canopy stress and forest carbon assessment along with PRI $[11,125]$.

CRI was designed to estimate carotenoids contents. Carotenoids consist of two carotenes ( $\alpha$ and $\beta$ ) and five xanthophylls (lutein, zeaxanthin, violaxanthin, antheraxanthin and neoxanthin) [112]. Retrieving carotenoids from foliar spectra is challenging because absorption and reflectance curves of carotenoid content overlaps those of chlorophyll [112]. In order to identify relative carotenoid content, chlorophyll contribution from the reflectance at $510 \mathrm{~nm}$, needs to be removed. Reflectance at 550nm was used as a reference [112].

ARI was also desgined to predict anthocyanin contents. It is calculated by removing the contribution made by chlorophyll to absorption at 550nm and observing the residual absorption. The absorption curve of anthocyanin overlaps with that of other foliar pigments. Gitelson et al. [111] showed the effect of anthocyanin by comparing reflectance and absorption spectra of leaves which contained equal chlorophyll contents but different anthocyanin contents, finding that absorption and reflectance at 550nm varied while absorption and reflectance at 700nm remained stable as anthocyanin content changed[111].

WBI was used to estimate plant water content [215]. It measured the ratio between a water absorption band at $970 \mathrm{~nm}$ and a reference band at $900 \mathrm{~nm}$. It has been previously 
Physiological indices of individual trees in Mediterranean woodland estimated using fused airborne LiDAR and hyperspectral imagery

used to estimate canopy water content of Mediterranean species from ground reflectance measurement and airborne hyperspectral imagery [216, 229].

\subsection{Result}

Figure 5.7 and 5.8 show the regression analysis between five spectral indices and elevation gradients for $Q$. suber and $Q$. canariensis, respectively. The colours of scatter plots in Figure 5.7 and 5.8 show the density of observations scaled from blue to red, i.e. red indicates high frequency of observations, and blue means low density of observations fitted using the the dscatter function in MATLAB. Table 5.2 shows the regression coefficients for the regression models.

PRI variation of both species was modelled by quadratic linear regression. As shown in Figure 5.7a and 5.8a, Photochemical Reflectance Index (PRI) in both species tended to decrease with altitude and R-squared values were approximately 0.2 for both species (Table 5.1. The regression models for both species clearly showed that the index decreased with altitude while aspect and slope had little influences on the variation of PRI. The regression fitting was marginally improved if elevation, aspect and slope were all considered, but the primary influence was altitude.

RGRI increased for both $Q$. suber and $Q$. canariensis as elevation increased. Quadratic regression models were used RGRI variations of both species with respect to elevation changes. As shown in Table 5.2, the R-squared values of the regression models between RGRI and elevation gradients were 0.16 for $Q$. suber and 0.21 Q. canariensis, so RGRI could explain environmental gradients of $Q$. canariensis better than those of $Q$. suber. Although the models including aspect and slope showed marginal improvement, main variance could be explained solely by elevation gradients.

Carotenoid contents, inferred from CRI, was almost independent of elevation (see Figure 5.7b and 5.8b). Regression models between CRI and elevation changes were too weak to 


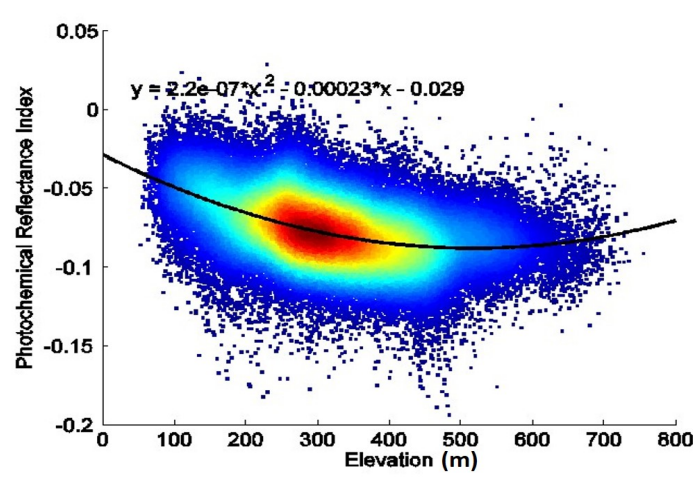

(a)

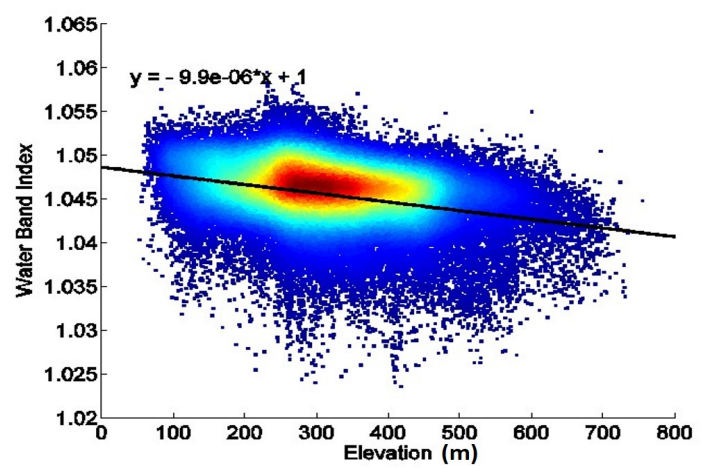

(c)

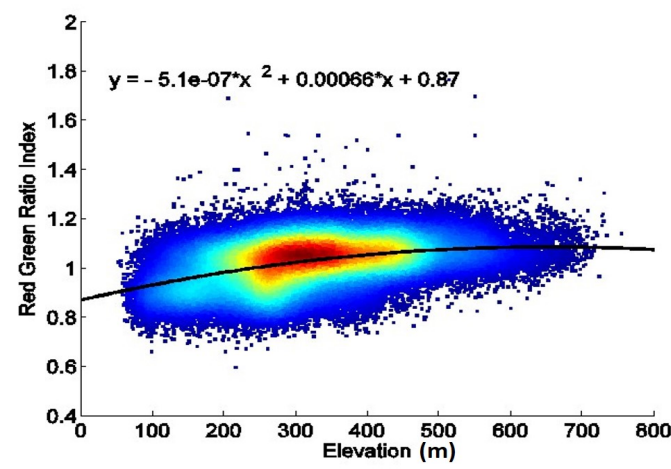

(e)

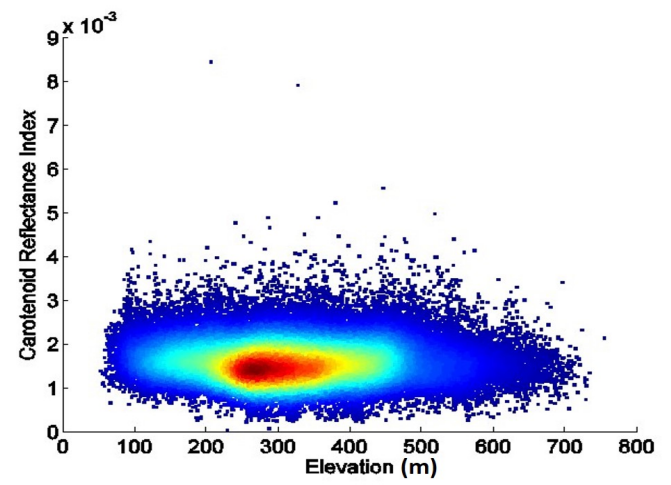

(b)

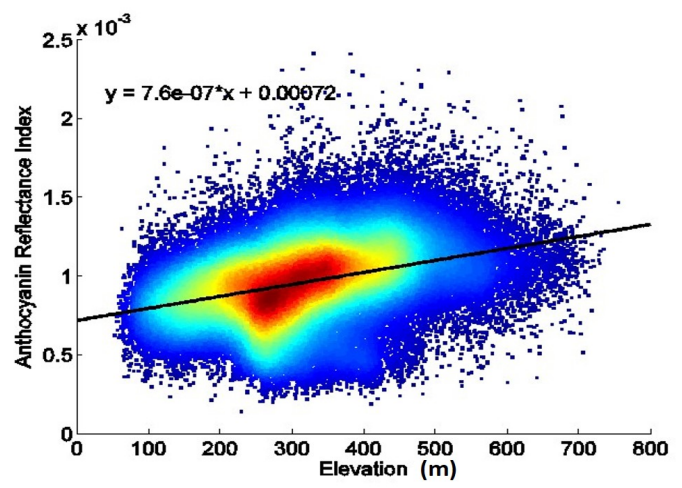

(d)

Fig. 5.7 Variation in (a) PRI, (b) CRI, (c) WBI (d) ARI (e) RGRI obtained from the hyperspectral imagery of 59962 Q. suber trees with respect to altitude. Blue line indicates fitting curve. 


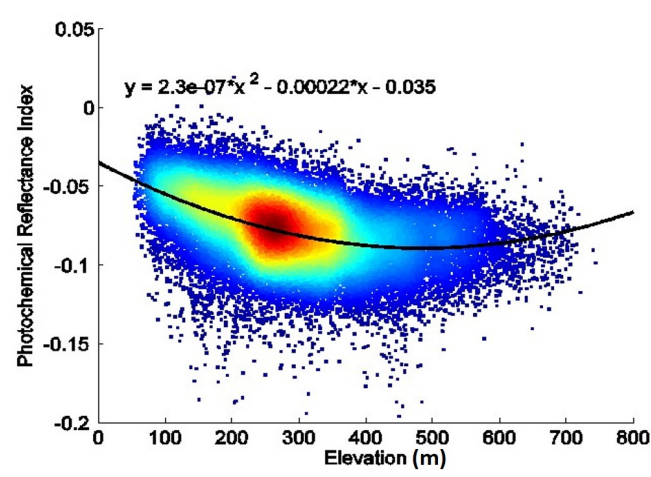

(a)

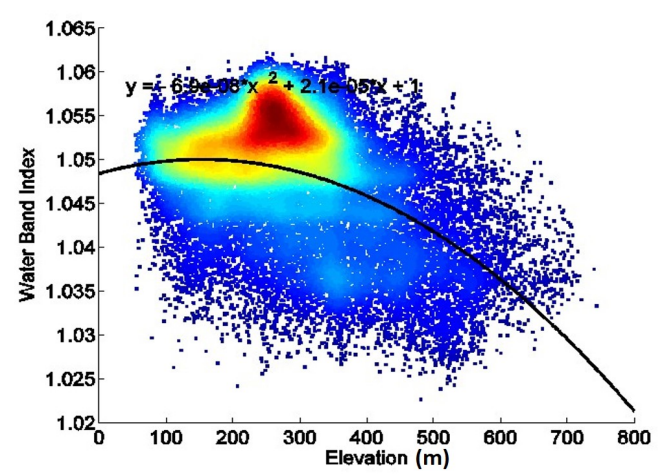

(c)

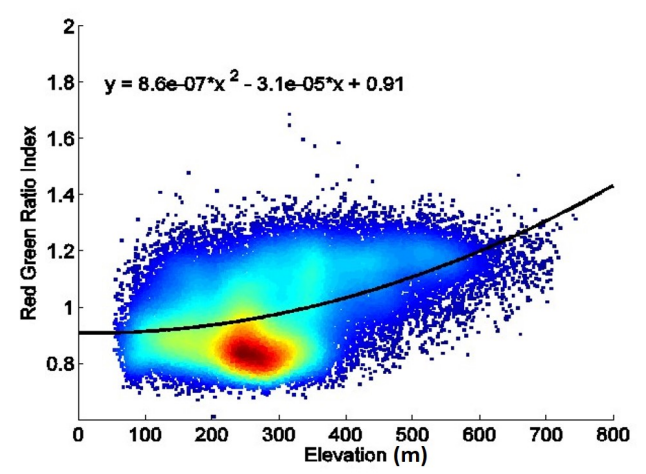

(e)

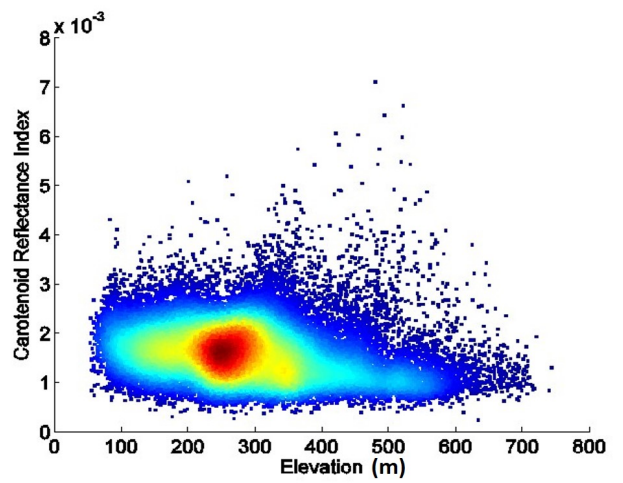

(b)

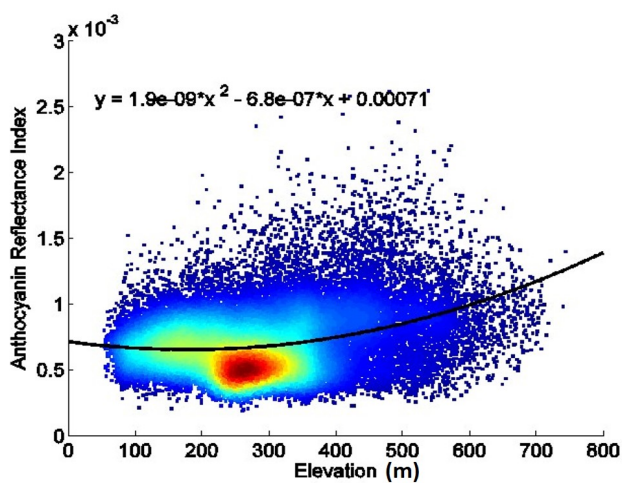

(d)

Fig. 5.8 Variation in (a) PRI, (b) CRI, (c) WBI (d) ARI (e) RGRI obtained from the hyperspectral imagery of $31784 Q$. canariensis trees with respect to altitude. Black line indicates fitting curve. 
extract a meaningful relationship $\left(R^{2} \leq 0.05\right)$. The relationship between carotenoids and other environmental variables (aspect and slope angle) was also investigated, but neither variable influence CRI significantly. The regression model including all environmental variables had slightly better R-squared value $(\leq 0.09)$ than the model solely based on elevation in Q. canariensis. However, the R-squared value was still too low to suggest any meaningful relationships between carotenoids and environmental gradients.

The relationships between ARI variations and elevations for both species were higher than those of CRI variations. Approximately $10 \%$ of ARI variations were explained by altitude changes in both species (see Table 5.2). These values were still too low to be used for any meaningful indicator. The slope and aspect changes were independent with ARI variations. In other words, ARI had very weak or no relationship with respect to environmental gradients.

WBI variation were shown in Figure 5.7c and 5.8c. WBI of both $Q$. suber and $Q$. canariensis have a decreasing trends with altitude. WBI variations of $Q$. suber were modelled by a linear regression, while those of $Q$. canariensis were fitted by quadratic regression. The result implies that the WBI variations of $Q$. suber was too low to have any meaningful relationship $\left(R^{2}=0.09\right)$. In contrast, about $20 \%$ of WBI variations of $Q$. canariensis were explained by elevation gradients.

Overall, PRI tended to decrease as altitude increased. This trend was observed for both Q. suber and Q. canariensis. Although the regression model between RGRI and altitude for $Q$. suber had a slight weak R-squared value than that for Q. canariensis, RGRI of both species showed increasing trends as elevation goes up. Foliar chemical indices, CRI had no statistical relationship with altitude changes. The relationship between ARI and altitude was too weak to find a meaningful relationship. WBI variations of $Q$. canariensis tended to decrease when altitude increases. On the other hand, WBI variations of $Q$. suber had too weak a trend to show a meaningful relationship with environmental gradients. 
Physiological indices of individual trees in Mediterranean woodland estimated using fused airborne LiDAR and hyperspectral imagery

Table 5.2 Regression models between chemical indices and elevation. $x$ is altitude and $\beta_{i}$ $(i=1,2,3)$ is regression coefficients

\begin{tabular}{c||c|c|c|c|c|c}
\hline \multirow{2}{*}{ Species } & \multirow{2}{*}{ Spectral Indices } & \multirow{2}{*}{ Regression model } & \multirow{2}{*}{$R^{2}$} & \multicolumn{4}{|c}{ parameters } \\
\cline { 4 - 7 } & & & $\beta_{1}$ & $\beta_{2}$ & $\beta_{3}$ \\
\hline \hline \multirow{4}{*}{$Q$. suber } & PRI & $y=\beta_{1}+\beta_{2} x+\beta_{3} x^{2}$ & 0.19 & 0.029 & 0.00023 & $2.2 \mathrm{e}-07$ \\
\cline { 2 - 7 } & RGRI & $y=\beta_{1}+\beta_{2} x+\beta_{3} x^{2}$ & 0.16 & 0.87 & 0.00066 & $5.1 \mathrm{e}-07$ \\
\cline { 2 - 7 } & CRI & $\mathrm{n} / \mathrm{a}$ & \multicolumn{4}{|c}{} \\
\cline { 2 - 7 } & ARI & $y=\beta_{1}+\beta_{2} x$ & 0.11 & 0.00072 & $7.6 \mathrm{e}-07$ & $\mathrm{n} / \mathrm{a}$ \\
\cline { 2 - 7 } & WBI & $y=\beta_{1}+\beta_{2} x$ & 0.09 & 1 & $9.9 \mathrm{e}-06$ & $\mathrm{n} / \mathrm{a}$ \\
\hline \multirow{4}{*}{ Q. canariensis } & PRI & $y=\beta_{1}+\beta_{2} x+\beta_{3} x^{2}$ & 0.20 & 0.035 & 0.00022 & $2.3 \mathrm{e}-07$ \\
\cline { 2 - 7 } & RGRI & $y=\beta_{1}+\beta_{2} x+\beta_{3} x^{2}$ & 0.21 & 0.91 & $3.1 \mathrm{e}-05$ & $8.6 \mathrm{e}-07$ \\
\cline { 2 - 7 } & CRI & n/a & \multicolumn{5}{|c}{} \\
\cline { 2 - 7 } & ARI & $y=\beta_{1}+\beta_{2} x+\beta_{3} x^{2}$ & 0.10 & 0.00071 & $6.8 \mathrm{e}-07$ & $1.9 \mathrm{e}-09$ \\
\cline { 2 - 7 } & WBI & $y=\beta_{1}+\beta_{2} x+\beta_{3} x^{2}$ & 0.21 & $-6.9 \mathrm{e}-08$ & $2.1 \mathrm{e}-05$ & 1 \\
\hline
\end{tabular}

\subsection{Discussion}

\subsubsection{Photosynthetic light use efficiency}

PRI is a tool for measuring photosynthetic light use efficiency (LUE) of plants [103]. As light intensity increases, xanthophyll pigments are converted from the epoxidised state (Violaxanthin) to the de-epoxidised state (Antheraxanthin, Zeaxanthin). In addition, PRI shows the relative ratio between carotenoids and chlorophyll, i.e. high PRI indicates that carotenoid contents relative to chlorophyll is low [96]. PRI in Table 5.1 is expressed as a negative value. In this case, decreasing PRI values indicates lower LUE because physiological stress limits leaf photoacclimation [11]. High PRI implies that photons of light absorbed by a leaf are efficiently utilized. Therefore, the regression models show that both $Q$. suber and $Q$. canariensis had high LUE in low altitude. This result had good agreement with previous field studies that PRI decreases with increasing elevation [95, 227, 226]. Filella and Pañuelas [95] reported the same result from two Mediterranean species. They showed that negative signed PRI of Quercus ilex and Rhododendron ferrugineum had an increasing trend with respect to elevation. Richardson and his group studied the spectral reflectance properties of coniferous 
trees [227] and birch trees [226] on environmental gradients using field spectrometer. They showed that PRI tended to decrease as elevation increased. The low PRI value at high altitude may be related with environmental stress. Xanthophyll cycle pigments relative to chlorophyll tends to increase under environment stress [81,226], which may explain decreasing trend of PRI with increasing altitude.

RGRI was originally introduced to estimate anthocyanins contents [104]. However, Red/Green ratio only shows anthocyanins contents relative to chlorophyll contents, so it may not be adequate for direct estimation of anthocyanins [239, 109]. RGRI is an indicator for showing canopy stress, light use efficiency and carbon flux [125]. As shown in Figure 5.7e and 5.8e, RGRI had positive trends with increasing elevation. In other words, canopy stress was relatively higher in higher altitude, while photochemical efficiency tended to decease with increasing elevation. This was in accordance with PRI variations which showed less photosynthetic quantum efficiency in high altitude.

PRI and RGRI imply that high photosynthetic activity was observed in low altitude for both oak species. In addition, PRI and RGRI implies that $Q$. canariensis is more sensitive to environmental gradients than $Q$. suber. It may be related with noticeable species distribution patterns. The study site was located in the valley. Q. canariensis was dominated in the valley bottom, while $Q$. super was populated in the sloped region. Nutrients and water were accumulated in the valley bottom. The soils are deeper and more nutrient rich downslope, therefore less stressful for the plants. In addition, at lower altitude, plants can access water easily compared with higher altitude. High LUE at lower altitude makes sense under these conditions. It also explains why $Q$. canariensis is more sensitive to PRI and RGRI.

\subsubsection{Canopy water contents}

WBI is measuring canopy water content. WBI expressed in Table 5.1 decreases with higher water stress of vegetation. The WBI trends show that $Q$. canariensis is more sensitive 
Physiological indices of individual trees in Mediterranean woodland estimated using fused airborne LiDAR and hyperspectral imagery

to elevation gradients than $Q$. suber. WBI variations of $Q$. canariensis had a decreasing trend with increasing altitude. Although WBI variation of $Q$. suber was also decreased with increasing altitude, it was too weak to show a meaningful trend. Since water stress can cause low photochemical efficiency [56], water stress could explain low PRI and high RGRI values of $Q$. canariensis at higher altitude. Gea et al. analysed xylem variability and growth responses of $Q$. canariensis. They showed that the growth of $Q$. canariensis was limited under water-stressed conditions. Urbieta et al. [252] reported that the abundance of both species increases with precipitation mean rate, and $Q$. canariensis prefers to inhabit near drainage network and streams. Therefore, WBI variations were in accordance with the previous studies that $Q$. canariensis is dominant in areas of the western Mediterranean with the most humid microclimates, and is sensitive to water stress [107, 252]. Specifically, species abundance of $Q$. canariensis was much higher with water availability while $Q$. suber were more abundant with less water availability [252].

\subsubsection{Carotenoids and anthocyanins variations}

CRI had no meaningful trends with respect to environmental gradients, and ARI trends were too weak to have a linkage with environmental variables. In contrast, PRI and RGRI trends imply that carotenoids and anthocyanins contents relative to chlorophyll level had increasing trends with higher altitudes $[111,96]$. This may mean that chlorophyll contents tend to decrease with altitude, so the ratio of carotenoid/chlorophyll and anthocyanin/chlorophyll tends to increase, although carotenoids and anthocyanins may remain stable or weakly increase. These trends are in accordance with previous studies that the decreasing LUE and relative increase of photoprotective pigments in higher altitude [227, 226, 68, 260, 222].

Carotenoids play an important role in photoprotection [248]. The large amount of energy absorbed during photosynthesis due to intensified solar radiation, could produce singlet oxygen molecules, which can damage plant cells. Carotenoids quench the excited 
state of chlorophyll, thus protect plant cells. The role of anthocyanins is rather contentious $[68,166,160]$. A number of papers supported the hypothesis that anthocyanins are involved in UV protection and light attenuation $[219,68,160]$. If the elevation gradient was large enough to affect solar radiation, the increase in altitude would cause an increase in photoprotection pigments [154]. The effect of altitude measured by Blumthaler et al. [35] indicates that solar UV radiation tended to increase 9-24\%, depending upon wavelength, for every $1000 \mathrm{~m}$ increase in altitude. In our study site, solar irradiance cannot have changed dramatically with elevation as the gradient was at most $800 \mathrm{~m}$, therefore, UV radiation changes were too small to have an impact on plant physiological responses.

Nutrient deficiency and water stress could be alternative explanation for these pigment variations. Since many of the lower altitude sites were located in the valley bottom, trees are more likely to access rich nutrients and water compared to higher altitude. Nitrogen deficiency triggers the expression of genes encoding enzymes linked to anthocyanins synthesis $[162,84,36,244]$. In addition, nitrogen deficiency increases the level of the xanthophyll cycle related energy dissipation, thus increasing photoprotective capability and reducing photosynthetic quantum efficiency $[82,81]$. Water stress increases xanthophyll pools and decreases photosynthetic activities [82]. Plant responses with respect to nutrient and water stresses can explain the patterns of spectral indices. PRI and RGRI imply that the light protection pigments increased relative to chlorophyll, and WBI indicates that water stress increases at higher altitude.

\subsubsection{Limitations and future work}

Optical properties of vegetation are influenced by various conditions: geometric characteristics, leaf angle distortion, LAI, vegetation coverage, viewing angles, solar angles etc. [246]. In this study, we try to minimise these effects using the BRDF correction and filtering steps in our framework. The filtering step is designed to remove non-vegetation, shaded 
Physiological indices of individual trees in Mediterranean woodland estimated using fused airborne LiDAR and hyperspectral imagery

and low-LAI pixels. Non-vegetation cover was removed by ITCs and NDVI filter. Pixels outside of ITCs were removed, and NDVI filtered out non-vegetation pixels but some still remained due to registration errors. NDVI is saturated at LAI 3-4 [199, 11], therefore, we can remove pixels if NDVI was less than 0.8 , such that the effects of LAI on spectral indices are minimised. The shade effect was filtered using hillshade ArcGIS built-in function. This function provide illumination conditions using solar positions and surface geometry. This study filtered out illumination below $65 \%$, thus shading effects on spectral indices were also alleviated. Therefore, spectral indices used in this study were not significantly distorted by BRDF, shades and LAI effects.

However, BRDF effects were still visually identifiable in few flight lines. Since NDVI saturated when LAI reaches 3-4 the LAI estimation is still limited. Hillshade function did not take into account optical and structural properties of vegetation, thus filtering shaded pixels might not be enough to ensure reliability of vegetation reflectance. In addition, there are still a number of variables, which could affect optical properties of vegetation [246, 18, 142, 91]. For the accurate assessment for spectral indices, we need to simulate the effects of BRDF, shadows and LAI on spectral indices using a radiative transfer model [246, 18, 142, 91]. For instance, Suarez et al. [246] used a radiative transfer model to assess the role of various optical parameters on spectral indices. In this study, PRI is robust against the BRDF effect, but sensitive to LAI and soil background. Other indices, however, have not been evaluated properly yet. Measuring reliability of spectral indices requires a sophisticated radiative transfer model with ground truth canopy spectra in various experimental settings, which is beyond the scope of this study.

As shown in Chapter 3 and 4, MCNCP-RNC algorithm tends to over-segment ITCs. Over-segmentation of ITCs contributed to errors because a single treetop pixel per ITC is used to examine the variations of spectral indices. Over-segmented ITCs mean that several observations in a spectral index plot in Figure 5.7 and 5.8 may have originated from a single 
tree. More accurate ITC delineation algorithm is needed to get more reliable results from spectral indices.

Two oak species were mapped by SVM with visual inspection. Although $Q$. suber and $Q$. canariensis were dominant in the study site, a few other species were also present. Since we did not have field plots across the study area, it is hard to evaluate how many other species were included in our analysis. The spectral indices could be sensitive to species, so it may explain low correlation between environmental gradient and physiological indices [103]. This effect could be alleviated if we can include or filter out other species in the study area. However, this requires field work to sample ground truth in the study area.

This study is preliminary work exploring the potential of our data processing framework for analysing ITC-level physiological responses. Since we do not have ground truth, our analysis may be or may not be true in this study site. However, our results had good agreement with previous studies that photochemical efficiency tended to decrease with increasing elevation. Since our site is located in a valley, it makes sense that low altitude had more nutrients and nitrogen, which are related with productivity of trees [226]. In this context, our analysis had meaningful outcomes, although validation and calibration of our regression models with field measurements were not conducted. We leave this for future work. With the linkage of ground truth data, we can directly find a statistical relationship between leaf chemistry, leaf spectra and canopy spectra in the spectranomics [17] approach. Asner and his group investigated the linkage between 23 foliar chemical traits and spectral information $[17,18,16,14,23]$. This spectranomics approach can monitor canopy stress caused by drought [10] and examine taxonomic diversity [23]. If we have enough field measured samples of the 23 chemical traits and spectra, spectranomics can be applied to our dataset as well. Specifically, the combination of our ITC-level physiological index analysis and spectranomics could provide information about individual tree stress and carbon storage. 
Physiological indices of individual trees in Mediterranean woodland estimated using fused airborne LiDAR and hyperspectral imagery

\subsection{Concluding remarks}

The research has developed a workflow of using spectral indices at ITC level over about $35 \mathrm{~km}^{2}$ of woodland in southern Spain. This preliminary study shows the promising application of the fusion of LiDAR and hyperspectral imagery. Spectral indices of ITCs obtained from hyperspectral images could monitor physiological variations of two oak species with respect to environmental gradients. The decreasing LUE with increasing altitude was captured by spectral indices at ITC level. Specifically, $20 \%$ of LUE was influenced by altitudinal gradients. Moreover, our method captured that Q. canariensis is more sensitive to environmental stresses than $Q$. suber. Our results were in agreement with previous studies on physiological variations of plant species with altitudinal gradients, and biogeography of $Q$. canariensis and $Q$. suber. However, further validation using ground truth is still required to confirm our analysis results. 


\section{Chapter 6}

\section{General discussion}

The main objective of this thesis is to develop a series of technical solutions for using airborne multi-sensor approaches in ecological research. Namely, to:

- Co-align multi-sensor airborne remote sensing: LiDAR, hyperspectral and aerial photographs in a wooded landscape;

- Develop a 3D individual tree crown delineation algorithm using LiDAR and hyperspectral imagery;

- Design a novel protocol of airborne multi-sensor remote sensing to map species in both pixel and ITC levels;

- Develop a workflow for multi-sensor airborne remote sensing to monitor plant physiological responses with respect to environmental gradients at ITC level.

Specifically, this thesis puts emphasis on technical issues of airborne hyperspectral imagery and LiDAR. The NGF-Curv method we developed can co-align images taken from hyperspectral imagery and LiDAR in a semi-automatic way (Chapter 2). We could then delineate ITCs with the MCNCP-RNC approach from the co-aligned LiDAR and hyperspectral imagery. MCNCP-RNC showed better ITC delineation than TIFFS, which was 
based on the CHM. Since MCNCP-RNC identifies ITC directly from the LiDAR point cloud, it is not influenced by interpolation related artefacts, therefore more accurate ITC parameter estimation is available (Chapter3). Both pixel-level and ITC-level species delineation was conducted by the workflow designed for multi-sensor imaging. This workflow ensures coalignment of multi-sensor imagery using NGF-Curv, delineating ITCs using MCNCP-RNC. Using 20 principal components from rPCA, our ISTA-R method achieved overall $89.1 \%$ pixel-level accuracy, and 65.8\% ITC-level accuracy (Chapter 4). We modified the workflow for analysing physiological responses of two oak species (Q. canariensis and $Q$. suber) with respect to environmental gradients in a Mediterranean woodland. We used five spectral indices, which reflect physiological changes of the canopy. These indices showed that both species decreased photochemical efficiency with increasing altitude and Q. canariensis was more sensitive to environmental gradients. These were in accordance with previous studies, thus showing the efficiency of using multi-sensor imagery for monitoring physiological stresses of canopy trees (Chapter 5).

\subsection{Nonparametric image registration of airborne LiDAR, hyperspectral and photographic imagery of wooded land- scapes}

The advantage of using an airborne multi-sensor approach is that each sensor can provide different features, which can be used to infer details about ecological processes $[15,17]$. However, images acquired from different sensors have different relative rotation, translation, and scale mismatches, requiring correction before merging.

Image registration correction involves transforming a template image $T$ using a transformation $\phi$ so that it aligns with a reference image $R$. The NGF-Curv method $[118,98]$ is a non-parametric image registration correction, i.e. $\phi$ is not defined by a set of explicit 
6.1 Nonparametric image registration of airborne LiDAR, hyperspectral and photographic imagery of wooded landscapes

translation, rotation, or affine transformation parameters. The performances of the NGF-Curv shows that non-linear distortion caused by topography can be adjusted, and misalignment caused by georeferencing errors can be corrected using LiDAR intensity imagery.

Another benefit of the NGF-Curv algorithm is that it needs little prior knowledge of correspondences between template and reference images. If the images are georeferenced, then the NGF-Curv can be implemented directly. Alternatively, the flight position can be used as the centre of each image, and determine approximate geographic boundaries for each image.

The NGF-Curv can handle co-alignment of images obtained from different sensors, as it measures the similarity by comparing two images. If we assume that intensity changes appear at corresponding positions even for images taken from different sensors, then intensity changes indicated by the image gradient could quantify the similarity between the images [118]. In addition, the curvature regularisation reduces ill-posedness of our registration method, such that it ensures that our registration method is meaningful [98].

\subsubsection{Limitation}

Although the NGF-Curv method successfully co-aligned LiDAR, hyperspectral and aerial photographs in a Spanish woodland, a few limitations remain. In theory, intensity based methods such as NGF-Curv, can be fully automatic. However, in practice this is only available for the NGF-Curv when all parameters are fixed. The NGF-Curv has two important parameters: a noise parameter $\eta$ that determines meaningful magnitude of gradients in the NGF similarity measure and a regularisation parameter $\alpha$ that balances the NGF similarity measure and curvature regularisation. In Chapter 2, we used a trial-and-error approach to find and fix these parameters. Although the experiments with fixed parameters showed reasonable performance, they caused mis-alignment at the edges of the image. Therefore, NGF-Curv is 
curently semi-automatic because images with significant misalignment at the edge should be corrected by parameter tuning.

Since NGF assumes that intensity changes appear at corresponding positions, it is very sensitive to illumination effects. Hyperspectral and aerial photographs often contain shaded or clouded pixels because they measure backscattered solar energy. In contrast, LiDAR data does not have much illumination artefacts as it scans the surface using a monochromatic light beam. Therefore, the registration process between shaded LiDAR and hyperspectral images could be biased as NGF compares only gradient information between the images.

In principle, curvature regularisation makes the problem well-posed, ensuring NGFCurv has a meaningful solution. However, it is challenging to remove all local minima in practice, hence the NGF-Curv is still influenced by the initial alignment of images. Futhermore, curvature regularisation penalises affine transformation due to its boundary condition, requiring parametric affine registration to be applied prior to NGF-Curv method for generic applications [123, 194].

\subsubsection{Future work}

NGF-Curv is semi-automatic at its current stage. Further work is required to establish an automatic way to determine parameters, thus making the NGF-Curv algorithm more robust. NGF-Curv penalises affine transformation because of its Dirichlet or Neumann boundary conditions [123]. Therefore, affine registration is a pre-requisite for generic applications. Further work is required to improve curvature regularisation, thus making the NGF-Curv algorithm standalone. Since NGF-Curv is sensitive to illumination effects, developing shade removal techniques or a new similarity measure robust to illumination effects is needed [97]. 
6.2 3D individual tree segmentation of integrated airborne LiDAR and optical imagery using normalised cut with priors

\subsection{D individual tree segmentation of integrated airborne LiDAR and optical imagery using normalised cut with priors}

Airborne LiDAR produces a 3D point cloud indicating where laser pulses from the sensor have reflected off leaves, branches and the forest floor, making it possible to map individual trees over tens of thousands of hectares. Airborne optical imagery can be used to estimate the physical and chemical properties of canopies, and when used alongside LiDAR - map these properties down to the level of individual tree crowns [78].

We developed the MCNCP-RNC algorithm to delineate ITCs from both LiDAR and hyperspectral imagery [237, 133]. The major advantage of the MCNCP-RNC algorithm is that graph weights can be defined using any optical imagery with LiDAR, thus providing a framework for fusing images taken from different sensors. MCNCP-RNC can incorporate priors, such that the graph cut algorithm is constrained by the locations of priors [133]. In addition, MCNCP-RNC could find understory trees when LiDAR point density is dense enough to represent internal tree structures, as the RNC step checks the further separability of each ITC. We tested MCNCP-RNC algorithm in three different datasets: Italian, English and Benchmark datasets. MCNCP-RNC algorithm showed superior performance compared to TIFFS [60] in all three datasets. Specifically, optical imagery alongside LiDAR could improve ITC delineation if the resolution of the imagery is sufficiently high. In addition, MCNCP-RNC provides 3D information of ITCs, which is particularly useful to estimate various ITC parameters, including crown area, crown shape and tree height. These parameters may help to detect species and predict forest biomass more accurately. 


\subsubsection{Limitation}

The tree detection accuracy of MCNCP-RNC was strongly influenced by priors. Since RNC step checks the further separability of each ITC, MCNCP-RNC can delineate more trees than predicted by the number of local maxima. However, MCNCP-RNC has limited performance at merging ITCs predicted in the prior because it is strongly constrained by it [133]. MCNCP-RNC could merge oversegmented trees in the limited condition that two priors were very closely located. This is because the output of MCNCP-RNC must meet the correlation constraint of the prior.

MCNCP-RNC found a few additional understory trees compared to CHM based methods. However, the performance of MCNCP-RNC for identifying understory trees was very limited due to the fixed bandwidth parameters, which could not represent point distribution of each ITC.

Although MCNCP-RNC was computationally faster than RNC, it is still computationally heavy. If the number of trees predicted by priors is large, computation of equivalent numbers of eigenvectors is needed and they are usually dense. For example, if we have thousands of local maxima, then we need to compute the first thousand of eigenvectors to delineate ITCs.

Hyperspectral imagery can improve ITC delineation, however, it also costs more oversegmentation. It may be related with illumination artefacts or registration errors $[78,250]$. In particular, the optimal number of principal components needed for ITC delineation have not been investigated. The first component can lead to inaccurate ITC delineation because it contains illumination information [250]. However, the first principal component also plays a key role in species classification, which may help ITC delineation. 
6.2 3D individual tree segmentation of integrated airborne LiDAR and optical imagery using normalised cut with priors

\subsubsection{Future work}

A new model is required to resolve problems of MCNCP-RNC. Advanced methods for the graph partitioning problem based on $\ell^{1}$ optimisation technique provide a more accurate and fast solution than MCNCP-RNC [49, 41, 42].

In the normalised cut framework [237, 133], the solutions of graph cut are approximated using spectral clustering, which is $\ell^{2}$ relaxation of original graph cut problems [256]. Spectral clustering resolves the generalised eigenvalue problem, then computes the second smallest and subsequent eigenvectors. We refer to Section 2 in Chapter 3 for further details.

Buhler and Hein [49] recently suggested a tight relaxation of Cheeger ratio cut called " $p$-spectral clustering". They proved that $p \rightarrow 1$, it solves the exact graph cut problem. For example, $\ell^{1}$ relaxation gives the exact solution of Cheeger ratio cut problem [57]. This $\ell^{1}$ relaxation can be reformulated as total variation problem [41, 42]. Under this setting, we can apply a domain decomposition technique, such as the subspace correction method [85] to reduce memory demand. Another benefit of the $\ell^{1}$ relaxation is that we can incorporate soft priors with few modifications, therefore, oversegmented trees could be merged together.

Improved understory searching algorithm is required to achieve more accurate ITC delineation. This can be resolved by examining LiDAR vertical profiles statistics of each canopy tree [86]. This method may provide a useful parameter to guide the RNC algorithm to delineate understory trees. In addition, using Full-waveform LiDAR instead of discrete return LiDAR can also improve ITC segmentation as it could provide more details of internal structures.

Hyperspectral imagery can guide ITC delineation if the resolution of hyperspectral imagery is high enough. However, the optimal number of principal components of hyperspectral imagery for ITC delineation is still unknown. The first principal component can cause ITC delineation errors because it includes illumination information [250]. However, the first principal component is important for species classification at pixel level, so it may help to 
improve ITC delineation as well. Further study is required to decide the optimal number of principal components for ITC delineation.

\subsection{Individual tree species classification from airborne multi- sensor imagery}

Mapping ITCs and species using multi-sensor imagery is a powerful technique to analyse patterns of species distribution, monitor exotic and invasive species and calculate very precise forest carbon storage $[17,16]$. In Chapter 4 , we apply methods developed in chapter 2 and 3 , and design a workflow to map individual tree crowns and identify species using multi-sensor imagery.

We assumed that only radiometric correction and georeferencing of LiDAR and hyperpspetral imagery were performed in the pre-processing step. Therefore, our scheme can be applied to any multi-sensor imaging with LiDAR and optical imagery. In addition, we introduced robust PCA [53] for feature extraction, such that species classification accuracy was improved. Species identification and mapping was conducted at two different levels. First, we compared pixel-level species classification results using confusion matrix, then we analyse ITC-level species classification results.

Overall, our schematic work flow achieved $89.1 \%$ overall classification accuracy at pixel level, while only $65.8 \%$ overall accuracy was achieved in ITC-level species classification. The NewFor validation software used for assessing ITC delineation was not performed well in our datasets because field measured tree locations were inaccurate. ITC-level species classification could be more accurate than the results we have. Alternatively, visual inspection was also conducted to evaluate species classification results. The visual analysis indicates that ITC-level species map of Larix decidua, Acer pseudoplatanus, Betula spp. and 
Fraxinus excelsior were in agreement with ground truth, while other species seemed to be oversegmented.

\subsubsection{Limitation}

Although our workflow successfully classified species with $89.1 \%$ accuracy at pixel level, ITC-level species classification is still suboptimal. Since the validation software we used was sensitive to initial positioning of ground truth, it is hard to validate the actual performance of our workflow. Even if visual analysis shows that segmented tree species had good agreement with our ground truth, it is not precise enough to show the validity of our ITC-level species classification or suggest further work. Therefore, more thorough validation should be conducted with more accurately located ground truth, such that ITC level errors can be examined precisely.

We classified species from hyperspectral imagery only. LiDAR derived features such as LiDAR intensity imagery or CHM could improve species classification further [74, 122, 79]. The role of LiDAR derived features on species classification is a missing part of our workflow.

The visual analysis showed that some large tree crowns of Fagus sylvastica and Quercus robur were over-segmented by our delineation algorithm. The improvement of the NGF-Curv method could improve the species classification at ITC-level. As we mentioned in Chapter 3, MCNCP-RNC has difficulty to merge two priors together. The improved version of MCNCPRNC could show better ITC-level species classification. MCNCP-RNC uses information taken from both LiDAR and hyperspectral imagery. Although we ensured co-alignment of LiDAR and hyperspectral imagery using NGF-Curv registration in Chapter 2, mismatches could remain to some extent, therefore, these may cause distortion of our ITC-level species classification results. Moreover, the role of hyperspectral imagery on ITC delineation is still unclear. The first principal component of hyperspectral imagery can lead to inaccurate ITC 
delineation because it contains illumination information [250]. However, the first principal component also plays a key role in species classification, which may help ITC delineation.

\subsubsection{Future work}

Precisely geo-coded ground truth is needed to validate our models either in the same or different field site. The validation in Chapter 4 showed the effectiveness of the model in the limited condition that ground truth was inaccurate. Access to validation data will provide more ways to improve our workflow.

In this study, we only used hyperspectral imagery for species classification. LiDAR may provide further features, which may play an important role in species classification [122]. For example, LiDAR intensity data were not calibrated because of non-linearity caused by the automatic gain control system, so it cannot be used for species classiication $[149,156]$. If we can offset the automatic gain control system, then the LiDAR intensity data may provide additional features, which can guide species classification. However, LiDAR may fail to provide any further meaningful data as unlike hyperspectral imagery, LiDAR does not contain shades because it is an active sensor, i.e. sending and measuring signal by a transceiver. Therefore, further study is needed to investigate the role of LiDAR derived features on species classification.

Finally, the improvements of NGF-Curv and MCNCP-RNC methods in Chapter 2 and 3 will help to classify species more accurately due to accurate registration and ITC delineation. 
6.4 Physiological indices of individual trees in Mediterranean woodland estimated using fused airborne LiDAR and hyperspectral imagery

\subsection{Physiological indices of individual trees in Mediterranean}

\section{woodland estimated using fused airborne LiDAR and}

\section{hyperspectral imagery}

Canopy physiology is a key to understand energy flux, nutrient cycling and species diversity of forests. Monitoring canopy physiology using multi-sensor imaging could help to assess the impacts of environmental and taxonomic diversity on plant physiology $[11,17]$. We redesigned the workflow developed in Chapter 4 to monitor canopy physiology. In this study, we examined five ITC-level spectral indices of canopy physiology [102, 104, 111, 112, 215]. We tested our workflow in a Mediterranean woodland, where two oak species (Quercus suber and Quercus canariensis) were dominant. We compared these indices with environmental variables: elevation, slope and aspect.

The analysis shows that PRI of both species decreased at higher altitudes, while Red/Green ratio index increased with elevation. These trends are coherent with previous field studies showing that LUE decreases as elevation increases. The regression fitting model implies that $20 \%$ of LUE was influenced by elevation gradients. WBI of Quercus canariensis had a decreasing trend, which is in accordance with previous studies on Mediterranean ecosystems $[95,107,252]$. This study demonstrates the potential of multi-sensor imaging for monitoring Mediterranean ecosystem at ITC level.

\subsubsection{Limitation}

Optical properties of vegetation are influenced by various conditions: geometric characteristics, leaf angle distortion, LAI, vegetation coverage, viewing angles, solar angles etc. $[71,246]$. Although pre-processing and filtering steps minimise the effect of BRDF, LAI, and shades, they may remain to some extent, thus causing errors. BRDF effects were still visually identifiable in a few flight lines. NDVI saturated as LAI reaches $3-4$, so the LAI 
estimation using NDVI had a limitation. The hillshade function did not consider various optical and structural properties of vegetation. Shade effects may remain in hyperspectral imagery.

Since we did not collect data, the calibration of spectral indices with field data was not available. Although Quercus canariensis and Quercus suber can be identified visually on hyperspectral imagery, species classification might be biased because other minority species can also be included in our analysis. The lack of ground truth indicates that we cannot evaluate the accuracy of spectral indices directly.

\subsubsection{Future work}

The assessment of spectral indices with different optical parameters is required to evaluate the reliability of physiological parameters derived from spectral indices. A sophisticated radiative transfer model is needed to assess the role of various optical parameters on spectral indices. The radiative transfer model could help to interpret spectral indices under different optical properties. For example, Suarez et al. [246] simulated the role of various optical parameters on spectral indices using a radiative transfer model. They found that PRI is sensitive to soil background and LAI, while it is robust against the BRDF effect.

The lack of ground truth causes inaccurate species classification in our workflow. Although our study site was dominated by only two oak species, there were a few other species. Since spectral indices of canopy physiology can be dependent on species, it could bias the result. Field work is required to include or exclude other species from our analysis. Since we do not have ground truth of canopy physiology or optical properties, our analysis may be or may not be true in this study site. Although our results are in accordance with previous studies on Mediterranean ecosystem, field data is required to confirm our findings.

With the collection of leaf spectra and chemical traits of each species, spectranomics recently developed by Asner's group can be included in our workflow [17]. This spectra- 
nomics approach predicts leaf chemistry at canopy level, such that taxonomic diversity can be monitored [18]. The combination of our ITC-level physiology indices and spectranomics could provide information about individual tree stress more accurately.

\subsection{Concluding remark}

Airborne multi-sensor imaging could be a key to understand detailed ecological traits in a large scale. However, designing techniques for inferring ecological traits from multi-sensor imaging are still in their infancy. In this thesis, we developed technical solutions for mapping species and monitoring physiological stress of canpy trees using airborne multi-sensor imagery. The results in this thesis show that airborne multi-sensor imaging can provide very detailed species and physiological responses of canopy trees. It can be used to monitor deforestation, measure species specific forest biomass, and understand patterns of species competition and distribution. If we repeat airborne survey regularly, then the changes of forest coverage can be easily estimated by comparing it with the previous survey. As shown in Chapter 4 and 5, our workflow can be used to classify species at ITC-level, therefore, species-specific biomass estimation can be derived from LiDAR and hyperspectral imagery. In addition, our workflow can be used to monitor species distribution and competition. For example, in Chaper 5, our pre-liminary work showed that Quercus canariensis was polulated in the valley bottom, while Quercus suber preferred sloped region. In this context, airborne multi-sensor imaging would contribute to resolving some ecological issues which have not been answered by conventional ecological methods. 



\section{References}

[1] Abshire, J. B., Sun, X., Riris, H., Sirota, J. M., McGarry, J. F., Palm, S., Yi, D., and Liiva, P. (2005). Geoscience laser altimeter system (GLAS) on the ICESat mission: on-orbit measurement performance. Geophysical Research Letters, 32(21).

[2] Adams, J. B. and Gillespie, A. R. (2006). Remote sensing of landscapes with spectral images: a physical modeling approach. Cambridge University Press.

[3] Alonzo, M., Bookhagen, B., and Roberts, D. A. (2014). Urban tree species mapping using hyperspectral and LiDAR data fusion. Remote Sensing of Environment, 148:70-83.

[4] Aronson, J., Pereira, J. S., and Pausas, J. G. (2012). Cork oak woodlands on the edge: ecology, adaptive management, and restoration. Island Press.

[5] Ascher, U., Haber, E., and Huang, H. (2006). On effective methods for implicit piecewise smooth surface recovery. SIAM Journal on Scientific Computing, 28(1):339-358.

[6] Asner, G., Martin, R., Knapp, D., Kennedy-Bowdoin, T., and Kellner, J. (2012a). Impacts of the 2010 Amazon drought on forest structure and function using CAO AToMS. In $A G U$ Fall Meeting Abstracts, volume 1, page 04.

[7] Asner, G. P. (1998). Biophysical and biochemical sources of variability in canopy reflectance. Remote Sensing of Environment, 64(3):234-253.

[8] Asner, G. P. (2008). Hyperspectral remote sensing of canopy chemistry, physiology, and biodiversity in tropical rainforests. In Kalacska, M. and Sanchez-Azofeifa, G. A., editors, Hyperspectral remote sensing of tropical and sub-tropical forests, pages 261-296. CRC Press.

[9] Asner, G. P. (2009). Tropical forest carbon assessment: integrating satellite and airborne mapping approaches. Environmental Research Letters, 4(3):034009.

[10] Asner, G. P. and Alencar, A. (2010). Drought impacts on the Amazon forest: the remote sensing perspective. New Phytologist, 187(3):569-578.

[11] Asner, G. P., Carlson, K. M., and Martin, R. E. (2005). Substrate age and precipitation effects on Hawaiian forest canopies from spaceborne imaging spectroscopy. Remote Sensing of Environment, 98(4):457-467.

[12] Asner, G. P., Hughes, R., Vitousek, P., Knapp, D., Kennedy-Bowdoin, T., Boardman, J., Martin, R., Eastwood, M., and Green, R. (2008a). Invasive plants transform the threedimensional structure of rain forests. Proceedings of the National Academy of Sciences, 105(11):4519-4523. 
[13] Asner, G. P., Jones, M. O., Martin, R. E., Knapp, D. E., and Hughes, R. F. (2008b). Remote sensing of native and invasive species in Hawaiian forests. Remote Sensing of Environment, 112(5):1912-1926.

[14] Asner, G. P., Knapp, D., Balaji, A., and Páez-Acosta, G. (2009a). Automated mapping of tropical deforestation and forest degradation: CLASlite. Journal of Applied Remote Sensing, 3(1):033543-033543.

[15] Asner, G. P., Knapp, D. E., Kennedy-Bowdoin, T., Jones, M. O., Martin, R. E., Boardman, J., and Field, C. B. (2007). Carnegie airborne observatory: in-flight fusion of hyperspectral imaging and waveform light detection and ranging for three-dimensional studies of ecosystems. Journal of Applied Remote Sensing, 1(1):013536-013536.

[16] Asner, G. P., Knapp, D. E., Kennedy-Bowdoin, T., Jones, M. O., Martin, R. E., Boardman, J., and Hughes, R. F. (2008c). Invasive species detection in Hawaiian rainforests using airborne imaging spectroscopy and LiDAR. Remote Sensing of Environment, 112(5):1942-1955.

[17] Asner, G. P. and Martin, R. E. (2008a). Airborne spectranomics: mapping canopy chemical and taxonomic diversity in tropical forests. Frontiers in Ecology and the Environment, 7(5):269-276.

[18] Asner, G. P. and Martin, R. E. (2008b). Spectral and chemical analysis of tropical forests: scaling from leaf to canopy levels. Remote Sensing of Environment, 112(10):3958-3970.

[19] Asner, G. P. and Martin, R. E. (2011). Canopy phylogenetic, chemical and spectral assembly in a lowland Amazonian forest. New Phytologist, 189(4):999-1012.

[20] Asner, G. P., Martin, R. E., Ford, A. J., Metcalfe, D. J., and Liddell, M. J. (2009b). Leaf chemical and spectral diversity in Australian tropical forests. Ecological Applications, 19(1):236-253.

[21] Asner, G. P., Martin, R. E., Knapp, D. E., Tupayachi, R., Anderson, C., Carranza, L., Martinez, P., Houcheime, M., Sinca, F., and Weiss, P. (2011a). Spectroscopy of canopy chemicals in humid tropical forests. Remote Sensing of Environment, 115(12):3587-3598.

[22] Asner, G. P., Martin, R. E., and Suhaili, A. B. (2012b). Sources of canopy chemical and spectral diversity in lowland Bornean forest. Ecosystems, 15(3):504-517.

[23] Asner, G. P., Martin, R. E., Tupayachi, R., Emerson, R., Martinez, P., Sinca, F., Powell, G. V., Wright, S. J., and Lugo, A. E. (2011b). Taxonomy and remote sensing of leaf mass per area (LMA) in humid tropical forests. Ecological Applications, 21(1):85-98.

[24] Baccini, A., Goetz, S., Walker, W., Laporte, N., Sun, M., Sulla-Menashe, D., Hackler, J., Beck, P., Dubayah, R., Friedl, M., et al. (2012). Estimated carbon dioxide emissions from tropical deforestation improved by carbon-density maps. Nature Climate Change, 2(3):182-185.

[25] Baccini, A., Laporte, N., Goetz, S., Sun, M., and Dong, H. (2008). A first map of tropical Africa's above-ground biomass derived from satellite imagery. Environmental Research Letters, 3(4):045011. 
[26] Baghdadi, N., Le Maire, G., Fayad, I., Bailly, J. S., Nouvellon, Y., Lemos, C., and Hakamada, R. (2014). Testing different methods of forest height and aboveground biomass estimations from ICESat/GLAS data in Eucalyptus plantations in Brazil. Selected Topics in Applied Earth Observations and Remote Sensing, IEEE Journal of, 7(1):290-299.

[27] Baldeck, C. A. and Asner, G. P. (2014). Improving remote species identification through efficient training data collection. Remote Sensing, 6(4):2682-2698.

[28] Ballhorn, U., Jubanski, J., and Siegert, F. (2011). ICESat/GLAS data as a measurement tool for peatland topography and peat swamp forest biomass in Kalimantan, Indonesia. Remote Sensing, 3(9):1957-1982.

[29] Barton, C. and North, P. (2001). Remote sensing of canopy light use efficiency using the photochemical reflectance index: model and sensitivity analysis. Remote Sensing of Environment, 78(3):264-273.

[30] Bay, H., Ess, A., Tuytelaars, T., and Van Gool, L. (2008). Speeded-up robust features (SURF). Computer vision and image understanding, 110(3):346-359.

[31] Beer, C., Reichstein, M., Tomelleri, E., Ciais, P., Jung, M., Carvalhais, N., Rödenbeck, C., Arain, M. A., Baldocchi, D., Bonan, G. B., et al. (2010). Terrestrial gross carbon dioxide uptake: global distribution and covariation with climate. Science, 329(5993):834838.

[32] Beg, M., Miller, M., Trouvé, A., and Younes, L. (2005). Computing large deformation metric mappings via geodesic flows of diffeomorphisms. International Journal of Computer Vision, 61(2):139-157.

[33] Bentoutou, Y., Taleb, N., Kpalma, K., and Ronsin, J. (2005). An automatic image registration for applications in remote sensing. Geoscience and Remote Sensing, IEEE Transactions on, 43(9):2127-2137.

[34] Blair, J. B., Coyle, D. B., Bufton, J. L., and Harding, D. J. (1994). Optimization of an airborne laser altimeter for remote sensing of vegetation and tree canopies. In Geoscience and Remote Sensing Symposium, 1994. IGARSS'94. Surface and Atmospheric Remote Sensing: Technologies, Data Analysis and Interpretation., International, volume 2, pages 939-941. IEEE.

[35] Blumthaler, M., Ambach, W., and Ellinger, R. (1997). Increase in solar UV radiation with altitude. Journal of Photochemistry and Photobiology B: Biology, 39(2):130-134.

[36] Bongue-Bartelsman, M. and Phillips, D. (1995). Nitrogen stress regulates gene expression of enzymes in the flavonoid biosynthetic pathway of tomato. Plant Physiology and Biochemistry, 33(5):539-546.

[37] Boudreau, J., Nelson, R. F., Margolis, H. A., Beaudoin, A., Guindon, L., and Kimes, D. S. (2008). Regional aboveground forest biomass using airborne and spaceborne LiDAR in Québec. Remote Sensing of Environment, 112(10):3876-3890.

[38] Brandtberg, T. (2007). Classifying individual tree species under leaf-off and leaf-on conditions using airborne LiDAR. ISPRS Journal of Photogrammetry and Remote Sensing, 61(5):325-340. 
[39] Brandtberg, T., Warner, T. A., Landenberger, R. E., and McGraw, J. B. (2003). Detection and analysis of individual leaf-off tree crowns in small footprint, high sampling density LiDAR data from the eastern deciduous forest in North America. Remote Sensing of Environment, 85(3):290-303.

[40] Breidenbach, J., Næsset, E., Lien, V., Gobakken, T., and Solberg, S. (2010). Prediction of species specific forest inventory attributes using a nonparametric semi-individual tree crown approach based on fused airborne laser scanning and multispectral data. Remote Sensing of Environment, 114(4):911-924.

[41] Bresson, X. and Szlam, A. D. (2010). Total variation, cheeger cuts. In Proceedings of the 27th International Conference on Machine Learning (ICML-10), pages 1039-1046.

[42] Bresson, X., Tai, X.-C., Chan, T. F., and Szlam, A. (2014). Multi-class Transductive Learning Based on $\ell^{1}$ Relaxations of Cheeger Cut and Mumford-Shah-Potts Model. Journal of Mathematical Imaging and Vision, 49(1):191-201.

[43] Bro-Nielsen, M. and Gramkow, C. (1996). Fast fluid registration of medical images. In Visualization in Biomedical Computing, pages 265-276. Springer.

[44] Brown, L. (1992). A survey of image registration techniques. ACM computing surveys (CSUR), 24(4):325-376.

[45] Brown, M. E., Pinzón, J. E., Didan, K., Morisette, J. T., and Tucker, C. J. (2006). Evaluation of the consistency of long-term NDVI time series derived from AVHRR, SPOTvegetation, SeaWiFS, MODIS, and Landsat ETM+ sensors. Geoscience and Remote Sensing, IEEE Transactions on, 44(7):1787-1793.

[46] Brown, S. (2002). Measuring carbon in forests: current status and future challenges. Environmental pollution, 116(3):363-372.

[47] Brunner, D., Lemoine, G., and Bruzzone, L. (2010). Earthquake damage assessment of buildings using VHR optical and SAR imagery. Geoscience and Remote Sensing, IEEE Transactions on, 48(5):2403-2420.

[48] Bueger, M. (1987). Geometry I. Berlin: Springer.

[49] Bühler, T. and Hein, M. (2009). Spectral clustering based on the graph p-Laplacian. In Proceedings of the 26th Annual International Conference on Machine Learning, pages 81-88. ACM.

[50] Bunting, P., Lucas, R., and Labrosse, F. (2008). An area based technique for imageto-image registration of multi-modal remote sensing data. In Geoscience and Remote Sensing Symposium, 2008. IGARSS 2008. IEEE International, volume 5, pages V-212. IEEE.

[51] Burger, M., Modersitzki, J., and Ruthotto, L. (2013). A hyperelastic regularization energy for image registration. SIAM Journal on Scientific Computing, 35(1):B132-B148.

[52] Butt, N., Campbell, G., Malhi, Y., Morecroft, M., Fenn, K., and Thomas, M. (2009). Initial Results from Establishment of a Long-term Broadleaf Monitoring Plot at Wytham Woods, Oxford, UK. University of Oxford Report. 
[53] Candès, E. J., Li, X., Ma, Y., and Wright, J. (2011). Robust principal component analysis? Journal of the ACM (JACM), 58(3):11.

[54] Castro-Esau, K. L., Sánchez-Azofeifa, G. A., Rivard, B., Wright, S. J., and Quesada, M. (2006). Variability in leaf optical properties of Mesoamerican trees and the potential for species classification. American Journal of Botany, 93(4):517-530.

[55] Chang, C.-C. and Lin, C.-J. (2011). LIBSVM: A library for support vector machines. ACM Transactions on Intelligent Systems and Technology, 2:27:1-27:27.

[56] Chaves, M. M., Pereira, J. S., Maroco, J., Rodrigues, M. L., Ricardo, C. P. P., Osório, M. L., Carvalho, I., Faria, T., and Pinheiro, C. (2002). How plants cope with water stress in the field? Photosynthesis and growth. Annals of botany, 89(7):907-916.

[57] Cheeger, J. (1970). A lower bound for the smallest eigenvalue of the Laplacian. Problems in analysis, 625:195-199.

[58] Chen, H., Arora, M., and Varshney, P. (2003a). Mutual information-based image registration for remote sensing data. International Journal of Remote Sensing, 24(18):3701-3706.

[59] Chen, H.-M., Varshney, P. K., and Arora, M. K. (2003b). Performance of mutual information similarity measure for registration of multitemporal remote sensing images. Geoscience and Remote Sensing, IEEE Transactions on, 41(11):2445-2454.

[60] Chen, Q., Baldocchi, D., Gong, P., and Kelly, M. (2006). Isolating individual trees in a savanna woodland using small footprint LiDAR data. Photogrammetric Engineering \& Remote Sensing, 72(8):923-932.

[61] Chen, Q., Gong, P., Baldocchi, D., and Xie, G. (2007). Filtering airborne laser scanning data with morphological methods. Photogrammetric Engineering \& Remote Sensing, 73(2):175-185.

[62] Chiang, M., Leow, A., Klunder, A., Dutton, R., Barysheva, M., Rose, S., McMahon, K., Zubicaray, G. D., Toga, A., and Thompson, P. (2008). Fluid registration of diffusion tensor images using information theory. Medical Imaging, IEEE Transactions on, 27(4):442-456.

[63] Cho, M. A., Debba, P., Mathieu, R., Naidoo, L., Van Aardt, J., and Asner, G. P. (2010). Improving discrimination of savanna tree species through a multiple-endmember spectral angle mapper approach: canopy-level analysis. Geoscience and Remote Sensing, IEEE Transactions on, 48(11):4133-4142.

[64] Cho, M. A., Skidmore, A., Corsi, F., Van Wieren, S. E., and Sobhan, I. (2007). Estimation of green grass/herb biomass from airborne hyperspectral imagery using spectral indices and partial least squares regression. International Journal of Applied Earth Observation and Geoinformation, 9(4):414-424.

[65] Christensen, G. (1994). Deformable shape models for anatomy. PhD thesis, Washington University Saint Louis, Mississippi.

[66] Clark, D. B., Castro, C. S., Alvarado, L. D. A., and Read, J. M. (2004). Quantifying mortality of tropical rain forest trees using high-spatial-resolution satellite data. Ecology Letters, 7(1):52-59. 
[67] Clark, M. L., Roberts, D. A., and Clark, D. B. (2005). Hyperspectral discrimination of tropical rain forest tree species at leaf to crown scales. Remote Sensing of Environment, 96(3):375-398.

[68] Close, D. C. and Beadle, C. L. (2003). The ecophysiology of foliar anthocyanin. The Botanical Review, 69(2):149-161.

[69] Cochrane, M. (2000). Using vegetation reflectance variability for species level classification of hyperspectral data. International Journal of Remote Sensing, 21(10):2075-2087.

[70] Cole-Rhodes, A., Johnson, K., Moigne, J. L., and Zavorin, I. (2003). Multiresolution registration of remote sensing imagery by optimization of mutual information using a stochastic gradient. Image Processing, IEEE Transactions on, 12(12):1495-1511.

[71] Colgan, M. S., Baldeck, C. A., Féret, J.-B., and Asner, G. P. (2012). Mapping savanna tree species at ecosystem scales using support vector machine classification and BRDF correction on airborne hyperspectral and LiDAR data. Remote Sensing, 4(11):3462-3480.

[72] Cox, P. M., Betts, R. A., Jones, C. D., Spall, S. A., and Totterdell, I. J. (2000). Acceleration of global warming due to carbon-cycle feedbacks in a coupled climate model. Nature, 408(6809):184-187.

[73] Dahlin, K. M., Asner, G. P., and Field, C. B. (2013). Environmental and community controls on plant canopy chemistry in a Mediterranean-type ecosystem. Proceedings of the National Academy of Sciences, 110(17):6895-6900.

[74] Dalponte, M., Bruzzone, L., and Gianelle, D. (2008). Fusion of hyperspectral and LiDAR remote sensing data for classification of complex forest areas. Geoscience and Remote Sensing, IEEE Transactions on, 46(5):1416-1427.

[75] Dalponte, M., Bruzzone, L., and Gianelle, D. (2011). A system for the estimation of single-tree stem diameter and volume using multireturn LiDAR data. Geoscience and Remote Sensing, IEEE Transactions on, 49(7):2479-2490.

[76] Dalponte, M., Bruzzone, L., and Gianelle, D. (2012). Tree species classification in the Southern Alps based on the fusion of very high geometrical resolution multispectral/hyperspectral images and LiDAR data. Remote Sensing of Environment, 123:258-270.

[77] Dalponte, M., Bruzzone, L., Vescovo, L., and Gianelle, D. (2009). The role of spectral resolution and classifier complexity in the analysis of hyperspectral images of forest areas. Remote Sensing of Environment, 113(11):2345-2355.

[78] Dalponte, M., Ørka, H. O., Ene, L. T., Gobakken, T., and Næsset, E. (2014). Tree crown delineation and tree species classification in boreal forests using hyperspectral and ALS data. Remote Sensing of Environment, 140:306-317.

[79] Dalponte, M., Orka, H. O., Gobakken, T., Gianelle, D., and Næsset, E. (2013). Tree species classification in boreal forests with hyperspectral data. Geoscience and Remote Sensing, IEEE Transactions on, 51(5):2632-2645.

[80] Dawn, S., Saxena, V., and Sharma, B. (2010). Remote sensing image registration techniques: a survey. In Image and Signal Processing, pages 103-112. Springer. 
[81] Demmig-Adams, B. and Adams, W. W. (1996). The role of xanthophyll cycle carotenoids in the protection of photosynthesis. Trends in Plant science, 1(1):21-26.

[82] Demmig-Adams, B. and Adams Iii, W. (1992). Photoprotection and other responses of plants to high light stress. Annual review of plant biology, 43(1):599-626.

[83] Dinuls, R., Erins, G., Lorencs, A., Mednieks, I., and Sinica-Sinavskis, J. (2012). Tree species identification in mixed baltic forest using LiDAR and multispectral data. Selected Topics in Applied Earth Observations and Remote Sensing, IEEE Journal of, 5(2):594603.

[84] Do, C. B. and Cormier, F. (1991). Effects of low nitrate and high sugar concentrations on anthocyanin content and composition of grape (Vitis vinifera L.) cell suspension. Plant Cell Reports, 9(9):500-504.

[85] Duan, Y. and Tai, X.-C. (2012). Domain decomposition methods with graph cuts algorithms for total variation minimization. Advances in Computational Mathematics, 36(2):175-199.

[86] Duncanson, L., Cook, B., Hurtt, G., and Dubayah, R. (2014). An efficient, multilayered crown delineation algorithm for mapping individual tree structure across multiple ecosystems. Remote Sensing of Environment, 154:378-386.

[87] Edson, C. and Wing, M. G. (2011). Airborne Light Detection and Ranging (LiDAR) for individual tree stem location, height, and biomass measurements. Remote Sensing, 3(11):2494-2528.

[88] Espírito-Santo, F. D., Gloor, M., Keller, M., Malhi, Y., Saatchi, S., Nelson, B., Junior, R. C. O., Pereira, C., Lloyd, J., Frolking, S., et al. (2014). Size and frequency of natural forest disturbances and the Amazon forest carbon balance. Nature communications, 5.

[89] Eysn, L., Hollaus, M., Lindberg, E., Berger, F., Monnet, J.-M., Dalponte, M., Kobal, M., Pellegrini, M., Lingua, E., Mongus, D., and Pfeifer, N. (2015). A benchmark of LiDAR-based single tree detection methods using heterogeneous forest data from the Alpine space. Forests, 6(5):1721-1747.

[90] Feilhauer, H., Asner, G. P., Martin, R. E., and Schmidtlein, S. (2010). Brightnessnormalized partial least squares regression for hyperspectral data. Journal of Quantitative Spectroscopy and Radiative Transfer, 111(12):1947-1957.

[91] Féret, J.-B. and Asner, G. P. (2011). Spectroscopic classification of tropical forest species using radiative transfer modeling. Remote Sensing of Environment, 115(9):24152422.

[92] Féret, J.-B. and Asner, G. P. (2012). Semi-supervised methods to identify individual crowns of lowland tropical canopy species using imaging spectroscopy and LiDAR. Remote Sensing, 4(8):2457-2476.

[93] Féret, J.-B. and Asner, G. P. (2013). Tree species discrimination in tropical forests using airborne imaging spectroscopy. Geoscience and Remote Sensing, IEEE Transactions on, 51(1):73-84. 
[94] Field, C. B., Randerson, J. T., and Malmström, C. M. (1995). Global net primary production: combining ecology and remote sensing. Remote Sensing of Environment, 51(1):74-88.

[95] Filella, I. and Peñuelas, J. (1999). Altitudinal differences in UV absorbance, UV reflectance and related morphological traits of Quercus ilex and Rhododendron ferrugineum in the Mediterranean region. Plant Ecology, 145(1):157-165.

[96] Filella, I., Porcar-Castell, A., Munné-Bosch, S., Bäck, J., Garbulsky, M., and Peñuelas, J. (2009). PRI assessment of long-term changes in carotenoids/chlorophyll ratio and short-term changes in de-epoxidation state of the xanthophyll cycle. International Journal of Remote Sensing, 30(17):4443-4455.

[97] Finlayson, G., Drew, M., and Lu, C. (2009). Entropy minimization for shadow removal. International Journal of Computer Vision, 85(1):35-57.

[98] Fischer, B. and Modersitzki, J. (2003). Curvature based image registration. Journal of Mathematical Imaging and Vision, 18(1):81-85.

[99] Fischer, B. and Modersitzki, J. (2004). A unified approach to fast image registration and a new curvature based registration technique. Linear Algebra and its applications, 380:107-124.

[100] Fischler, M. and Bolles, R. (1981). Random sample consensus: a paradigm for model fitting with applications to image analysis and automated cartography. Communications of the ACM, 24(6):381-395.

[101] Fonseca, L. M. and Manjunath, B. (1996). Registration techniques for multisensor remotely sensed imagery. PE \& RS-Photogrammetric Engineering \& Remote Sensing, 62(9):1049-1056.

[102] Gamon, J., Penuelas, J., and Field, C. (1992). A narrow-waveband spectral index that tracks diurnal changes in photosynthetic efficiency. Remote Sensing of Environment, 41(1):35-44.

[103] Gamon, J., Serrano, L., and Surfus, J. (1997). The photochemical reflectance index: an optical indicator of photosynthetic radiation use efficiency across species, functional types, and nutrient levels. Oecologia, 112(4):492-501.

[104] Gamon, J. and Surfus, J. (1999). Assessing leaf pigment content and activity with a reflectometer. New Phytologist, 143(1):105-117.

[105] Garbulsky, M. F., Peñuelas, J., Gamon, J., Inoue, Y., and Filella, I. (2011). The photochemical reflectance index (PRI) and the remote sensing of leaf, canopy and ecosystem radiation use efficiencies: a review and meta-analysis. Remote Sensing of Environment, 115(2):281-297.

[106] Garvin, J., Bufton, J., Blair, J., Harding, D., Luthcke, S., Frawley, J., and Rowlands, D. (1998). Observations of the Earth's topography from the Shuttle Laser Altimeter (SLA): laser-pulse echo-recovery measurements of terrestrial surfaces. Physics and Chemistry of the Earth, 23(9):1053-1068. 
[107] Gea-Izquierdo, G., Fonti, P., Cherubini, P., Martín-Benito, D., Chaar, H., and Cañellas, I. (2012). Xylem hydraulic adjustment and growth response of Quercus canariensis Willd. to climatic variability. Tree physiology, page tps026.

[108] Ghosh, A., Fassnacht, F. E., Joshi, P., and Koch, B. (2014). A framework for mapping tree species combining hyperspectral and LiDAR data: role of selected classifiers and sensor across three spatial scales. International Journal of Applied Earth Observation and Geoinformation, 26:49-63.

[109] Gitelson, A. A., Chivkunova, O. B., and Merzlyak, M. N. (2009). Nondestructive estimation of anthocyanins and chlorophylls in anthocyanic leaves. American Journal of Botany, 96(10):1861-1868.

[110] Gitelson, A. A., Keydan, G. P., and Merzlyak, M. N. (2006). Three-band model for noninvasive estimation of chlorophyll, carotenoids, and anthocyanin contents in higher plant leaves. Geophysical Research Letters, 33(11).

[111] Gitelson, A. A., Merzlyak, M. N., and Chivkunova, O. B. (2001). Optical properties and nondestructive estimation of anthocyanin content in plant leaves. Photochemistry and Photobiology, 74(1):38-45.

[112] Gitelson, A. A., Zur, Y., Chivkunova, O. B., and Merzlyak, M. N. (2002). Assessing carotenoid content in plant leaves with reflectance spectroscopy. Photochemistry and Photobiology, 75(3):272-281.

[113] Golding, N. and Betts, R. (2008). Fire risk in Amazonia due to climate change in the HadCM3 climate model: potential interactions with deforestation. Global Biogeochemical Cycles, 22(4).

[114] Goncalves, H., Corte-Real, L., Gonçalves, J., et al. (2011). Automatic image registration through image segmentation and SIFT. Geoscience and Remote Sensing, IEEE Transactions on, 49(7):2589-2600.

[115] Gonçalves, H., Gonçalves, J., Corte-Real, L., et al. (2011). HAIRIS: A method for automatic image registration through histogram-based image segmentation. Image Processing, IEEE Transactions on, 20(3):776-789.

[116] Goodwin, N., Turner, R., and Merton, R. (2005). Classifying Eucalyptus forests with high spatial and spectral resolution imagery: an investigation of individual species and vegetation communities. Australian Journal of Botany, 53(4):337-345.

[117] Group, I. T. C. W. et al. (1998). The terrestrial carbon cycle: implications for the Kyoto Protocol. Science, 280(5368):1393.

[118] Haber, E. and Modersitzki, J. (2006a). Intensity gradient based registration and fusion of multi-modal images. In Medical Image Computing and Computer-Assisted Intervention-MICCAI 2006, pages 726-733. Springer.

[119] Haber, E. and Modersitzki, J. (2006b). A multilevel method for image registration. SIAM Journal on Scientific Computing, 27(5):1594-1607. 
[120] Hadamard, J. (1902). Sur les problèmes aux dérivées partielles et leur signification physique. Princeton university bulletin, 13(49-52):28.

[121] Harding, D. J. and Carabajal, C. C. (2005). ICESat waveform measurements of withinfootprint topographic relief and vegetation vertical structure. Geophysical Research Letters, 32(21).

[122] Heinzel, J. and Koch, B. (2012). Investigating multiple data sources for tree species classification in temperate forest and use for single tree delineation. International Journal of Applied Earth Observation and Geoinformation, 18:101-110.

[123] Henn, S. (2006). A full curvature based algorithm for image registration. Journal of Mathematical Imaging and Vision, 24(2):195-208.

[124] Hesketh, M. and Sánchez-Azofeifa, G. A. (2012). The effect of seasonal spectral variation on species classification in the Panamanian tropical forest. Remote Sensing of Environment, 118:73-82.

[125] Hill, M., Held, A., Leuning, R., Coops, N., Hughes, D., and Cleugh, H. (2006). MODIS spectral signals at a flux tower site: relationships with high-resolution data, and $\mathrm{CO}_{2}$ flux and light use efficiency measurements. Remote Sensing of Environment, 103(3):351-368.

[126] Hill, R., Wilson, A., George, M., and Hinsley, S. (2010). Mapping tree species in temperate deciduous woodland using time-series multi-spectral data. Applied Vegetation Science, 13(1):86-99.

[127] Hoffbeck, J. P., Landgrebe, D., et al. (1994). Effect of radiance-to-reflectance transformation and atmosphere removal on maximum likelihood classification accuracy of high-dimensional remote sensing data. In Geoscience and Remote Sensing Symposium, 1994. IGARSS'94. Surface and Atmospheric Remote Sensing: Technologies, Data Analysis and Interpretation., International, volume 4, pages 2538-2540. IEEE.

[128] Höfle, B., Hollaus, M., and Hagenauer, J. (2012). Urban vegetation detection using radiometrically calibrated small-footprint full-waveform airborne LiDAR data. ISPRS Journal of Photogrammetry and Remote Sensing, 67:134-147.

[129] Hollas, J. M. (2004). Modern spectroscopy. John Wiley \& Sons.

[130] Holmgren, J., Persson, Å., and Söderman, U. (2008). Species identification of individual trees by combining high resolution LiDAR data with multi-spectral images. International Journal of Remote Sensing, 29(5):1537-1552.

[131] Hong, G. and Zhang, Y. (2008). Wavelet-based image registration technique for high-resolution remote sensing images. Computers \& Geosciences, 34(12):1708-1720.

[132] Houghton, R., Lawrence, K., Hackler, J., and Brown, S. (2001). The spatial distribution of forest biomass in the Brazilian Amazon: a comparison of estimates. Global Change Biology, 7(7):731-746. 
[133] Hu, H., Feng, J., Yu, C., and Zhou, J. (2013). Multi-class constrained normalized cut with hard, soft, unary and pairwise priors and its applications to object segmentation. Image Processing, IEEE Transactions on, 22(11):4328-4340.

[134] Huang, S., Crabtree, R., Potter, C., and Gross, P. (2009). Estimating the quantity and quality of coarse woody debris in Yellowstone post-fire forest ecosystem from fusion of SAR and optical data. Remote Sensing of Environment, 113(9):1926-1938.

[135] Hudak, A. and Wessman, C. (1998). Textural analysis of historical aerial photography to characterize woody plant encroachment in South African savanna. Remote Sensing of Environment, 66(3):317-330.

[136] Hudak, A. T., Crookston, N. L., Evans, J. S., Hall, D. E., and Falkowski, M. J. (2008). Nearest neighbor imputation of species-level, plot-scale forest structure attributes from LiDAR data. Remote Sensing of Environment, 112(5):2232-2245.

[137] Huete, A. R., Didan, K., Shimabukuro, Y. E., Ratana, P., Saleska, S. R., Hutyra, L. R., Yang, W., Nemani, R. R., and Myneni, R. (2006). Amazon rainforests green-up with sunlight in dry season. Geophysical Research Letters, 33(6).

[138] Hughes, G. P. (1968). On the mean accuracy of statistical pattern recognizers. Information Theory, IEEE Transactions on, 14(1):55-63.

[139] Hyyppä, J., Kelle, O., Lehikoinen, M., and Inkinen, M. (2001). A segmentation-based method to retrieve stem volume estimates from 3-D tree height models produced by laser scanners. Geoscience and Remote Sensing, IEEE Transactions on, 39(5):969-975.

[140] Immitzer, M., Atzberger, C., and Koukal, T. (2012). Tree species classification with random forest using very high spatial resolution 8-band WorldView-2 satellite data. Remote Sensing, 4(9):2661-2693.

[141] Inglada, J. and Giros, A. (2004). On the possibility of automatic multisensor image registration. Geoscience and Remote Sensing, IEEE Transactions on, 42(10):2104-2120.

[142] Jacquemoud, S., Verhoef, W., Baret, F., Bacour, C., Zarco-Tejada, P. J., Asner, G. P., François, C., and Ustin, S. L. (2009). PROSPECT+ SAIL models: a review of use for vegetation characterization. Remote Sensing of Environment, 113:S56-S66.

[143] Jakubowski, M. K., Li, W., Guo, Q., and Kelly, M. (2013). Delineating individual trees from LiDAR data: a comparison of vector-and raster-based segmentation approaches. Remote Sensing, 5(9):4163-4186.

[144] Jenkins, J. C., Chojnacky, D. C., Heath, L. S., and Birdsey, R. A. (2003). National-scale biomass estimators for United States tree species. Forest Science, 49(1):12-35.

[145] Jolliffe, I. (2002). Principal component analysis. Wiley Online Library.

[146] Jones, T. G., Coops, N. C., and Sharma, T. (2010). Assessing the utility of airborne hyperspectral and LiDAR data for species distribution mapping in the coastal Pacific Northwest, Canada. Remote Sensing of Environment, 114(12):2841-2852. 
[147] Jordán, A., Martínez-Zavala, L., and Bellinfante, N. (2008). Heterogeneity in soil hydrological response from different land cover types in southern Spain. Catena, 74(2):137143.

[148] Kaartinen, H., Hyyppä, J., Yu, X., Vastaranta, M., Hyyppä, H., Kukko, A., Holopainen, M., Heipke, C., Hirschmugl, M., Morsdorf, F., et al. (2012). An international comparison of individual tree detection and extraction using airborne laser scanning. Remote Sensing, 4(4):950-974.

[149] Kaasalainen, S., Hyyppa, H., Kukko, A., Litkey, P., Ahokas, E., Hyyppa, J., Lehner, H., Jaakkola, A., Suomalainen, J., Akujarvi, A., and etal (2009). Radiometric calibration of LiDAR intensity with commercially available reference targets. Geoscience and Remote Sensing, IEEE Transactions on, 47(2):588-598.

[150] Kim, T. and Im, Y. (2003). Automatic satellite image registration by combination of matching and random sample consensus. Geoscience and Remote Sensing, IEEE Transactions on, 41(5):1111-1117.

[151] Koch, B. (2010). Status and future of laser scanning, synthetic aperture radar and hyperspectral remote sensing data for forest biomass assessment. ISPRS Journal of Photogrammetry and Remote Sensing, 65(6):581-590.

[152] Koch, B., Heyder, U., and Weinacker, H. (2006). Detection of individual tree crowns in airborne LiDAR data. Photogrammetric Engineering and Remote Sensing, 72(4):357.

[153] Koetz, B., Morsdorf, F., Sun, G., Ranson, K., Itten, K., and Allgöwer, B. (2006). Inversion of a LiDAR waveform model for forest biophysical parameter estimation. Geoscience and Remote Sensing Letters, IEEE, 3(1):49-53.

[154] Körner, C. (2003). Alpine plant life: functional plant ecology of high mountain ecosystems; with 47 tables. Springer Science \& Business Media.

[155] Korpela, I., Ørka, H., Maltamo, M., Tokola, T., Hyyppä, J., and etal (2010a). Tree species classification using airborne LiDAR-effects of stand and tree parameters, downsizing of training set, intensity normalization, and sensor type. Silva Fennica, 44(2):319-339.

[156] Korpela, I., Ørka, H. O., Hyyppä, J., Heikkinen, V., and Tokola, T. (2010b). Range and AGC normalization in airborne discrete-return LiDAR intensity data for forest canopies. ISPRS Journal of Photogrammetry and Remote Sensing, 65(4):369-379.

[157] Kroon, D. and Slump, C. (2009). MRI modalitiy transformation in demon registration. In Biomedical Imaging: From Nano to Macro, 2009. ISBI'09. IEEE International Symposium on, pages 963-966. IEEE.

[158] Kurz, W. and Apps, M. (2006). Developing Canada's national forest carbon monitoring, accounting and reporting system to meet the reporting requirements of the Kyoto Protocol. Mitigation and Adaptation Strategies for Global Change, 11(1):33-43.

[159] Kwak, D.-A., Lee, W.-K., Lee, J.-H., Biging, G. S., and Gong, P. (2007). Detection of individual trees and estimation of tree height using LiDAR data. Journal of Forest Research, 12(6):425-434. 
[160] Kytridis, V.-P., Karageorgou, P., Levizou, E., and Manetas, Y. (2008). Intra-species variation in transient accumulation of leaf anthocyanins in Cistus creticus during winter: evidence that anthocyanins may compensate for an inherent photosynthetic and photoprotective inferiority of the red-leaf phenotype. Journal of Plant Physiology, 165(9):952-959.

[161] Latifi, H., Nothdurft, A., and Koch, B. (2010). Non-parametric prediction and mapping of standing timber volume and biomass in a temperate forest: application of multiple optical/LiDAR-derived predictors. Forestry, 83(4):395-407.

[162] Lawanson, A., Akindele, B., Fasalojo, P., and Akpe, B. (1972). Time-course of anthocyanin formation during deficiencies of nitrogen, phosphorus and potassium in seedlings of Zea mays Linn. var. ES 1. Zeitschrift für Pflanzenphysiologie, 66(3):251-253.

[163] Lawrence, R. L., Wood, S. D., and Sheley, R. L. (2006). Mapping invasive plants using hyperspectral imagery and Breiman Cutler classifications (RandomForest). Remote Sensing of Environment, 100(3):356-362.

[164] Le Moigne, J., Netanyahu, N. S., and Eastman, R. D. (2011). Image registration for remote sensing. Cambridge University Press.

[165] Le Toan, T., Beaudoin, A., Riom, J., and Guyon, D. (1992). Relating forest biomass to SAR data. Geoscience and Remote Sensing, IEEE Transactions on, 30(2):403-411.

[166] Lee, D. W., O’keefe, J., Holbrook, N. M., and Feild, T. S. (2003). Pigment dynamics and autumn leaf senescence in a New England deciduous forest, eastern USA. Ecological Research, 18(6):677-694.

[167] Lee, H., Slatton, K., Roth, B., and JR, W. C. (2010). Adaptive clustering of airborne LiDAR data to segment individual tree crowns in managed pine forests. International Journal of Remote Sensing, 31(1):117-139.

[168] Lee, J., Cai, X., Dalponte, M., Lellmann, J., Schönlieb, C.-B., and Coomes, D. (2015a). 3D Individual Tree Segmentation from Fully Integrated LiDAR, Hyperspectral Imagery and Aerial Photographs. Submitted for publication.

[169] Lee, J., Cai, X., Dalponte, M., Lellmann, J., Schönlieb, C.-B., and Coomes, D. (2015b). Individual tree species classification from airborne multi-sensor imagery. Submitted for publication.

[170] Lee, J., Cai, X., Schönlieb, C.-B., and Coomes, D. (2015c). Mapping individual trees from airborne multi-sensor imagery. In Geoscience and Remote Sensing Symposium (IGARSS), 2015 IEEE International, pages 5411-5414. IEEE.

[171] Lee, J., Cai, X., Schönlieb, C.-B., and Coomes, D. (2015d). Nonparametric Image Registration of Airborne LiDAR, Hyperspectral and Photographic Imagery of Wooded Landscapes. Geoscience and Remote Sensing, IEEE Transactions on, 53(11):6073-6084.

[172] Lefsky, M., Cohen, W., and Spies, T. (2001). An evaluation of alternate remote sensing products for forest inventory, monitoring, and mapping of Douglas-fir forests in western Oregon. Canadian Journal of Forest Research, 31(1):78-87. 
[173] Lefsky, M. A. (2010). A global forest canopy height map from the Moderate Resolution Imaging Spectroradiometer and the Geoscience Laser Altimeter System. Geophysical Research Letters, 37(15).

[174] Lefsky, M. A., Cohen, W. B., Parker, G. G., and Harding, D. J. (2002). LiDAR remote sensing for ecosystem studies. BioScience, 52(1):19-30.

[175] Lefsky, M. A., Harding, D. J., Keller, M., Cohen, W. B., Carabajal, C. C., Del Bom Espirito-Santo, F., Hunter, M. O., and de Oliveira, R. (2005). Estimates of forest canopy height and aboveground biomass using ICESat. Geophysical Research Letters, 32(22).

[176] Leow, A. D., Yanovsky, I., Chiang, M.-C., Lee, A. D., Klunder, A. D., Lu, A., Becker, J. T., Davis, S. W., Toga, A. W., and Thompson, P. M. (2007). Statistical properties of Jacobian maps and the realization of unbiased large-deformation nonlinear image registration. Medical Imaging, IEEE Transactions on, 26(6):822-832.

[177] Li, H., Manjunath, B., and Mitra, S. (1995). A contour-based approach to multisensor image registration. Image Processing, IEEE Transactions on, 4(3):320-334.

[178] Li, Q., Wang, G., Liu, J., and Chen, S. (2009). Robust scale-invariant feature matching for remote sensing image registration. Geoscience and Remote Sensing Letters, IEEE, 6(2):287-291.

[179] Li, W., Guo, Q., Jakubowski, M. K., and Kelly, M. (2012). A new method for segmenting individual trees from the LiDAR point cloud. Photogrammetric Engineering \& Remote Sensing, 78(1):75-84.

[180] Liang, J., Liu, X., Huang, K., Li, X., Wang, D., and Wang, X. (2014). Automatic Registration of Multisensor Images Using an Integrated Spatial and Mutual Information (SMI) Metric. Geoscience and Remote Sensing, IEEE Transactions on, 51(1):603-615.

[181] Lim, K., Treitz, P., Wulder, M., St-Onge, B., and Flood, M. (2003). LiDAR remote sensing of forest structure. Progress in Physical Geography, 27(1):88-106.

[182] Lowe, D. G. (2004). Distinctive image features from scale-invariant keypoints. International Journal of Computer Vision, 60(2):91-110.

[183] Löwe, H., Seufert, G., and Raes, F. (2000). Comparison of methods used within Member States for estimating CO2 emissions and sinks according to UNFCCC and EU Monitoring Mechanism: forest and other wooded land. Biotechnologie, Agronomie, Société et Environnement, 4(4):315-319.

[184] Luyssaert, S., Schulze, E.-D., Börner, A., Knohl, A., Hessenmöller, D., Law, B. E., Ciais, P., and Grace, J. (2008). Old-growth forests as global carbon sinks. Nature, 455(7210):213-215.

[185] Magnussen, S. and Boudewyn, P. (1998). Derivations of stand heights from airborne laser scanner data with canopy-based quantile estimators. Canadian journal of forest research, 28(7):1016-1031. 
[186] Malhi, Y., Aragão, L. E., Galbraith, D., Huntingford, C., Fisher, R., Zelazowski, P., Sitch, S., McSweeney, C., and Meir, P. (2009). Exploring the likelihood and mechanism of a climate-change-induced dieback of the Amazon rainforest. Proceedings of the National Academy of Sciences, 106(49):20610-20615.

[187] Maltamo, M., Eerikäinen, K., Packalén, P., and Hyyppä, J. (2006a). Estimation of stem volume using laser scanning-based canopy height metrics. Forestry, 79(2):217-229.

[188] Maltamo, M., Malinen, J., Packalén, P., Suvanto, A., and Kangas, J. (2006b). Nonparametric estimation of stem volume using airborne laser scanning, aerial photography, and stand-register data. Canadian Journal of Forest Research, 36(2):426-436.

[189] Maltamo, M., Næsset, E., and Vauhkonen, J. (2014). Forestry applications of airborne laser scanning. Springer.

[190] Maniatis, D. and Mollicone, D. (2010). Options for sampling and stratification for national forest inventories to implement REDD+ under the UNFCCC. Carbon balance and management, 5(1):1-14.

[191] McRoberts, R. E. and Tomppo, E. O. (2007). Remote sensing support for national forest inventories. Remote Sensing of Environment, 110(4):412-419.

[192] Means, J. E., Acker, S. A., Fitt, B. J., Renslow, M., Emerson, L., Hendrix, C. J., et al. (2000). Predicting forest stand characteristics with airborne scanning LiDAR. Photogrammetric Engineering and Remote Sensing, 66(11):1367-1372.

[193] Modersitzki, J. (2003). Numerical methods for image registration. OUP Oxford.

[194] Modersitzki, J. (2009). FAIR: flexible algorithms for image registration, volume 6. SIAM.

[195] Moreau, S. and Le Toan, T. (2003). Biomass quantification of Andean wetland forages using ERS satellite SAR data for optimizing livestock management. Remote Sensing of Environment, 84(4):477-492.

[196] Morel, A. C., Saatchi, S. S., Malhi, Y., Berry, N. J., Banin, L., Burslem, D., Nilus, R., and Ong, R. C. (2011). Estimating aboveground biomass in forest and oil palm plantation in Sabah, Malaysian Borneo using ALOS PALSAR data. Forest Ecology and Management, 262(9):1786-1798.

[197] Morsdorf, F., Meier, E., Kötz, B., Itten, K. I., Dobbertin, M., and Allgöwer, B. (2004). LiDAR-based geometric reconstruction of boreal type forest stands at single tree level for forest and wildland fire management. Remote Sensing of Environment, 92(3):353-362.

[198] Morton, D. C., Nagol, J., Carabajal, C. C., Rosette, J., Palace, M., Cook, B. D., Vermote, E. F., Harding, D. J., and North, P. R. (2014). Amazon forests maintain consistent canopy structure and greenness during the dry season. Nature, 506(7487):221-224.

[199] Myneni, R. B., Ramakrishna, R., Nemani, R., and Running, S. W. (1997). Estimation of global leaf area index and absorbed PAR using radiative transfer models. Geoscience and Remote Sensing, IEEE Transactions on, 35(6):1380-1393. 
[200] Naidoo, L., Cho, M., Mathieu, R., and Asner, G. (2012). Classification of savanna tree species, in the Greater Kruger National Park region, by integrating hyperspectral and LiDAR data in a Random Forest data mining environment. ISPRS Journal of Photogrammetry and Remote Sensing, 69:167-179.

[201] Nakashizuka, T., Katsuki, T., and Tanaka, H. (1995). Forest canopy structure analyzed by using aerial photographs. Ecological Research, 10(1):13-18.

[202] Nelson, R. (2013). How did we get here? An early history of forestry lidar1. Canadian Journal of Remote Sensing, 39(sup1):S6-S17.

[203] NERC-DAN (2011). Natural environment research council airborne research and survey facility data analysis node report.

[204] NEWFOR, P. (2012). Alpine Space Programme, European Territorial Cooperation 2007-2013-Project NEWFOR.

[205] Ørka, H. O., Næsset, E., and Bollandsås, O. M. (2009). Classifying species of individual trees by intensity and structure features derived from airborne laser scanner data. Remote Sensing of Environment, 113(6):1163-1174.

[206] Packalén, P. and Maltamo, M. (2007). The k-MSN method for the prediction of species-specific stand attributes using airborne laser scanning and aerial photographs. Remote Sensing of Environment, 109(3):328-341.

[207] Palenichka, R., Doyon, F., Lakhssassi, A., and Zaremba, M. B. (2013). Multi-scale segmentation of forest areas and tree detection in LiDAR images by the attentive vision method. Selected Topics in Applied Earth Observations and Remote Sensing, IEEE Journal of, 6(3):1313-1323.

[208] Paloscia, S., Macelloni, G., Pampaloni, P., and Sigismondi, S. (1999). The potential of C-and L-band SAR in estimating vegetation biomass: the ERS-1 and JERS-1 experiments. Geoscience and Remote Sensing, IEEE Transactions on, 37(4):2107-2110.

[209] Parmehr, E., Fraser, C., Zhang, C., and Leach, J. (2014). Automatic registration of optical imagery with 3D LiDAR data using statistical similarity. ISPRS Journal of Photogrammetry and Remote Sensing, 88:28-40.

[210] Parmesan, C. and Yohe, G. (2003). A globally coherent fingerprint of climate change impacts across natural systems. Nature, 421(6918):37-42.

[211] Patenaude, G., Hill, R., Milne, R., Gaveau, D. L., Briggs, B., and Dawson, T. (2004). Quantifying forest above ground carbon content using LiDAR remote sensing. Remote Sensing of Environment, 93(3):368-380.

[212] Patenaude, G., Milne, R., and Dawson, T. P. (2005). Synthesis of remote sensing approaches for forest carbon estimation: reporting to the Kyoto Protocol. Environmental Science \& Policy, 8(2):161-178.

[213] Pearlman, J. S., Barry, P. S., Segal, C. C., Shepanski, J., Beiso, D., and Carman, S. L. (2003). Hyperion, a space-based imaging spectrometer. Geoscience and Remote Sensing, IEEE Transactions on, 41(6):1160-1173. 
[214] Pearson, R. G. and Dawson, T. P. (2003). Predicting the impacts of climate change on the distribution of species: are bioclimate envelope models useful? Global ecology and biogeography, 12(5):361-371.

[215] Penuelas, J., Filella, I., Biel, C., Serrano, L., and Save, R. (1993). The reflectance at the 950-970 nm region as an indicator of plant water status. International journal of remote sensing, 14(10):1887-1905.

[216] Peñuelas, J., Pinol, J., Ogaya, R., and Filella, I. (1997). Estimation of plant water concentration by the reflectance water index WI (R900/R970). International Journal of Remote Sensing, 18(13):2869-2875.

[217] Persson, A., Holmgren, J., and Söderman, U. (2002). Detecting and measuring individual trees using an airborne laser scanner. Photogrammetric Engineering and Remote Sensing, 68(9):925-932.

[218] Phillips, O. L., Aragão, L. E., Lewis, S. L., Fisher, J. B., Lloyd, J., López-González, G., Malhi, Y., Monteagudo, A., Peacock, J., Quesada, C. A., et al. (2009). Drought sensitivity of the Amazon rainforest. Science, 323(5919):1344-1347.

[219] Pietrini, F., Iannelli, M., and Massacci, A. (2002). Anthocyanin accumulation in the illuminated surface of maize leaves enhances protection from photo-inhibitory risks at low temperature, without further limitation to photosynthesis. Plant, Cell \& Environment, 25(10):1251-1259.

[220] Popescu, S. C. (2007). Estimating biomass of individual pine trees using airborne LiDAR. Biomass and Bioenergy, 31(9):646-655.

[221] Popescu, S. C., Wynne, R. H., and Nelson, R. F. (2003). Measuring individual tree crown diameter with LiDAR and assessing its influence on estimating forest volume and biomass. Canadian journal of remote sensing, 29(5):564-577.

[222] Poulos, H. M., Berlyn, G. P., and Mills, S. A. (2012). Differential stress tolerance of four pines (Pinaceae) across the elevation gradient of the San Bernardino Mountains, Southern California, USA 1. The Journal of the Torrey Botanical Society, 139(1):96-108.

[223] Puttonen, E., Litkey, P., and Hyyppä, J. (2009). Individual tree species classification by illuminated-Shaded area separation. Remote Sensing, 2(1):19-35.

[224] Reitberger, J., Schnörr, C., Krzystek, P., and Stilla, U. (2009). 3D segmentation of single trees exploiting full waveform LiDAR data. ISPRS Journal of Photogrammetry and Remote Sensing, 64(6):561-574.

[225] Rempel, R. C. and Parker, A. K. (1964). An information note on an airborne laser terrain profiler for micro-relief studies. In Proceedings of the $3 \mathrm{rd}$ symposium on remote sensing of environment, University of Michigan Institute of, Science and Technology, pages 321-337.

[226] Richardson, A. D. and Berlyn, G. P. (2002). Spectral reflectance and photosynthetic properties of Betula papyrifera (Betulaceae) leaves along an elevational gradient on Mt. Mansfield, Vermont, USA. American Journal of Botany, 89(1):88-94. 
[227] Richardson, A. D., Berlyn, G. P., and Gregoire, T. G. (2001). Spectral reflectance of Picea rubens (Pinaceae) and Abies balsamea (Pinaceae) needles along an elevational gradient, Mt. Moosilauke, New Hampshire, USA. American Journal of Botany, 88(4):667676.

[Richter and Schläpfer] Richter, R. and Schläpfer, D. Atmospheric/topographic correction for airborne imagery.

[229] Rodríguez-Pérez, J. R., Riaño, D., Carlisle, E., Ustin, S., and Smart, D. R. (2007). Evaluation of hyperspectral reflectance indexes to detect grapevine water status in vineyards. American Journal of Enology and Viticulture, 58(3):302-317.

[230] Ross, J. (2012). The radiation regime and architecture of plant stands, volume 3. Springer Science \& Business Media.

[231] Rouse Jr, J., Haas, R., Schell, J., and Deering, D. (1974). Monitoring vegetation systems in the Great Plains with ERTS. NASA special publication, 351:309.

[232] Roy, D. P., Wulder, M., Loveland, T., Woodcock, C., Allen, R., Anderson, M., Helder, D., Irons, J., Johnson, D., Kennedy, R., et al. (2014). Landsat-8: Science and product vision for terrestrial global change research. Remote Sensing of Environment, 145:154172.

[233] Ruiz García, A., Herrera Grao, A. F., and Ferreras-Romero, M. (2006). Distribution of Trichoptera communities in the Hozgargantacatchment (Los Alcornocales Natural Park, SW Spain). International Review of Hydrobiology, 91(1):71-85.

[234] Saleska, S. R., Didan, K., Huete, A. R., and Da Rocha, H. R. (2007). Amazon forests green-up during 2005 drought. Science, 318(5850):612-612.

[235] Samanta, A., Ganguly, S., Hashimoto, H., Devadiga, S., Vermote, E., Knyazikhin, Y., Nemani, R. R., and Myneni, R. B. (2010). Amazon forests did not green-up during the 2005 drought. Geophysical Research Letters, 37(5).

[236] Secord, J. and Zakhor, A. (2007). Tree detection in urban regions using aerial LiDAR and image data. Geoscience and Remote Sensing Letters, IEEE, 4(2):196-200.

[237] Shi, J. and Malik, J. (2000). Normalized cuts and image segmentation. Pattern Analysis and Machine Intelligence, IEEE Transactions on, 22(8):888-905.

[238] Simard, M., Pinto, N., Fisher, J. B., and Baccini, A. (2011). Mapping forest canopy height globally with spaceborne LiDAR. Journal of Geophysical Research: Biogeosciences (2005-2012), 116(G4).

[239] Sims, D. A. and Gamon, J. A. (2002). Relationships between leaf pigment content and spectral reflectance across a wide range of species, leaf structures and developmental stages. Remote Sensing of Environment, 81(2):337-354.

[240] Solberg, S., Naesset, E., and Bollandsas, O. M. (2006). Single tree segmentation using airborne laser scanner data in a structurally heterogeneous spruce forest. Photogrammetric Engineering and Remote Sensing, 72(12):1369. 
[241] Somers, B. and Asner, G. P. (2012). Hyperspectral time series analysis of native and invasive species in Hawaiian rainforests. Remote Sensing, 4(9):2510-2529.

[242] Song, Z. L. and Zhang, J. (2010). Remote sensing image registration based on retrofitted SURF algorithm and trajectories generated from Lissajous figures. Geoscience and Remote Sensing Letters, IEEE, 7(3):491-495.

[243] Sotiras, A., Davatzikos, C., and Paragios, N. (2013). Deformable medical image registration: a survey. Medical Imaging, IEEE Transactions on, 32(7):1153-1190.

[244] Stewart, A., Chapman, W., Jenkins, G., Graham, I., Martin, T., and Crozier, A. (2001). The effect of nitrogen and phosphorus deficiency on flavonol accumulation in plant tissues. Plant, Cell \& Environment, 24(11):1189-1197.

[245] Suárez, J. C., Ontiveros, C., Smith, S., and Snape, S. (2005). Use of airborne LiDAR and aerial photography in the estimation of individual tree heights in forestry. Computers \& Geosciences, 31(2):253-262.

[246] Suárez, L., Zarco-Tejada, P. J., Sepulcre-Cantó, G., Pérez-Priego, O., Miller, J., Jiménez-Muñoz, J., and Sobrino, J. (2008). Assessing canopy PRI for water stress detection with diurnal airborne imagery. Remote Sensing of Environment, 112(2):560575.

[247] Suri, S. and Reinartz, P. (2010). Mutual-information-based registration of TerraSAR-X and Ikonos imagery in urban areas. Geoscience and Remote Sensing, IEEE Transactions on, 48(2):939-949.

[248] Taiz, L. and Zeiger, E. (2010). Plant physiology 5th Ed. Sunderland: Sinauer Assoc.

[249] Thuiller, W., Lavorel, S., Araújo, M. B., Sykes, M. T., and Prentice, I. C. (2005). Climate change threats to plant diversity in Europe. Proceedings of the National Academy of Sciences, 102(23):8245-8250.

[250] Tochon, G., Féret, J., Valero, S., Martin, R., Knapp, D., Salembier, P., Chanussot, J., and Asner, G. (2015). On the use of binary partition trees for the tree crown segmentation of tropical rainforest hyperspectral images. Remote Sensing of Environment, 159:318-331.

[251] Tomppo, E. (1993). Multi-source national forest inventory of Finland. International Archives of Photogrammetry and Remote Sensing, 29:671-671.

[252] Urbieta, I. R., Zavala, M. A., and Maranón, T. (2008). Human and non-human determinants of forest composition in southern Spain: evidence of shifts towards cork oak dominance as a result of management over the past century. Journal of Biogeography, 35(9):1688-1700.

[253] Ustin, S. L., Roberts, D. A., Gamon, J. A., Asner, G. P., and Green, R. O. (2004). Using imaging spectroscopy to study ecosystem processes and properties. BioScience, 54(6):523-534.

[254] Vercauteren, T., Pennec, X., Perchant, A., and Ayache, N. (2009). Diffeomorphic demons: efficient non-parametric image registration. NeuroImage, 45(1):S61-S72. 
[255] Vitousek, P. M., Field, C. B., and Matson, P. A. (1990). Variation in foliar $\delta^{13}$ C in Hawaiian Metrosideros polymorpha: a case of internal resistance? Oecologia, 84(3):362370 .

[256] Von Luxburg, U. (2007). A tutorial on spectral clustering. Statistics and computing, 17(4):395-416.

[257] Voss, M. and Sugumaran, R. (2008). Seasonal effect on tree species classification in an urban environment using hyperspectral data, LiDAR, and an object-oriented approach. Sensors, 8(5):3020-3036.

[258] Wahed, M., El-tawel, G., and El-karim, A. (2013). Automatic image registration technique of remote sensing images. International Journal of Advanced Computer Science \& Applications, 4(2).

[259] Wanner, W., Li, X., and Strahler, A. (1995). On the derivation of kernels for kerneldriven models of bidirectional reflectance. Journal of Geophysical Research: Atmospheres (1984-2012), 100(D10):21077-21089.

[260] Weng, J.-H., Liao, T.-S., Hwang, M.-Y., Chung, C.-C., Lin, C.-P., and Chu, C.-H. (2006). Seasonal variation in photosystem II efficiency and photochemical reflectance index of evergreen trees and perennial grasses growing at low and high elevations in subtropical Taiwan. Tree Physiology, 26(8):1097-1104.

[261] Wessels, K., Prince, S., Frost, P., and Van Zyl, D. (2004). Assessing the effects of human-induced land degradation in the former homelands of northern South Africa with a $1 \mathrm{~km}$ AVHRR NDVI time-series. Remote Sensing of Environment, 91(1):47-67.

[262] Wong, A., Clausi, D., et al. (2007). ARRSI: automatic registration of remote-sensing images. Geoscience and Remote Sensing, IEEE Transactions on, 45(5):1483-1493.

[263] Yang, Y. and Gao, X. (2009). Remote sensing image registration via active contour model. AEU-international journal of electronics and communications, 63(4):227-234.

[264] Yao, W., Krzystek, P., and Heurich, M. (2012). Tree species classification and estimation of stem volume and DBH based on single tree extraction by exploiting airborne full-waveform LiDAR data. Remote Sensing of Environment, 123:368-380.

[265] Ye, Y. and Shan, J. (2014). A local descriptor based registration method for multispectral remote sensing images with non-linear intensity differences. ISPRS Journal of Photogrammetry and Remote Sensing, 90:83-95.

[266] Yu, X., Hyyppä, J., Vastaranta, M., Holopainen, M., and Viitala, R. (2011). Predicting individual tree attributes from airborne laser point clouds based on the random forests technique. ISPRS Journal of Photogrammetry and remote sensing, 66(1):28-37.

[267] Yuan, X. and Yang, J. (2009). Sparse and low-rank matrix decomposition via alternating direction methods. preprint.

[268] Zhang, C. and Qiu, F. (2012). Mapping individual tree species in an urban forest using airborne LiDAR data and hyperspectral imagery. Photogrammetric Engineering \& Remote Sensing, 78(10):1079-1087. 
[269] Zhang, J., Rivard, B., Sánchez-Azofeifa, A., and Castro-Esau, K. (2006). Intra-and inter-class spectral variability of tropical tree species at La Selva, Costa Rica: implications for species identification using HYDICE imagery. Remote Sensing of Environment, 105(2):129-141.

[270] Zhao, K., Popescu, S., Meng, X., Pang, Y., and Agca, M. (2011). Characterizing forest canopy structure with LiDAR composite metrics and machine learning. Remote Sensing of Environment, 115(8):1978-1996.

[271] Zitova, B. and Flusser, J. (2003). Image registration methods: a survey. Image and vision computing, 21(11):977-1000.

[272] Zolkos, S., Goetz, S., and Dubayah, R. (2013). A meta-analysis of terrestrial aboveground biomass estimation using LiDAR remote sensing. Remote Sensing of Environment, 128:289-298. 
